\title{
Quantitative phosphoproteomics for studying B-cell receptor signaling in Burkitt's lymphoma
}

\author{
Dissertation \\ for the award of the degree \\ "Doctor rerum naturalium" (Dr. rer. nat.) \\ Doctoral program Biomolecules: Structure - Function - Dynamics \\ of the Georg-August-Universität Göttingen, \\ Faculty of Biology and Psychology
}

\author{
submitted by \\ Jasmin Corso \\ from Erlenbach am Main, Germany
}

Göttingen 2015 



\section{Members of the Thesis Committee:}

Prof. Dr. Henning Urlaub (Reviewer)

Prof. Dr. Reinhard Jahn (Reviewer)

Prof. Dr. Bernhard Küster
Bioanalytical Mass Spectrometry Group, Max Planck Institute for Biophysical Chemistry, Göttingen

Bioanalytics, Institute for Clinical Chemistry, University Medical Center Göttingen

Department of Neurobiology, Max Planck Institute for Biophysical Chemistry, Göttingen

Chair of Proteomics and Bioanalytics, Technische Universität München

Members of the Examination Board:

Prof. Dr. Steven Johnsen Department for General, Visceral and Pediatric Surgery, University Medical Center Göttingen

Prof. Dr. Matthias

Dobbelstein

PD Dr. Halyna Shcherbata
Institute of Molecular Oncology, University Medical Center Göttingen

Gene expression and signaling, Max Planck Institute for Biophysical Chemistry, Göttingen

Date of the oral examination: 18.02.2016 



\section{Affidavit}

Hereby, I declare that the presented thesis entitled 'Quantitative phosphoproteomics for studying B-cell receptor signaling in Burkitt's lymphoma' was written entirely by myself and that I have only used the sources and materials cited.

Göttingen, 22.12.2015

Jasmin Corso 



\section{Content}

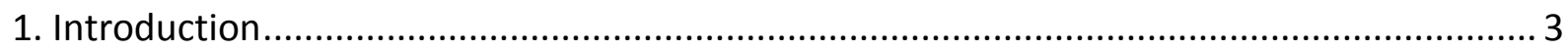

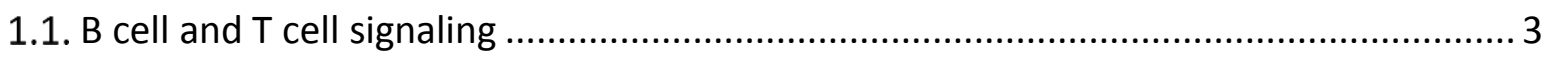

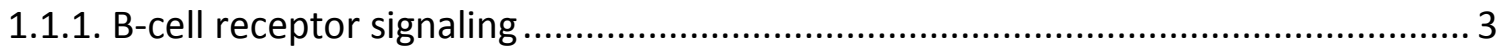

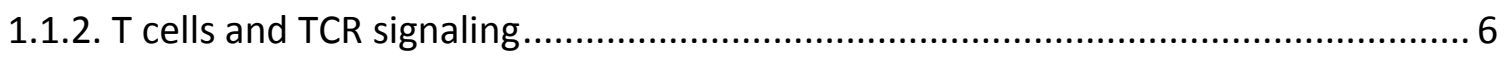

1.2. Hematologic malignancies - leukemia and lymphoma …........................................... 7

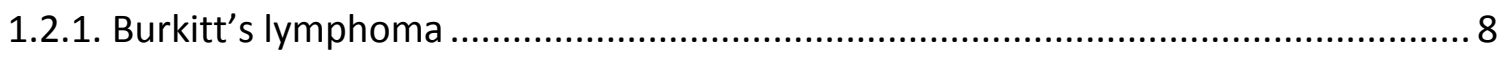

1.2.2. Acute myeloid leukemia ................................................................................ 9

1.3. Mass spectrometry-based proteome and PTM analysis ........................................... 11

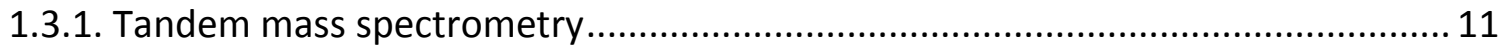

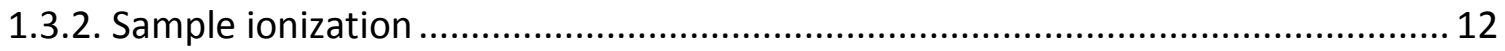

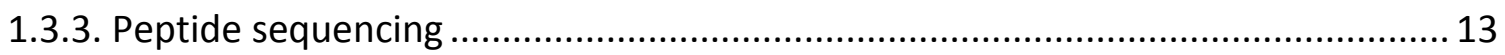

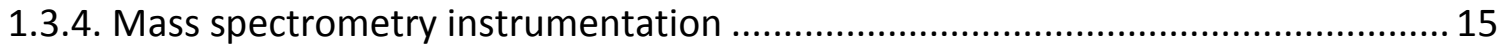

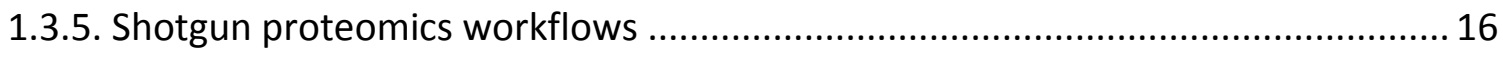

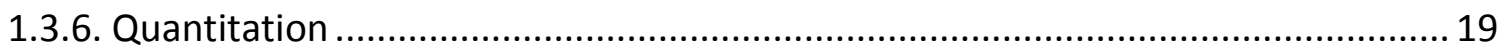

1.3.6.1. Stable isotope labeled amino acids in cell culture (SILAC) ............................. 20

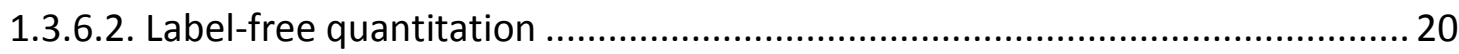

1.3.7. Peptide and protein identification .................................................................. 21

1.3.8. Identification of post-translational modification by mass spectrometry .............. 23

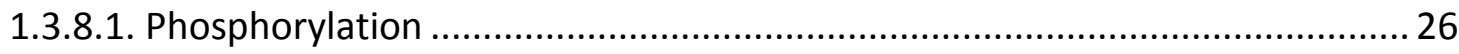

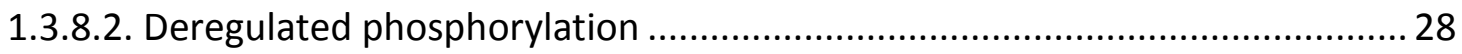

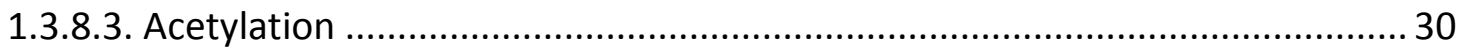

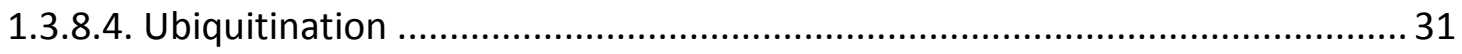

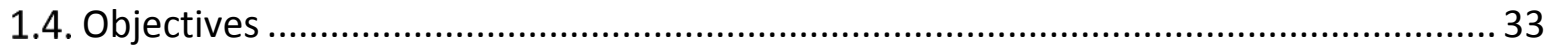

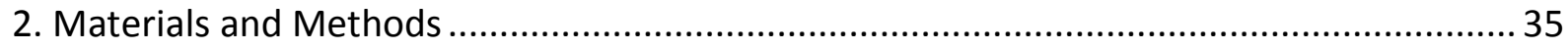

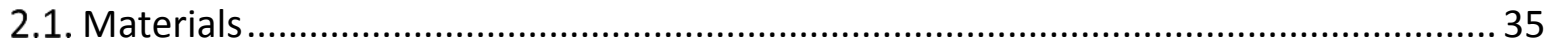

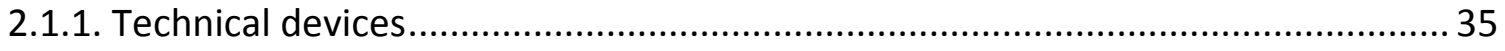

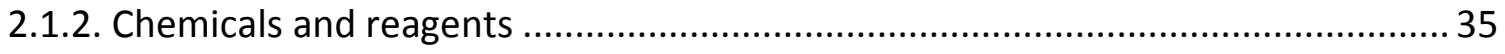

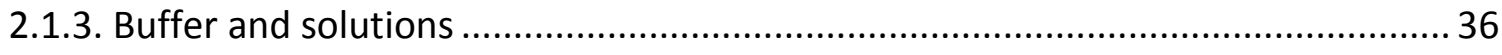

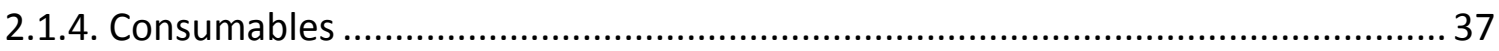

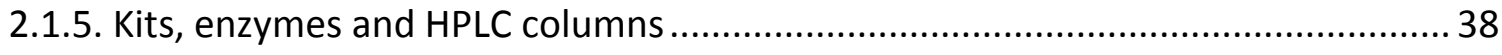

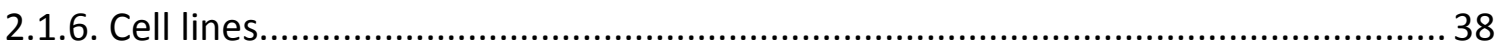

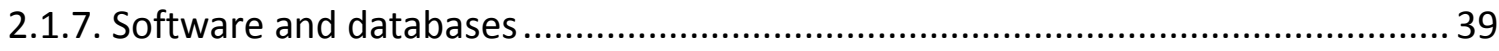

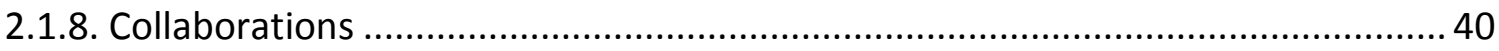




\section{Content}

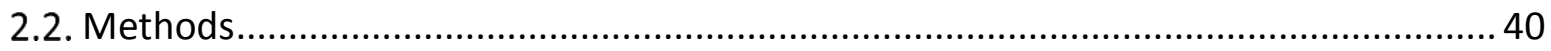

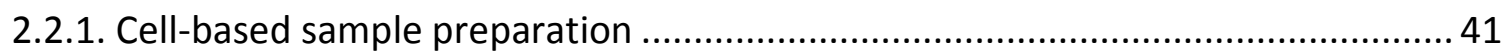

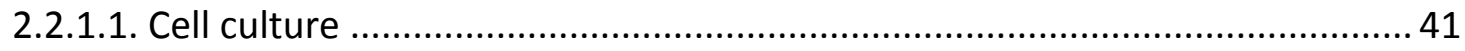

2.2.1.1.1. Burkitt's lymphoma and acute myeloid leukemia ................................ 41

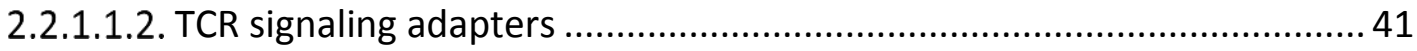

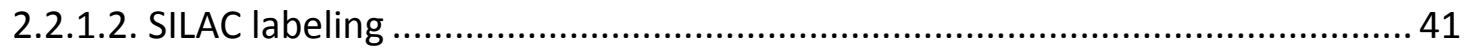

2.2.1.2.1. Burkitt's lymphoma and acute myeloid leukemia .................................. 41

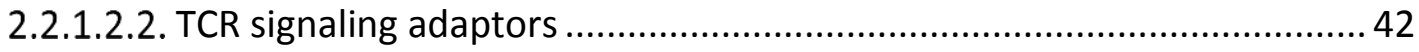

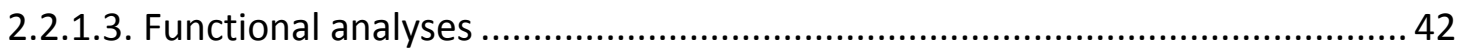

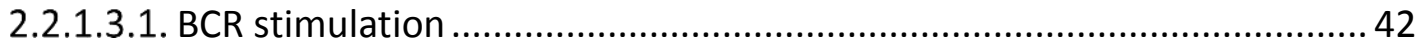

2.2.1.3.2. BCR knockdown and Syk inhibition ................................................... 42

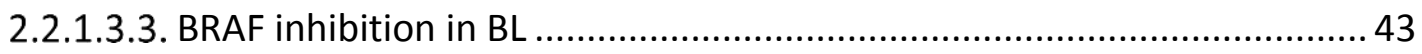

2.2.1.3.4. BRAF interactome analysis in $\mathrm{BL}$....................................................... 43

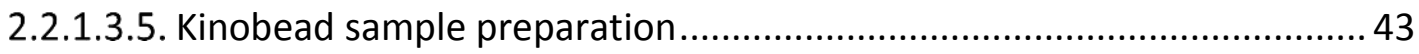

2.2.1.3.6. BTK interactome and inhibition in AML ............................................. 44

2.2.1.3.7. TCR stimulation and SLP76 affinity purification ..................................... 44

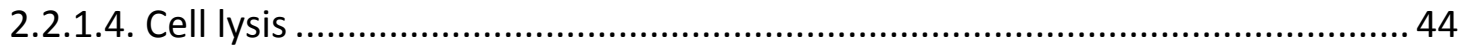

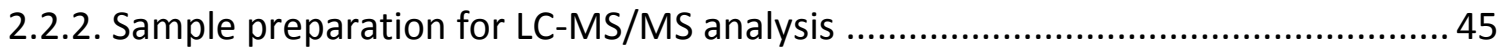

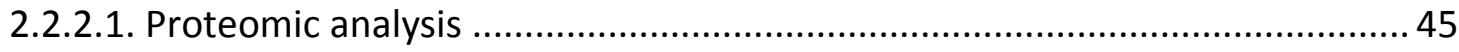

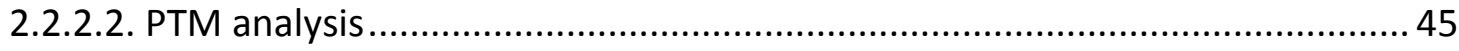

2.2.2.2.1. Global phosphoproteomic analysis........................................................ 46

2.2.2.2.2. Antibody-based PTM enrichment ......................................................... 47

2.2.2.2.3. Phosphoproteomic analysis of TCR signaling adapters .......................... 48

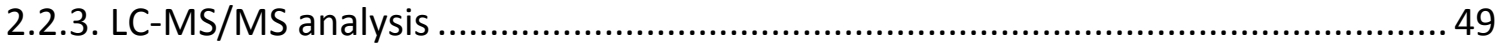

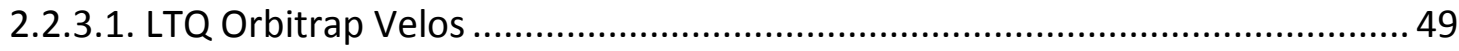

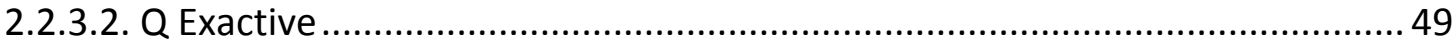

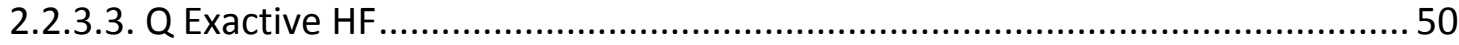

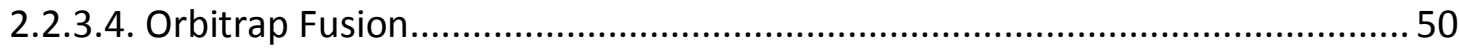

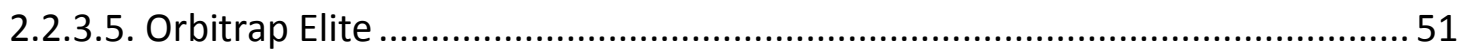

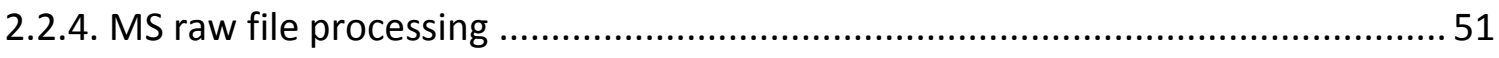

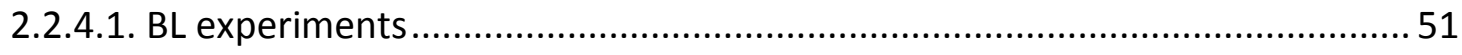

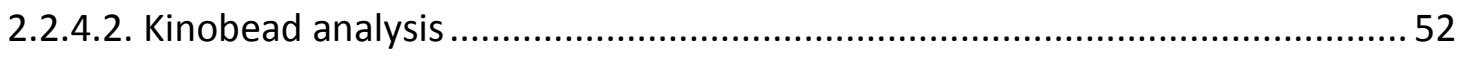

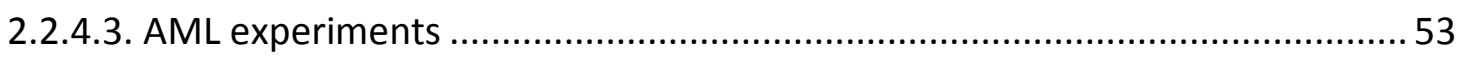

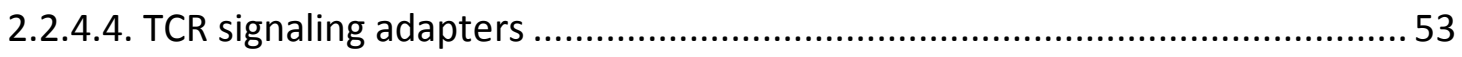

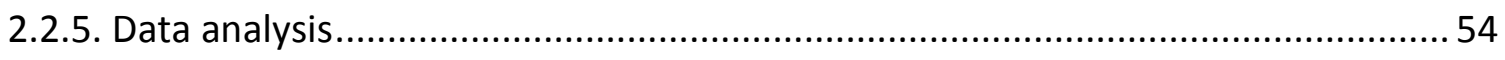

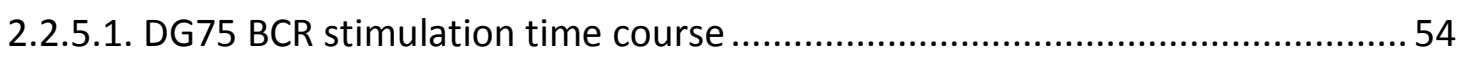




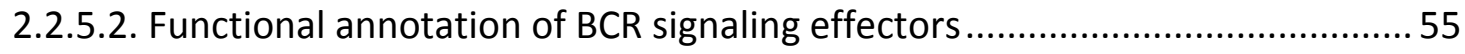

2.2.5.3. BCR stimulation time course in three distinct BL cell lines ............................ 55

2.2.5.4. Antigen-dependent and -independent signaling in DG75 ............................ 56

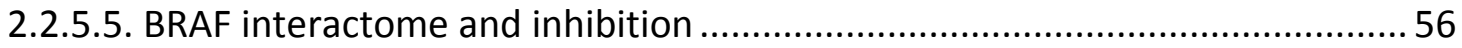

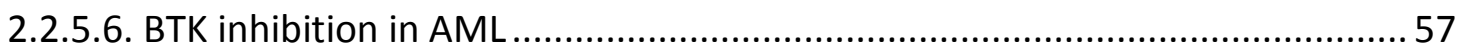

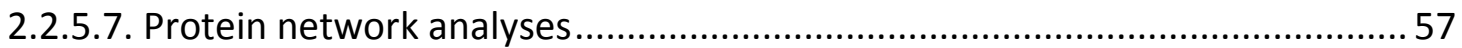

2.2.5.7.1. Phosphorylation interaction network analysis in BL.............................. 57

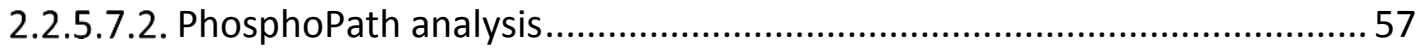

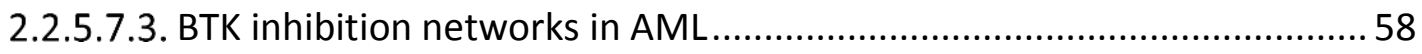

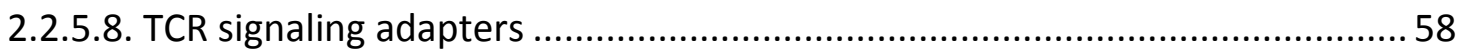

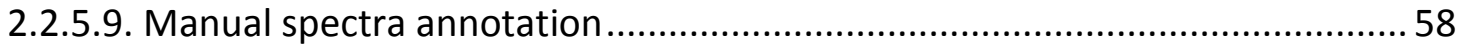

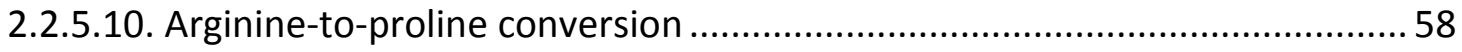

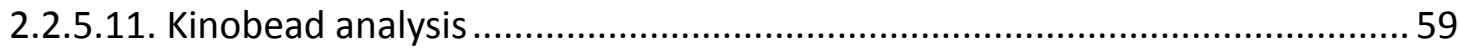

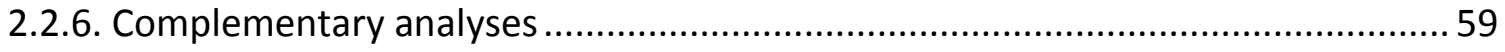

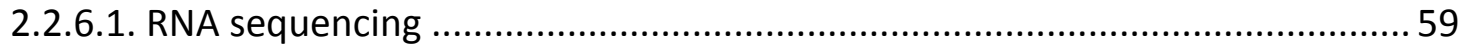

2.2.6.1.1. Sample preparation and data analysis .................................................. 59

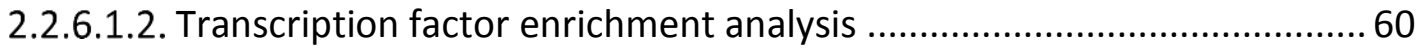

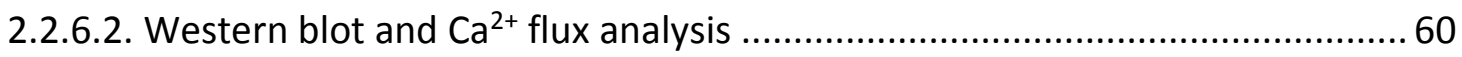

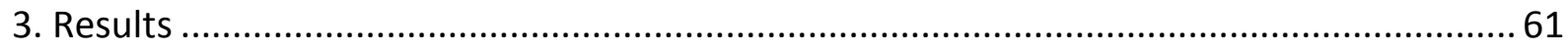

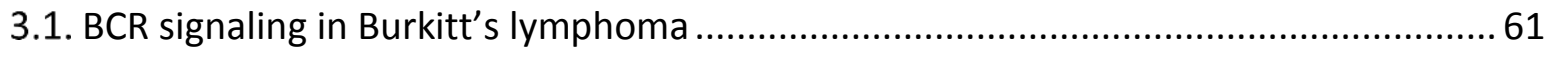

3.1.1. Strategy for the phosphoproteomic analysis of BCR signaling ............................62

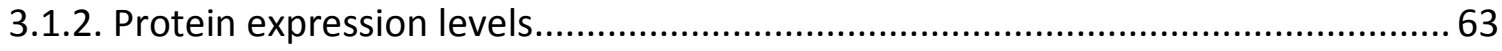

3.1.3. Identification, quantitation and regulation of phosphorylation sites ................... 64

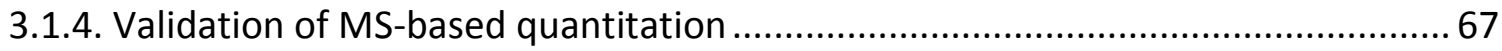

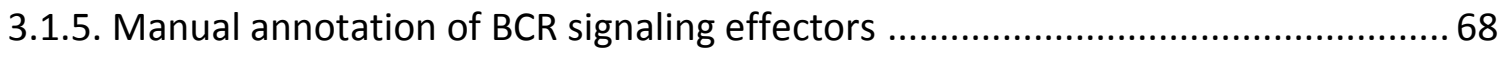

3.1.6. Network analysis of BCR-dependent phosphorylation sites................................69

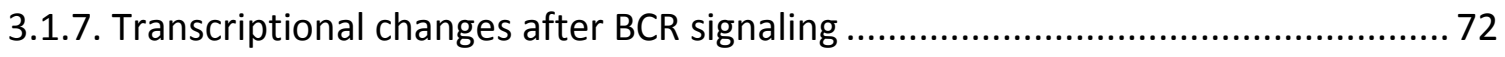

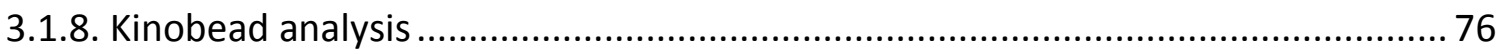

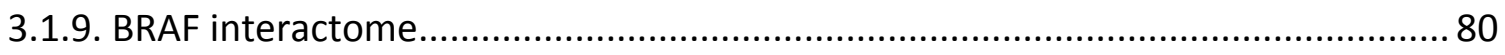

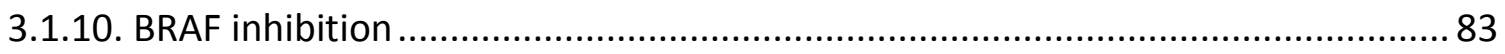

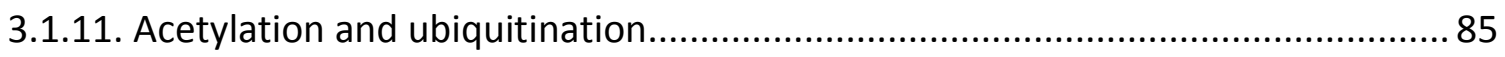

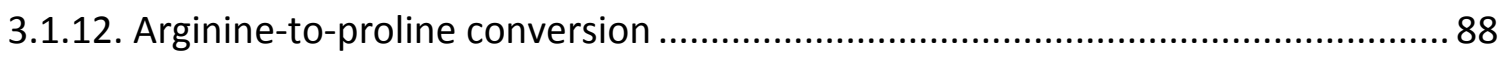

3.1.13. Antigen-dependent BCR signaling in three distinct Burkitt's lymphoma cell lines 91

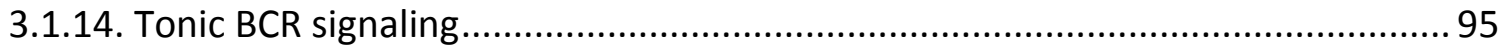

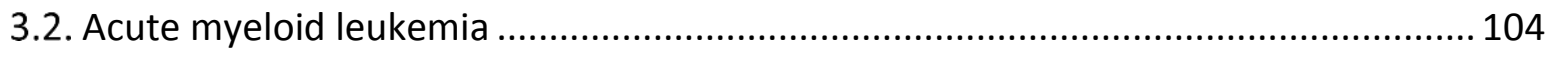




\section{Content}

3.2.1. BTK-dependent phosphoproteome networks in AML ................................... 105

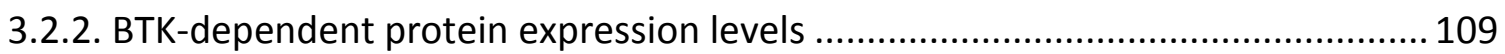

3.3. Phosphorylation dynamics in TCR signaling adapters .......................................... 111

3.3.1. SLP76/LCP2 phosphorylation site dynamics ..................................................... 111

3.3.2. GADS/GRAP2 phosphorylation site dynamics............................................... 113

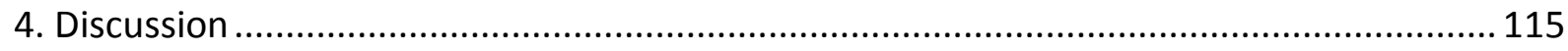

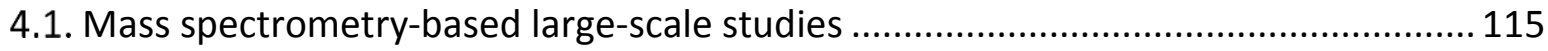

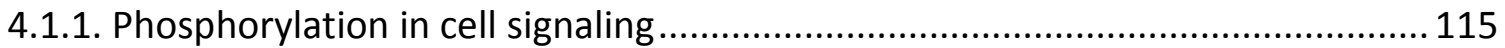

4.1.2. Acetylation and ubiquitination in cell signaling ............................................. 116

4.1.3. Large-scale B-cell receptor signaling studies ................................................... 117

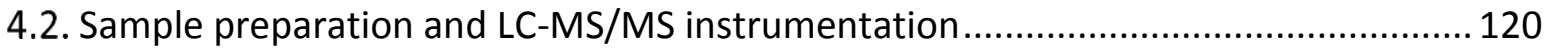

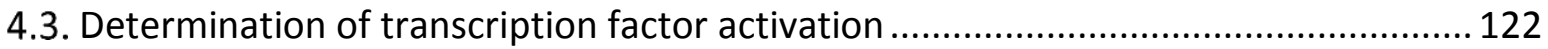

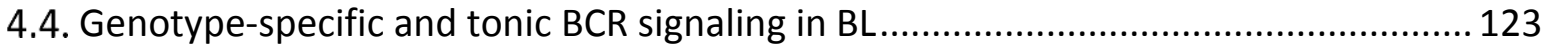

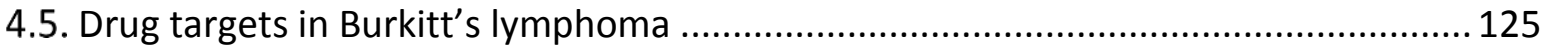

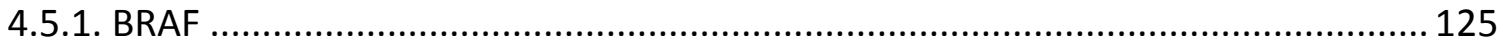

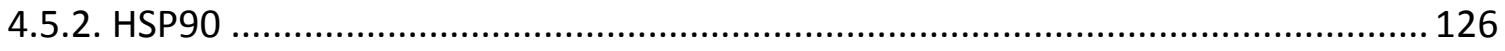

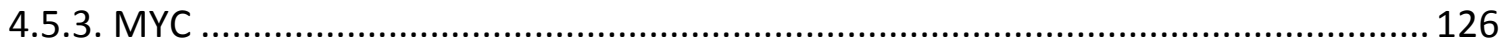

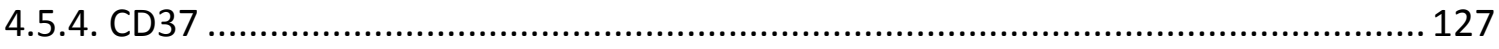

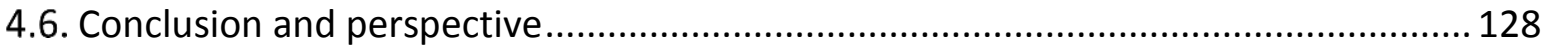

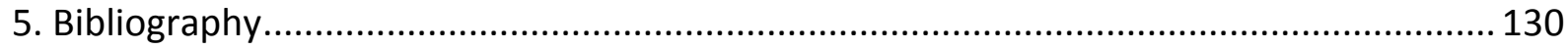

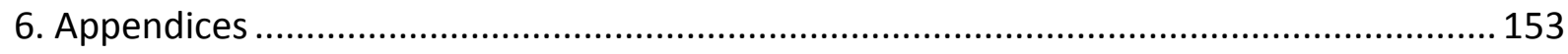




\section{Content}

\section{List of Figures}

Figure 1.1: Antigen-dependent and -independent B-cell receptor signaling ......................... 5

Figure 1.2: Mutations in functional protein categories and their cooperation in AML........... 10

Figure 1.3: Nomenclature of fragment ion species from peptide backbone breakage........... 15

Figure 1.4: Outline of generic building blocks of a bottom-up/shotgun proteomics workflow

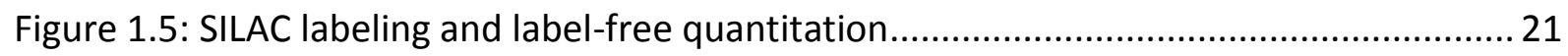

Figure 1.6: Post-translational modifications investigated in this work ................................. 24

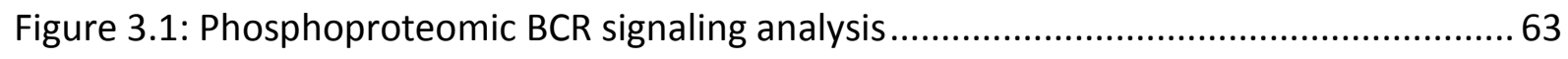

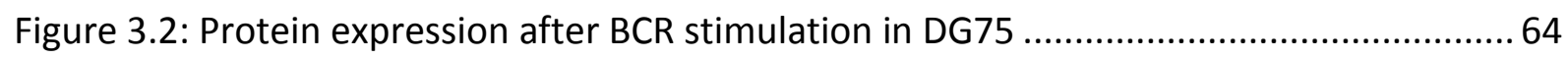

Figure 3.3: Phosphoproteome dynamics in DG75 after BCR stimulation .............................. 66

Figure 3.4: Validation of key phosphorylation sites in BCR signaling ................................... 68

Figure 3.5: Manual annotation of regulated BCR effector proteins in DG75 cells ................. 69

Figure 3.6: BCR effector protein-protein interaction networks............................................ 72

Figure 3.7: Time-dependent transcriptome analyses of BCR signaling ................................ 75

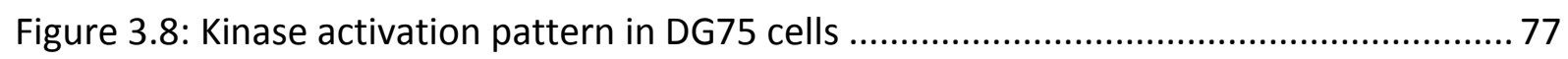

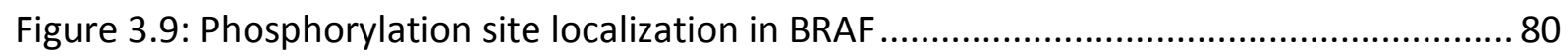

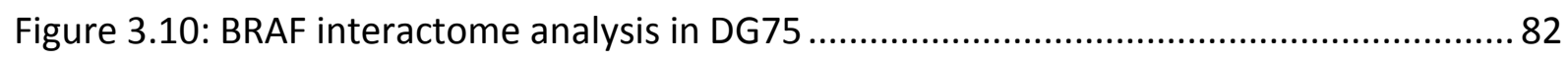

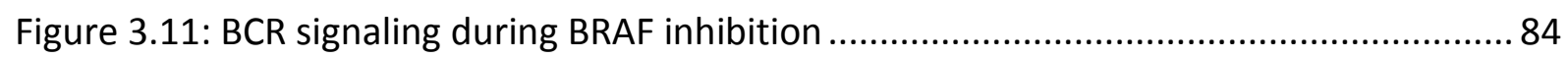

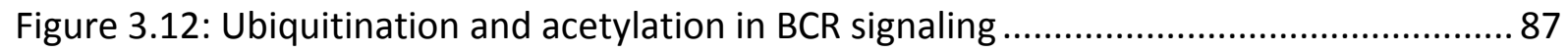

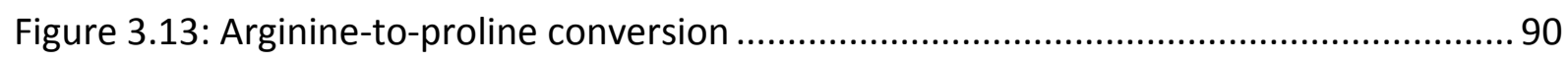

Figure 3.14: Antigen-dependent BCR signaling in three distinct BL cell lines........................93

Figure 3.15: Antigen-independent and early antigen-dependent BCR signaling ...................97

Figure 3.16: Differential regulation in antigen-dependent and tonic BCR signaling ............. 101

Figure 3.17: Phosphorylated tyrosine dynamics after BTK inhibition.................................. 106

Figure 3.18: Global phosphoproteomic analysis after BTK inhibition in AML ..................... 108

Figure 3.19: Common regulated sites in KG1 and MV4-11............................................ 109

Figure 3.20: Protein expression changes after BTK inhibition in AML................................ 110

Figure 3.21: Phosphorylation dynamics in the TCR signaling mediators SLP76 and GADS ... 112

Figure 4.1: Comparison of $p$-sites after 5 min of BCR stimulation in mouse and human B cells 


\section{Content}

\section{List of Tables}

Table 1: Overview of commonly applied label-based and label-free relative and absolute

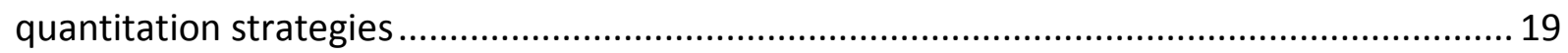

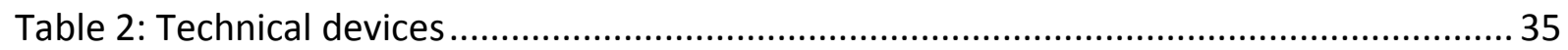

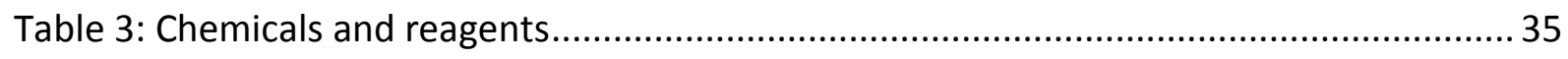

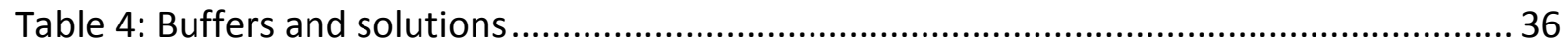

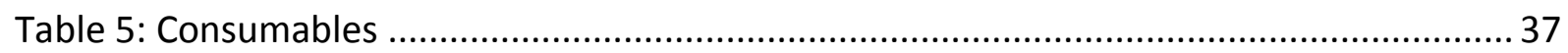

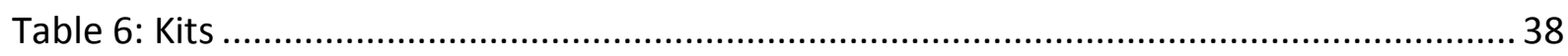

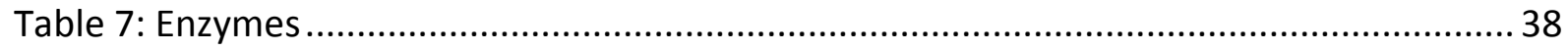

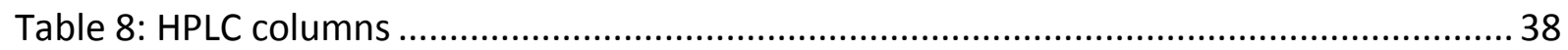

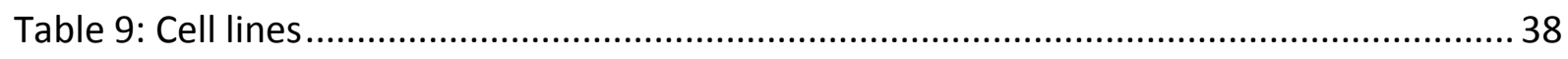

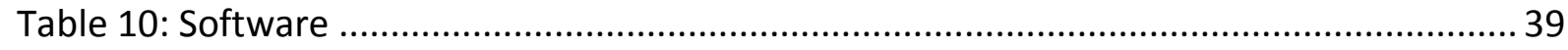

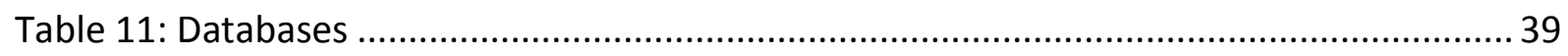

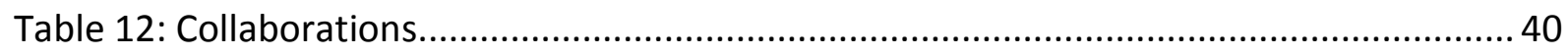

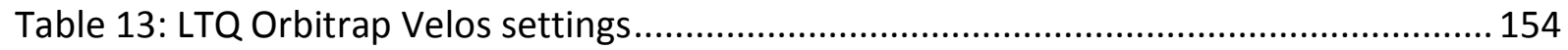

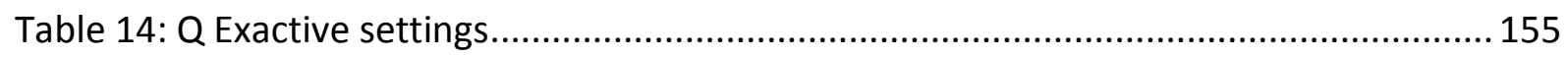

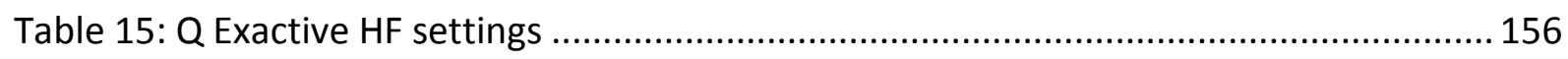

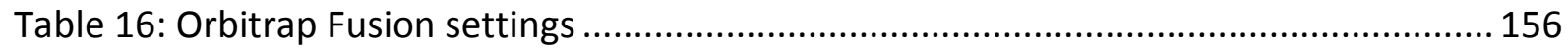




\section{Abstract}

Protein phosphorylation plays an important role in transmitting cellular signals. Phosphorylation has been mostly characterized by hypothesis-driven and low-throughput approaches in the past. However, novel phosphorylation sites on not yet identified signaling effectors involved in cellular responses can only be investigated in an unbiased and large-scale manner. The global investigation of phosphorylation processes in a cellular system can help to understand signaling that drives malignant transformation of cells. Moreover, phosphorylation patterns might hint towards potential therapeutic targets e.g. activated kinases. The generation and analysis of large-scale PTM datasets was made feasible by improved enrichment protocols in combination with the fast evolution of high resolution, quantitative mass spectrometry, computational approaches, and functional databases. The data presented in this thesis comprises three large-scale phosphoproteomics projects.

The main project represents the investigation of antigen-dependent and tonic BCR signaling in Burkitt's lymphoma (BL). As antigen-dependent BCR signaling in BL has not yet been described, phosphorylation changes were investigated in a time-resolved manner in DG75 cells and additionally in two other BL models including one primary BL cell culture. Overall, the MS-based antigen-dependent signaling analyses lead to the identification of thousands of altered phosphorylation sites upon BCR engagement in DG75. Based on this data, a higher order organization structure was extracted, complemented with database information and mapped to datasets derived from RNAseq, kinobead and kinase inhibition analyses. Signaling in BL cells was found to be highly individual in different cell lines. Tonic BCR signaling was investigated upon knockdown of the BCR signaling molecule CD79A and by inhibition of the receptor-proximal tyrosine kinase SYK. Comparison of the antigen-independent and dependent BCR signaling phosphoproteomes revealed substantial differences as well as certain similarities of both processes. The second project focused on the analysis of global phosphorylation dynamics in acute myeloid leukemia (AML) after BTK inhibition. As BTK was identified as an activated kinase in $A M L$, the mechanism of its activation and the influence of BTK ablation were investigated in two AML cell lines with different genetic backgrounds. Phosphoproteomic changes observed upon BTK inhibition highlighted individual differences and similarities of BTK action and identified relevant upstream activators of BTK as well as downstream effector proteins in AML cells. The third project was aimed at profiling the phosphorylation site dynamics of the TCR adapter proteins LCP2 (SLP76) and GADS (GRAP2) 


\section{Abstract}

in time-resolved TCR stimulation. The dynamics of several phosphorylation sites could be captured over a time course, and provide a basis for further functional characterization by e.g. mutation of p-sites. In summary, this work globally characterized phosphorylation changes upon different cellular conditions by quantitative mass spectrometry-based phosphoproteomics and provides a basis for the identification of druggable proteins or yet unknown signaling effectors. 


\section{Introduction}

\section{1. $B$ cell and $T$ cell signaling}

The human immune system is a highly complex defense system composed of different cells, tissues and organs that protect the human body from pathogens. Two of its major cell types, $B$ and T cells are important cellular effectors of the adaptive immune system (Murphy, 2011). B-cell development and maturation are tightly regulated by various differentiation processes to produce a naïve B cell with a functional, monospecific B-cell antigen receptor (BCR) (Nossal and Lederberg, 1958; White, 1958). Terminally differentiated B cells execute unique functions in the immune system as either plasma cells (PC) or long-lived memory B cells. PCs secrete antibodies whereas memory B cells enable fast, high-affinity secondary immune responses i.e. after re-exposition to their cognate antigen or during vaccination (Gray and Skarvall, 1988) (reviewed in Klein and Dalla-Favera, 2007). As all hematopoietic cells, B cells arise from common pluripotent hematopoietic stem cells that reside in the bone marrow and can differentiate into either common myeloid or common lymphoid progenitor cells. Common lymphoid progenitor cells give rise to all cells of the lymphatic lineage: B cells, T cells and natural killer (NK) cells. Human B cells develop and mature in the bone marrow. Naïve B cells egress from the bone marrow niche to circulate through secondary lymphoid organs (such as lymph nodes, spleen, tonsils and Peyer's patches in the small intestine) until activation via their B-cell receptor (BCR) by a cognate antigen (Harwood and Batista, 2011). Both B and T cells belong to the lymphoid lineage of hematopoietic cells. In contrast to B cells, T cells mature in the thymus, before circulating as naïve T cells in the secondary lymphoid organs.

\subsubsection{B-cell receptor signaling}

The BCR exerts two functions in B cells: first, it ensures B-cell development, survival and homeostasis independently of antigens, also referred to as tonic BCR signaling. Second, the $B C R$ recognizes foreign antigens and subsequently induce characteristic $B$ cell processes (reviewed in Kurosaki et al., 2009; Niiro and Clark, 2002; Rickert, 2013). The BCR is composed of two membrane-anchored parts: a ligand-binding, transmembrane immunoglobulin part (Ig) and the non-covalently associated, intracellular signaling chains CD79A and CD79B (Ig $\alpha$ and $\operatorname{Ig} \beta)$ (Reth, 1989). The Ig molecule consists of two Ig heavy (IgH) and two Ig light (IgL) chains 
which are linked to each other by disulfide bonds (Figure 1.1). Both, the IgH and the IgL part can be separated into a variable, antigen-binding domain and a constant region. The constant region of the IgH chain determines the antibody class such as IgM or IgG (Borst et al., 1996). The antigen-binding domain consists of a variable (V), diversity (D; only in the heavy chain) and joining $(\mathrm{J})$ gene segment. For each segment, various variants are encoded at the respective gene locus that can be combined in a random fashion. Therefore the BCR can bind to a large variety of antigens (Brack et al., 1978).

Almost all signals derived from the BCR are transmitted via the intracellular signaling chains CD79A and CD79B harboring a so-called immunoreceptor tyrosine-based activation motif (ITAM; Figure 1.1) (Engels et al., 2009; Reth and Wienands, 1997). The ITAM motif consists of two tyrosine residues in a conserved amino acids stretch (Reth, 1989). Antigen-independent tonic BCR signals dependent critically on the ITAM motif as its ablation leads to cell death of mature B cells (Kraus et al., 2004; Lam et al., 1997a). Compared to antigen-dependent signaling, tonic signaling is less well understood, and believed to transmits baseline signals mainly via PI3K signaling (Figure 1.1, right panel) (Srinivasan et al., 2009; Young and Staudt, 2013). The initial step in antigen-dependent signaling is mediated by receptor engagement by antigen binding that leads to the formation of BCR-CD79A/CD79B clusters in the membrane (reviewed in Monroe, 2006). Phosphorylation of the two tyrosine residues in the ITAM motif is mediated by Src family kinases (SFKs) such as LYN after receptor engagement. The phosphorylated tyrosine residues provide a binding site for the tandem Src homology 2 (SH2) domains of the Spleen tyrosine kinase (SYK) leading to its activation (Figure 1.1, left panel). 


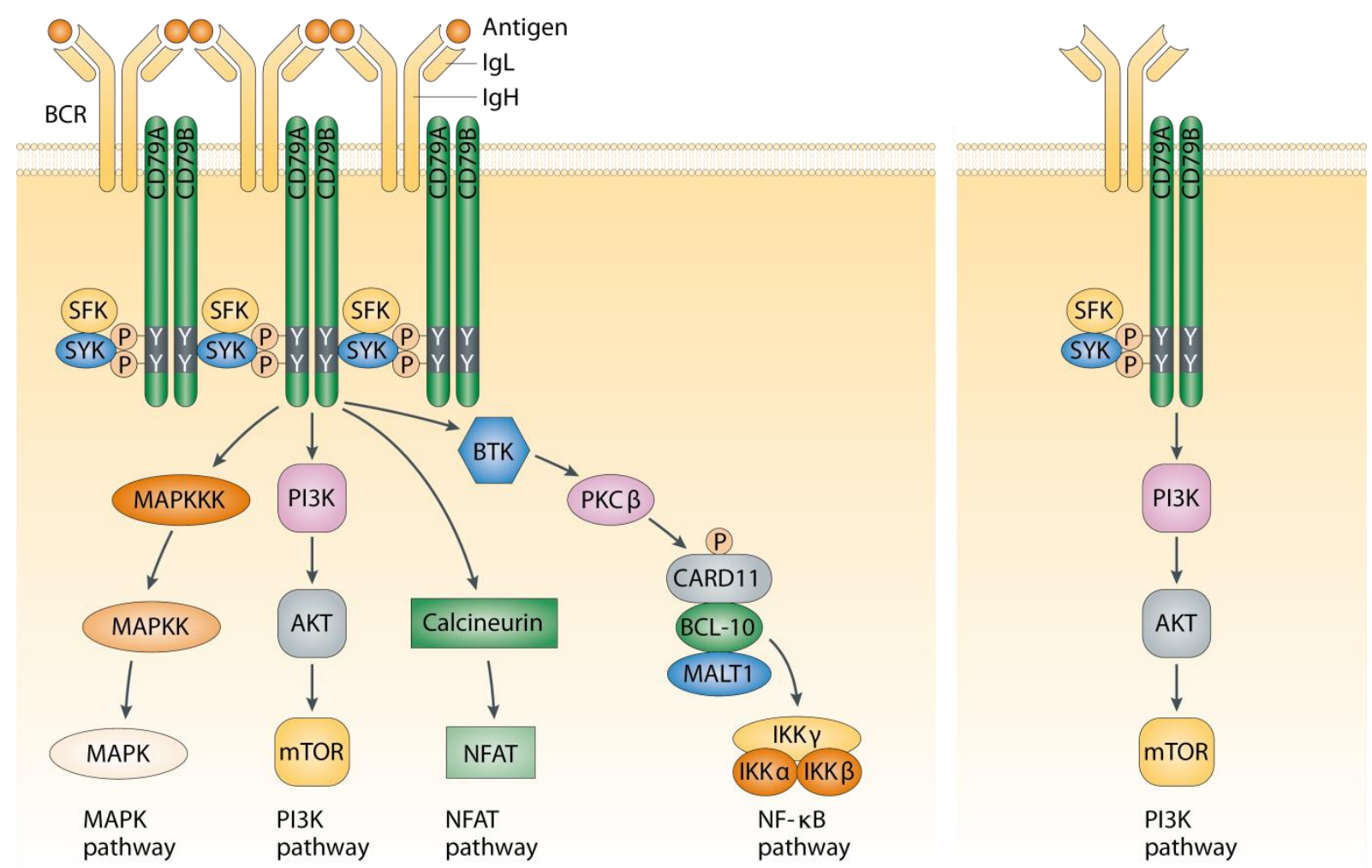

Figure 1.1: Antigen-dependent and -independent B-cell receptor signaling

The left panel shows the signaling cascade after cognate antigen encounter of the BCR complex. Src family kinases (SFK) such as LYN phosphorylate the ITAM motifs of the CD79A/B signaling chains thereby providing a binding platform for SYK that leads to its activation. After activation, several downstream effectors (CIN85, BLNK, BTK, PLCY, PI3K, PKC $\beta$ and the CARD11BCL-10-MALT1-complex) that drive distinct signaling pathways (MAPK, PI3K, NFAT and NFKB) eventually become activated. The right panel shows tonic BCR signaling involving mainly PI3K signaling. The figure is adapted from Young and Staudt, 2013.

SYK phosphorylates in the following various effector proteins (adapter proteins, phospholipases, kinases and other enzymes) such as BLNK, BTK, and PLCy2 (Figure 1.1, left panel). BCR signaling also involves the generation of second messengers like $\mathrm{Ca}^{2+}, \mathrm{IP}_{3}$ and $\mathrm{DAG}$. Together these processes lead to the formation of BCR-dependent signalosomes that trigger the initiation of various signaling cascades i.e. NFKB-, PI3K-, NFAT- and MAP-kinase-signaling (Figure 1.1, left panel; reviewed in Kurosaki et al., 2009; Young and Staudt, 2013). In response to the membrane-associated antigen encounter, the BCR-antigen complex is internalized, the antigen becomes processed in the late endosome and coupled to major histocompatibility complex (MHC) class II molecules. The MHC-II complex is subsequently exported to the B cell surface for co-stimulatory interaction with cognate $\mathrm{T}$ cells, which is a pre-requisite for the formation of a germinal center (GC) (reviewed in Kurosaki et al., 2009; Rickert, 2013). Germinal centers are histological structures that are formed in secondary lymphoid organs and can be functionally divided into two parts: a light zone with mainly centrocytes, follicular dendritic cells and T follicular helper cells, and a dark zone with centroblasts. The dark zone is characterized by high proliferation and somatic hypermutation whereas the light zone 
centrocytes are selected for high affinity lgs. During somatic hypermutation, base-pairs are exchanged in the $V(D) J$ region of the IgV genes (reviewed in Küppers, 2005). Centrocytes with a high antigen-binding affinity undergo Ig class-switch recombination of the immunoglobulin heavy chain. During class-switch recombination the isotype of the BCR is switched from $\lg M / \lg D$ to $\lg G$, $\lg A$ or $\lg E$ thereby mediating different effector functions. Somatic hypermutation as well as class-switch recombination require the activity of the activationinduced deaminase (AID) (reviewed in Rickert, 2013). GC B cells can switch between light and dark zone thereby changing their gene expression pattern. Eventually, centrocytes differentiate into long-lived plasma cells (PC) or memory B cells (reviewed in Küppers, 2005).

\subsection{2. $T$ cells and TCR signaling}

Same as B cells, T cells possess a membrane-bound antigen receptor, the T-cell receptor (TCR) which is composed of two hetereodimeric variable TCR receptor chains (TCR $\alpha$ and $\beta$ ) associated with three different dimeric CD3 signaling modules, CD3 $\delta / \varepsilon, C D 3 \gamma / \varepsilon$ and CD3 $\zeta / \zeta$. The accessory CD3 chains contains as CD79A and B ITAM motifs in their intracellular domains. In contrast to the BCR, the TCR does not exist in a soluble form, has only one antigen-binding site, and does solely bind to antigen-MHC complexes and not to soluble antigens (Garcia and Adams, 2005; Hwang et al., 2015). Dependent on the MHC class (I or II) the antigen is presented on, T cells with either CD4 or CD8 co-receptors become activated and differentiate in specialized T cell effector classes. $\mathrm{CD} 8^{+}$cells differentiate into cytotoxic $\mathrm{T}$ cells (CTL) that directly eliminate virus-infected cells whereas $C D 4^{+} \mathrm{T}$ cells differentiate in various $\mathrm{T}$ helper classes like $T_{H} 1, T_{H} 2, T_{H} 17$ or $T$ follicular helper $\left(T_{F H}\right)$ cells. Especially $C D 4^{+} T_{H} 1, T_{H} 2$ and $T_{F H}$ provide a co-stimulatory signal to antigen-activated $B$ cell for further differentiation (reviewed in Samelson, 2011).

The signaling cascades initiated upon $\mathrm{MHC} /$ antigen recognition on the cell surface leading to T cell responses are in principle similar to the ones in B cells, as in both cases they are mainly mediated by phosphorylation. The processes and the involved adapter proteins and kinases are also very similar. The earliest events after cognate antigen encounter is the activation of the Src family kinase LCK, phosphorylating the ITAM motifs in the CD3 chains (Chakraborty and Weiss, 2014; Guy et al., 2013). ZAP70, a SYK family kinase primarily expressed in T cells, binds with its tandem $\mathrm{SH} 2$ domain to the doubly tyrosine phosphorylated ITAM-motif and thereby becomes activated (reviewed in Samelson, 2011). Downstream phosphorylation of 
ZAP70 targets the adapter proteins SLP76 (LCP2) and membrane-attached LAT which provides a binding platform and therefore leads to the recruitment of other adapter proteins (Balagopalan et al., 2010; Myung et al., 2001). The SLP76/LAT-nucleated complex activates other adapter proteins (amongst others GADS and GRB2) and leads to the generation of second messenger molecules (reviewed in Huang and Sauer, 2010). Downstream events of TCR signaling are transcriptional activation and cytoskeletal changes leading to the aforementioned, defined T cell responses.

\subsection{Hematologic malignancies - leukemia and lymphoma}

Hematological malignancies comprise a huge class of neoplasms derived from cells of the hematopoietic lineage that affect the blood, bone marrow, and the lymphatic system. There are two major classes of hematological malignancies: liquid tumors - leukemias and solid tumors - lymphomas. Leukemias are characterized by massive proliferation of immature cells (blasts) in the bone marrow that outcompete normal hematopoiesis and eventually spread out to the blood. Dependent on the malignant transformed cell type, they can be classified as of myeloid or lymphatic origin. Leukemias can be classified into chronic or acute clinical forms: Chronic leukemias spread more slowly and can stay undiagnosed for several years while acute leukemias manifest within a short time (weeks or months) (reviewed in Estey and Döhner, 2006; Koeffler and Golde, 1978). Lymphomas comprise a heterogeneous entity of tumors derived from lymphatic cells that arise in lymphatic tissues (Campo et al., 2011). They can be divided into Hodgkin lymphomas with their characteristic Hodgkin-Reed-Sternberg-Stern (HRS) cells, and the heterogeneous group of non-Hodgkin-lymphomas (NHL). Hodgkin lymphomas are exclusively derived from B cells whereas NHL neoplasia can be either derived from B or T cells, although the B-cell derived disease is more common (reviewed in Shaffer et al., 2012; Young and Staudt, 2013a). NHLs can be divided into slow-growing indolent, or rapidproliferating, aggressive lymphomas. Different types of NHL exhibit features (gene expression profile, surface markers, the occurrence of somatic hypermutation or class-switch recombination) that can be attributed to a defined developmental stage of healthy precursor B cells (reviewed in Shaffer and Staudt, 2012). Two hematological malignancies were intensively analyzed in this work and will be described in further detail: Burkitt's lymphoma and acute myeloid leukemia. 


\subsubsection{Burkitt's lymphoma}

Burkitt's lymphoma (BL) is an aggressive NHL derived from B cells. The disease was first described in 1958 in Uganda, Africa by the physician Dennis Burkitt in children with jaw 'sarcomas' (Burkitt, 1958). Two years later, BL was identified to be of lymphatic origin and in 1964 its association with Epstein-Barr virus (EBV) was detected (Epstein and Barr, 1964; O'Conor and Davies, 1960). The connection between the disease and the causative viral agent turned out to be one of three clinical variants of BL: EBV-associated endemic variant (eBL), the immunodeficiency-associated (hivBL) and the sporadic form ( $\mathrm{sBL}$ ). The endemic form is also associated with malaria infection and is geographically correlated to EBV- and malariaendemic regions (Burkitt, 1961; 1962). The immunodeficiency-associated form occurs exclusively in combination with human immunodeficiency-associated virus (HIV) infection (Kalter et al., 1985). The sporadic form is evenly distributed all over the world and the most dominant BL type in non-malaria regions. BL tumors grow fast and their localization varies between the clinical variants: in the sporadic form they are primarily intraabdominal, in the endemic form mainly in the jaw, face or neck (reviewed in Molyneux et al., 2012). The three forms are hardly distinguishable by morphology and immunophenotyping. The histological appearance of BL specimen is the characteristic 'starry sky' present in the bone marrow and lymph nodes. The 'starry sky' is caused by macrophages containing dead apoptotic tumor cells (reviewed in Ferry, 2006). Tumor B cells are of intermediate size with round nuclei, multiple nucleoli and a high proliferation index (reviewed in Fujita et al., 2004a; 2004b; Nakamura et al., 2002). The standard treatment for BL is classical chemotherapy. Although $B L$ is very chemosensitive and modern multiagent treatment regimens show a good cure rate, the treatment has severe toxic side effects that may not be well tolerated by older patients or require intensive supportive care (Hoelzer et al., 2014; Rizzieri et al., 2004). Rituximab, a monoclonal antibody directed against the B cell-specific surface marker CD20 was added as a therapeutic option to classical chemotherapeutic agents that act by introducing DNA strand breaks, DNA intercalation, microtubule inhibition and immunosuppression (Rizzieri et al., 2014).

The karyotype of Burkitt's lymphoma cells is of low complexity and the BL gene expression pattern resembles that of healthy germinal center B cells (Dave et al., 2006; Seitz et al., 2011). Of special importance in this gene expression program is the activation-induced deaminase (AID). The AID mediates somatic hypermutation in the variable antigen-binding domain 
occurring in the dark zone and class-switch recombination of the immunoglobulin constant region in the light zone of the GC (reviewed in Rickert, 2013). The hallmark of BL is an Ig-MYC translocation caused by the action of the AID (Bergsagel et al., 1996; Neri et al., 1988; Schmitz et al., 2014). MYC is a transcription factor and oncogene involved in many cellular processes such as metabolism, cell growth, proliferation and apoptosis (reviewed in Cai et al., 2015; Dang, 2012). MYC presumably increases the expression of all active genes at a given time rather than imposing a particular gene expression program on a cell (Lin et al., 2012; Nie et al., 2012). In non-oncogenic cells, MYC expression is tightly controlled and aberrant expression leads to apoptosis (Evan et al., 1992; Felsher and Bishop, 1999; Gibson et al., 1995; Kanazawa et al., 2003; Lowe et al., 2004; Nilsson and Cleveland, 2003). The BL associated translocation brings $I g$ genes with their enhancer regions in close proximity to the transcription factor MYC leading to its constitutive overexpression (Dalla-Favera et al., 1982; Taub et al., 1982). BL cells circumvent MYC-induced apoptosis by additional mutations in genes that cooperate with MYC-mediated proliferation (Cai et al., 2015; Srinivasan et al., 2009). The most common IgMYC translocation variant is $(8 ; 14)(q 24 ; q 32)$ where $M Y C$ is brought under the transcriptional control of the $\mathrm{IgH}$ locus on chromosome 14. The other two less frequent variants include MYC fusions with Ig light chains (reviewed in Ferry, 2006). In BL patients there is an overall MYC mutation rate of 70\% (Bahram et al., 2000; Schmitz et al., 2012). Other common mutational events affect the transcription factor TCF3 and its negative regulator ID3. TCF3 encodes the transcription factor E2A which is expressed in GC centroblasts and regulates the expression of immunoglobulin genes and other B cell-specific genes (Schmitz et al., 2012; 2014). Both mutations in the TCF3/ID3-module circumvent the inactivation of TCF3. Moreover, the cell cycle regulator cyclin D3 (encoded by CCND3) and the tumor suppressor TP53 are affected (Küppers, 2005; Schmitz et al., 2012; 2014). Although BL cells depend on a functional BCR, the disease is thought to be driven by tonic, antigen-independent BCR activation indicated by activation of the PI3K signaling pathway rather than the antigen-dependent NFKB involvement (Figure 1.1, right panel) (Dave et al., 2006; Schmitz et al., 2012; Young and Staudt, 2013b).

\subsubsection{Acute myeloid leukemia}

Acute myeloid leukemia ( $A M L)$ is derived from transformed hematopoietic progenitor cells (blasts) and is characterized by a huge variety of genetic mutations. AML blasts are derived from a clonal hierarchy and proliferate fast and uncontrollably in the bone marrow. The symptoms of AML are caused by the replacement of normal hematopoiesis in the bone 
marrow. This leads to a decrease in erythrocyte, thrombocyte and leukocyte cell numbers and results in anemia, an increased risk of bleeding and vulnerability to infections due to a compromised immune system (reviewed in Chen et al., 2013; Czibere et al., 2006; Estey and Döhner, 2006). A common risk factor for AML is age, but there are also predisposing factors such as trisomy 21 , exposition to ionizing radiation, chemicals such as benzene or previous chemotherapeutic treatment with cytostatic drugs (reviewed in Chen et al., 2013; Czibere et al., 2006; Estey and Döhner, 2006). Treatment options for AML consist of different chemotherapy regimens possibly followed by allogeneic hematopoietic stem cell transplantation. In general, chemotherapeutic treatment is highly toxic and therefore not well tolerated. The success of the treatment strategy also correlates with patient age and the mutational profile of the disease (reviewed in Czibere et al., 2006). In recent years, a huge effort was made to gain insights into AML biology, to characterize the impact of different disease genotypes on prognosis and to identify efficient targeted treatment strategies. Different recurrent mutations and gene fusions were identified in nine functional protein classes involving genetic aberrations in transcription factors, NPM1, tumor suppressor genes, epigenetic modifier genes, signaling genes, myeloid transcription factor genes, cohesin- and spliceosome-complex genes (Figure 1.2) (Chen et al., 2013).

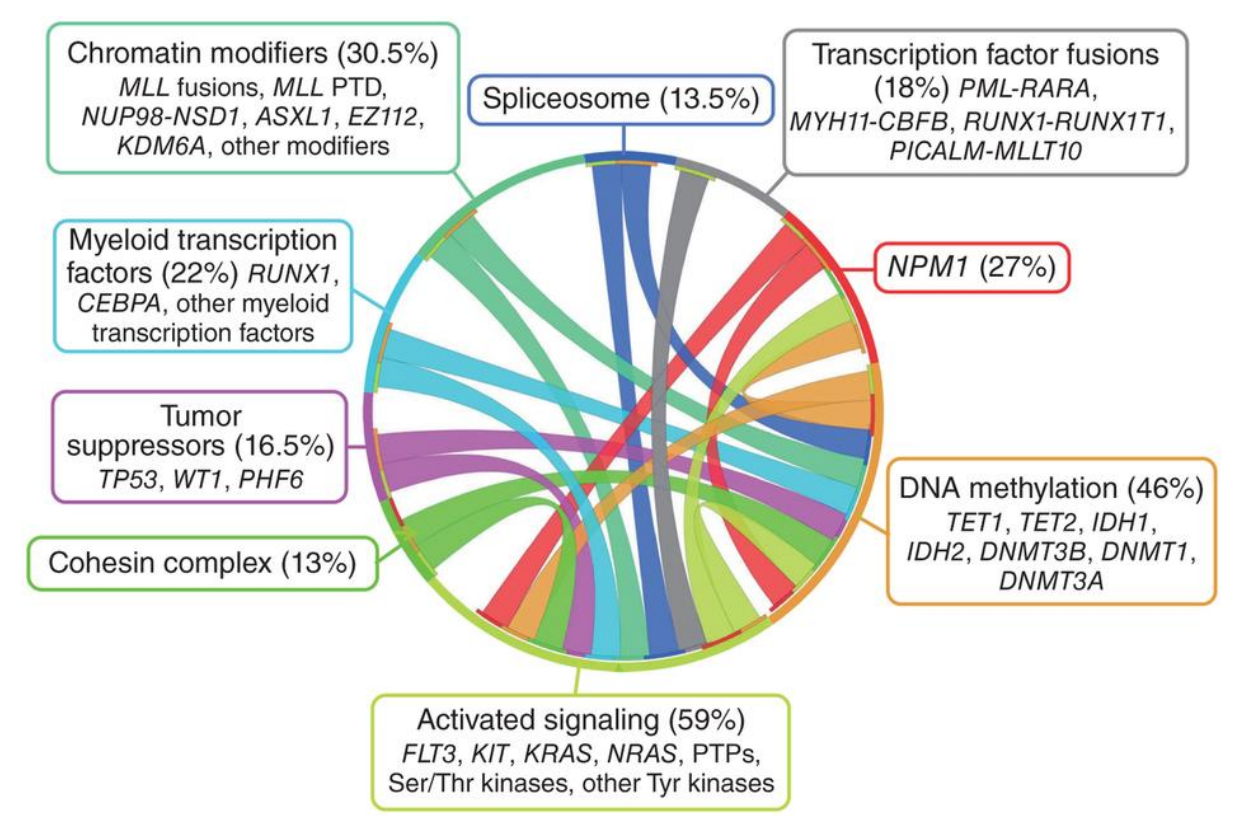

Figure 1.2: Mutations in functional protein categories and their cooperation in AML

The Circos plot shows co-occurring mutations in AML. Proteins are grouped into nine different functional groups (represented by different colored boxes). Co-occurring mutations between these groups are illustrated by ribbons connecting those groups. The figure is published by Chen et al., 2013. 


\subsection{Mass spectrometry-based proteome and PTM analysis}

The underlying cause of lymphoma and leukemia, or in general cancer can be often traced back to genetic alterations that manifest themselves at the protein or post-translational modification (PTM) level, such as elevated protein expression or altered phosphorylation. The proteome is defined as the complete set of proteins expressed from a genome in a specimen i.e. on the cellular or subcellular level at any given time point (Wilkins et al., 1996). In contrast to the genome, the proteome is more diverse as genes can exist on the protein level in various isoforms and additionally can be post-translationally modified (Wilkins et al., 1996). Mass spectrometry became a powerful tool for the high-throughput characterization of proteins and PTMs within the last years. It offers the possibility to investigate proteins and PTMs in a hypothesis-free, global and unbiased manner which makes it a valuable tool for the analysis of cancer proteomes and signaling (reviewed in Mann and Jensen, 2003).

\subsubsection{Tandem mass spectrometry}

For unambiguous protein identification it is necessary to identify a unique peptide derived from that particular protein. Solely the mass of the intact peptide is not enough information for its unambiguous identification, especially in highly complex samples. For unambiguous peptide identification it is therefore necessary to deduce its primary amino acid sequence. The addition of a PTM is accompanied by a mass increase of the peptide and, in most cases its fragment ions. From these sequence specific fragment ions, the position of the PTM on the primary amino acid sequence can be determined (reviewed in Mann and Jensen, 2003b). Tandem mass spectrometry is a suitable tool for the identification of proteins and the localization of PTMs. In general, a mass spectrometer is an analytical instrument that measures the mass-to-charge $(\mathrm{m} / \mathrm{z})$ ratio and the intensity of ions in the gas-phase. Tandem mass spectrometry or (commonly referred to as MS/MS) combines the measurement of an intact peptide mass followed by its fragmentation for the determination of the peptide amino acid sequence (Steen and Mann, 2004). After fragmentation of the precursor, the localization of the PTM along the amino acid chain can be determined at the MS/MS level by the mass increase of the fragment ion (reviewed in Mann and Jensen, 2003b).

Tandem mass spectrometry can be performed either in instruments containing distinct mass analyzers for separation, fragmentation and fragment analysis, commonly referred to as tandem-in-space such as performed by triple quadrupoles, quadrupole-time-of-flight or 
orbitrap instruments. Instruments where all the before mentioned steps are performed in one device are referred to as tandem-in-time instruments for instance Fourier transform ion cyclotron resonance (FT-ICR) or ion trap instruments (Gross, 2011). In tandem mass spectrometry, as a first step, the peptide mass-over-charge $(\mathrm{m} / \mathrm{z})$ ratio or Thomson of all peptides eluting from chromatographic separation at a given time point is determined in a survey or MS1 scan. Precursor peptides are selected in a first mass filter and fragmented into smaller parts, ideally along the peptide backbone. A product ion scan (alternatively MS/MS or MS2) experiment is then performed on the peptide precursor, giving information about the amino acid composition. In modern tandem mass spectrometers, the precursor selection for the MS2 scan is data driven and occurs in an automated fashion, the so-called data-dependent acquisition (DDA). In DDA, the top $\mathrm{N}$ most abundant precursor peptides from a survey scan are consecutively extracted from all other precursors for fragmentation for the whole duration of the chromatographic separation. Moreover, precursor ions are selected for fragmentation at their elution apex to decrease ion accumulation time and to improve MS2 spectra quality. The software can also automatically recognize if a precursor ion is likely to be derived from a peptide according to its isotopic pattern (averagine model of an amino acid; Senko et al., 1995). Although these features were designed to increase peptide identification rates, the precursor selection is of stochastic nature. This leads to some variability between replicate samples and a bias towards high abundant peptides at the expenses of low abundant species which are less likely to become analyzed (Liu et al., 2004; Michalski et al., 2012). A recent trend to overcome these drawbacks is the development and establishment of data-independent acquisition (DIA). In DIA methods, peptides over the whole $\mathrm{m} / \mathrm{z}$ range are subjected consecutively with a defined $\mathrm{m} / \mathrm{z}$ window range to fragmentation independently of the precursor intensity (Egertson et al., 2013). The method of choice for data generation in this work was DDA and thus will be explained in more detail in section 1.3.5.

\subsubsection{Sample ionization}

The foundation for the success of mass spectrometry was laid by the inventions of matrixassisted laser desorption ionization (MALDI) and electrospray ionization (ESI). MALDI and ESI are both soft ionization techniques, leaving biomolecules like peptides and proteins intact during the ionization process. The gain for the scientific field was so fundamental and significant that the inventors John B. Fenn (ESI) and Koichi Tanaka (MALDI) were jointly awarded the Nobel Prize in 2002 (Fenn et al., 1989; 1990; Tanaka et al., 1988). In MALDI, the 
analyte molecules are desorbed from a surface into the gas phase by laser irradiation. The basic principle of ESI is a high electrostatic voltage between the tip of the capillary needle and the entrance of the mass spectrometer. The analyte-containing solution becomes dispersed from a cone that is formed on the capillary tip to form multiple charged droplets. Those droplets are attracted towards the orifice into the vacuum of the mass spectrometer. The solvent removal mechanism and the analyte ionization can be explained by two models: the charge-residue model and the ion evaporation model, respectively (reviewed in Mallick and Kuster, 2010). In the charge-residue model, the surface charge concentration of the droplet increases by continuous solvent evaporation. When the internal Coulombic repulsion becomes greater than the surface tension (Rayleigh limit), the droplet dissociates into smaller droplets with a lower charge. Eventually, this leads to an iterative series of solvent evaporation and explosions until single ions are formed (Dole et al., 1968). The ion evaporation model postulates that single ionized peptides emit from the surface of the charged droplet by field desorption (Thomson and Iribarne, 1979). The exact mechanism is still under debate and might be dependent on the analyte properties (Konermann et al., 2012). However, both models result in multiple positively charged ions in the gas phase which makes the peptides susceptible for manipulation as required for MS measurement (reviewed in Mallick and Kuster, 2010).

Over the years, ESI became the dominant ionization method due to the advantage of ESI over MALDI that samples get ionized out of a liquid phase which makes it directly compatible to reversed-phase high-performance liquid chromatography (RP-HPLC; in combination with MS/MS abbreviated as LC-MS/MS) (Fenn et al., 1990). A further advancement of ESI is nanoESI which utilizes lower flow rates of a few hundred $\mathrm{nL} / \mathrm{min}$. This leads to lower sample and mobile phase consumption, higher ionization efficiency due a slightly different ionization mechanism and therefore increased sensitivity (Karas et al., 2000; Schmidt et al., 2003).

\subsubsection{Peptide sequencing}

In tandem mass spectrometry the mass measurement of the peptide precursor is followed by its dissociation for the determination of the primary amino acid sequence (reviewed in Mann and Jensen, 2003b). The most commonly applied fragmentation techniques is collision induced dissociation (CID). In order to deduce the peptide amino acid sequence the analyte peptide ions are collided with inert gas such as helium (He), nitrogen $\left(\mathrm{N}_{2}\right)$ or argon ( $\left.\mathrm{Ar}\right)$. CID fragmentation technique typically generates $b$ - and $y$-ion species, where $b$-ions contain the 
peptide $\mathrm{N}$-terminus and $\mathrm{y}$-ions contain the peptide C-terminus, respectively (Figure 1.3) (Roepstorff and Fohlman, 1984). Two types of CID fragmentation can be distinguished: ion trap CID and beam-type CID which is also commonly referred to as higher energy collisional dissociation (HCD). The former, as the name suggests, is often performed in ion trap mass analyzers where the ions are activated in a radio frequency field. The peptide ions collide multiple times with He molecules thereby breaking along the peptide backbone at the peptide bonds generating typically $b$ - and y-ions (Figure 1.3). CID ion trap fragmentation follows the lowest energy pathway fragmentation (Jedrychowski et al., 2011; Michalski et al., 2012). In case the peptide carries a labile PTM like phosphorylation or glycosylation, this bond is likely to break leading to loss of small, neutral molecules such as $\mathrm{H}_{2} \mathrm{O}$ or $\mathrm{H}_{3} \mathrm{PO}_{4}$ which is commonly referred to as neutral loss (reviewed in Mallick and Kuster, 2010; Mann et al., 2002). Overall, this leads to insufficient fragmentation and therefore incomplete peptide sequence information (reviewed in Mallick and Kuster, 2010). Moreover, ion traps suffer from a low mass cut-off which means that ions with an $\mathrm{m} / \mathrm{z}$ of less than $30 \%$ of the precursor cannot be detected (Zhang et al., 2005).

An alternative to ion trap CID is beam-type CID that does not take place in the analyzer but in a separate collision cells e.g. collision multipole in Q Exactive instruments or in the ion-routing multipole in the Orbitrap Fusion. Compared to ion trap CID, peptide ions collide fewer times with collision gas molecules but also preferably generate peptide backbone cleavages leading to $b$ - and y-ions (Figure 1.3). The collisions happen on a higher kinetic energy regime compared to ion trap CID which reduces neutral losses of PTMs. After beam-type fragmentation, ions are accumulated and trapped before they are sent for analysis to a high resolution analyzer e.g. orbitrap or TOF (Michalski et al., 2012; Olsen et al., 2007). 


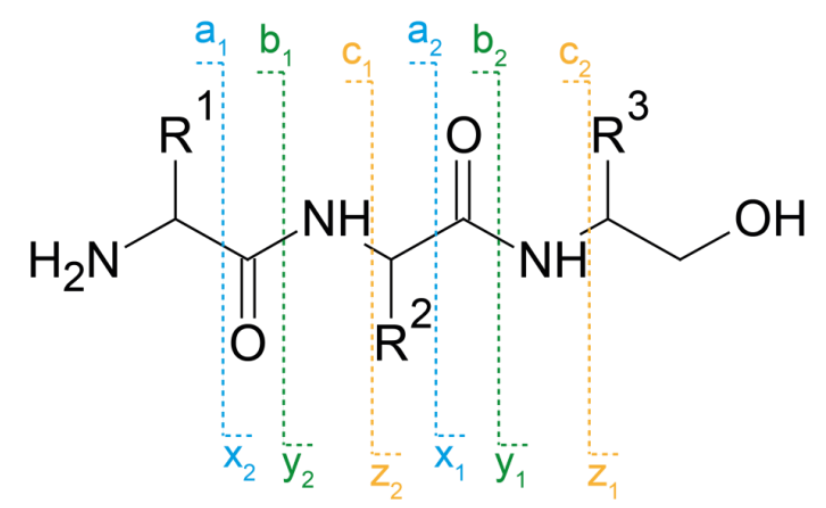

Figure 1.3: Nomenclature of fragment ion species from peptide backbone breakage

A- and $\mathrm{x}$-ions (blue) are generated after the breakage of the alkyl-carbonyl bond, the breakage of the amide (or peptide) bond results in b-and $y$-ions (green) and breakage of the amino-alkyl bond leads to $\mathrm{c}$ - and z-ions (orange). A-, b-, and c-ions contain the $\mathrm{N}$-terminal part of the peptide while $\mathrm{x}_{-}, \mathrm{y}-$, and $\mathrm{z}$ - ions the corresponding C-terminal part (Roepstorff and Fohlman, 1984).

Another common fragmentation technique is electron transfer dissociation (ETD) where the peptide ions collide with an electron donor. This reduces the peptide charge by one and generates an unpaired electron on the peptide. The peptide ions become instable and break between the $\mathrm{N}-\mathrm{C} \alpha$ bond, thereby generating $\mathrm{c}$ - and $\mathrm{z}$-fragment ions (Figure 1.3). ETD fragmentation is advantageous for very large peptides or peptides modified with a labile PTM as serine and threonine phosphorylation or O-linked GlcNac (Coon et al., 2005; Jedrychowski et al., 2011).

\subsubsection{Mass spectrometry instrumentation}

Modern tandem mass spectrometry is performed on instruments that possess two different mass analyzer devices, so called hybrid instruments. There are many types of mass analyzers available in hybrid instruments, most commonly: quadrupole, ion traps (cubic or linear ion trap), time-of-flight (TOF) and orbitraps. Every analyzer type offers different performance characteristics, such as mass accuracy, $m / z$ range, dynamic range, scan speed and resolving power. Moreover, different mass analyzers show different levels of suitability for functionalities such as ion separation, ion trapping and fragmentation or detection. Mass analyzers can be divided into high resolution and low resolution analyzers: orbitrap and TOF analyzers belong to the high resolution class whereas quadrupoles and linear ion traps belong to the low resolution analyzers. The advantages of different $\mathrm{m} / \mathrm{z}$ separating devices and analyzers are exploited by their combination in hybrid instruments (Glish and Burinsky, 2007). Common combinations are e.g. linear ion trap-orbitrap, quadrupole-TOF or quadrupole- 
orbitrap. Due to the huge contribution of orbitrap-based mass spectrometry for this research project, this technique will be explained in more detail.

The basic idea behind the orbitrap mass analyzer existed previously as the so-called ideal Kingdon trap' that was invented in its actual form by Alexander Makarov (Makarov, 2000). The working principle is similar to FT-ICR where the ion motion is controlled by an axial magnetic field (Gillig et al., 1996). The orbitrap mass analyzer does not require a magnetic but an electrostatic field. It consists of two parts, an axial central electrode and a two part outer electrode parallel to the axis of the central electrode. The electrostatic field traps ions rotating around and oscillating along its central electrode (Makarov, 2000). The frequencies of the oscillating axial movement are detected by an image current induced between the halves of the outer electrode and are converted by Fourier transformation into $m / z$ values (Scigelova and Makarov, 2006). The orbitrap mass analyzer is a high resolution $>70.000$ and sub-ppm high mass accuracy mass analyzer (Olsen et al., 2005). Recently, an advancement of the orbitrap analyzer was introduced, the high-field (HF) orbitrap. Due to the reduced trap size, increased field strength can be applied and therefore a higher resolution can be achieved within the same transient time (Makarov et al., 2009; Scheltema et al., 2014).

\subsubsection{Shotgun proteomics workflows}

A typical MS-based proteomics experiment can be divided into several parts: protein extraction, fractionation and/or enrichment on the protein or peptide level, proteolytic digestion, RP-HPLC separation, ESI followed by mass spectrometric analysis of the precursor and fragment ions, and database search (Figure 1.4). 


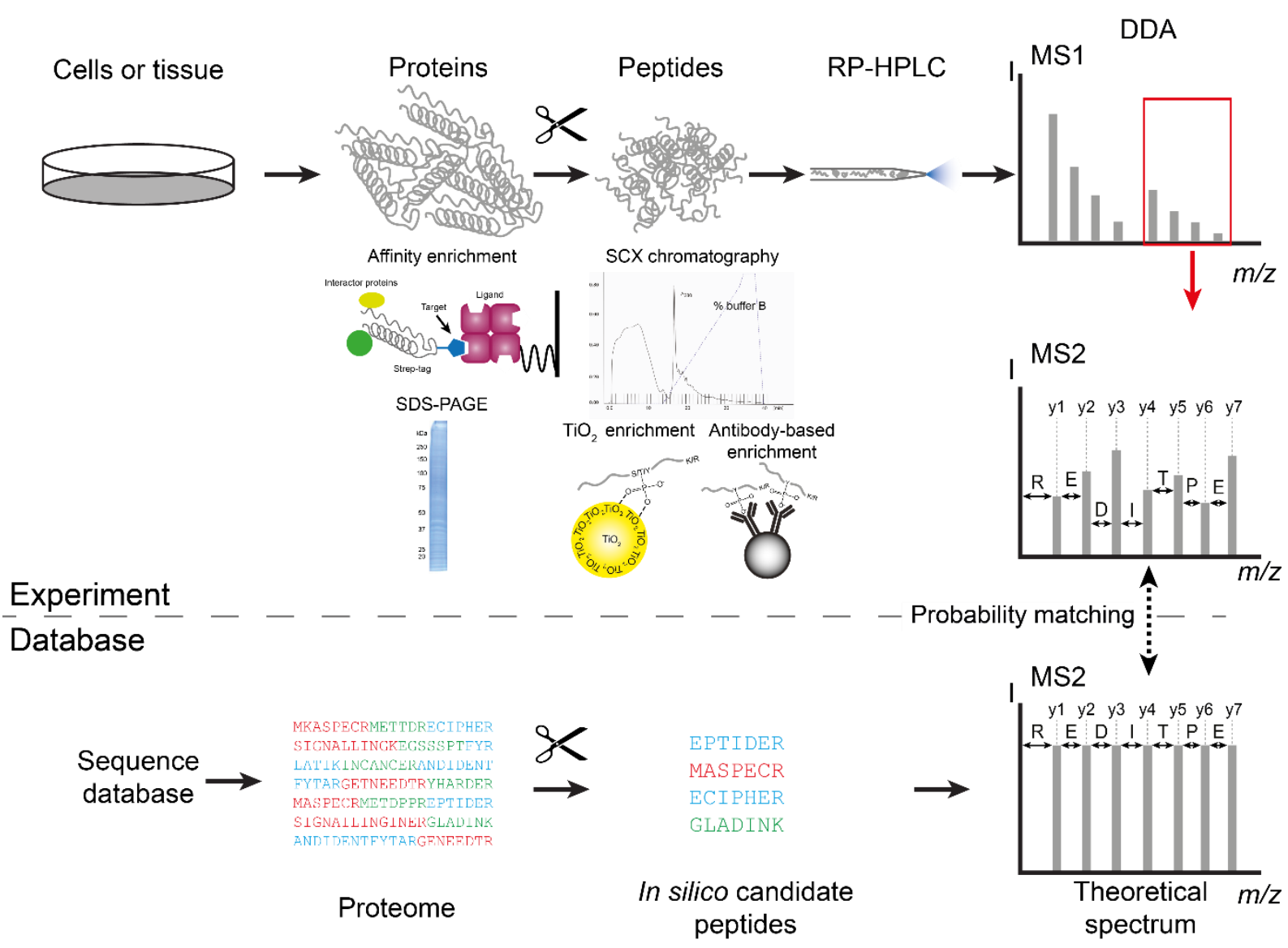

Figure 1.4: Outline of generic building blocks of a bottom-up/shotgun proteomics workflow

The starting material (cells or tissues) can be metabolically or chemically labeled. Samples can be subjected to fractionation or enrichment on the protein level or after digestion with the respective endoproteinase (represented by scissors) to peptides. Peptides are subjected to RP-HPLC, ionized by ESI and the charged ions are then transferred into the gas phase to the mass spectrometer. During the whole RP-HPLC gradient ions are transmitted to the mass spectrometer for DDA. A survey scan (MS1) is followed by a user defined number of consecutive fragment scans (MS2) of the highest abundant precursor ions. Peptides are identified by matching theoretical peptide fragmentation spectra derived from a sequence database after in silico digest to the experimentally acquired spectra. RP-HPLC: reversed-phase high performance liquid chromatography; DDA: data-dependent acquisition; SCX: strong cation exchange; $\mathrm{TiO}_{2}$ : Titanium dioxide; I: Intensity; $\mathrm{m} / \mathrm{z}$ : mass-to-charge ratio.

The proteome can be extracted from different sources such as cell culture or paraffinembedded tissue samples. It can be fractionated or specifically enriched for PTMs, specific proteins or classes by affinity- or immunopurification (Figure 1.4). A common fractionation step is SDS-PAGE where proteins are separated according to apparent molecular weight and interfering detergent or salt ions from cell culture or lysis buffers are removed. For protein interactome analysis affinity purification or antibody-based purification of the target protein is performed (Figure 1.4).

In bottom-up proteomics the intact proteins are hydrolyzed into smaller peptides with a sequence specific endoproteinase (Figure 1.4). This can be done either in-gel, after SDS-PAGE, or in solution (Shevchenko et al., 2007). Most commonly trypsin is the proteinase of choice 
because it cleaves with high specificity C-terminally of arginine (R) and lysine (K). The frequency of occurrence of arginine and lysine in the proteome is advantageous to generate peptides of an optimal MS/MS length (between 7 and 20 amino acids) (Olsen et al., 2004). Moreover, arginine and lysine have basic side chains and therefore can carry, besides the $\mathrm{N}$ terminal $\mathrm{NH}_{2}$-groups, a second proton after ionization. Other frequently used proteinases are Lys-C which cleaves C-terminal to lysine, or chymotrypsin which cleaves C-terminal of leucine and the aromatic amino acids phenylalanine, tryptophan and tyrosine, respectively (reviewed in Zhang et al., 2013b).

Fractionation or enrichment steps do not necessarily have to occur prior to digestion. Especially PTM enrichment steps are preferentially performed after in-solution digest on the peptide level to increase the binding selectivity and thereby increase the sensitivity of LCMS/MS detection (reviewed in Zhao and Jensen, 2009). Peptide fractionation is mostly based on ion exchange chromatography providing an orthogonal separation method compared to reversed phase chromatography. Enrichment procedures for PTMs involve affinity purification e.g. by metal affinity complexation for phosphorylated peptides, lectins for glycosylated peptides or immunoprecipitation with PTM-specific antibodies (Figure 1.4) (reviewed in Macek et al., 2008; Nilsson, 2011; Zhang et al., 2013a).

The resulting sample peptides are submitted to reversed-phase high performance liquid chromatography (Figure 1.4). The stationary phase is non-polar, therefore peptides are separated according to their hydrophobicity. The elution is achieved by an increase of the nonpolar solvent like acetonitrile or methanol together with an ion pairing reagent e.g. trifluoroacetic acid (TFA) or an organic acid such as formic acid (FA) in the mobile phase (reviewed in Mallick and Kuster, 2010). Throughout the whole RP-HPLC gradient, MS1 and MS2 spectra are consecutively recorded with a user-defined acquisition method. The acquisition method is most commonly DDA (Figure 1.4; previously described in section 1.3.1) and defines several parameters, i.e. selection of the top $\mathrm{N}$ most abundant precursor ions for fragmentation, use of a defined isolation window for selected precursor ions, fragmentation energy, MS1 and MS2 resolution, number of ions and the time required for measurement, exclusion of already sequenced ions. The resulting raw data is submitted to a search engine e.g. Andromeda or Mascot. Spectra interpretation occurs in an automated fashion by matching experimentally acquired spectra to theoretical spectra derived from a database such as UniProt (Figure 1.4). 


\subsubsection{Quantitation}

Besides the $m / z$ ratios of the precursor and the fragment ions, a third piece of information is acquired during mass spectrometric measurement, the peptide ion intensity. While the $m / z$ values identify a peptide/protein, its intensity (on the MS1 or the MS2 level, respectively) provides quantitative information. Overall, quantitation strategies can be divided into relative and absolute quantitation (Table 1). In relative quantitation, one or more experimental states are compared to a reference state, whereas in absolute quantitation the protein concentrations are determined (reviewed in Zhang et al., 2013b). Absolute quantitation can be achieve either in a label-free manner or by using a defined amount of a labeled standard (Bantscheff et al., 2007b; Schwanhäusser et al., 2011). For this work, only relative quantitation strategies were applied and therefore will be explained in more detail. Relative quantitation strategies can be achieved by labeling or label-free approaches. Labeling strategies can be divided in metabolic and chemical/enzymatic approaches. A common chemical labeling strategy is isobaric tags for relative and absolute quantitation (iTRAQ). The iTRAQ reagent reacts with the primary amine group of the N-terminus or lysine side chains. Quantitation is performed after fragmentation of the peptide on the MS2 level (Ross et al., 2004). The most popular and successful labeling strategies due to its robustness and accuracy is the stable incorporation of labeled amino acids in cell culture (SILAC) (Ong and Mann, 2007; Ong et al., 2002). Label-free quantitation is based on ion intensities or spectral counting approaches (reviewed in Nikolov et al., 2012). For this work, SILAC labeling and label-free quantitation were used and thus will be explained in more detail.

Table 1: Overview of commonly applied label-based and label-free relative and absolute quantitation strategies (Nikolov et al., 2012).

\begin{tabular}{|c|c|c|c|c|}
\hline \multicolumn{3}{|c|}{ Relative quantitation } & \multicolumn{2}{|c|}{ Absolute quantitation } \\
\hline & bel-based & Label-free & Label-free & Label-based \\
\hline Metabolic & Chemical/Enzymatic & & & \\
\hline SILAC & $\begin{array}{c}\text { iTRAQ } \\
\text { TMT } \\
\text { DML }\end{array}$ & $\begin{array}{c}\text { XIC-based } \\
\text { Spectral counting }\end{array}$ & AQUA peptides & iBAQ \\
\hline
\end{tabular}

SILAC: stable isotope labeling by amino acids in cell culture; iTRAQ: isobaric tags for relative and absolute quantitation; TMT: tandem mass tag; DML: dimethyl labeling; XIC: extracted ion chromatogram; AQUA: absolute quantitation; iBAQ: intensity based absolute quantitation. 


\subsubsection{Stable isotope labeled amino acids in cell culture (SILAC)}

The SILAC amino acids are introduced metabolically i.e. during the growth of the cells. Therefore, cells are cultivated in medium that is depleted of arginine (R) and lysine (K) and instead is supplemented with the respective labeled lysine and arginine amino acids (Ong et al., 2002). Cells have to undergo several rounds of cell divisions, to achieve full incorporation of the labeled amino acids (Ong and Mann, 2007). 'Heavy' or labeled amino acids contain different numbers of the stable isotopes ${ }^{2} \mathrm{H}$ (deuterium), ${ }^{13} \mathrm{C},{ }^{15} \mathrm{~N}$ or ${ }^{18} \mathrm{O}$ which introduces a mass shift in the MS1 spectrum. Generally, arginine and lysine are labeled because after tryptic digest peptides contain ideally one heavy C-terminal amino acid. SILAC experiments can be conducted as triplex experiments, comparing up to three different cell conditions states: light $(K+0, R+0)$, medium or intermediate $(K+4, R+6)$ and heavy $(K+8, R+10)$. The intensity differences of the fragments in the MS1 spectrum reflect the relative quantities of the different states. Due to early mixing of the samples, ideally after cell lysis, parallel sample handling makes the method robust and less prone to sample preparation bias (Figure 1.5) (Ong and Mann, 2006). However, not all biological systems are amenable to SILAC labeling e.g. in vivo probes or non-auxotrophic cell lines. For those, alternative quantitation strategies such as label-free quantitation can be applied.

\subsubsection{Label-free quantitation}

Label-free quantitation has evolved as a serious addition to the quantitation toolbox as with improved MS instrumentation, computational power and algorithms, quantitation value determination became more robust. Two label-free quantitation approaches can be distinguished: spectral counting or quantitation of peak area intensities (Table 1). Spectral counts are a measure of the number of MS/MS sequencing events that lead to peptide identification originating from the same protein during an LC-MS/MS run. The assumption is that the abundance of the proteins correlate with MS/MS spectrum matches (Liu et al., 2004; Washburn et al., 2001). Another approach is the quantitation of integrated peptide peak areas observed in extracted ion chromatograms (XICs), derived from the same protein (Cox et al., 2014b). The protein abundance ratio is measured by comparing the XIC intensities of same peptides of proteins from different experiments (Bondarenko et al., 2002).

Advances in label-free quantitation were made through improvements in instrumentation and quantitation algorithms. With fast acquisition, high mass resolution and accuracy MS instruments, more data points can be collected. Consequently, more sophisticated 
quantitation algorithms can be applied e.g. for normalization of data. Recently an advancement of XIC-based label-free quantitation was published, the MaxLFQ algorithm which is implemented in the MaxQuant computational proteomics platform which was used in this work (Cox et al., 2014; Cox and Mann, 2008). Label-free methods can be applied to samples that cannot be metabolically labeled and the number of samples that can be compared to each other is unlimited. However, label-free quantitation leads to an increase in measuring time due to parallelized measurements, the requirement for simple, robust sample handling and is more prone to potential inaccuracy (Figure 1.5) (Cox et al., 2014; Dephoure and Gygi, 2012).

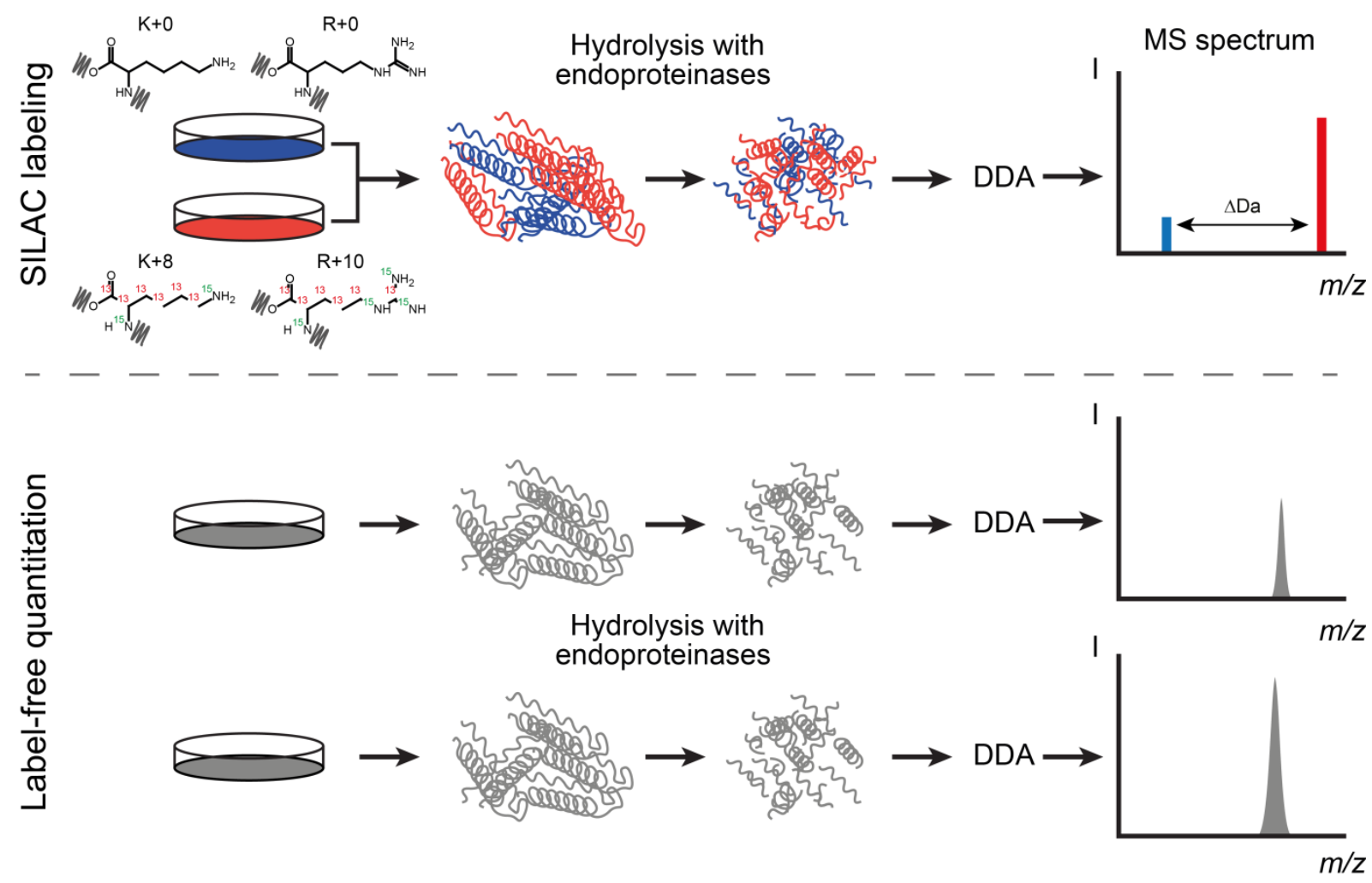

Figure 1.5: SILAC labeling and label-free quantitation

The upper panel shows a schematic double SILAC labeling workflow. The light $(K+0, R+0)$ and heavy $(K+8, R+10)$ amino acids are introduced during cell growth. The isotope positions in the amino acids are indicated $\left({ }^{13} \mathrm{C}:\right.$ red, ${ }^{15} \mathrm{~N}$ : green). Cell lysates are mixed, digested and measured by mass spectrometry. Quantitation is achieved by comparing MS1 intensities of the different SILAC states. The lower panel shows a label-free quantitation approach comparing two different states. Sample preparation and measurement are performed in parallel. Quantitation is achieved by comparing the XICs of the two different states. SILAC: stable isotope labeling by amino acids in cell culture; K: lysine; R: arginine; $\mathrm{m} / \mathrm{z}$ : mass-to-charge ratio; DDA: data-dependent acquisition.

\subsubsection{Peptide and protein identification}

With the advent of high-throughput proteomics experiments, manual spectra interpretation became outdated and unfeasible. Hence, data produced from large-scale proteomic analyses 
require computational power, sophisticated search engines and highly complex data analysis algorithms to perform peptide and protein identification, PTM localization and quantitation with high confidence and in an automated fashion (reviewed in Bantscheff et al., 2012; Mallick and Kuster, 2010; Mann et al., 2002). Some search engines are stand-alone software such as Mascot or SEQUEST, whereas the search engine Andromeda is integrated in the proteomic software platform MaxQuant which offers a higher degree of data compiling and organization (Cox and Mann, 2008; Cox et al., 2011). Results derived from Mascot can be further integrated into platforms such as Proteome Discoverer, Skyline, Scaffold, Progenesis or OpenMS (Brownridge et al., 2012; Sturm et al., 2008) (reviewed in Zhang et al., 2013a). Every software platform offers unique advantages over the others and therefore different applications might require the use of several different platforms. The basic functionality of search engine identification is similar: theoretical MS2 fragment spectra are compared to experimentally acquired MS2 spectra. The pool of theoretical MS2 spectra relies on a database containing a defined set of protein sequence entries. Theoretical MS2 spectra are generated in silico according to endoproteinase specificity and, if applicable, quantitation labels and PTMs. Candidate MS2 spectra within the predefined MS1 precursor and MS2 fragment mass tolerances are matched to the experimentally obtained MS2 spectra. For every experimental spectrum several theoretical candidate peptides can be obtained. The identification is based on a score value which reflects the quality of a peptide-spectrum-match (PSM) between a theoretical and an experimental spectrum (reviewed in Zhang et al., 2013). To increase confidence on a global level, identifications are filtered according to their false discovery rate (FDR). The FDR is a statistical approach to estimate and control the number of false positive identified proteins. The FDR is estimated by matching the experimental data against a targetdecoy database, containing the forward and the reversed or scrambled protein sequences derived from the forward database. All entries in this 'nonsense' database are false hits by definition (Elias and Gygi, 2007). The target-decoy approach is used to estimate and control the FDR. Generally, a FDR of $1 \%$ is an accepted value for large data-sets.

For this work, the freely available computational platform MaxQuant with its search engine Andromeda was used due to its superior performance in large-scale, quantitative (SILAC and label-free) PTM analysis (Cox and Mann, 2008; Cox et al., 2011). MaxQuant performs SILACbased peptide, protein and PTM site quantitation by 3D peak extraction (retention time, $\mathrm{m} / \mathrm{z}$ value, intensity) on the MS1 level while label-free quantitation is based on XIC extraction as 
mentioned previously in section 1.3.6.2 (Cox et al., 2014). Quantitation values are calculated according to the user-specified experimental design can be accessed by plain, tab-separated text files with dedicated software tools to perform statistical and partly functional analyses.

\subsubsection{Identification of post-translational modification by mass spectrometry}

Previously, a large effort was made for high-throughput transcriptome and proteome analyses. However, transcriptome and proteome levels of enzymes might not correlate with the biological activity of a protein. Moreover, gene expression and protein copy number changes are far downstream processes that are initiated by an extracellular stimulus and therefore represent a final outcome rather than an active signal (reviewed in Larsen et al., 2006; Mann and Jensen, 2003). Post-translational modifications introduce an additional level of protein regulation by occurring immediately after and proximal to the initiating stimulus. They control many cellular processes as proliferation, metabolism, differentiation and apoptosis by changing the conformational state of a protein, tagging them for processing or subcellular relocalization or providing a binding platform and thereby regulating protein activity or degradation (reviewed in Mann and Jensen, 2003; Olsen and Mann, 2013; Witze et al., 2007). PTMs are covalent, chemical modifications of proteins after translation. Hundreds of different PTMs have been described, however only a handful of them are being investigated extensively: phosphorylation of serine, threonine and tyrosine, acetylation, ubiquitination, methylation, SUMOylation and $\mathrm{N}$ - and O-linked glycosylation (Jensen et al., 2006; Uy and Wold, 1977; Walsh et al., 2005). Figure 1.6 shows the PTMs that were investigated in this work. 
Phospho-serine<smiles></smiles>

Acetyl-lysine<smiles>CNC(CCCCNC(C)=O)C(=O)O[Na]</smiles>

Phospho-threonine<smiles>CC(OP(=O)([O-])[O-])C([NH3+])C(=O)O[Na]</smiles>

Phospho-tyrosine

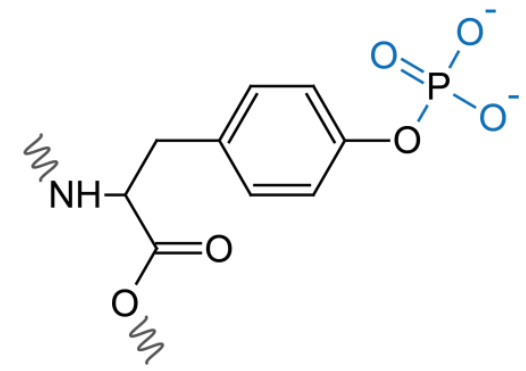

Lysine ubiquitination

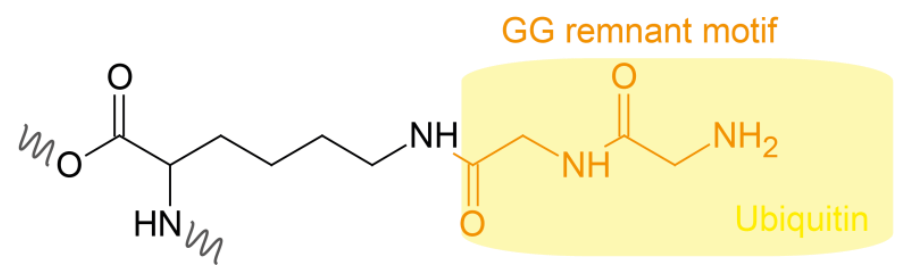

Figure 1.6: Post-translational modifications investigated in this work

The upper panel shows phosphorylated (highlighted in blue) serine, threonine and tyrosine side chains. The lower panel shows acetylated (red) and ubiquitinated (a generic ubiquitin molecule is depicted as yellow rectangle; the GG remnant motif is highlighted in orange) lysine side chains, respectively. The zigzag line indicates the peptide bond.

PTM-mediated signaling networks consist of a dynamic interplay between writer (e.g. tyrosine kinases), eraser (e.g. tyrosine phosphatases) and reader (e.g. SH2-domain containing proteins) enzymes that process information in a spatiotemporal manner such as the afore mentioned BCR and TCR signaling cascades after antigen encounter (reviewed in Jin and Pawson, 2012). A specific function is rarely mediated by just a single type or site of modification, but especially cross talk of different modifications leads to a precise cellular outcome (Hunter, 2007; Swaney et al., 2013). Cross talk entails combinatorial effects of PTMs and can be either negative or positive. Negative cross talk occurs in a competitive manner i.e. one PTM occupies the same position on the same amino acid side chain, e.g. acetylation and ubiquitination on lysine residues, or indirectly competitive by masking secondary PTM sites (Hunter, 2007; Wagner et al., 2011). Positive, non-competitive cross talk between PTMs is characterized by one PTM priming the addition or removal of a second or more PTMs leading to combinatorial regulation effects. Cross talk was first described in histones where a multitude of PTMs ('histone marks') on the N-terminal tail form the so-called histone code (Strahl and Allis, 2000). Another important example are phosphodegrons where an initial phosphorylation of a dedicated site on a protein is followed by subsequent ubiquitination leading to its proteasomal degradation (Ang and Harper, 2005). In general, PTMs can influence each other in many ways: (i) a first 
PTM regulates the catalytic activity of a second PTM writer or eraser enzyme by allosteric changes, (ii) a PTM provides a binding platform for a writer or eraser enzyme which then can mediate its catalytic activity on a site in close proximity or (iii) a PTM on a substrate hinders the interaction with the catalytic active enzyme by steric hindrance (Hunter, 2007).

As mentioned previously, the presence of a PTM on a peptide is accompanied by a mass increase of the precursor and, in most cases the sequence-specific fragment ions. From these, the position of the PTM on the peptide sequence can be determined (reviewed in Mann and Jensen, 2003). However, PTM identification is not a trivial task as some challenges have to be overcome. Recent developments in MS technology, sample preparation methods and computation made the generation of large-scale, high-throughput PTM datasets feasible (reviewed in Mann and Jensen, 2003; Olsen and Mann, 2013). One challenge that has to be dealt with is that all PTMs occur at substochiometric levels, meaning that only a small proportion of proteins are modified at any given time point. This leads to masking of the posttranslationally modified peptides by their unmodified, high abundant counterparts. Some PTMs can be enriched by making use of the physical and chemical properties of the PTM by affinity-based enrichment procedures or by their immunogenicity using target-specific antibodies (reviewed in Mann and Jensen, 2003; Witze et al., 2007). Not only specific enrichment steps are required, also the PTM eraser enzymes e.g. PSP/PTP, KDACs, DUBs need to be inhibited. Another challenge is that PTM identification requires high sequence coverage because every single PTM site stands for its own and can have an individual biological meaning. Also, efficient fragmentation is required in order to unambiguously localize the site of modification in case there are several theoretical possibilities (reviewed in Olsen and Mann, 2013). However, complete fragment ion series and unambiguous site localization cannot always be achieved for peptides with multiple potential sites of modification e.g. a phosphorylated peptides containing several serine, threonine or tyrosine residues, especially when potential acceptor residues are in close proximity to each other. The confidence of these positional PTM isomers is reflected by site localization scores such as the A score (implemented in SEQUEST), localization probability (implemented in Andromeda), Mascot Delta (MD) score and phosphoRS (embedded in Proteome Discoverer) (Beausoleil et al., 2006; Olsen et al., 2006; Savitski et al., 2010; Sharma et al., 2014; Taus et al., 2011). Of note, all of the above scoring schemes were originally developed for large-scale phosphorylation site analyses but can also be applied to other post-translational modifications. In the following the 
PTMs investigated in this work will be described in more detail in terms of biological function, enrichment strategies and mass spectrometric analysis.

\subsubsection{Phosphorylation}

Reversible serine, threonine and tyrosine phosphorylation is by far the most studied PTM (Figure 1.6). This can be partly explained by its early detection: Serine and threonine kinase activity was detected in the middle of the 50s, and tyrosine phosphorylation in the beginning of the 80 s of the last century (Burnett and Kennedy, 1954; Eckhart et al., 1979; Sefton et al., 1980). Also its major regulatory importance e.g. in immune receptor signaling and the availability of convenient enrichment procedure strategies have been important factors (reviewed in Hunter, 2007). Phosphorylation of a target protein leads commonly to changes in protein activity, protein-protein interactions or re-localization of proteins within a cell as described previously (reviewed in Hunter, 2007; Lim and Pawson, 2010). The proportions of modified amino acid residues reported in large-scale studies vary in the literature, dependent on the methodology used. However, serine is by far the most frequently phosphorylated amino acid (59\%) followed by threonine $(24.7 \%)$ and tyrosine $(16.3 \%$; according to PSP phosphorylation modification site statistics in September 2015, www.phosphosite.org). The addition of a phosphate group to a target amino acid increases the peptide or fragment mass by $80 \mathrm{Da}$ (reviewed in Larsen et al., 2006).

Additionally, the phosphorylation of the basic amino acids, lysine, arginine and histidine and cysteine residues has been described (Bertran-Vicente et al., 2014; Elsholz et al., 2012; Hess et al., 1988; Meins et al., 1993). The phosphorylation on these unconventional amino acids was for a long time believed to be merely found in prokaryotes. However, there is growing evidence that they could also play a role in eukaryotes (reviewed in Cieśla et al., 2011; Cozzone, 1988). The phosphorylation is rare compared to serine, threonine and tyrosine phosphorylation and is furthermore acid labile which makes common enrichment procedures unfeasible, therefore it will not be mentioned further in this thesis (Cieśla et al., 2011; Matthews, 1995).

Phosphorylation of serine, threonine and tyrosine is regulated by the dynamic interplay of kinases which add the $y$-phosphoryl group from the donor ATP to the amino acid residue, and phosphatases that remove the phosphate group (Hunter, 1995). 478 human eukaryotic protein kinases were identified that can be divided into seven major branches (CMGC, CAMK, AGC, CK1, STE, TKL and TK) and 40 atypical kinases (Manning et al., 2002). Atypical kinases do 
not have sequence homology to other kinases but execute kinase activity. Six branches of the eukaryotic protein kinases account for serine/threonine kinases while the tyrosine kinase (TK) family consists of 90 members of whom 58 are receptor tyrosine kinases (Lemmon and Schlessinger, 2010; Manning et al., 2002). Receptor tyrosine kinases mediate ligand-induced tyrosine phosphorylation for transmission of signals across a plasma membrane e.g. the EGFR or the insulin receptor family. Moreover, there are two-component receptors like the BCR and TCR antigen receptors that are composed of an extracellular ligand-binding subunit that forms a complex with a cytoplasmic tyrosine kinase (Samelson, 2011). Phosphorylated tyrosine residues are especially low abundant but are of major significance especially in the context of (immune)-receptor signaling. Tyrosine phosphorylation has slightly distinct chemical properties compared to its phosphorylated serine and threonine counterparts which provide binding specificity (reviewed in Hunter, 2014; Lim and Pawson, 2010). The phosphorylation is linked to the phenolic ring which makes hydrophobic or $\pi$-bond-ring interactions possible, and the phosphorylation is farther away from the peptide backbone which requires deeper binding pockets (reviewed in Hunter, 2014). These steric differences makes the generation of highlyspecific, motif-independent anti-phosphotyrosine antibodies possible which are a valuable tool for the investigation of tyrosine phosphorylation (Larsen et al., 2005; Rush et al., 2004). Phosphorylation is counteracted by the removal of the phosphate group by protein phosphatases which as kinases can be divided in two classes: protein serine/threonine phosphatases and protein tyrosine phosphatases (Hunter, 1995). Originally, phosphorylated serine and threonine residues were thought to merely introduce allosteric changes in proteins rather than providing protein-protein interaction platforms (reviewed in Hunter, 2014). However, five domain families that bind phosphoserine and threonine were identified that regulate various mechanisms like ubiquitination, cell cycle control and DNA damage signaling (Yaffe and Elia, 2001). Specific phosphotyrosine-binding domains comprise of the Src homology 2 (SH2) and the phosphotyrosine-binding (PTB) domain and can be primarily found in adapter proteins proximal of immune-receptor such as SYK or ZAP-70, BTK, GRB2, NCK and many others (reviewed in Schlessinger and Lemmon, 2003; Yaffe, 2002).

Standard enrichment strategies for phosphorylated peptides include the complexing of the phosphate group with immobilized metals e.g. IMAC or titanium/zirconium metal oxides $\left(\mathrm{TiO}_{2}\right.$ or $\mathrm{ZrO}_{2}$ ). This affinity complexation exploits the chemical behavior of the phosphate group regardless of the site of modification (reviewed in Witze et al., 2007). Additionally, 
phosphotyrosine can be specifically enriched with the respective antibodies whereas phosphoserine and -threonine specific antibodies are not available (reviewed in Grimsrud et al., 2010).

Under CID fragmentation, phosphorylated serine and threonine residues are prone to neutral loss of phosphoric acid $\left(\mathrm{H}_{3} \mathrm{PO}_{3}, 98 \mathrm{kDa}\right)$ (Boersema et al., 2009; Schroeder et al., 2004). Tyrosine residues tend to lose metaphosphoric acid $\left(\mathrm{HPO}_{3}, 80 \mathrm{kDa}\right.$ ) (reviewed in Mann et al., 2002; Mann and Jensen, 2003). This leads to inefficient fragmentation of the peptide backbone and requires an additional fragmentation step (MSn or MS3). Furthermore, tyrosine residues tend to $\mathrm{N}$ - and $\mathrm{C}$-terminal fragmentation that lead to a characteristic diagnostic immonium ion of $\mathrm{m} / \mathrm{z} 216.0426$ (Steen et al., 2001). As mentioned before, the low mass cutoff of ion traps prevents the low mass tyrosine immonium ion from detection in many cases. In beam-type CID (HCD) and ETD fragmentation the phosphorylation detaches to a lesser extend from the peptide due to different fragmentation principles and mechanisms. Phosphorylated amino acids stay intact and the peptide backbone gets fragmented which leads to more meaningful spectra (Nagaraj et al., 2010). The advent of different fragmentation options posed a challenge to the community to find the optimal conditions for phosphorylated peptides. In several studies it was shown that beam-type CID (HCD) outperforms CID for the fragmentation of phosphopeptides (Frese et al., 2011; Marx et al., 2013; Nagaraj et al., 2010; Savitski et al., 2010). Furthermore it was shown that HCD in general gives a better outcome for the identification of phosphopeptides compared to ETD, although the latter is more suitable for higher charge states and leads to more unambiguous site identification (Marx et al., 2013). Current efforts aim at the development of alternative fragmentation techniques for particularly fragile modifications. Recently a hybrid fragmentation mode that combines ETD and HCD fragmentation, namely EThcD, that is especially suitable for phosphorylation site localization was described (Frese et al., 2012; 2013).

\subsubsection{Deregulated phosphorylation}

Phosphorylation is undoubtedly of major importance in cell signaling, and its deregulation caused by dysfunctional kinases has been already associated with several types of diseases e.g. Alzheimer's disease, immunological diseases, metabolic diseases and especially cancer (Bramblett et al., 1993; Goedert, 2015) (reviewed in Cohen, 2002). Kinases with an oncogenic potential regulate important signaling pathways at central positions. These kinases can be either directly affected by mutation or they act as downstream recipients of oncogenic 
upstream signals (reviewed in Zhang et al., 2008). Direct deregulation which render the kinases constitutively active can be caused by various mechanisms, amongst others by an internal tandem duplication e.g. of the FLT3 receptor in AML (FLT3-ITD), amino acid substitution e.g. BRAF V600E which affects the kinase activation loop or gene fusions e.g. BCRABL1 (also called Philadelphia chromosome) (Davies et al., 2002; Quentmeier et al., 2003) (reviewed in Zhang et al., 2008a). A kinase affected by a mutational hit withdraws itself from regulation and therefore cell survival becomes dependent on this kinase, a process referred to as oncogene addiction. This dependency also makes the malignant cell vulnerable to kinase ablation (Weinstein et al., 1999; Weinstein and Joe, 2006). Given the importance of kinases for cell signaling, they are an important target for pharmacological manipulation. Kinases have a bi-lobed structure, with an active-site cleft between the $\mathrm{N}$ - and C-terminal lobe and a highly conserved activation loop that lies outside the cleft. The ATP interaction occurs in the catalytic cleft within the hinge that connects both lobes. Kinase activity itself is regulated by phosphorylation of amino acid residues in the activation loop leading to conformational changes, for example MAP-kinases possesses a TXY motif in their activation loop that is required to be phosphorylated at both positions to become fully active (Burack and Sturgill, 1997; Canagarajah et al., 1997). At the $\mathrm{N}$-terminal side of the activation loop protein kinases possess an aspartate-phenylalanine-glycine (DFG) motif that mediates catalytic activity. In the active, phosphorylated conformation (DFG in), the loop can interact with the substrate whereas in the inactive kinase conformation (DFG out), the activation loop blocks the substrate binding site (Nagar et al., 2002). The common ATP dependency of phosphorylation makes the ATP interaction sites a popular target for inhibitor design. The ATP binding sites of kinases are highly conserved, yet sufficiently distinct to achieve a certain degree of specificity to fit in chemical compounds. These are small molecules that function as ATP analogues and therefore as selective kinase inhibitors (Capdeville et al., 2002) (reviewed in Zhang et al., 2008). The majority of small-molecule kinase inhibitors are ATP competitors that target the kinase active site. Staurosporine, a microbial alkaloid produced by the bacterium Streptomyces staurosporeus was detected early as a kinase inhibitor but exhibited low specificity (Sako et al., 1988). In 1986, Novartis started with the development of a specific Abelson tyrosine kinase (ABL1) inhibitor which was 15 years later approved for clinical use in chronic myelogenous leukemia, named Imatinib or Gleevec ${ }^{\circledR}$ (Capdeville et al., 2002). Since then, several small-molecule kinase inhibitors have been approved for clinical administration 
for diverse indications and many more are currently in clinical studies (Cohen and Alessi, 2012). Two types of ATP-competitive kinase inhibitors can be classified: Type I inhibitors bind to the kinase in a conformation-independent manner (e.g. Dasatinib), while type II inhibitors only bind in the inactive conformation (e.g. Imatinib) (Hari et al., 2013; Schindler et al., 2000). Most small-molecule inhibitors bind reversibly, however, there are also irreversible inhibitors that form a covalent bond with a cysteine or nucleophilic residue in the kinase active site e.g. the BTK inhibitor Ibrutinib (Cohen et al., 2005). Another class are allosteric inhibitors that bind outside the ATP-binding site thereby inducing activity altering allosteric changes e.g. the MEK1 and MEK2 inhibitor $\mathrm{Cl}-1040$ (Ohren et al., 2004). Targeted therapies lead to improvements in cancer treatment but are hampered by resistance mechanisms against kinase inhibitors due to the selective pressure for cells. These resistance mechanisms can be acquired by mutations in the kinase, target amplification, upregulation of alternative pathways that drive cancer (reviewed in Zhang et al., 2008). The class of selective ATP competitive kinase inhibitors has grown and the search for new inhibitors and potential new targets is still going on.

\subsubsection{Acetylation}

Another important PTM is the acetylation of proteins on lysine residues. For a long time acetylation was mostly known in the context of epigenetic marks at the $\mathrm{N}$-terminal histone tail, thereby regulating gene transcriptional activity (Allfrey et al., 1964). Transcriptionally active euchromatin is associated with acetylation while inactive heterochromatin is modified to a lesser extent. Later, acetylation was also recognized to modify non-histone proteins such as tubulin and p53 (Gu and Roeder, 1997; L'Hernault and Rosenbaum, 1985). Acetylation is nowadays recognized as the predominant PTM in mitochondria and is associated with the regulation of metabolic processes (Kim et al., 2006; Wang et al., 2010; Weinert et al., 2011; Zhao et al., 2010). The acetyl group is transferred from acetyl-coenyzme A (acetyl-CoA) to the $\varepsilon$-amino group of lysine residues by the class of acetyltransferases (KATs), and can be removed by lysine deacetylases (KDACs) (Figure 1.6). In contrast, the $\mathrm{N}$-terminal protein acetylation is a common co-translational modification in eukaryotes that differs from PTM acetylation and will not be further discussed here (Hwang et al., 2010; Polevoda and Sherman, 2000). Bromodomains are the acetyl-lysine reader domains and can be mainly found in lysine acetyltransferases and nuclear proteins (reviewed in Zeng and Zhou, 2002). Recently, bromodomain inhibitors evolved as new class of small-molecule inhibitors that can be applied for the targeted inhibition of oncogene transcription (Delmore et al., 2011). The generation of 
acetyl-Iysine-specific antibodies made the efficient enrichment in a large-scale, MS-based manner possible (Choudhary et al., 2009; Kim et al., 2006). Acetylation induces as mass shift of $42 \mathrm{Da}$ and can be confused with trimethylation which has the same nominal mass. As for ubiquitination, trypsin does not cleave after acetylated lysine, formally leading to a missed cleavage (reviewed in Zhang et al., 2013).

\subsubsection{Ubiquitination}

Ubiquitin (Ub) is a 76 amino acid long protein which is attached with its C-terminal glycine to the lysine $\varepsilon$-amino group of a substrate protein (Figure 1.6 shows the di-glycine remnant motif after trypsin digestion). Ubiquitination is a three step process starting with ATP-dependent ubiquitin activation by an ubiquitin-activating enzyme (E1), followed by the transfer to an ubiquitin-conjugation enzyme (E2) and finally the transfer to the target protein by an ubiquitin-protein ligase (E3) (Ye and Rape, 2009). The modification can be reversed by deubiquitinating enzymes (DUBs). A single Ub-molecule or poly-Ub chains can be added to a target protein. For the assembly of poly-Ub chains the seven lysine residues in ubiquitin, and additionally the $\mathrm{N}$-terminal methionine (Met1) can become ubiquitinated and lead to different branched structures (Fiil and Gyrd-Hansen, 2014). Poly-Ub chains iteratively linked to lysine residue 48 (K48) mark proteins for proteasomal degradation (Fiil and Gyrd-Hansen, 2014; Kulathu and Komander, 2012; Thrower et al., 2000). In contrast, K63 linked poly-Ub does not drive degradation but is involved in various cellular regulation and signaling processes (Kulathu and Komander, 2012; Sun and Chen, 2004). Linear methionine ubiquitination (Met1$\mathrm{Ub}$ ) is linked to NF-KB signaling regulation whereas the other possible poly-Ub chain variants and mono-Ub regulate degradation or different cellular signaling mechanisms, respectively (Iwai and Tokunaga, 2009; Kulathu and Komander, 2012). Eleven families of Ub-binding domains (UBDs) are known that either bind to single ubiquitination or to different linked variants of the poly-Ub chains (Hicke et al., 2005) (reviewed in Kirkpatrick et al., 2005).

Ubiquitin enrichment for mass spectrometric measurement can be achieved by the expression of His-tagged ubiquitin in combination with anti-His purification protocols or by using ubiquitin binding domains for affinity purification (Hicke et al., 2005; Kirkpatrick et al., 2005; Peng et al., 2003). Furthermore, the intact ubiquitin molecule can be enriched with an antiubiquitin antibody (Vasilescu et al., 2005). Recently, a K-GG remnant motif antibody that proved to be a valuable tool for the large-scale enrichment of ubiquitinated peptides was developed (Figure 1.6) (Xu et al., 2010). As mentioned before, ubiquitin is attached with its C- 
terminal glycine at position 76 to the lysine side chain of the target protein (Figure 1.6). During digestion with trypsin, ubiquitin is cleaved between the arginine at position 74 and glycine at position 75. The motif is immunogenic and therefore can be targeted by specific antibodies. The C-terminal end of the ubiquitinated lysine cannot be cleaved by trypsin leading to a missed cleavage. However, after digestion neither mono- or poly-ubiquitination nor the linkage structure of poly-ubiquitination can be deduced anymore (reviewed in Kirkpatrick et al., 2005b; 2005a). The missed cleavage and the remnant motif are characteristic for MS-based identification of ubiquitin as the latter introduces a mass shift of 114.0429 Da on the respective lysine residue. The identification of ubiquitin remnants has to be considered carefully as the use of iodoacetamide for cysteine alkylation during sample preparation can introduce artifacts that mimic the GG remnant (Nielsen et al., 2008). Recently it was shown that ETD is the fragmentation method of choice for the identification of ubiquitinated peptides as those peptides are higher charged on average due to the missed cleavage (trypsin does not cleave after modified lysine) and the additional $\mathrm{N}$-terminus of the di-glycine adduct (PorrasYakushi et al., 2015). 


\subsection{Objectives}

Post-translational protein modifications such as serine, threonine and tyrosine phosphorylation play a crucial role in translating extracellular signals into highly specific cellular responses by a complex interplay of intracellular signaling cascades. Previously such signaling cascades have been investigated by low-throughput and targeted approaches. As dysregulated signaling is often involved in diseases such as cancer, global knowledge about phosphorylation dynamics is required to understand transformed signaling processes of malignant cell and to identify potential pharmacological targets such as kinases. Tandem mass-spectrometry is a suitable tool for the global and unbiased detection, quantitation, and localization of phosphorylation sites on proteins in a given cellular context. Like most PTMs phosphorylation occurs at substoichiometric levels, and therefore phosphopeptides need to be enriched by selective enrichment strategies prior to MS analysis. In combination with SILAC labeling, different cellular states can be compared simultaneously in one experiment. This work comprises three distinct large-scale, quantitative phosphoproteomic projects. The main project of this work is the investigation of B-cell receptor (BCR) signaling in Burkitt's lymphoma (BL), an aggressive B-cell derived lymphoma. Currently, BL is treated with aggressive chemotherapy as targeted therapeutic options are not available. BL cells critically depend on $B C R$ expression and BL cell survival is thought to be driven by antigen-independent, so-called tonic BCR signaling. Until now tonic BCR signaling has not been analyzed and it is not known if tonic and antigen-dependent BCR signaling activate similar effector proteins. To characterize these two modes of receptor signaling, antigen-dependent signaling was analyzed by timedependent receptor stimulation, whereas tonic BCR signaling was investigated upon CD79A knockdown and pharmacological SYK inhibition, respectively. Antigen-dependent phosphoproteomic analyses were complemented with transcriptional analysis and the determination of activated kinases to further characterize signaling mechanisms, and to identify other potentially pharmacologically targetable effectors.

Quantitative phosphoproteomics was applied to analyze another hematological disease, namely acute myeloid leukemia ( $\mathrm{AML}$ ). $\mathrm{AML}$ is an aggressive, hematologic cancer whose outcomes depend on the genetic background of malignant transformed cells. BTK, a tyrosine kinase known to be involved in BCR signaling was found to be activated in AML and its inhibition was shown to induce apoptosis in AML cells in preclinical models. Two genetically different $A M L$ cell lines were investigated on their phosphoproteomic level to investigate the 
activation mechanism of BTK and signaling alterations upon BTK inhibition to generate a molecular rationale for BTK inhibitors in AML treatment. T cell signaling is similar to that of $B$ cells although the immunological effector functions of B and T cells differ. Phosphorylation site dynamics of two essential TCR signaling adapters, LCP2 (SLP76) and GADS (GRAP2), were analyzed in a defined TCR stimulation time course in Jurkat cells to better understand their modes of action and regulation. Taken together, the aim of the presented work is to globally characterize phosphorylation site changes in different cellular conditions by quantitative mass spectrometry-based phosphoproteomics, with a particular focus on identifying regulatory $p$ sites on effector proteins that can be further investigated functionally and that might point towards druggable protein targets. 


\section{Materials and Methods}

\subsection{Materials}

\subsubsection{Technical devices}

Table 2: Technical devices

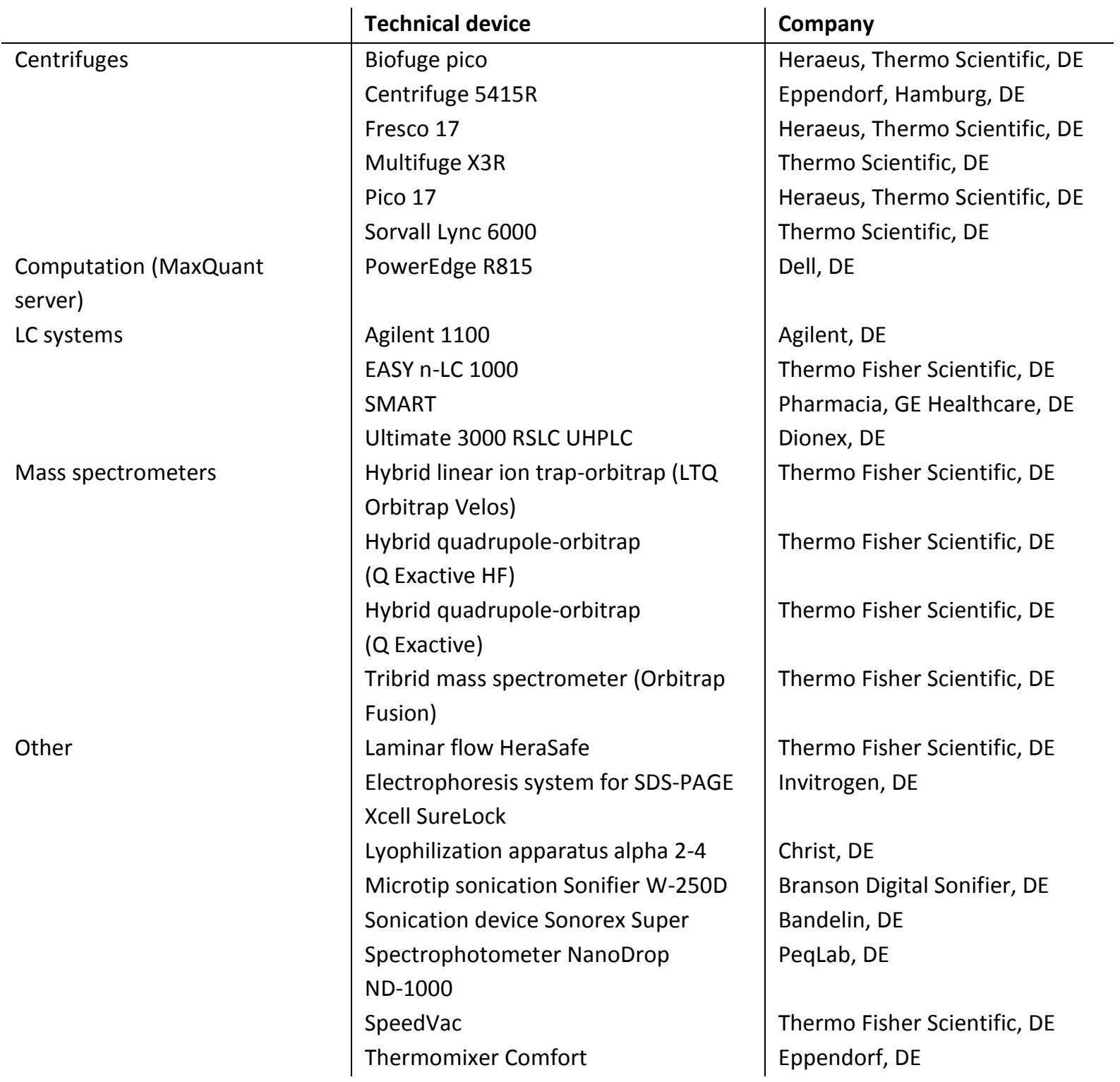

\subsubsection{Chemicals and reagents}

Table 3: Chemicals and reagents

\begin{tabular}{l|l} 
Chemicals and reagents & Company \\
\hline 2,2'-(Propane-1,3-diyldiimino)bis[2-(hydroxymethyl)propane-1,3-diol (Bis- & Sigma-Aldrich, DE \\
tris propane) &
\end{tabular}


Acetone

Acetonitrile (ACN), LiChroSolv

Ammonium bicarbonate (ABC)

Ammonium formate

Ammonium hydroxide $\left(\mathrm{NH}_{4} \mathrm{H}_{2} \mathrm{O}\right)$ 28-30\% (w/v)

C18 column material: ReproSil-Pur 120 C18-AQ, $120 \AA ̊(1.9 \mu \mathrm{m}, 3 \mu \mathrm{m}, 5 \mu \mathrm{m})$

C18 Empore Octadecyl $47 \mathrm{~mm}$ Extraction Disks

Chloroacetamide (CAA)

Coomasie Brilliant Blue G-250

Dihydroxybenzoic acid (DHB)

Dithiothreitol (DTT)

Ethanol, LiChroSolv

Formic acid (FA)

Glycerol, >99\%, p.a.

HEPES

IAP buffer (10x)

lodoacetamide (IAA)

Methanol, LiChroSolv

Methylated ethanol

NuPAGE Antioxidant

NuPAGE LDS sample buffer (4X)

NuPAGE MOPS SDS running buffer (10X)

NuPAGE Sample reducing agent (10X)

Potassium phosphate dibasic $\left(\mathrm{K}_{2} \mathrm{HPO}_{4}\right)$

Potassium phosphate monobasic $\left(\mathrm{KH}_{2} \mathrm{PO}_{4}\right)$

RapiGest

Sodium dodecyl sulphate (SDS)

Titanium dioxide $\left(\mathrm{TiO}_{2}\right)$

Trifluoroacetic acid (TFA)

Tris(2-carboxyethyl)phosphin (TCEP)

Tris(hydroxymethyl)aminomethane (Tris)

Urea

Water, LiChroSolv
Merck, DE

Merck, DE

Sigma-Aldrich, DE

Sigma-Aldrich, DE

Acros Organics, USA

Dr. Maisch, DE

3M, USA

Sigma-Aldrich, DE

Sigma-Aldrich, DE

Sigma-Aldrich, DE

Roth, DE

Merck, DE

Fluka and Sigma-Aldrich, DE

Roth, DE

Merck, DE

Cell Signaling Technology, DE

Sigma-Aldrich, DE

Merck, DE

Merck, DE

Invitrogen, DE

Invitrogen, $\mathrm{DE}$

Invitrogen, DE

Invitrogen, DE

Merck, DE

Merck, DE

Waters, UK

Biorad, DE

GL Sciences, JP

Roth, DE

Sigma-Aldrich, DE

VWR, DE

Sigma-Aldrich, DE

Merck, DE

\subsubsection{Buffer and solutions}

All buffers and solutions were sterilized with bottle top or syringe $0.2 \mu \mathrm{M}$ sterile filters.

Table 4: Buffers and solutions

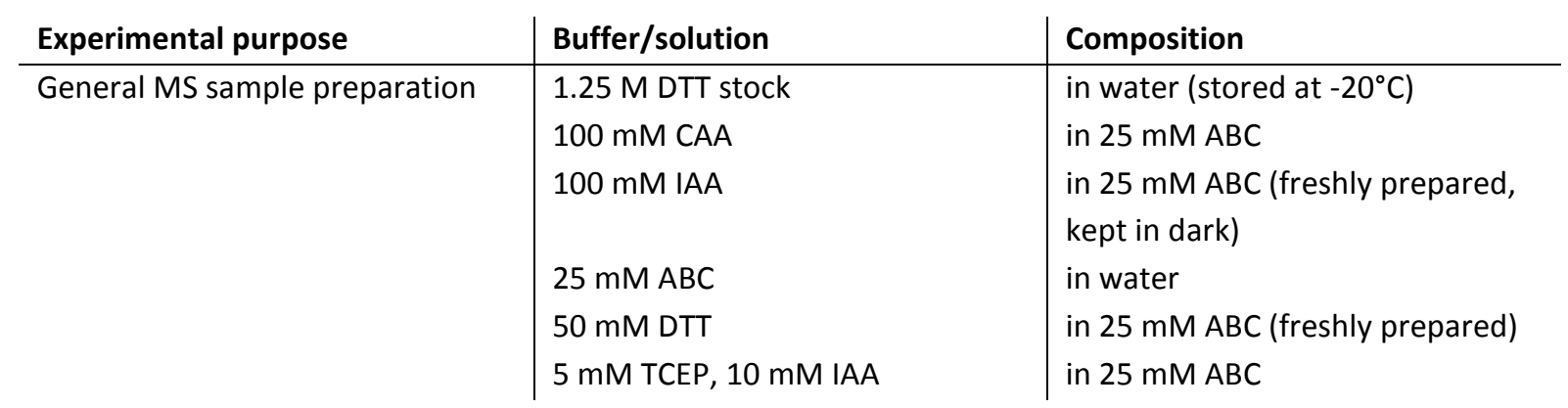


Other

PTM immunopurification (PTMScan Kits)

Phosphoproteome enrichment $\left(\mathrm{SCX}+\mathrm{TiO}_{2}\right)$

Liquid chromatography
LC-MS/MS sample loading solution

Coomassie staining solution

\section{$0.1 \mathrm{M}$ PBS $\mathrm{pH} 8$}

200 mM HEPES stock $\mathrm{pH} 8$

Bead elution buffer

StageTip purification solution $\mathrm{C}$

StageTip purification solution D

StageTip purification solution $\mathrm{E}$

Sep-Pak purification solvent $A$

Sep-Pak purification solvent $B$

Sep-Pak washing solution

SCX buffer $A$

SCX buffer B

$\mathrm{TiO}_{2}$ loading solution

$\mathrm{TiO}_{2}$ wash solution

$\mathrm{TiO}_{2}$ elution solution

$\mathrm{TiO}_{2}$ column packing solution

Buffer A (Velos, Fusion, Q

Exactive, $\mathrm{Q}$ Exactive HF)

Buffer B (Velos, Q Exactive)

Buffer B (Fusion, Q Exactive HF)
$5 \%(\mathrm{v} / \mathrm{v})$ acetonitrile, $0.1 \%(\mathrm{v} / \mathrm{v})$

formic acid and $2 \%(v / v)$

acetonitrile, $0.05(\mathrm{v} / \mathrm{v})$ TFA

$0.08 \%(w / v)$ Coomassie Brilliant

Blue G-250, 20\% (v/v) methanol,

$1.6 \%(\mathrm{v} / \mathrm{v})$ orthophosphoric acid,

$8 \%(\mathrm{w} / \mathrm{v})$ ammonium sulfate

$0.2 \mathrm{M} \mathrm{KH}_{2} \mathrm{PO}_{4}, 0.2 \mathrm{M} \mathrm{K}_{2} \mathrm{HPO}_{4}$ in water

in water, $\mathrm{pH}$ adjusted with $\mathrm{NaOH}$

$0.15 \%(v / v)$ TFA

$50 \%(v / v)$ ACN, $0.1 \%(v / v)$ TFA

$0.1 \%(v / v)$ TFA

$40 \%(\mathrm{v} / \mathrm{v})$ ACN, $0.1 \%(\mathrm{v} / \mathrm{v})$ TFA

$0.1 \%(v / v)$ TFA

$40 \%(v / v)$ ACN, 0.1\% (v/v) TFA

$5 \%(\mathrm{v} / \mathrm{v})$ ACN, $0.1 \%(\mathrm{v} / \mathrm{v})$ TFA

$10 \mathrm{mM}$ ammonium formate, $30 \%$

(v/v) ACN, pH 2.65 (adjusted with

FA)

$500 \mathrm{mM}$ ammonium formate,

$30 \%(\mathrm{v} / \mathrm{v}) \mathrm{ACN}, \mathrm{pH} 2.65$ (adjusted with FA)

$200 \mathrm{mg}$ DHB in $80 \%(\mathrm{v} / \mathrm{v}) \mathrm{ACN}, 5 \%$

(v/v) TFA

$80 \%$ (v/v) ACN, 5\% (v/v) TFA

$0.6 \mathrm{~N} \mathrm{NH}_{4} \mathrm{OH}\left(4.2 \mathrm{~mL} \mathrm{NH}_{4} \mathrm{OH}+\right.$

$95.8 \mathrm{~mL} \mathrm{H}_{2} \mathrm{O}$ )

$80 \%(v / v)$ ACN, $0.1 \%(v / v)$ TFA

$0.1 \% \mathrm{FA}$

$95 \%$ ACN, $0.1 \%$ FA

$80 \%$ ACN, $0.08 \%$ FA

\subsubsection{Consumables}

Table 5: Consumables

\begin{tabular}{l|l} 
Product & Company \\
\hline 65MMLTIFLEX-Tips 1-200 ML, round & Carl Roth, DE \\
Combitips (different sizes) & Eppendorf, DE \\
Diamond Tower Pack pipet tips $(10 \mu \mathrm{L}, 20-200 \mu \mathrm{L}, 1,000 \mu \mathrm{L})$ & Gilson, F \\
Falcon reaction tube $(15 \mathrm{~mL}, 50 \mathrm{~mL})$ & Greiner, DE \\
GELoader tip & Eppendorf, DE \\
Micro SpinColumns, C18, $5 \mu \mathrm{m}$ frit & Harvard Apparatus, USA (Serva, DE) \\
Empty Micro SpinColumns, $5 \mu \mathrm{m}$ frit & Hoefer Inc., USA (Serva, DE) \\
NuPAGE Novex 4-12\% Bis-Tris Mini Gels & Invitrogen, DE \\
Parafilm & Bemis, B \\
Safe-Lock Tubes (0.5 mL, $1.5 \mathrm{~mL}, 2 \mathrm{~mL}, 5 \mathrm{~mL})$ & Eppendorf, DE \\
Sep-Pak Vac tC18 Columns $(1 \mathrm{cc}, 3 \mathrm{cc}, 6 \mathrm{cc})$ & Waters, DE
\end{tabular}


Bottle-top and syringe sterile filters (pore size $0.2 \mu \mathrm{M}$ )

Syringe ( $1 \mathrm{~mL}, 5 \mathrm{~mL}, 10 \mathrm{~mL}, 50 \mathrm{~mL}$ )

Syringe canules (different sizes)
Sarstedt, DE

B.Braun, DE

B.Braun, DE

\subsubsection{Kits, enzymes and HPLC columns}

Table 6: Kits

\begin{tabular}{l} 
Kit \\
\hline Pierce BCA Protein Assay Kit \\
PTMScan Phospho-Tyrosine Rabbit mAb (P-Tyr-1000) \\
PTMScan Ubiquitin Remnant Motif (K- $\varepsilon-G G)$ \\
PTMScan Acetyl-Lysine Motif [Ac-K]
\end{tabular}

Table 7: Enzymes

\section{Company}

Thermo Scientific Fisher, DE

Cell Signaling Technology, DE

Cell Signaling Technology, DE

Cell Signaling Technology, DE

\begin{tabular}{l|l} 
Enzyme & Company \\
\hline AspN (sequencing grade) & Roche, DE \\
Benzonase & Novagen, USA \\
Chymotrypsin (sequencing grade) & Roche, DE \\
Rnase A & Ambion, Applied Biosystems, DE \\
Rnase T1 & Ambion, Applied Biosystems, DE \\
Trypsin (sequencing grade) & Serva Electrophoresis, DE \\
Trypsin, modified (sequencing grade) & Promega, USA
\end{tabular}

Table 8: HPLC columns

\begin{tabular}{l|l} 
Column & Company \\
\hline BioBasic SCX $2.1 \times 50 \mathrm{~mm}$ & Thermo Fisher, DE \\
Xbridge C18 $1.0 \times 150 \mathrm{~mm}, 3.5 \mu \mathrm{m}$ & Waters, IE
\end{tabular}

\subsubsection{Cell lines}

Table 9: Cell lines

\begin{tabular}{|c|c|c|c|}
\hline Cell line & Comments & Reference & $\begin{array}{l}\text { Provided by (also refer } \\
\text { to Table 12) }\end{array}$ \\
\hline $\mathrm{BL2}$ & Sporadic BL cell line & (Denépoux et al., 1997) & $\begin{array}{l}\text { University Medical } \\
\text { Center, FFM }\end{array}$ \\
\hline Daudi & $\begin{array}{l}\text { EBV-positive endemic BL; } \\
\text { Myc translocation; } \\
\text { inactivating ID3 mutation }\end{array}$ & $\begin{array}{l}\text { (Haluska et al., 1987; } \\
\text { Klein et al., 1968) }\end{array}$ & $\begin{array}{l}\text { University Medical } \\
\text { Center, FFM }\end{array}$ \\
\hline DG75 & $\begin{array}{l}\text { Sporadic BL cell line; Myc } \\
\text { translocation }\end{array}$ & $\begin{array}{l}\text { (Ben-Bassat et al., 1977; } \\
\text { Gabay et al., 1999; Penas } \\
\text { et al., 2014) }\end{array}$ & $\begin{array}{l}\text { University Medical } \\
\text { Center, FFM }\end{array}$ \\
\hline FFM_BL1 (or BL1) & $\begin{array}{l}\text { Patient derived } \mathrm{BL} \text { cells: } \\
\text { Point mutations in ELP2, } \\
\text { FOXO1, MYC and TOP } 2 A\end{array}$ & & $\begin{array}{l}\text { University Medical } \\
\text { Center, FFM }\end{array}$ \\
\hline
\end{tabular}


$\mathrm{J} 14$

KG1

MV4-11

\begin{tabular}{|l} 
Jurkat derivative SLP76 \\
deficient cell line \\
AML FLT3 WT cell line \\
AML FLT3-ITD-positive \\
cell line
\end{tabular}

(Yablonski et al., 1998)

(Koeffler and Golde, 1978)

(Santoli et al., 1987)
Technion, Haifa, Israel

University Medical

Center, FFM

University Medical

Center, FFM

\subsubsection{Software and databases}

\section{Table 10: Software}

\begin{tabular}{l|l} 
Name & Company/ Institution \\
\hline Cytoscape & Cytoscape Consortium \\
MaxQuant & Max Planck Institute for Biochemistry, DE \\
Perseus & Max Planck Institute for Biochemistry, DE \\
PhosphoPath app (for Cytoscape) & $\begin{array}{l}\text { Bijvoet Center for Biomolecular Research and Utrecht Institute } \\
\text { for Pharmaceutical Sciences, Utrecht University, NLD }\end{array}$ \\
R & R Project \\
R Studio & R studio, USA \\
Venn Diagram Plotter & Pacific Northwest National Laboratory, USA \\
Xcalibur & Thermo Fisher Scientific, DE
\end{tabular}

Table 11: Databases

\begin{tabular}{|c|c|c|c|}
\hline Name & Details & Company/Institution & Link \\
\hline BioGRID & $\begin{array}{l}\text { Protein-protein } \\
\text { interaction information }\end{array}$ & BioGRID & http://thebiogrid.org \\
\hline $\begin{array}{l}\text { DAVID Bioinformatics } \\
\text { Resources }\end{array}$ & $\begin{array}{l}\text { Gene/Protein } \\
\text { annotation, KEGG } \\
\text { pathway mapping, } \\
\text { enrichment analysis }\end{array}$ & $\begin{array}{l}\text { National Institute of } \\
\text { Allergy and Infectious } \\
\text { Diseases (NIAID), NIH }\end{array}$ & http://david.abcc.ncifcrf.gov/ \\
\hline $\begin{array}{l}\text { Human Protein } \\
\text { Reference Database } \\
\text { (HPRD) }\end{array}$ & $\begin{array}{l}\text { Protein, functional, } \\
\text { pathway and motif } \\
\text { annotation }\end{array}$ & $\begin{array}{l}\text { Johns Hopkins } \\
\text { University (Pandey Lab, } \\
\text { Institute of } \\
\text { Bioinformatics) }\end{array}$ & www.hprd.org \\
\hline Ingenuity IPA & $\begin{array}{l}\text { Protein annotation, } \\
\text { functional annotation, } \\
\text { pathway enrichment } \\
\text { analysis }\end{array}$ & Qiagen & www.ingenuity.com \\
\hline Motif-x & $\begin{array}{l}\text { Software tool for the } \\
\text { determination of } \\
\text { overrepresented amino } \\
\text { acid motifs }\end{array}$ & Harvard Medical School & $\begin{array}{l}\text { http://motif- } \\
\text { x.med.harvard.edu }\end{array}$ \\
\hline PhosphOrtholog & $\begin{array}{l}\text { Mapping of } \\
\text { orthologous sites } \\
\text { between species }\end{array}$ & $\begin{array}{l}\text { University of Sydney } \\
\text { Garvan Institute of } \\
\text { Medical Research, } \\
\text { Australia }\end{array}$ & www.phosphortholog.com \\
\hline
\end{tabular}




\begin{tabular}{|c|c|c|c|}
\hline \multirow{5}{*}{ PhosphoSitePlus (PSP) } & \multirow{5}{*}{$\begin{array}{l}\text { (Functional) annotation } \\
\text { for PTM aa sites }\end{array}$} & Victor Chang Cardiac & \multirow{5}{*}{ www.phosphosite.org } \\
\hline & & Research Institute, & \\
\hline & & Australia & \\
\hline & & Cell Signaling & \\
\hline & & Technology & \\
\hline \multirow[t]{6}{*}{ STRING } & Protein-protein & Swiss Institute of & http://string-db.org \\
\hline & interaction information & Bioinformatics, NNF & \\
\hline & & Center for Protein & \\
\hline & & Research, European & \\
\hline & & Molecular Biology & \\
\hline & & Laboratory & \\
\hline \multirow[t]{2}{*}{ TRANSFAC } & Transcription factor- & Biobase Biological & www.gene-regulation.com \\
\hline & target gene mapping & Databases, Qiagen & \\
\hline UniProtKB & $\begin{array}{l}\text { Comprehensive protein } \\
\text { database }\end{array}$ & UniProt Consortium & www.uniprot.org \\
\hline \multirow[t]{2}{*}{ WikiPathways } & Public database for & WikiPathways & www.wikipathways.org \\
\hline & biological pathways & Development team & \\
\hline
\end{tabular}

\title{
2.1.8. Collaborations
}

\author{
Table 12: Collaborations
}

\begin{tabular}{|c|c|}
\hline Project & Collaborator \\
\hline $\begin{array}{l}\text { Cell-based assays and functional experiments for the } \\
\text { analysis of phosphorylation-based signaling in } \\
\text { Burkitt's lymphoma and BTK inhibition in acute } \\
\text { myeloid leukemia; Western blotting and } \mathrm{Ca}^{2+} \text { flux } \\
\text { analysis }\end{array}$ & $\begin{array}{l}\text { Thomas Oellerich - Department of Internal Medicine, } \\
\text { Hematology/Oncology at the University Hospital } \\
\text { Frankfurt/Main, DE }\end{array}$ \\
\hline $\begin{array}{l}\text { Cell-based assays and functional experiments for } \\
\text { studying phosphorylation site analysis in TCR } \\
\text { signaling adapters }\end{array}$ & $\begin{array}{l}\text { Debbie Yablonski - 'Immune Cell Signaling Lab' at the } \\
\text { Rappaport Faculty of Medicine, Technion, Israel } \\
\text { Institute of Technology in Haifa, Israel }\end{array}$ \\
\hline $\begin{array}{l}\text { Sample preparation, MS/MS measurement, data } \\
\text { analysis for kinobead analysis in DG75 (Burkitt's } \\
\text { lymphoma) }\end{array}$ & $\begin{array}{l}\text { Bernhard Küster - Chair of Proteomics and } \\
\text { Bioanalytics at the Technical University München, } \\
\text { Freising, DE }\end{array}$ \\
\hline $\begin{array}{l}\text { RNA seq measurements for DG75 } \\
\text { Data analysis for DG75 RNA seq analysis }\end{array}$ & $\begin{array}{l}\text { Ekkehard Schütz - Chronix Biomedical, San Jose, USA } \\
\text { Tim Beißbarth - Institute of Medical Statistics, } \\
\text { University Medical Center Göttingen, DE }\end{array}$ \\
\hline
\end{tabular}

\subsection{Methods}

Cell-based sample preparation methods used to culture, label, treat and lyse cells from different experimental model systems performed by different collaborators are described in Table 12. In general, obtained protein lysates were further processed by different sample preparation techniques. Sample probes were measured by LC-MS/MS on different MS 
instruments and raw data was processed and analyzed by various data analysis tools. The mentioned steps towards (phospho)-proteomic analysis will be described in detail in the following.

\subsubsection{Cell-based sample preparation}

\subsubsection{Cell culture}

\subsubsection{Burkitt's lymphoma and acute myeloid leukemia}

All Burkitt's lymphoma (BL) and acute myeloid leukemia (AML) cell lines were cultured in RPMI 1640 (Invitrogen) supplemented with 10\% fetal calf serum (FBS; PAA), 2 mM L-glutamine (Lonza), $100 \mathrm{U} / \mathrm{ml}$ penicillin and $100 \mu \mathrm{g} / \mathrm{ml}$ streptomycin (Life Technologies) at $37^{\circ} \mathrm{C}$ and $5 \%$ $\mathrm{CO}_{2}$.

FFM_BL1 cells were isolated from a patient with BL showing bone marrow infiltration. The cells were cultured in RPMI 1640 supplemented with 20\% heat-inactivated FBS, $100 \mathrm{U} / \mathrm{ml}$ penicillin and $100 \mu \mathrm{g} / \mathrm{ml}$ streptomycin and L-glutamine (all mentioned cell culture reagents for FFM_BL1 cells were purchased from Invitrogen).

\subsubsection{TCR signaling adapters}

Jurkat derivative J14 cells (SLP76-deficient) were stably infected with Migr-strep-SLP-76 WT (Twin-Strep-Tag) and were cultured in RPMI lacking lysine, arginine and tyrosine with Lglutamine (Bet HaEmek bioindustry) supplemented with PSG, 10\% dialyzed FBS (Bet HaEmek).

\subsubsection{SILAC labeling}

\subsection{Burkitt's lymphoma and acute myeloid leukemia}

SILAC labeling of DG75, Daudi, MV4-11 and KG1 was achieved by culturing the cells in SILACRPMI 1640 medium lacking arginine and lysine (Pierce) supplemented with $10 \%$ heatinactivated dialyzed FBS (PAA), $100 \mathrm{U} / \mathrm{ml}$ penicillin and $100 \mu \mathrm{g} / \mathrm{ml}$ streptomycin as well as the respective SILAC amino acids (all Cambridge Isotopes): 'Light' (L): ${ }^{12} \mathrm{C}_{6}{ }^{14} \mathrm{~N}_{4} \mathrm{~L}$-arginine (R+0 or Arg+0), ${ }^{12} \mathrm{C}_{6}{ }^{14} \mathrm{~N}_{2}$ L-lysine (K+0 or Lys+0), 'Medium' (M): ${ }^{13} \mathrm{C}_{6}{ }^{14} \mathrm{~N}_{4}$ L-arginine (+6 Da; R+6 or Arg+6), L-lysine-4,4,5,5- $\mathrm{D}_{4}$ (+4 Da; K+4 or Lys+4) and 'Heavy' (H): ${ }^{13} \mathrm{C}_{6}{ }^{15} \mathrm{~N}_{4} \mathrm{~L}$-arginine (+10 Da; $\mathrm{R}+10$ or $\mathrm{Arg}+10),{ }^{13} \mathrm{C}_{6}{ }^{15} \mathrm{~N}_{2}$ L-lysine (+8 Da; $\mathrm{K}+8$ or Lys+8). L-arginine and L-lysine were supplemented to a final concentration of $0.132 \mathrm{mM}$ and $0.316 \mathrm{mM}$ or the doubled or tripled concentration depending on the cultivated cell type. To avoid arginine-to-proline conversion, affected cells were cultured as above with additional $200 \mathrm{mM}$ unlabeled L-proline. 


\subsubsection{TCR signaling adaptors}

The SILAC-RPMI medium lacking lysine, arginine and tyrosine was supplemented with the respective 'light' or 'heavy' amino acids: 'Light' (L): ${ }^{12} \mathrm{C}_{9}{ }^{14} \mathrm{~N}$ L-tyrosine $\left(\mathrm{Y}+0\right.$ or Tyr+O), ${ }^{12} \mathrm{C}_{6}{ }^{14} \mathrm{~N}_{4}$ L-arginine ( $\mathrm{R}+\mathrm{O}$ or Arg+0), ${ }^{12} \mathrm{C}_{6}{ }^{14} \mathrm{~N}_{2}$ L-lysine ( $\mathrm{K}+\mathrm{O}$ or Lys+0) and 'Heavy' (H): ${ }^{13} \mathrm{C}_{9}{ }^{15} \mathrm{~N}$ L-tyrosine (+10 Da; $\mathrm{Y}+10$ or Tyr+10), ${ }^{13} \mathrm{C}_{6}{ }^{15} \mathrm{~N}_{4}$ L-arginine (+10 Da; R+10 or Arg+10), ${ }^{13} \mathrm{C}_{6}{ }^{15} \mathrm{~N}_{2}$ L-lysine (+8 $\mathrm{Da} ; \mathrm{K}+8$ or Lys+8). The following concentrations were used, L-tyrosine $0.11 \mathrm{mM}$, L-lysine 0.22 $\mathrm{mM}$ and L-arginine $0.115 \mathrm{mM}$ (light amino acids: Bet Haemek, heavy amino acids: Cambridge Isotopes). Cells were adapted to the above medium for about 2 weeks, using light amino acids, and then grown in heavy or light medium for at least seven doublings and expanded to 120 to 160 million light- and heavy-labeled cells, respectively.

\subsubsection{Functional analyses}

\subsection{BCR stimulation}

For BCR stimulation DG75, Daudi and FFM_BL1 cells were starved in RPMI without supplements for 15 minutes. Subsequently, BCRs were stimulated with $10 \mu \mathrm{g} / \mathrm{ml} \mathrm{F}(\mathrm{ab}) 2$ antihuman IgM antibodies (Dianova) at $37^{\circ} \mathrm{C}$ for the durations indicated below. Light-labeled batches of DG75 cells were left in the unstimulated ground state $(0 \mathrm{~min})$ while the BCRs of the medium-labeled batches were stimulated for $2 \mathrm{~min}$ and the heavy-labeled batches were stimulated for $5 \mathrm{~min}$. For the second SILAC triplet, the medium and heavy batches were stimulated for 10 and $20 \mathrm{~min}$, respectively. Light-labeled Daudi batches were left nonstimulated ( $0 \mathrm{~min}$ ), while the BCRs of medium- and heavy-labeled cells were stimulated for 2 or $10 \mathrm{~min}$, respectively. The non-SILAC labeled FFM_BL1 cells were stimulated for the same BCR stimulation durations as Daudi cells.

For kinobead affinity purification the BCRs of non-labeled DG75 cells were left non-stimulated and were stimulated for 2, 5, 10 or 20 min, respectively. For the RNAseq analysis, the BCRs of non-labeled DG75 cells were stimulated for $10 \mathrm{~min}, 20 \mathrm{~min}, 1 \mathrm{~h}$ or $2 \mathrm{~h}$, respectively. As a control, one batch of DG75 cells was not stimulated ( $0 \mathrm{~min})$.

\subsection{BCR knockdown and Syk inhibition}

The tetracycline-inducible CD79A knockdown in DG75 cell lines was performed in light-labeled cells. Heavy-labeled DG75 cells were treated with the Syk inhibitor PRT062607 (also P505-15 or BIIB057) for one hour. 


\subsection{BRAF inhibition in $B L$}

Heavy SILAC labeled DG75 cells were pre-treated with $2 \mu \mathrm{M}$ of the BRAF inhibitor Raf265 (Selleckchem) for $45 \mathrm{~min}$ in SILAC RPMI without any supplements before addition of $10 \mu \mathrm{g} / \mathrm{ml}$ $\mathrm{F}(\mathrm{ab})_{2}$ anti-human IgM antibody (Dianova) to stimulate BCR signaling.

\subsubsection{3. $B R A F$ interactome analysis in $B L$}

$1 \times 10^{8}$ SILAC labeled (light, medium and heavy) DG75 and BL2 cells were washed once with PBS, resuspended in SILAC RPMI without any supplements and incubated at $37^{\circ} \mathrm{C}$ for $20 \mathrm{~min}$. Heavy SILAC labeled cultures were BCR stimulated for 5 min by addition of $10 \mu \mathrm{g} / \mathrm{ml} \mathrm{F}(\mathrm{ab})_{2}$ anti-human IgM antibody (Dianova) while light and medium SILAC labeled cultures were left unstimulated. Cells were harvested by centrifugation (1200 rpm, $5 \mathrm{~min}, 4^{\circ} \mathrm{C}$ ) and washed once with ice-cold PBS. Cells were lysed in $1 \mathrm{ml} \mathrm{NP-40} \mathrm{lysis} \mathrm{buffer} \mathrm{for} 10 \mathrm{~min}$ on ice. Cellular debris were removed by centrifugation ( $\left.15000 \mathrm{rpm}, 10 \mathrm{~min}, 4^{\circ} \mathrm{C}\right)$. An anti-BRAF antibody $(5 \mu \mathrm{g}$ of rabbit anti-BRAF, Bethyl or $6 \mu \mathrm{g}$ of mouse anti-Raf-B (F-7), Santa Cruz Biotechnology) was added to the lysates of medium and heavy SILAC labeled cells while the lysates of light SILAC labeled cells were left untreated. Lysates were incubated overnight at $4^{\circ} \mathrm{C}$ on a turning wheel. $50 \mu \mathrm{l}$ of protein A/G agarose beads (Santa Cruz Biotechnology) were added to all lysates and incubated for 1.5 hours at $4^{\circ} \mathrm{C}$ on a turning wheel. Beads were washed three times with lysis buffer and were boiled with NUPAGE LDS Sample at $95^{\circ} \mathrm{C}$ for $5 \mathrm{~min}$.

\subsection{Kinobead sample preparation}

DG75 cell pellets were lysed in lysis buffer containing $50 \mathrm{mM}$ Tris/ $\mathrm{HCl} \mathrm{pH} \mathrm{7.5,5 \%} \mathrm{glycerol,} 1.5$ $\mathrm{mM} \mathrm{MgCl}_{2}, 150 \mathrm{mM} \mathrm{NaCl}, 1 \mathrm{mM} \mathrm{Na}_{3} \mathrm{VO}_{4}, 0.8 \% \mathrm{NP}-40,0.375 \mathrm{mM} \mathrm{NaF}, 1 \mathrm{mM}$ DTT including protease inhibitors (Sigma-Aldrich). Cells were lysed by subsequent cycles of thawing and freezing. The lysate was ultracentrifuged for $1 \mathrm{~h}$ at $4^{\circ} \mathrm{C}$ and $145.000 \mathrm{xg}$. The supernatant was saved while the majority of the lipid layer was discarded. Kinobead pulldowns were performed as described previously by Bantscheff et al., 2007. Cell lysates were diluted with equal volumes of $1 x$ compound pulldown (CP) buffer (50 mM Tris/ $\mathrm{HCl} \mathrm{pH} \mathrm{7.5,} \mathrm{5 \%} \mathrm{glycerol,} 1.5 \mathrm{mM} \mathrm{MgCl}$, 150 $\mathrm{mM} \mathrm{NaCl}, 20 \mathrm{mM} \mathrm{NaF}, 1 \mathrm{mM}$ sodium ortho-vanadate, $1 \mathrm{mM} \mathrm{DTT}, 5 \mathrm{mM}$ calyculin $\mathrm{A}$ and protease inhibitors). Lysates were further diluted to a final protein concentration of $5 \mathrm{mg} / \mathrm{ml}$ using 1x CP buffer supplemented with $0.4 \%$ NP-40. Kinobeads (100 $\mu$ l suspension) were incubated with lysates (total of $5 \mathrm{mg}$ protein per state) at $4^{\circ} \mathrm{C}$ for $4 \mathrm{~h}$. Subsequently, beads were washed with 1x CP buffer and collected by centrifugation. Bound proteins were eluted 
with 2x NuPAGE ${ }^{\circledR}$ LDS Sample Buffer (Invitrogen) and reduced and alkylated by $10 \mathrm{mM}$ DTT and $55 \mathrm{mM}$ iodoacetamide, respectively. All samples were run $1 \mathrm{~cm}$ into a 4-12\% NuPAGE gel (Invitrogen) in order to concentrate the sample prior to in-gel trypsin digestion.

\subsection{BTK interactome and inhibition in AML}

Details about BTK immunoprecipitation in KG1 and FLT3 in MV4-11 cells and interactome analysis are described in Oellerich et al., 2013; 2015. Heavy-labeled KG1 and MV4-11 cells were treated with $500 \mathrm{nM}$ Ibrutinib and their light-labeled counterparts with DMSO as a control for one hour. Further details are described in Oellerich et al., 2015.

\subsection{TCR stimulation and SLP76 affinity purification}

Light-labeled cells were incubated in PBS (unstimulated) and heavy-labeled cells were incubated with an anti-TCR antibody C305 at a final concentration of 1:500 in PBS for the respective time durations ( $1 \mathrm{~min}, 2 \mathrm{~min}, 5 \mathrm{~min}$ and $10 \mathrm{~min}$ ). Cells were lysed for $15 \mathrm{~min}$ on ice with $1.3 \mathrm{~mL}$ lysis buffer containing $20 \mathrm{mM}$ HEPES pH 7.3, 1\% Triton, $150 \mathrm{mM} \mathrm{NaCl}, 1 \mathrm{mM}$ EGTA, $10 \mathrm{mM} \mathrm{NaF}, 1 \mathrm{mM} \mathrm{Na}_{3} \mathrm{VO}_{4}, 50 \mathrm{mM} \beta$-glycerol phosphate, $20 \mathrm{mM}$ sodium pyrophosphate, 10 $\mu \mathrm{g} / \mathrm{ml}$ aprotinin, $10 \mu \mathrm{g} / \mathrm{ml}$ leupeptin, $2 \mathrm{mM}$ PMSF, 10\% glycerol and $1 \mathrm{mM}$ DTT. Lysates were cleared by $10 \mathrm{~min}$ centrifugation at max. speed at $4^{\circ} \mathrm{C}$ and the supernatant was removed. 600 $\mu l$ of the respective lysates, corresponding to 120 million cells were mixed with $120 \mu \mathrm{l}$ prewashed streptactin beads and were incubated for $1 \mathrm{~h}$ at $4^{\circ} \mathrm{C}$. Bound proteins were eluted with 1.5x NuPAGE sample buffer containing $10 \%$ (v/v) NuPAGE reducing agent by heating for $6 \mathrm{~min}$ at $85^{\circ} \mathrm{C}$. The yield was validated by SDS-PAGE and confirmed by immunoblotting with antibodies directed against SLP76 (LCP2) and GADS (GRAP2).

\subsubsection{Cell lysis}

Depending on the sample preparation method, different cell lysis approaches, if not already done during functional analysis, were required. For proteomics and global phosphoproteomics analysis, SILAC labeled cells $\left(5 \times 10^{7}-10^{8}\right)$ were lysed in a NP-40-based lysis buffer (50 mM Tris- $\mathrm{HCl}$ pH 7.5-7.8, $150 \mathrm{mM} \mathrm{NaCl}$, 0.5\% NP-40, $5 \mathrm{mM} \mathrm{NaF,} 1$ mM Na $\mathrm{VO}_{4}$, Complete Protease Inhibitor Cocktail (Roche)) for $10 \mathrm{~min}$ on ice. Cellular debris was removed by centrifugation ( $15000 \mathrm{rpm}, 10 \mathrm{~min}, 4^{\circ} \mathrm{C}$ ). Protein concentrations were determined with the BCA Protein Assay Kit (Pierce) according to the manufacturer's protocol and stored at $-80^{\circ} \mathrm{C}$ until use. 
Cell lysis for antibody-based PTM enrichment was performed according to the manufacturer's instruction (PTMScan Kit, Cell Signaling Technology) using an urea-based lysis buffer. Briefly, $1 \times 10^{8}$ cells were washed once with PBS and lysed in $10 \mathrm{ml}$ of urea lysis buffer (20 mM HEPES $\mathrm{pH}$ 8.0, $9 \mathrm{M}$ urea, $1 \mathrm{mM}$ sodium orthovanadate, $2.5 \mathrm{mM}$ sodium pyrophosphate, $1 \mathrm{mM} \beta$ glycerophosphate). Samples were stored at $-80^{\circ} \mathrm{C}$ until further processing.

\subsubsection{Sample preparation for LC-MS/MS analysis}

\subsubsection{Proteomic analysis}

For protein expression analysis, the protein concentration was estimated with a BCA assay for SILAC labeled or label-free cell lysates (in NP-40 lysis buffer). For SILAC labeled cell lines, equimolar amounts of each labeling state were mixed. For label-free cells each experimental condition was treated independently. A total of $150 \mu \mathrm{g}$ protein was mixed with SDS sample buffer and heated for $10 \mathrm{~min}$ at $70^{\circ} \mathrm{C}$. Proteins were separated with SDS-PAGE for $50 \mathrm{~min}$ at 200 V using precast Bis-Tris minigels and visualized by staining with Coomassie Brilliant Blue overnight. In-gel digestion was performed as described previously by Shevchenko et al. with minor modifications (Shevchenko et al., 2007). Briefly, after gel destaining, each lane was cut into 23 slices with an in-house made gel cutter, reduced with $10 \mathrm{mM} \mathrm{DTT} \mathrm{for} 50 \mathrm{~min}$ at $56^{\circ} \mathrm{C}$ and alkylated with $55 \mathrm{mM}$ IAA for $20 \mathrm{~min}$ at $37^{\circ} \mathrm{C}$ in the dark. Proteins were digested overnight in-gel with trypsin, peptides were extracted, dried in a SpeedVac apparatus and subjected to mass spectrometric analysis.

\subsubsection{PTM analysis}

For the enrichment of PTMs several workflows were used. Global phosphoproteomic analyses of Burkitt's lymphoma and the acute myeloid leukemia cells were done by strong cation exchange (SCX) followed by titanium dioxide $\left(\mathrm{TiO}_{2}\right)$ enrichment. To specifically enrich peptides harboring phosphorylated tyrosine residues in the Burkitt's lymphoma and the acute myeloid leukemia project, specific antibodies directed against phosphotyrosine were used. Additionally, acetylation and ubiquitination enrichment for the Burkitt's lymphoma project was performed with PTM-specific antibodies. For the investigation of phosphorylation sites on purified TCR signaling proteins, SDS-PAGE together with $\mathrm{TiO}_{2}$ enrichment was performed. 


\subsection{Global phosphoproteomic analysis}

Protein concentration of each SILAC state was determined by BCA assay according to manufacturer's recommendations. SILAC labeled protein lysates were mixed in equimolar amounts, diluted 1:4 with $25 \mathrm{mM} \mathrm{ABC}$ buffer and treated with $1 \mathrm{U}$ Benzonase for hydrolysis of all nucleic acids for $1 \mathrm{~h}$ at $37^{\circ} \mathrm{C}$. Proteins were precipitated by adding fivefold volume of ice cold acetone and sequent storage of samples for at least one hour at $-20^{\circ} \mathrm{C}$. The precipitate was pelleted by centrifugation for $30 \mathrm{~min}$ with $\max . \mathrm{rpm}$ at $4^{\circ} \mathrm{C}$. The acetone was removed and the pellet was washed with $80 \%$ ice cold $\mathrm{EtOH}(\mathrm{v} / \mathrm{v})$ for $10 \mathrm{~min}$ with $\max$. rpm at $4^{\circ} \mathrm{C}$. The $80 \% \mathrm{EtOH}$ was removed and the pellet was subsequently dried. After drying, the pellet was dissolved by sonication in 1\% RapiGest Surfactant (w/v) in $25 \mathrm{mM}$ ammonium bicarbonate for at least one to six $\mathrm{h}$. Dissolved proteins were reduced at a final concentration of $10 \mathrm{mM}$ DTT for $1 \mathrm{~h}$ at $65^{\circ} \mathrm{C}$ and reduced with CAA at a final concentration of $20 \mathrm{mM}$ for $1 \mathrm{~h}$ at $37^{\circ} \mathrm{C}$. Proteins were digested with trypsin at a 1:20 to 1:100 trypsin-to-protein (w/w) ratio in the presence of $0.1 \%$ RapiGest at $37^{\circ} \mathrm{C}$ overnight. For RapiGest degradation, the digest was acidified to $2 \% \mathrm{FA}$ or $1 \%$ TFA and incubated for $30 \mathrm{~min}$ to $2 \mathrm{~h}$ at $37^{\circ} \mathrm{C}$. RapiGest precipitations were cleared by centrifugation with max. rpm at RT. The peptide containing supernatant was taken to dryness in a SpeedVac concentrator. Peptides were fractionated by strong cation exchange (SCX) as described by Gruhler et al. with some modifications (Gruhler et al., 2005).

Briefly, SCX chromatography was performed on a FPLC system (SMART, Pharmacia) using an ammonium formate based buffer system. Buffer $A$ had a final concentration of $10 \mathrm{mM}$ ammonium formate and buffer $B$ had a final concentration of $500 \mathrm{mM}$ ammonium formate both at pH 2.7 and containing 30\% ACN (v/v). 20 Fractions were collected over a 50 min gradient with a flow rate of $100 \mu \mathrm{L} / \mathrm{min}$. The first twelve fractions were dried in a SpeedVac concentrator and subsequently used for phosphopeptide enrichment or stored at $-20^{\circ} \mathrm{C}$ until use. The following $\mathrm{TiO}_{2}$ spin column phosphopeptide enrichment was performed with minor modifications as described by Larsen et al. (Larsen et al., 2005b). Dried peptides were suspended by intensive vortexing and sonication in $60 \mu \mathrm{L}$ of loading solution. All following centrifugation steps were performed with $3000 \mathrm{rpm}$ at RT for $5 \mathrm{~min}$. In-house made titanium dioxide spin columns were equilibrated with $60 \mu \mathrm{L}$ of loading solution followed by sample loading. Phosphopeptides bound to $\mathrm{TiO}_{2}$ beads were washed three times with $60 \mu \mathrm{L}$ loading solution and five times with $60 \mu \mathrm{L}$ washing solution. Phosphopeptides were eluted with a $0.6 \mathrm{~N}$ 
solution of $\mathrm{NH}_{4} \mathrm{OH}(\mathrm{pH} \geq 10.5)$, dried in a SpeedVac concentrator and stored at $-20^{\circ} \mathrm{C}$ until mass spectrometric analysis.

\subsubsection{Antibody-based PTM enrichment}

Antibody-based enrichment for tyrosine-phosphorylated peptides was performed using the PTMScan Phospho-Tyrosine rabbit mAB (P-Tyr-1000) Kit as described in the manufacturer's instructions and according to Rush et al. (Cell Signaling Technology) (Rush et al., 2004a).

Briefly, DNA was removed by microtip sonication on ice in three $15 \mathrm{~s}$ intervals with an amplitude of $50 \%$ and an one min break in between. Debris and precipitations were removed by centrifugation at $14 \mathrm{kxg}$ at $15^{\circ} \mathrm{C}$ for $30 \mathrm{~min}$. Aliquots for SDS-PAGE-based digestion controls were taken and proteins were reduced with DTT at a final concentration of $4.5 \mathrm{mM}$ at $55^{\circ} \mathrm{C}$ for $30 \mathrm{~min}$. Reduced cysteine residues were alkylated with IAA at a final concentration of 10 $\mathrm{mM}$ at RT for $10 \mathrm{~min}$ in the dark. The protein lysate was diluted 1:4 with $20 \mathrm{mM}$ HEPES pH 8 to reduce the urea concentration to $2 \mathrm{M}$. Trypsin was added in a 1:20 to 1:100 trypsin-toprotein mass ratios and incubated overnight at $37^{\circ} \mathrm{C}$. The digest was acidified to $1 \%$ TFA final concentration; precipitations were removed by centrifugation with 1,780 xg at RT for $15 \mathrm{~min}$. Peptides were purified with Sep-Pak tC18 columns (1cc or $6 c c$, Waters). The $1 \mathrm{cc}$ columns were used for centrifugation based desalting, whereas the $6 \mathrm{cc}$ columns were used for gravity flow purification. Sep-Pak columns were equilibrated in a first step with $100 \%$ ACN, followed by $0.1 \%$ TFA. The acidified sample was loaded on the columns; bound peptides were washed with $0.1 \%$ TFA and 5\% ACN, 0.1\% TFA. Purified peptides were eluted with $40 \%$ ACN, $0.1 \%$ TFA, subsequently frozen in a bulb, stored at $-20^{\circ} \mathrm{C}$ at least for $2 \mathrm{~h}$, lyophilized until dryness and stored at $-20^{\circ} \mathrm{C}$ until use. Lyophilized peptides were re-suspended in $1.4 \mathrm{~mL} 1 \mathrm{x}$ IAP buffer (supplied by Cell Signaling Technology), the $\mathrm{pH}$ was controlled and precipitates were removed by centrifugation at $10 \mathrm{k} \mathrm{xg}$ at $4^{\circ} \mathrm{C}$ for $5 \mathrm{~min}$. All following centrifugation-based washing steps were performed with a maximum of $2 \mathrm{k} \mathrm{xg}$ at $4^{\circ} \mathrm{C}$ for $30 \mathrm{~s}$. The antibody-bead slurry was washed four times with $1 \mathrm{~mL}$ PBS followed by incubation with the purified peptides for at least $2 \mathrm{~h}$ at $4^{\circ} \mathrm{C}$ on an head-over-tail rotator. After incubation, the supernatant was removed; beads were washed two times with 1x IAP buffer and three times with LiChroSolv water. Antibodypeptides were eluted by incubation of the antibody-bead-peptide slurry with $0.15 \%$ TFA for $10 \mathrm{~min}$. The eluate was removed and a second elution step was performed. In order to remove the antibody from phosphorylated peptides, StageTip purification was applied as follows: 5 layers of C18 Empore Octadecyl material were placed in a GELoader tip. C18 material was 
equilibrated with solution C, followed by sample loading. The bound sample was washed two times with solution $D(0.1 \%$ TFA) and purified phosphotyrosine enriched peptides were eluted with solution $\mathrm{E}(40 \% \mathrm{ACN}, 0.1 \% \mathrm{TFA})$. The eluate was dried in a SpeedVac apparatus and stored at $-20^{\circ} \mathrm{C}$ until mass spectrometric measurement. Ubiquitinated and/or acetylated peptides were enriched using the manufacturer's instructions and the procedure described above with the difference that the PTMScan Acetyl-Lysine Motif $[\mathrm{Ac}-\mathrm{K}]$ and the Ubiquitin Remnant Motif (K-ع-GG) antibody kits were used.

Experiments were conducted by Kai-Hsin Chan and Zivojin Jevtic (MSc Molecular Biology Program of the International Max Planck International Research School) under the supervision of Jasmin Corso within a lab rotation internship from the $5^{\text {th }}$ of January 2015 until the $27^{\text {th }}$ of Febuary 2015.

\subsubsection{Phosphoproteomic analysis of TCR signaling adapters}

For the analysis of phosphorylation sites of the TCR signaling adapters SLP76 and GADS, equimolar mixed light- and heavy-labeled cell lysates after SLP76 affinity purification were separated with SDS-PAGE for 50 min at $200 \mathrm{~V}$ using precast Bis-Tris minigels (NuPAGE Novex 4-12\%, Life Technologies). For each TCR stimulation time point (1 min, $2 \mathrm{~min}, 5$ and $10 \mathrm{~min}$ ), three lanes were run on the gel. Proteins were visualized by staining with Coomassie Brilliant Blue (Serva) overnight. After gel destaining, gel bands corresponding to GRAP2 (GADS) at 37.9 kDa and LCP2 (SLP76) at 60.1 kDa were excised over a wide range and cut into small quadratic pieces, respectively. Proteins were reduced with $10 \mathrm{mM} \mathrm{DTT}$ for $50 \mathrm{~min}$ at $56^{\circ} \mathrm{C}$, alkylated with $55 \mathrm{mM}$ IAA (both Sigma-Aldrich) for $20 \mathrm{~min}$ at $37^{\circ} \mathrm{C}$ in the dark. Samples were digested with $0.75 \mu \mathrm{g}$ of the endoproteinases trypsin (Promega), AspN or chymotrypsin (both Roche) per gel slice overnight at $25^{\circ} \mathrm{C}$ (Chymotrypsin) or $37^{\circ} \mathrm{C}$ (Trypsin and AspN). After extraction from the gel, peptides were dried in a SpeedVac apparatus and stored until enrichment by $\mathrm{TiO}_{2}$ microcolumns. Dried peptides were suspended in $60 \mu \mathrm{L}$ of $200 \mathrm{mg} / \mathrm{mL}$ dihydrobenzoic acid (DHB, Sigma-Aldrich) in 80\% ACN, 5\% TFA. All following centrifugation steps were performed with $3000 \mathrm{rpm}$ at RT for $5 \mathrm{~min}$. In-house made titanium dioxide (GL Science, Japan) spin columns were equilibrated one time with $80 \%$ ACN, $5 \%$ TFA followed by sample loading. The $\mathrm{TiO}_{2}$ bound peptides were washed three times with solution $B$ containing $200 \mathrm{mg} / \mathrm{mL} \mathrm{DHB}$ in $80 \%$ ACN, 5\% TFA followed by five washes with $80 \%$ ACN, 5\% TFA. Phosphopeptides were eluted with a $0.6 \mathrm{~N}$ solution of $\mathrm{NH}_{4} \mathrm{OH}(\mathrm{pH} \geq 10.5)$, dried in a SpeedVac concentrator and stored at $-20^{\circ} \mathrm{C}$ until mass spectrometric measurement. 


\subsubsection{LC-MS/MS analysis}

In the following paragraphs, MS instruments that were used to perform LC-MS/MS analysis of the before mentioned samples are described. All MS instruments used for this work were obtained from the instrument manufacturer Thermo Scientific. The LTQ Orbitrap Velos, the Q Exactive, the Q Exactive HF and the Orbitrap Fusion were operated by the Bioanalytical Mass Spectrometry Group at the Max Planck Institute for Biophysical Chemistry (Table 2). The Orbitrap Elite was operated within the collaboration with Bernhard Küster as mentioned previously (Table 12). In each paragraph, the generalized, most important instrument operating parameters are described. Detailed LC-MS/MS instrumental settings for each sample can be found in the Appendix.

\subsubsection{LTQ Orbitrap Velos}

Peptide fractions were analyzed on a nanoflow liquid chromatography system (Agilent 1100 series, Agilent) coupled to a hybrid linear ion trap-Orbitrap mass spectrometer (LTQ Orbitrap Velos, Thermo Scientific). Samples were pre-concentrated and desalted on a trap column (100 $\mu \mathrm{m} \times 20 \mathrm{~mm}$, ReproSil-Pur 120 C18-AQ, $5 \mu \mathrm{m}$, Dr. Maisch GmbH, packed in-house) at $5 \mu \mathrm{l} / \mathrm{min}$ in loading buffer ( $2 \%$ ACN, 0.1\% FA). Peptides were separated on an analytical column (75 $\mu \mathrm{m}$ x 200 mm, ReproSil-Pur 120 C18-AQ, 3 mm, Dr. Maisch GmbH, packed in-house) using different linear gradients (see Appendix) from $5 \%$ to $34 \%$ buffer B (95\% ACN, 0.1\% FA) at a flow rate of $300 \mathrm{nl} / \mathrm{min}$. The mass spectrometer was operated in data-dependent acquisition (DDA) mode. Full scan spectra from $\mathrm{m} / \mathrm{z} 350-1600$ were acquired in the Orbitrap with a resolution of 30,000 FWHM at $m / z 400$. Product ion spectra of the ten most abundant precursors per cycle were acquired. Collision induced dissociation (CID) of selected precursors with charge states $2+$ to $4+$ was performed in the linear ion trap with normalized collision energy (NCE) of 35\% and an isolation window of $2 \mathrm{~m} / \mathrm{z}$. The dynamic exclusion time was set to $60 \mathrm{~s}$, the automatic gain control (AGC) target value and maximum injection time (max. IT) for MS and MS/MS were $1 \times 10^{6}$ in $500 \mathrm{~ms}$ and between $5 \times 10^{4}$ and $1 \times 10^{5}$ in $100 \mathrm{~ms}$, respectively.

\subsubsection{Q Exactive}

Samples were measured on an EASY n-LC 1000 (Thermo Scientific) coupled to a hybrid quadrupole-Orbitrap mass spectrometer ( $Q$ Exactive, Thermo Scientific). The samples were pre-concentrated and desalted on a trap column (100 $\mu \mathrm{m} \times 20 \mathrm{~mm}$, ReproSil-Pur 120 C18-AQ, $5 \mu \mathrm{m}, \mathrm{Dr}$. Maisch $\mathrm{GmbH}$, packed in-house) at $5 \mu \mathrm{l} / \mathrm{min}$ in loading buffer ( $2 \% \mathrm{ACN}, 0.1 \% \mathrm{FA}$ ). 
Peptides were separated on an analytical column (75 $\mu \mathrm{m} \times 200 \mathrm{~mm}$, ReproSil-Pur 120 C18-AQ, $3 \mu \mathrm{m}$, Dr. Maisch $\mathrm{GmbH}$, packed in-house) using linear gradients (see Appendix) with an increasing \%age of buffer $B(95 \%$ ACN, $0.1 \% F A)$ and a decreasing \%age buffer $A(0.1 \% \mathrm{FA})$ at a flow rate of $300 \mathrm{nl} / \mathrm{min}$. The Q Exactive was operated in DDA selecting the top most abundant precursors (see Appendix) for higher-energy collisional dissociation (HCD) in the collision cell with an isolation window of $2 \mathrm{~m} / \mathrm{z}$ and a NCE of $28 \%$. Full scan spectra from $\mathrm{m} / \mathrm{z} 350-1600$ were acquired with a MS resolution setting of 70,000 at $\mathrm{m} / \mathrm{z} 200$ and fragment spectra with a MS/MS resolution of 17,000 or 35,000 in the Orbitrap (see Appendix). The AGC target value and max. IT for MS and MS/MS were set to $1 \times 10^{6}$ in $60 \mathrm{~ms}$ and $1 \times 10^{4}$ or $2 \times 10^{5}$ in 50,60 or 120 ms, respectively.

\subsubsection{Q Exactive HF}

Peptides were dissolved in 2\% ACN, $0.05 \%$ TFA and were analyzed with an Ultimate 3000 RSLC UHPLC (Dionex, USA) coupled to a Q Exactive HF (Thermo Fisher). Peptides were concentrated on a precolumn (100 $\mu \mathrm{m} \times 30 \mathrm{~mm}$, ReproSil-Pur $120 \mathrm{C} 18-\mathrm{AQ}, 3 \mu \mathrm{m}$, Dr. Maisch GmbH, packed in-house) and separated on an analytical column (75 $\mu \mathrm{m} \times 280 \mathrm{~mm}$, ReproSil-Pur 120 C18-AQ, $1.9 \mu \mathrm{m}$, Dr. Maisch GmbH, packed in-house) with a linear gradient of increasing buffer B (80\% $\mathrm{ACN}, 0.08 \% \mathrm{FA})$ and decreasing buffer $\mathrm{A}(0.1 \% \mathrm{FA}$ in water) at constant flow rate of $300 \mathrm{nl} / \mathrm{min}$. The Q Exactive HF mass spectrometer was operated in DDA top 15, 20 or top 30 methods with a MS survey scan resolution of 120,000 and a MS/MS resolution of 15,000 or 30,000. HCD was performed with NCE of $28 \%$ and isolation window of 1.4 to $2 \mathrm{~m} / \mathrm{z}$. Charge states rejected for fragmentation and the dynamic exclusion time settings can be found in the Appendix. AGC target value and max. IT for MS and MS/MS were $1 \times 10^{6}$ in $50 \mathrm{~ms}$ or $80 \mathrm{~ms}$ and $1 \times 10^{5}$ in 50 to $80 \mathrm{~ms}$, respectively.

\subsubsection{Orbitrap Fusion}

Peptides were analyzed with an Ultimate 3000 RSLC UHPLC (Dionex, USA) coupled to an Orbitrap Fusion mass spectrometer (Thermo Fisher Scientific). Peptides were concentrated on a precolumn (100 $\mu \mathrm{m} \times 30$ mm, ReproSil-Pur 120 C18-AQ, $3 \mu \mathrm{m}$, Dr. Maisch GmbH, packed inhouse) and separated on an analytical column (75 $\mu \mathrm{m}$ x $280 \mathrm{~mm}$, ReproSil-Pur 120 C18-AQ, $1.9 \mu \mathrm{m}$, Dr. Maisch $\mathrm{GmbH}$, packed in-house) with a $74 \mathrm{~min}$ linear gradient of increasing buffer B ( $80 \%$ ACN, $0.08 \% \mathrm{FA})$ from $2 \%$ to $44 \%$ and decreasing buffer $\mathrm{A}$ ( $0.1 \% \mathrm{FA}$ in water) at constant flow rate of $300 \mathrm{nl} / \mathrm{min}$. The Orbitrap Fusion mass spectrometer was operated with the 'top 
speed' option with a cycle time of $3 \mathrm{~s}$ and MS survey scan resolution of 120,000 and a MS/MS resolution 30,000 . HCD was performed in the multipole with NCE of $30 \%$ and isolation window of $1.4 \mathrm{~m} / \mathrm{z}$. Charge states 2 to 5 were included for fragmentation and the dynamic exclusion time was set to $20 \mathrm{~s}$. AGC target value and max. IT for MS and MS/MS were $5 \times 10^{5}$ in $50 \mathrm{~ms}$ and $1 \times 10^{4}$ in $128 \mathrm{~ms}$, respectively.

\subsubsection{Orbitrap Elite}

The kinobead analysis was performed on an Eksigent nanoLC-Ultra 1D+ (Eksigent, Dublin, CA) coupled to an Elite-Oribtrap (Thermo Scientific, Bremen, Germany). Peptides were delivered to a trap column $(100 \mu \mathrm{m} \times 20 \mathrm{~mm}$, Reprosil PUR C18-AQ, $5 \mu \mathrm{m}$, Dr. Maisch GmbH, packed inhouse) at a flow rate of $5 \mathrm{\mu L} / \mathrm{min}$ in $100 \%$ buffer $\mathrm{A}$ (0.1\% FA in HPLC grade water). After $10 \mathrm{~min}$ of loading and washing, peptides were transferred to an analytical column (75 $\mu \mathrm{m} \times 400 \mathrm{~mm}$, Reprosil PUR C18-AQ, $3 \mu \mathrm{m}$, Dr. Maisch GmbH, packed in-house) and separated using a 210 minute gradient from $2 \%$ to $35 \%$ of buffer $B(0.1 \%$ FA in ACN) at $300 \mathrm{~nL} / \mathrm{min}$ flow rate. The Orbitrap Elite was operated in DDA mode, automatically switching between MS1 and MS2 scans. Full scan MS spectra were acquired in the Orbitrap at 30,000 resolution. Internal calibration was performed using the ion signal $\left(\mathrm{Si}\left(\mathrm{CH}_{3}\right)_{2} \mathrm{O}\right) 6 \mathrm{H}^{+}$at $\mathrm{m} / \mathrm{z} 445.120025$ present in ambient laboratory air. Tandem mass spectra were generated for up to 15 peptide precursors in the linear ion trap for fragment by using HCD.

\subsubsection{MS raw file processing}

The output from one LC-MS/MS run from the above mentioned instruments is a raw file containing amongst others information about retention time, $\mathrm{m} / \mathrm{z}$ values, intensities and the charge state of intact peptide precursors and product ions of selected precursors. In order to identify and quantify peptides, proteins, and PTM sites, the raw files were submitted to the software MaxQuant. MS/MS spectra were searched against a human proteome database with the search engine Andromeda which is implemented in MaxQuant (Cox and Mann, 2008; Cox et al., 2011). In the following paragraph, raw file processing for the different projects are described.

\subsubsection{BL experiments}

All raw files were processed using MaxQuant (Versions between 1.5.0.25 and 1.5.2.8) against a UniProtKB/Swiss-Prot human database containing 88,993 and 89,706 entries (downloaded July 2014 or January 2015), respectively (Cox and Mann, 2008). Raw files of the protein 
expression, global phosphoproteome and pYome experiments and different cell lines were processed either independently from each other or categorically grouped e.g. all pYomes or all files of one cell line together. Cysteine carbamidomethylation was set as fixed modification, methionine oxidation and serine, threonine and tyrosine phosphorylation for both the pYome and the global phosphoproteome dataset as variable modification. The 'Minimum Andromeda Score' and 'Delta score' for modified peptides was set to 40 and 6, respectively. The following parameters were applied: the MS1 first search peptide tolerance was set to $20 \mathrm{ppm}$ and the main search peptide tolerance to $4.5 \mathrm{ppm}$. The FTMS MS/MS tolerance was set to $20 \mathrm{ppm}$, a false discovery rate (FDR) of $1 \%$ for peptide spectrum matches (PSM), protein and site decoy was applied, 7 amino acids as minimum peptide length and 2 maximum missed cleavages were allowed. Multiplicity was set to two in a double or to three in a triple SILAC-based quantitation experiments (duplex label: Lys+0 Arg+0, Lys+8 Arg+10; additionally for triplex labeling Lys+4 Arg+6). The 'Re-quantify' option of MaxQuant was enabled. Unique, razor, unmodified, Nterminally acetylated and methionine oxidized peptides were used for protein quantitation and the minimum ratio count required was two. For label-free quantitation, the MaxLFQ algorithm was enabled with a LFQ min. ratio count two (Cox et al., 2014). Additionally, the 'Match between runs' function was enabled at default settings of a match time window of 0.7 min and an alignment time window of $20 \mathrm{~min}$.

\subsubsection{Kinobead analysis}

Progenesis software (Version 3.1; Nonlinear Dynamics, Newcastle, UK) was used for intensitybased label-free quantitation. Briefly, after selecting one sample as a reference, the retention times of all eluting precursor $\mathrm{m} / \mathrm{z}$ values in all other samples within the experiment were aligned creating a large list of 'features' representing the same peptide in each sample. Features with two to five charges were included; features with two or less isotopes were excluded for further analysis. After alignment and feature filtering, raw abundances of all features were normalized to determine a global scaling factor for correcting experimental variation such as differences in the quantity of protein loaded into the instrument. Given that multiple MS/MS spectra are frequently collected for the same feature (precursor ion) across all the samples, the precursor intensities were ranked, and the MS/MS spectra of the five most intense precursors for each feature were transformed into peak lists and exported to generate Mascot generic files. The Mascot generic files were searched against the Uniprot human protein sequence database (Version 22.07.13, containing 88,354 entries) using Mascot 
(Version 2.2, Matrix Science, London, UK). Search parameters were set as follows: carbamidomethylation of cysteine residues as fixed modification, serine, threonine, and tyrosine phosphorylation and methionine oxidation as variable modification, trypsin as proteolytic enzyme with up to two miss cleavages, precursor ion mass tolerance of $10 \mathrm{ppm}$, fragment ion mass tolerance of $0.05 \mathrm{Da}$, decoy search enabled. Search results for spectrum to peptide matches were exported in $\mathrm{xml}$ format and then imported into Progenesis software to enable the combination of peptide quantitation and identification. Peptides with Mascot ion scores $<25$ were filtered out, and only unique peptides for corresponding proteins were used for identification and quantitation. For protein quantitation, the feature intensities of all unique peptides of a protein were summed up.

\subsubsection{AML experiments}

The protein expression and pYome raw files were analyzed with MaxQuant v1.3.0.5 and the GP was analyzed with MaxQuant v1.5.0.25 against a UniProtKB/Swiss-Prot human database containing 88,993 or 89,706 entries (downloaded July 2014 or January 2015). Raw files of KG1 and MV4-11 cells were processed together whereas protein expression, global phosphoproteome and pYome experiments were processed independently from each other. Cysteine carbamidomethylation was set as fixed modification, methionine oxidation and serine, threonine and tyrosine phosphorylation for both the pYome and the global phosphoproteome datasets as variable modification. The 'Minimum Andromeda Score' and 'Delta score' for modified peptides was set to 40 and 6, respectively for the global phosphoproteome (analyzed with MaxQuant v1.5.0.25). The following parameters were applied: the MS1 first search peptide tolerance was set to $20 \mathrm{ppm}$ and the main search peptide tolerance to $4.5 \mathrm{ppm}$ for the global phosphoproteome (Protein expression and pYome were analyzed with MaxQuant v1.3.0.5: 6 ppm), respectively. The FTMS MS/MS tolerance was set to $20 \mathrm{ppm}$, a FDR of $1 \%$ for PSM/peptide, protein and site levels was applied. Seven amino acids as minimum peptide length and maximum two missed cleavages were allowed. Multiplicity was set to two (duplex label: Lys+0 Arg+0, Lys+8 Arg+10). The 'Re-quantify' option of MaxQuant was enabled and the minimum ratio count required was set to two.

\subsubsection{TCR signaling adapters}

Raw files from four independent biological replicates were processed together as distinguishable experimental groups using MaxQuant (Version 1.5.2.8) against a 
UniProtKB/Swiss-Prot human database containing 89,706 entries (downloaded January, 2015). Raw files of different biological replicates were defined in experimental groups and different proteinase specificities were defined in parameter groups. Cysteine carbamidomethylation was set as fixed modification, methionine oxidation and serine, threonine and tyrosine phosphorylation as variable modification. The 'Minimum Andromeda Score' and 'Delta score' for modified peptides was set to 40 and 6, respectively. The following parameters were applied: the MS1 first search peptide tolerance was set to $20 \mathrm{ppm}$ and the main search peptide tolerance to $4.5 \mathrm{ppm}$, respectively. The FTMS MS/MS tolerance was set to $20 \mathrm{ppm}$, the decoy mode was set to revert, a FDR of $1 \%$ for PSM, protein and phosphorylation site was applied, seven amino acids as minimum peptide length and maximum two missed cleavages were allowed. Multiplicity was set to two: Lys+0 Arg+0 Tyr+0 and Lys+8 Arg+10 Tyr+10. Unique, razor, unmodified, N-terminally acetylated and methionine oxidized peptides were used for protein quantitation and the minimum ratio count required was two. The 'Re-quantify' option of MaxQuant was enabled.

\subsubsection{Data analysis}

In the following the data analysis pipelines for each project are described. In general, class I phosphorylation sites (true for all PTM sites) are defined as p-sites with a localization probability greater than 0.75 (Olsen et al., 2006). These p-sites are considered as localized with high confidence to a particular amino acid residue. Furthermore, a multiplicity (not to be confused with the SILAC multiplexing MaxQuant search parameters) of greater than 1 indicates the presences of an additional phosphorylation on a peptide. Therefore, in some cases same $p$-sites can be listed as mono- or multiply-phosphorylated peptide (multiplicity column in the supplementary tables). This information is of biological importance in case of combinatorial phosphorylation for example the TXY motif phosphorylation of MAP-kinases (Burack and Sturgill, 1997). Unique or non-redundant $p$-sites refer to a list of $p$-sites where same positions with different multiplicity were removed.

\subsubsection{DG75 BCR stimulation time course}

Downstream data analysis of the resulting proteinGroups.txt and Phospho(STY)Sites.txt tables from the DG75 BCR stimulation time course experiment was performed with Perseus (Versions 1.5.0.15 to 1.5.2.6). 'Reverse' and 'contaminant' (in the proteinGroups.txt also 'identified only by site' entries) were removed as well as phosphorylation sites with a global 
localization probability lower than 0.75 . P-sites were considered as quantified if at least $50 \%$ of biological replicates had a quantitation ratio reported. The binary logarithm of normalized $\mathrm{p}$-site and protein SILAC ratios and the logarithm to the base 10 of intensity values were derived. For quality control, multi-scatter plots displaying Pearson's correlation coefficients were generated for technical and biological replicates (data not shown). P-site ratios for time point $0 \mathrm{~min}$ (no stimulation) were set to 0 . For each time point, median ratios were extracted and used for further analysis. For the global phosphoproteome and the pYome data the following p-site annotation was mapped: kinase motifs (phospho motifs; HPRD database) according to sequence window, known sites (PSP and UniProt database) and known kinasesubstrate relations (PSP database) according to sequence window and UniProt protein IDs. Each protein group entry was annotated according to UniProt ID by Pfam, KEGG, GO BF and UniProt Keywords. P-sites with a SILAC ratio $\leq-0.75$ or $\geq 0.75$ for the global phosphoproteome and $\leq-1$ or $\geq 1$ for the pYome at least one time point were considered as regulated. For the protein expression analysis protein SILAC ratios $\leq-0.75$ or $\geq 0.75$ were accounted as regulated. For the phosphoproteomic cluster analysis, z-scoring (rows, no grouping) and the hierarchical clustering algorithm (row clustering with Euclidean distance, average linkage, preprocess with k-means, number of clusters 300) implemented in Perseus were applied. Four row clusters were defined for the global phosphoproteome data, and two row clusters were defined for the pYome data.

\subsubsection{Functional annotation of $B C R$ signaling effectors}

Gene names of proteins with at least one regulated phosphorylation site in the DG75 BCR time course experiments were searched manually in the UniProt and the PSP database for functional information. Furthermore, the respective gene name was searched in combination with the terms 'B lymphocytes' and/or 'B cell lymphoma' in the PubMed database for relevant association which is not yet implemented in common databases.

\subsubsection{BCR stimulation time course in three distinct $B L$ cell lines}

The DG75/Daudi and the label-free FFM_BL1 primary cell line dataset were loaded from the respective Phospho(STY)Sites.txt table. 'Reverse' and 'contaminant' entries were filtered, sites with a localization probability $<0.75$ and were removed and the SILAC ratios (DG75 and Daudi) were logarithmized. The log2 of the LFQ intensities of label-free BL1 cells was calculated and sites with less than two quantitation value in the time course were removed. Missing data was 
imputed from a normal distribution with a width of 0.5 and a down shift 1.6 for each column separately. The log2 LFQ intensity values after 2 and 10 minutes of stimulation were normalized to the non-stimulated time point $(0 \mathrm{~min})$. P-site annotation was mapped as described above. For each protein group entry Reactome, Pfam, KEGG, GO BF, MF and CC and UniProt Keywords annotation was added according to UniProt identifier. The 31 amino acids long sequence motif was shortened, starting at amino acid position six and a total length of eleven amino acids. For the analysis of common sites, the FFM_BL1 dataset was matched to the DG75/Daudi dataset by the short sequence windows. Sites with less than two quantitation ratios in either cell line were removed. Z-scoring (rows, no grouping) and the hierarchical clustering algorithm (row clustering with Euclidean distance, average linkage, preprocess with k-means, number of clusters 300) implemented in Perseus were applied. Three clusters were defined and the most differential sites were manually extracted for further analysis.

\subsubsection{Antigen-dependent and -independent signaling in DG75}

'Reverse' and 'contaminant' entries were removed from the Phospho(STY)Sites.txt, as well as sites with a global localization probability lower than 0.75 . The normalized $H / L$ ratios of the CD79A knockdown were reversed, all ratios were logarithmized $(\log 2)$ and p-site annotation was mapped as described above. Each protein group entry was annotated according to UniProt ID by Reactome, Pfam, KEGG, GO BF, MF and CC and UniProt Keywords. Two separate BCR signaling groups were defined: tonic (BCR knockdown (BCR kd) and Syk inhibition (Syk-)) and antigen-dependent (BCR antigen stimulation time course)). For each group, sites with less than two quantitation ratios were removed. Z-scoring (rows, no grouping) and the hierarchical clustering algorithm (row clustering with Euclidean distance, average linkage, preprocess with k-means, number of clusters 300) implemented in Perseus were applied for heat map analysis. Three clusters were defined and the most differential sites were manually extracted for further analysis.

\subsubsection{BRAF interactome and inhibition}

For the BRAF interactome and BRAF inhibition analysis in DG75, proteinGroups.txt tables and Phospho(STY)Sites.txt (BRAF inhibition only) were filtered and processed as mentioned in 2.2.5.1. Proteins with normalized SILAC ratios $\leq-1.5$ and $\geq 1.5$ in the BRAF interactome analysis and proteins with SILAC ratios $\leq-1$ and $\geq 1$ after BRAF inhibition were considered to be 
specifically depleted or enriched, respectively. Regulation thresholds for the global phosphoproteome after BRAF inhibition were a normalized SILAC ratio $\leq-2$ or $\geq 2$.

\subsubsection{BTK inhibition in AML}

Downstream data analysis of MaxQuant results was done with Perseus v1.5.0.15 (GP) and v1.4.1.3 (pYome) (Cox and Mann, 2008). 'Reverse' and 'contaminant' (in the protein expression analyses also 'identified only by site' entries) were removed as well as phosphorylation sites with a global localization probability lower than 0.75 . Normalized SILAC ratios and the intensity values were logarithmized ( $\log 2$ and $\log 10$, respectively). For the global phosphoproteome and the pYome data, information about kinase motifs (phospho motifs; HPRD database) according to sequence window, known sites (PSP and UniProt database) and known kinase-substrate relations (PSP database) according to sequence window and UniProt protein IDs were added with Perseus. Each protein group entry was annotated according to UniProt ID by Pfam, KEGG, GO BF and UniProt Keywords. For the global phosphoproteome two out of three technical replicates were required to have a SILAC ratio. The median of at least two SILAC ratios was calculated. For the global phosphoproteome, phosphorylation sites with a SILAC ratio $<-0.5$ or $>0.5$ and for the pYome SILAC ratios $<-1$ or $>0.5$ were considered to be regulated and used for further analysis.

\subsubsection{Protein network analyses}

\subsubsection{Phosphorylation interaction network analysis in $\mathrm{BL}$}

Interaction network analysis of phosphorylated tyrosine $(\mathrm{pY})$ site containing proteins derived from the pYome dataset and phosphorylated serine and threonine (pS/pT) site containing proteins from the global phosphoproteome dataset were submitted to the STRING database (Version 9.1) for the retrieval of protein-protein interactions (Szklarczyk et al., 2014). High confidence (score 0.7), 'experiments' and 'databases' as active prediction parameters were required. Networks were visualized with Cytoscape (Version 3.2.0.), further adaptations and modifications were done with Illustrator 5.1 (Shannon et al., 2003).

\subsection{PhosphoPath analysis}

Additional network visualization of quantitative phosphoproteomic datasets were performed with the Cytoscape (Version 3.2.1) App PhosphoPath (Version 1.1). Protein-protein interaction information was retrieved from BioGRID and STRING databases, pathway information from 
WikiPathways and kinase-substrate information from PSP. The quantitative networks were prepared as described by Raaijmakers et al., 2015. The networks were expanded with kinases from PSP and proteins from BioGRID (Raaijmakers et al., 2015).

\subsubsection{BTK inhibition networks in AML}

All proteins with one or several phosphorylation sites considered to be regulated in the pYome experiment (normalized $p$-sites SILAC ratios on a log 2 scale $\leq-1$ or $\geq 0.5$ ) were used to generate a protein-protein interaction network based on the STRING database (Version 9.1). High confidence (score 0.7), 'experiments' and 'databases' were used as active prediction parameters. Visualization was performed with Cytoscape (Version 3.0.2) (Shannon et al., 2003).

\subsubsection{TCR signaling adapters}

Downstream data analysis of MaxQuant results was done with Perseus v1.5.0.15. SLP76 (LCP2) and GADS (GRAP2) p-sites were extracted from the Phospho(STY)Sites.txt. Normalized SILAC ratios and the intensity values were logarithmized ( $\log 2$ and $\log 10$, respectively). P-sites with less than three quantitation values for every TCR stimulation time point (1, 2, 5 and $10 \mathrm{~min})$ in four biological replicates and those sites with a global localization probability lower than 0.75 were removed. Information about kinase motifs (phospho motifs; HPRD database) according to sequence window, known sites (PSP and UniProt database) and known kinase-substrate relations (PSP database) according to sequence window and UniProt protein IDs were added. The median of at least three SILAC ratios was calculated with Perseus. Sites were manually grouped according to their phosphorylation behaviour and visualized with Excel.

\subsubsection{Manual spectra annotation}

MS or MS/MS spectra were extracted from the raw data with Xcalibur or the MaxQuant viewer (implemented in MaxQuant) and modified with Illustrator (v5.1) for better legibility.

\subsubsection{Arginine-to-proline conversion}

For the determination of potential arginine-to-proline conversion, the peptides.txt table was loaded in Perseus (any version). 'Reverse' and 'contaminant' entries were removed, nonnormalized SILAC ratios were logarithmized $(\log 2)$ and the density of the SILAC ratios against the proline content ( $P$ count) was calculated. SILAC ratios were plotted against the proline content and color-coded according to the density. 


\subsubsection{Kinobead analysis}

The list of identified proteins was filtered for kinases. Intensity values for 2, 5, 10 and 20 min of BCR stimulation were divided by the intensity value measured at 0 min (non-stimulated). Resulting ratios were logarithmized and kinases with values $\leq-1$ or $\geq+1$ were considered to have an altered binding behavior to the kinobeads after BCR stimulation and were considered for further investigations.

\subsubsection{Complementary analyses}

In the following, analysis methods other than LC-MS/MS measurement that were performed by collaborators are listed in Table 12 .

\subsubsection{RNA sequencing}

\subsubsection{Sample preparation and data analysis}

Pellets were thawed in RNAlater (Qiagen) for RNA stabilization and total RNA was extracted using the RNeasy Mini Kit (Qiagen) following the manufacturer's instruction for total RNA extraction from human cells. The RNA Integrity Number (RIN) was determined for all samples on an Agilent 2100 Bioanalyzer using the 'Eukaryote Total RNA Nano Chip' (Agilent). All RNA samples had a RNA integrity number (RIN) greater than 8 in order to provide good quality sequences. Sequencing libraries were prepared from the poly-A RNA fraction of $1 \mu \mathrm{g}$ total RNA using the TruSeq RNA Sample Preparation Kit according to manufacturer's instructions (Illumina). Paired-end sequencing was conducted on an Illumina HiSeq2000 with 100 bp per read. A mean of 63 million reads with a standard deviation of 12 million was generated for the 60 samples. Generated sequences were aligned to the RefSeq human transcriptome using the Burrows-Wheeler Alignment (bwa) and concordance between reads and the reference genome (raw 'hits') per transcript were merged gene-wise (Li and Durbin, 2009; Pruitt et al., 2013). Counts per gene were analyzed using DeSEQ describing gene expression as a generalized linear model including treatment (BCR vs CONTROL), a factorial time effect and the combined effect as full model and a model lacking the combined effect as reduced model (Anders and Huber, 2010). The resulting $p$-values (indicating a change over time depending on the treatment) were adjusted according to Benjamini-Hochberg (Benjamini and Hochberg, 1995). Heat maps were generated using the package 'gplots' for the statistical programming language R (R Core Team, 2014; Warnes et al., 2013). 


\subsection{Transcription factor enrichment analysis}

The transcription factor analysis was performed with the statistical computing environment $R$ (Version 3.0.2). The TRANSFAC database (Version 2014.1) was used to match human upstream transcription factors to differentially expressed target genes found in the transcriptomic analysis (Matys et al., 2005; R Core Team, 2014). Fisher's exact test was used for transcription factor enrichment analysis. The resulting $p$-values were corrected for multiple testing with the Benjamini-Hochberg method (Benjamini and Hochberg, 1995). The heatmap.2 function of the R package 'gplots' was used for visualization of the dynamic target gene counts of significantly enriched transcription factors (Warnes et al., 2013). For cluster analysis, distances were measured as 1 - Pearson's correlation coefficient. The row dendrogram was computed and ordered based on row means. 'Rgraphviz' R package was used for visualization of transcription factor target gene assignments (Hansen et al., 2010).

\subsubsection{Western blot and $\mathrm{Ca}^{2+}$ flux analysis}

Western blotting was performed as described in Oellerich et al., 2013. For $\mathrm{Ca}^{2+}$ flux analysis after BCR engagement, DG75 cells were loaded with the ratiometric $\mathrm{Ca}^{2+}$-chelator INDO-1-AM and subjected to flow cytometry. 


\section{Results}

Protein PTMs such as phosphorylation play an important role in cell signaling processes. As dysregulated signaling is often involved in diseases such as cancer, a large-scale analysis of phosphorylation sites ( $p$-sites) by tandem mass spectrometry was used to globally quantify the dynamic phosphoproteome in Burkitt's lymphoma (chapter 3.1), acute myeloid leukemia (chapter 3.2) and T cells (chapter 3.3) under various experimental conditions. The resulting large-scale datasets were analyzed in terms of global parameters. Furthermore, single interesting phosphorylation sites were additionally highlighted in a functional context based on literature search.

\subsection{BCR signaling in Burkitt's lymphoma}

BCR-dependent signaling mechanisms in malignant transformed BL cells are poorly understood. The experimental setup described in the following aimed to globally characterize the B-cell receptor dependent phosphorylation status in BL cells during a defined time course of BCR stimulation. BCR stimulation was achieved with antibodies directed against the receptor as described in 2.2.1.3.1 leading to BCR engagement on the cell surface similar to antigen binding (Cao et al., 2012; Kishimoto et al., 1977; Schwartz et al., 2010). The comprehensive characterization of BCR signaling in DG75 was done by additional complementary time-dependent approaches i.e. transcriptional analysis and kinobead affinity purification. The kinase BRAF that was assumed to be activated according to the phosphoproteomic results was further screened for its interaction partners and its influence on the global phosphoproteome level. Time-dependent phosphorylation changes after BCR receptor engagement was additionally investigated in two other genetically distinct BL cell lines (Daudi and a primary, patient derived cell line FFM_BL1, or BL1) and compared to DG75 phosphorylation dynamics (Table 9). In this study, experimental emphasis is made on DG75 because it carries the MYC-IGH translocation $\mathrm{t}(8: 14)(q 24 ; \mathrm{q} 32)$ variant that is most common among BL patients (Molyneux et al., 2012). Moreover, the cell line has functional BCR signaling and can be genetically manipulated by targeted knockout/-down approaches (Feederle et al., 2004). The BL cell line DG75 was established in 1975 from a metastatic pleural effusion of a male 10 year old patient suffering from primary abdominal Burkitt's lymphoma (Ben-Bassat 
et al., 1977). DG75 is derived from a sporadic, Epstein Barr virus (EBV)-negative Burkitt's lymphoma and expresses a surface BCR of the IgM isotype (Ben-Bassat et al., 1977).

Daudi is derived from an endemic BL with a MYC translocation and FFM_BL1 a primary, sporadic BL cell line that carries various MYC point mutations (Table 9) (Haluska et al., 1987; Klein et al., 1968). As the main part of the Burkitt's lymphoma project is based on DG75, details about Daudi and FFM_BL1 cells will be provided in the respective results section 3.1.13. As mentioned previously in 1.2.1, BL is mainly driven by tonic, antigen-independent BCR signaling (Refaeli et al., 2008; Schmitz et al., 2012). Tonic BCR signaling was also investigated on the phosphoproteome level by two experimental approaches that allowed analysing BCR antigenindependent signaling in DG75 as described in 3.1.14.

\subsubsection{Strategy for the phosphoproteomic analysis of BCR signaling}

As a first step, the functional BCR signaling output was verified by BCR stimulation with antiIgM antibodies mimicking antigen binding followed by visualization of global tyrosine phosphorylation by western blot (Figure 3.1). BCR engagement lead to a sustained increase in total tyrosine phosphorylation after 2 min of stimulation (second to fifth lane, Figure $3.1 \mathrm{~A}$ ) compared to the non-stimulated state (first lane, Figure $3.1 \mathrm{~A}$ ). B-cell activation was additionally visualized by intracellular $\mathrm{Ca}^{2+}$ release. $\mathrm{Ca}^{2+}$ levels peak at around $60 \mathrm{~s}$ after $\mathrm{BCR}$ engagement and slowly approximate ground state after $10 \mathrm{~min}$ (Figure $3.1 \mathrm{~B}$ ). In order to characterize global, time-resolved protein expression and phosphorylation events after BCR engagement, a double triplex SILAC experiment covering the BCR stimulation time points 2,5 , 10 and 20 min after the stimulus was designed (Figure $3.1 \mathrm{C}$ ). Global protein expression profiling of the before mentioned time points was done via SDS-PAGE separation and in-gel digest followed by LC-MS/MS analysis as described in 2.2.2.1. Phosphoproteomic characterization was achieved using two different approaches: First, a combination of strong cation exchange (SCX) and titanium dioxide chromatography $\left(\mathrm{TiO}_{2}\right)$, which will be referred in the following as global phosphoproteome (GP); Second, an antibody-based purification of phosphorylated tyrosine peptides, which will be referred to as pYome (both approaches are described in 2.2.2.2.1 and 2.2.2.2.2). 
A

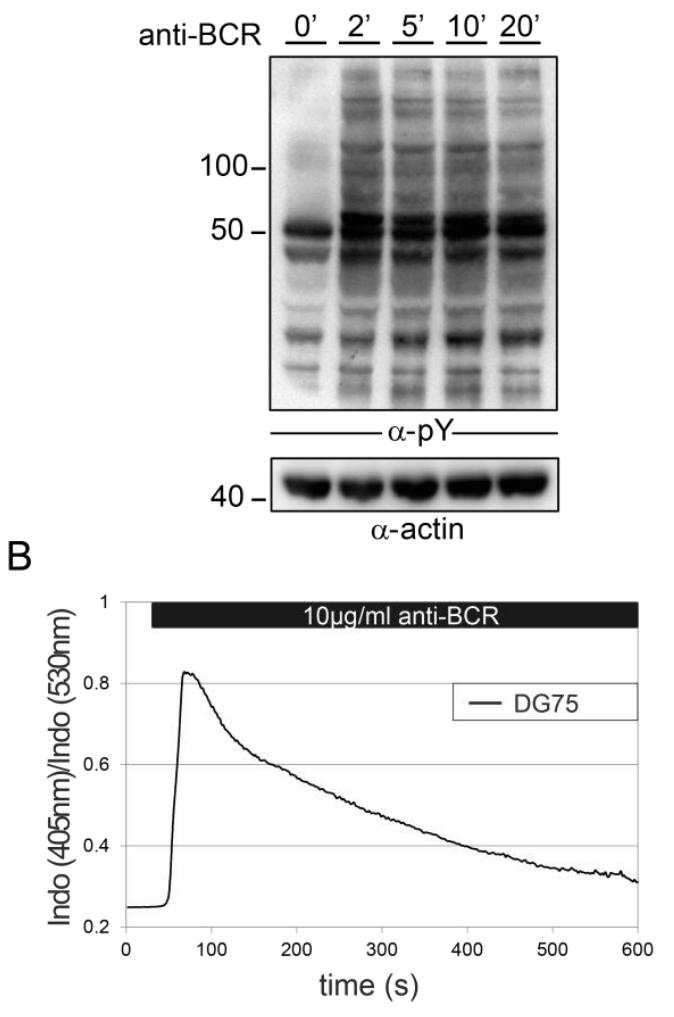

C

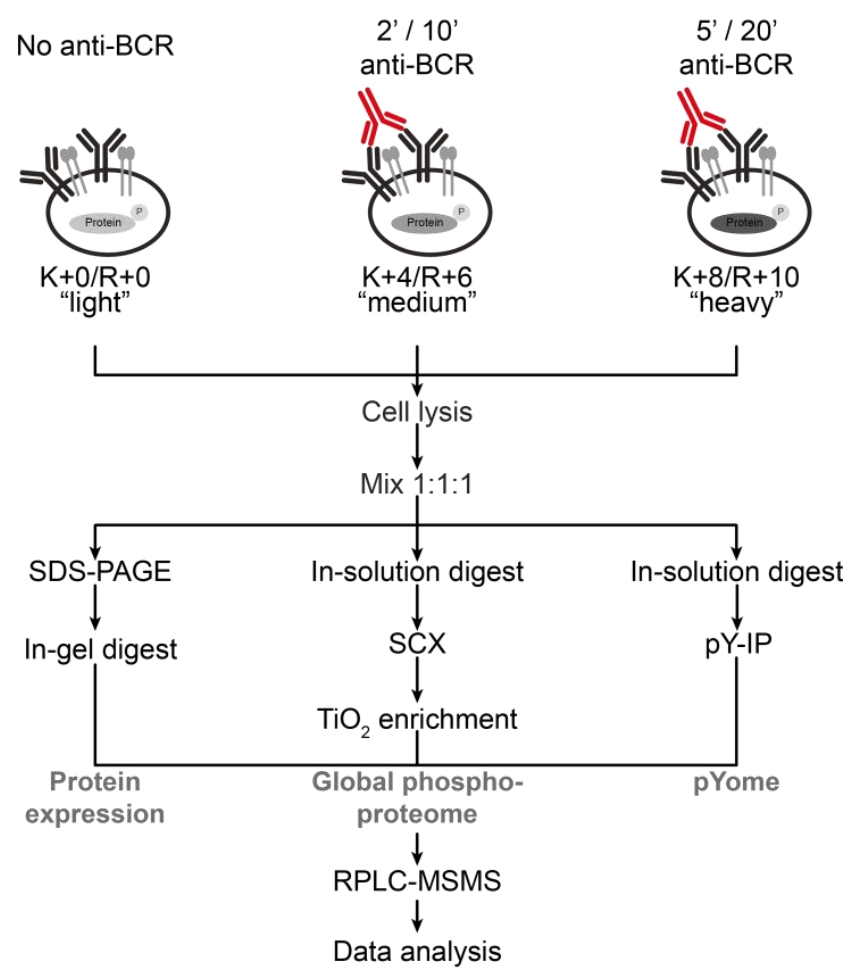

Figure 3.1: Phosphoproteomic BCR signaling analysis

(A) DG75 BL cells were left untreated or were BCR-stimulated for 2, 5, 10 and 20 min with anti-lgM antibodies, mimicking antigen stimulus. The western blot membrane was stained with an anti-pY antibody (4G10; upper panel) for visualization of total phosphotyrosine in the cells and for actin (lower panel) as loading control. (B) The intracellular $\mathrm{Ca}^{2+}$ concentration was measured until 10 min after BCR stimulation by flow cytometry. (C) Schematic representation of the experimental design of the double triplex SILAC approach for profiling protein expression and phosphorylation dynamics in resting and BCR stimulated DG75 cells. Cell lysates were mixed in equimolar amounts and digested by trypsin. For protein expression analysis, proteins were separated by SDS-PAGE, digested by trypsin and analyzed by LC-MS/MS. Phosphopeptides were enriched by a combination of $\mathrm{SCX}$ and $\mathrm{TiO}_{2}$ chromatography (global phosphoproteome; GP) or phosphotyrosine immunoprecipitation (pYome) and analyzed by LC-MS/MS. The pan anti-phosphotyrosine western blot and the flow cytometry-based $\mathrm{Ca}^{2+}$ flux assay shown in (A) and (B) were performed at the Department of Internal Medicine, Hematology/Oncology at the University Hospital Frankfurt/Main as part of the collaboration mentioned in Table 12.

\subsubsection{Protein expression levels}

Protein expression profiling for the BCR stimulation time course in DG75 was performed in order to confirm whether alterations in protein abundances through fast transcription, translation or protein degradation processes could influence correct phosphorylation site quantitation. Although a few proteins showed altered expression levels (proteins highlighted in red and with the gene name indicated; Figure 3.2) no general expression changes could be detected. Normalized SILAC ratios of the protein populations per time point of stimulation were normally distributed with the median value around zero (supplementary table S1). No previously known BCR signaling protein showed altered protein expression levels upon BCR 
stimulation. All proteins and quantitation ratios for all time points can be found in supplementary table S1.
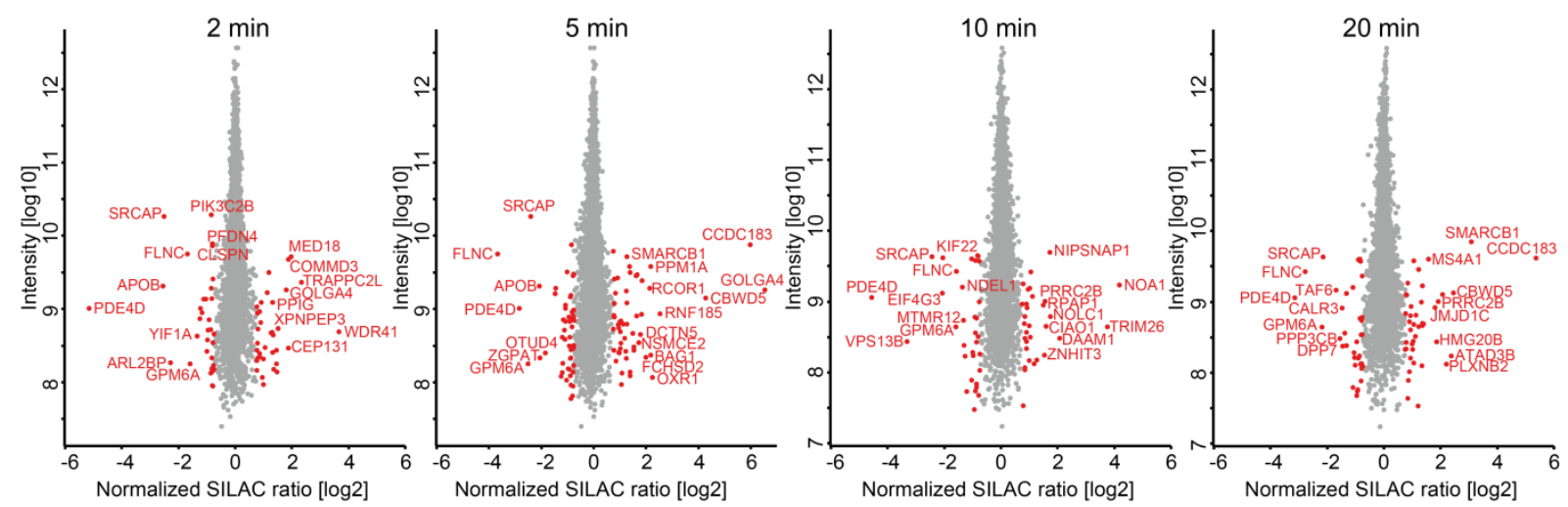

Figure 3.2: Protein expression after BCR stimulation in DG75

Scatter plots show the distribution of normalized SILAC ratios $(\log 2)$ of the protein groups versus their intensity $(\log 10)$ for 2 , 5, 10 and $20 \mathrm{~min}$ after BCR stimulation. Proteins with normalized SILAC ratios $\leq-0.75$ or $\geq 0.75$ (on a $\log 2$ scale) were highlighted in red. For better legibility, only gene names of proteins with the highest SILAC ratios were indicated. The complete protein list with quantitation values can be found in supplementary table S1.

\subsubsection{Identification, quantitation and regulation of phosphorylation sites}

The global $\mathrm{SCX} / \mathrm{TiO}_{2}$-based phosphopeptide enrichment lead to the identification of approximately 9000 individual phosphorylation sites (dashed outer circle, Figure 3.3 A). 4390 phosphorylation sites met the quality criteria of a localization probability $\geq 75 \%$, referred to as class I sites, and quantitation values in at least $50 \%$ biological replicates at single $B C R$ stimulation time points as described in 2.2.5.1 (outer circle, Figure 3.3 A). 730 of these sites were considered in the following to be regulated. This was defined by the normalized SILAC ratio $\leq-0.75$ or $\geq 0.75$ (on a $\log 2$ scale; inner circle, Figure $3.3 \mathrm{~A}$ ) for at least one time point. The distribution of the regulated $p$-sites derived from the GP was split between the three amino acid residues as follows: $85 \% \mathrm{pS}, 13 \% \mathrm{pT}$ and $2 \% \mathrm{pY}$. As mentioned earlier, tyrosine phosphorylation is on the one hand known to play a major role in signaling processes, but on the other hand is less abundant; therefore, antibody-based phosphotyrosine enrichment was additionally applied. Immunopurification with specific phosphotyrosine antibodies resulted in the identification of approximately 900 phosphorylation sites of which 336 fulfilled the quality criteria mentioned above (dashed outer circle and outer circle, Figure 3.3 B). 181 of p-sites were considered to be regulated with a normalized SILAC ratio $(\log 2) \leq-1$ or $\geq 1$ (inner circle, 
Figure 3.3 B). $82 \%$ of the regulated sites were phosphorylated tyrosines (blue grey pie, Figure 3.3 B). Different regulation thresholds were applied to the global phosphoproteome and the pYome respectively, to take the higher magnitude of phosphotyrosine site regulation for the pYome into account (Olsen et al., 2006; Satpathy et al., 2015) (reviewed in Hunter, 2014; Lim and Pawson, 2010). Normalized SILAC ratios of regulated $p$-sites of both experimental datasets were Z-scored and subjected to unsupervised hierarchical cluster analysis, respectively (Figure 3.3 C and D). Z-scoring transforms the time-dependent SILAC ratios of a p-site into standardized values with mean 0 and standard deviation 1 . P-sites from the global phosphoproteome analysis could be separated into four main clusters while those derived from the pYome analysis fell into two distinct clusters (Figure 3.3 C and D). The dominant cluster identified from the GP analysis represented immediate dephosphorylation of $\mathrm{p}$-sites after BCR engagement (Cluster 1, Figure 3.3 C), while in the pYome dataset, early upregulation followed by sustained phosphorylation after BCR engagement was characteristic (Cluster 1, Figure 3.3 D). Cluster 2 from the GP dataset showed immediate upregulation of $p$-sites after receptor stimulation whereas $p$-sites in cluster 3 showed a delayed, more gradual upregulation peaking after $5 \mathrm{~min}$ of $\mathrm{BCR}$ engagement (Figure $3.3 \mathrm{C}$ ). Gradual dephosphorylation of a few $p$-sites could be observed in both the GP and the pYome (Cluster 4, Figure 3.3 C; Cluster 2, Figure 3.3 D). Detailed information about all quantified p-sites and assigned cluster from the GP and the pYome analysis can be found in supplementary tables S2 and S3. 


\section{Results}

A Global phosphoproteome

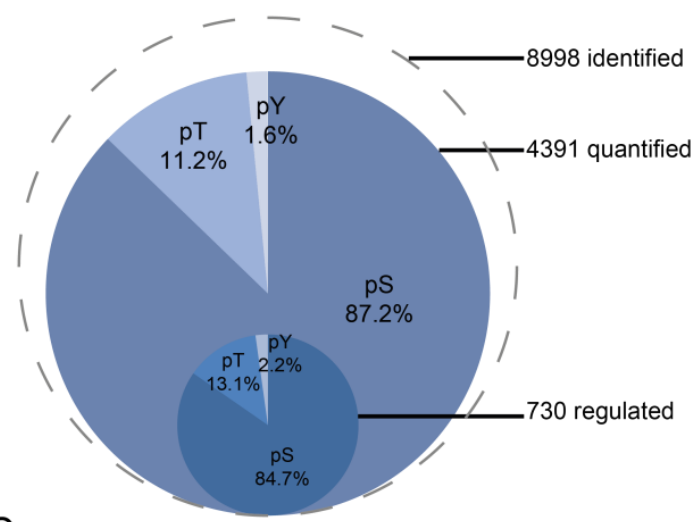

C

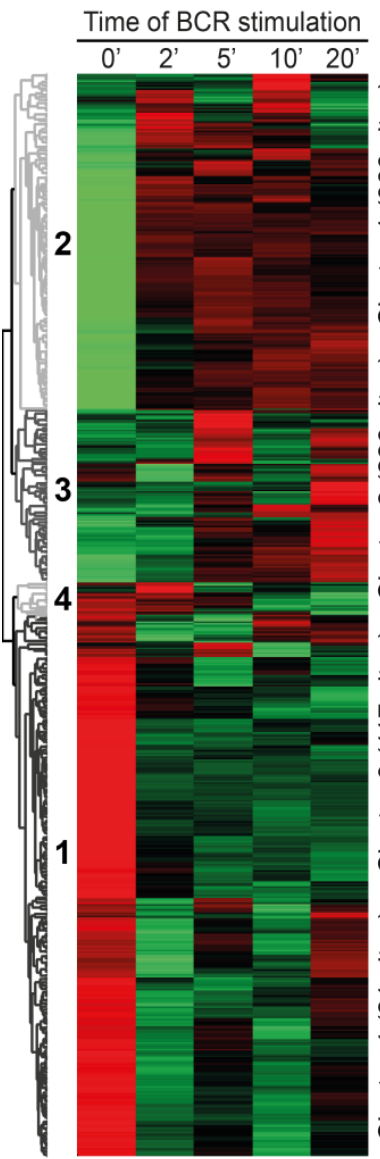

B

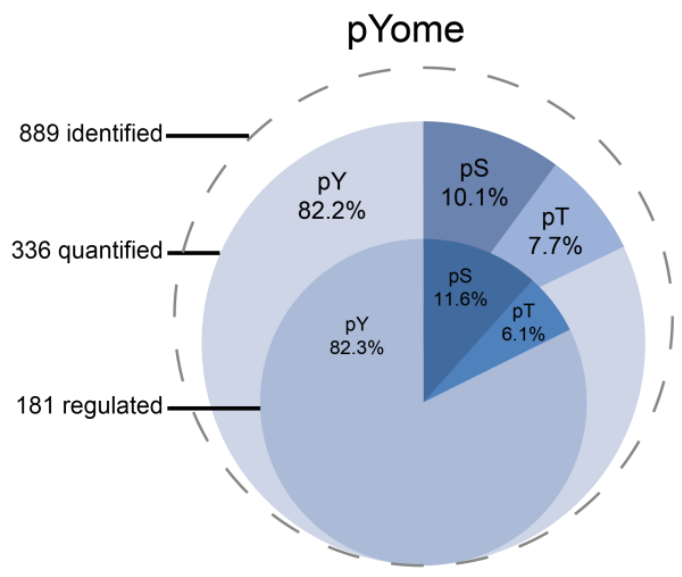

$\mathrm{D}$

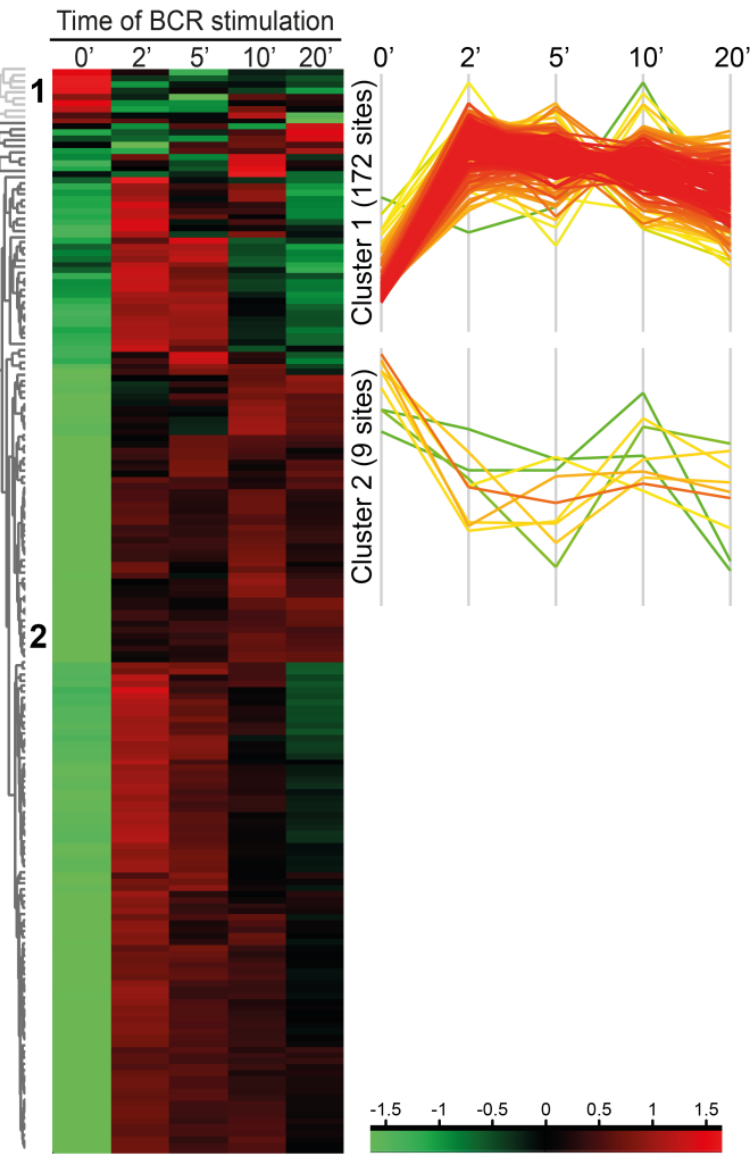

Figure 3.3: Phosphoproteome dynamics in DG75 after BCR stimulation

(A) and (B) Numbers of identified (dashed circle), quantified (outer circle) and regulated p-sites (inner circle) in the global phosphoproteome (A) and the pYome (B). The outer dashed circle represents non-proportionally the number of all $p$-sites identified across all BCR stimulation time points and biological replicates. The outer solid circle represents the number of quantified class I $p$-sites (localization probability $\geq 0.75$ ) at all stimulation durations in at least $50 \%$ biological replicates and the proportions of phosphoserine, -threonine and -tyrosine. The inner circle represents the relative abundance of quantified class I p-sites that were regulated in at least one time point during stimulation including the distribution of amino acids. The $\mathrm{p}$-site distribution between the three amino acid residues is shown as pie chart within the outer and the inner circle. (C) and (D) shows the hierarchical cluster analysis of all regulated p-sites (inner solid circles in A and B) of the global phosphoproteome (C) and the pYome (D) upon BCR stimulation, respectively. Values for each p-site (row) at all BCR stimulation durations (columns) are colored based on the $\log _{2}$-transformed and Z-scored normalized SILAC ratios (color scale at the right lower bottom). Line graphs next to the heat maps illustrate the phosphorylation dynamics upon BCR stimulation for each identified cluster. All quantified p-sites and assigned cluster shown in (C) and (D) can be found in supplementary tables S2 and S3. 


\subsubsection{Validation of MS-based quantitation}

To validate the quantitative, time-resolved proteomic and phosphoproteomic mass spectrometric results, the dynamics of selected phosphorylation sites of commonly known BCR effectors (BTK, MAPK1, MAPK3, SYK, BLNK, and CD79A/B) were visualized with western blot by using p-site- and protein-specific antibodies. The semi-quantitative western blot analysis of a BCR stimulated Burkitt's lymphoma cell line was compared to the MS-based quantitative results of DG75 (Figure 3.4 A and B). Selected sites are activation state-specific psites such as BTK Y551, SYK Y525, CBL Y674 or the dual phosphorylation TXY motif in MAPK1 and MAPK3 (ERK2 and ERK1) (Burack and Sturgill, 1997; Teckchandani et al., 2005; Tsang et al., 2008). For CD79A ( $\operatorname{Ig} \alpha)$ Y210, no site-specific antibody is available, therefore a p-site in close proximity, CD79A Y182 was visualized by western blot instead. Y182 is a valid substitute of Y210 as it lies outside the ITAM motif as well but flanks it from the $\mathrm{N}$-terminal site (Suthers and Young, 2014). BLNK and CD79A protein expression levels could not be monitored by MSbased proteome analysis. As CD79A forms a heterodimer with CD79B in the B-cell receptor, CD79B was visualized instead (Figure 3.4 A, lower panel). BLNK Y119 phosphorylation levels could be observed by MS-based phosphoproteomics only after 2 and 5 min of BCR stimulation (Figure 3.4 A, right lower panel). Protein expression levels did not undergo changes in any of the investigated proteins as can be seen both in MS-based quantitation and western blot analysis (Figure 3.4: bar diagrams in A and lower panels in B). Phosphorylation levels for all chosen p-sites increased immediately after 2 min of BCR stimulation and remained high until at least $10 \mathrm{~min}$ post-stimulation. In general, MS-based phosphorylation site dynamics of selected, regulated BCR-proximal effectors (BTK Y551, MAPK1 T185/Y187, MAPK3 T202/Y204, SYK Y525, CBL Y674, and BLNK Y119) could be reproduced by western blot (Figure 3.4: line diagrams in $A$ and upper panels in $B$ ), confirming that the quantitative MS-approach is a suitable method for the analysis of $p$-site dynamics. 
A

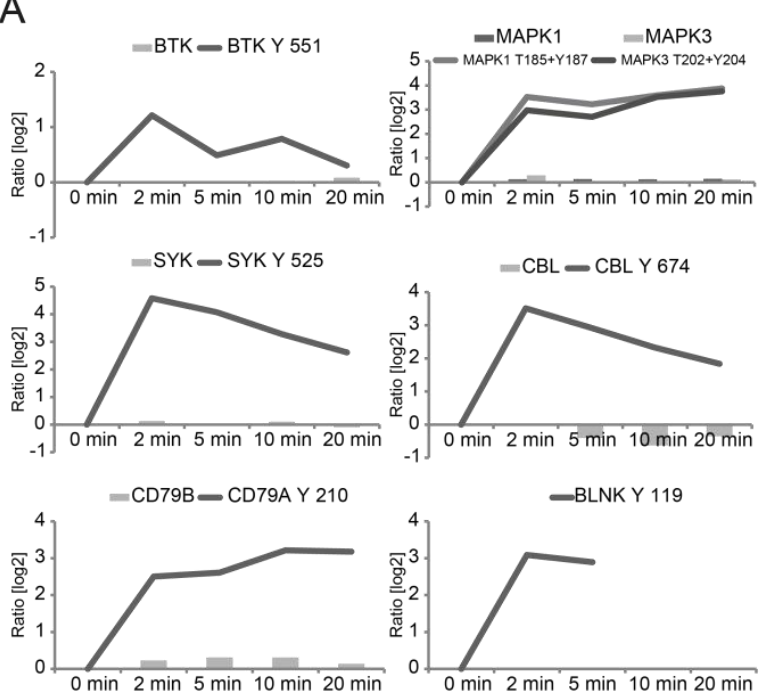

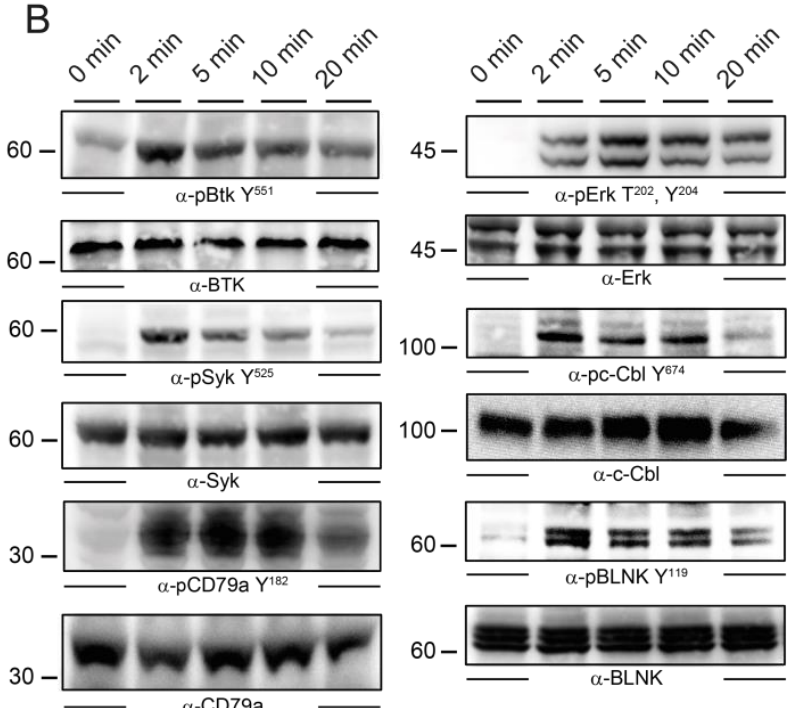

Figure 3.4: Validation of key phosphorylation sites in BCR signaling

(A) Mass spectrometric, SILAC-based quantitation ( $\log 2)$ of known BCR-dependent proteins (bar graphs) and selected p-sites (line graphs) of BTK, MAPK1 and 3 (ERK2 and 1), SYK, CBL, CD79A and BLNK (SLP65) that are representative for monitoring the activation state of the proteins. BLNK and CD79A protein expression levels could not be quantified. CD79B is shown instead of CD79A. (B) Phosphorylation dynamics and expression levels of the respective proteins at the indicated time points of BCR stimulation analyzed in (A) were visualized by immunoblotting with site- and protein-specific antibodies, respectively. Western blot analysis shown in (B) was performed at the Department of Internal Medicine, Hematology/Oncology at the University Hospital Frankfurt/Main as part of the collaboration mentioned in Table 12.

\subsubsection{Manual annotation of BCR signaling effectors}

Unbiased, large-scale data analysis harbours a wealth of information that need to be evaluated carefully in order to yield meaningful biological information. The global phosphoproteome and the pYome approach led in total to the identification of 857 unique regulated p-sites derived from 498 non-redundant proteins in DG75 cells upon BCR stimulation. Common annotation databases (e.g. KEGG, GO) often lag with the implementation of the most recent published information or do not provide enough biological detail. Therefore, an extensive literature (PubMed) and database search (UniProt and PhosphoSitePlus) was performed for the identified effector proteins in order to assign more detailed protein functions as described in 2.2.5.2. The three most prominent groups of $B C R$ effector functions are transcriptional regulation (13.3\%), cytoskeleton (9.2\%) and kinases (9.0\%) (Figure 3.5). The most dominant functional group of transcription regulators was found to be modified mostly by serine and threonine phosphorylation (blue and red proportion of the bar), while tyrosine phosphorylation (green proportion of the bar) targets mainly cytoskeleton, kinases and adapter proteins (Figure 3.5). 


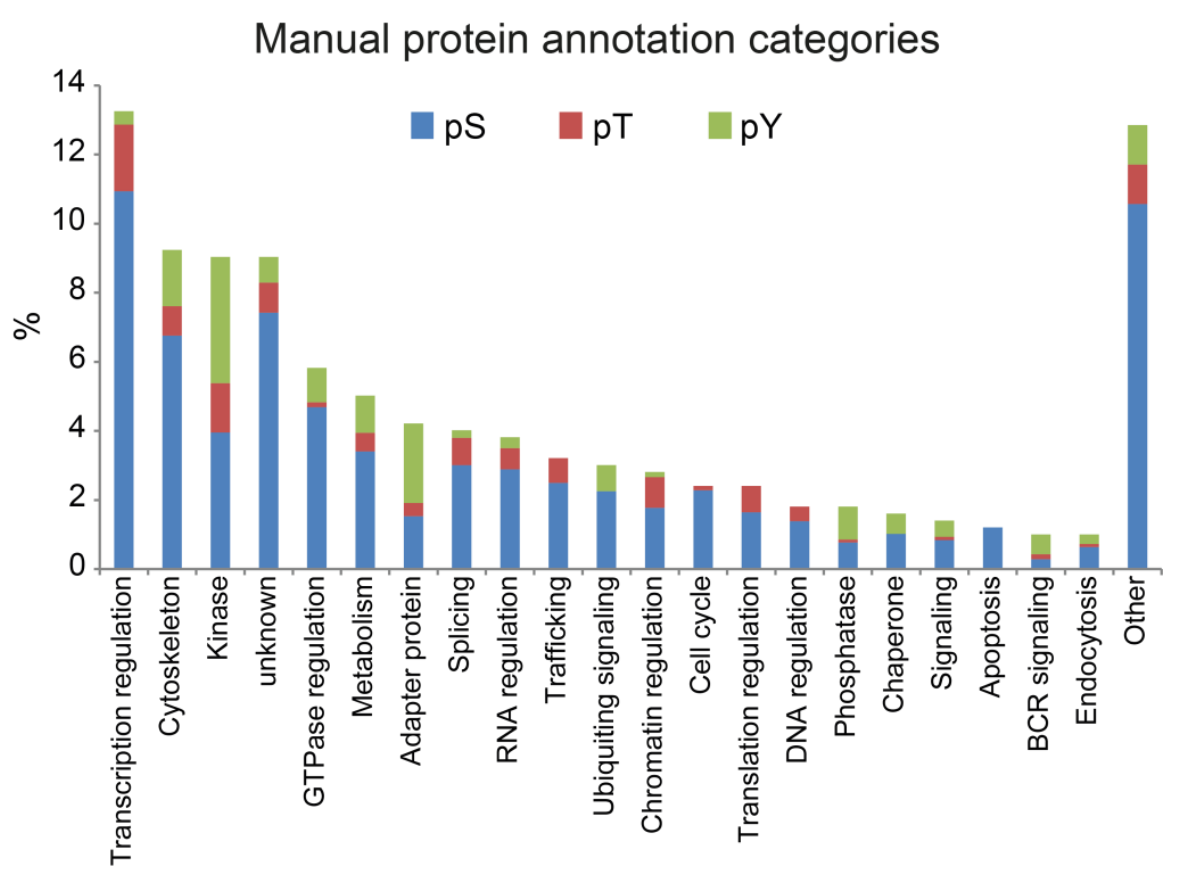

Figure 3.5: Manual annotation of regulated BCR effector proteins in DG75 cells

Distribution of protein effector functions responding to BCR stimulation according to manual protein function annotation. The proportions of phosphoserine, -threonine and -tyrosine for each category are color-coded within the bars. The manual protein annotation categories shown can be found in supplementary tables S2 and S3.

\subsubsection{Network analysis of BCR-dependent phosphorylation sites}

Tyrosine phosphorylation plays a major role in $\mathrm{BCR}$ receptor proximal signaling events (reviewed in Kurosaki et al., 2009). To examine how changes in tyrosine phosphorylation events are integrated into BCR downstream signaling, the identified effectors were visualized as a pY-signaling network based on the pYome data. Protein-protein interaction (PPI) information derived from the STRING database was retrieved for proteins identified with a regulated tyrosine-phosphorylation site in at least one BCR stimulation time point from the pYome analysis. Additionally, protein functions that were assigned by the manual annotation approach mentioned above (section 3.1.5) were added. Figure $3.6 \mathrm{~A}$ shows the generated pYome network, in which well-established BCR-proximal signaling effectors (highlighted in red), including Src kinases, SYK, BTK, PI3K, BLNK, the BCR co-receptor CD19 and MAP-kinases cluster highly connected in the center of the network. Other proteins responding to $\mathrm{BCR}$ stimulation are not yet linked to the central BCR signaling hub or are not yet described in the context of B-cell signaling and thus can be found connected in the periphery, respectively. Compared to the pYome network, the pS/pT interaction network derived from cluster $2 \mathrm{p}$ sites (described in section 3.1.3, Figure $3.3 \mathrm{C}$ ) of the global phosphoproteomic dataset is far 
less interconnected and does not have a central BCR signaling hub (Figure 3.6 B). Overall, also far fewer BCR effectors (highlighted in red) are known. In line with the manual protein function annotation shown in Figure $3.5 \mathrm{~B}$, the first and second most abundant categories 'Cytoskeleton' and 'Transcription regulation' contain the most, however yet noninterconnected effectors. 


\section{Results}

A

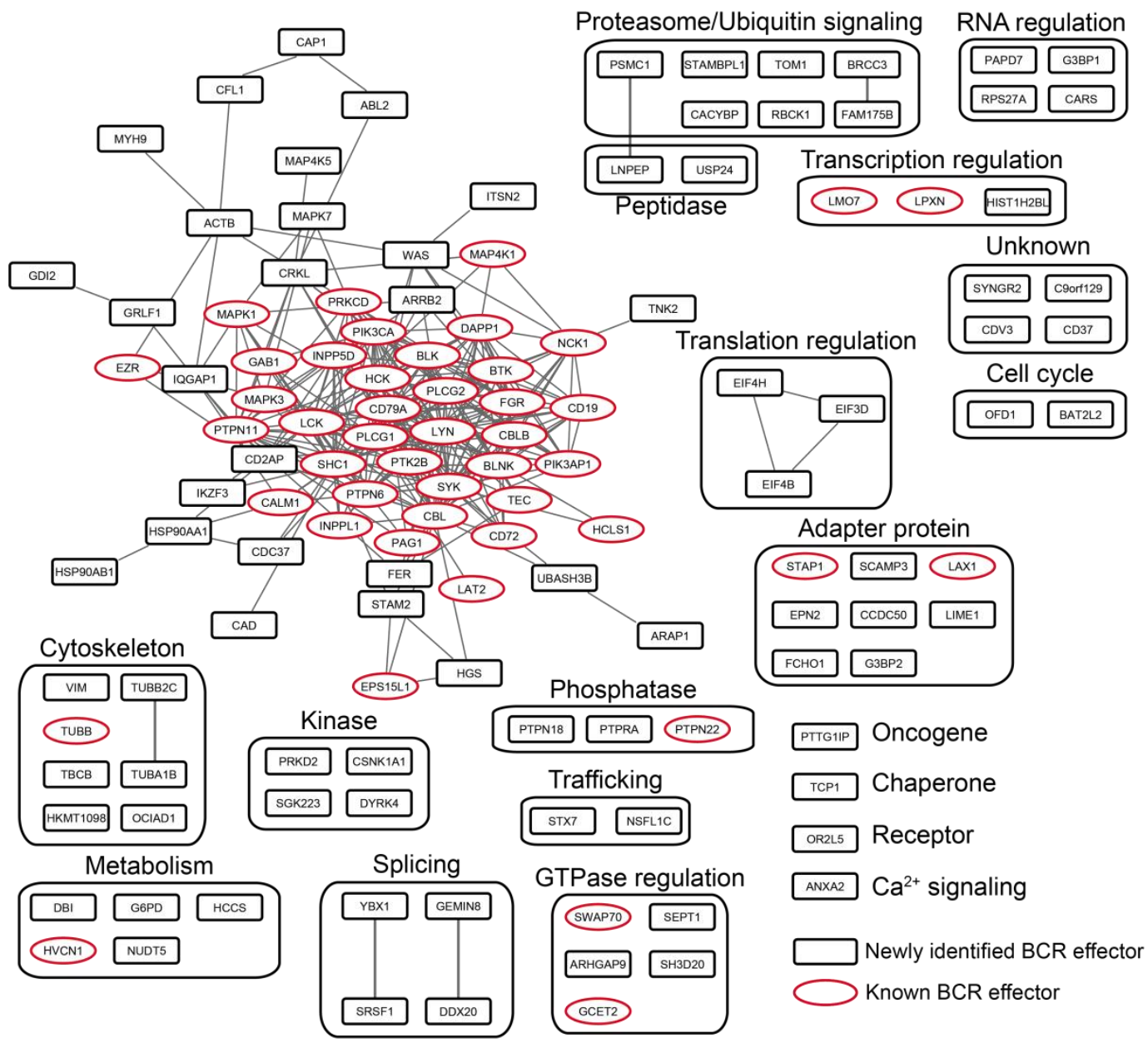

B

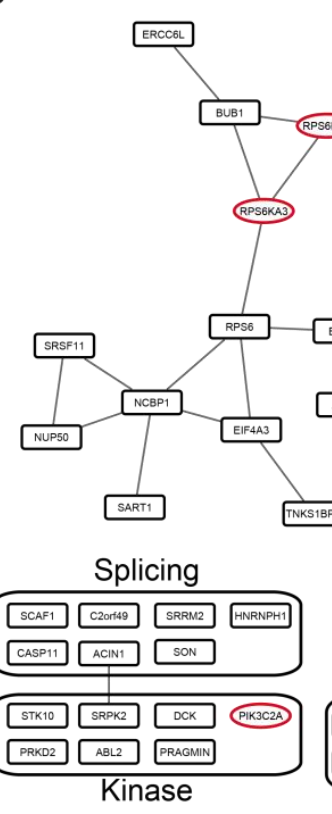

Adapter protein

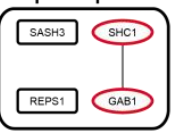

Cytoskeleton

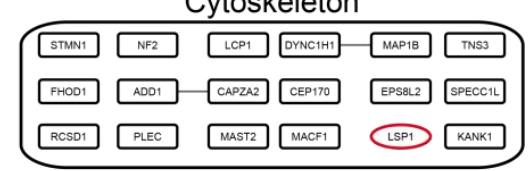
GTPase regulation Transcription regulation

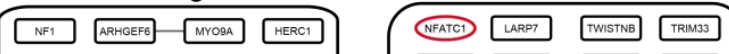

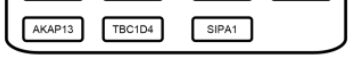

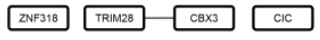
Translation (12) Ubiquitin signaling regulation LEPOP

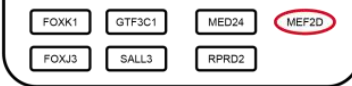

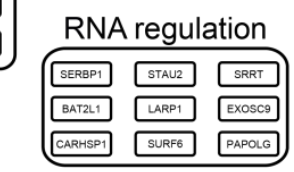

\section{Apoptosis} rexam zamm

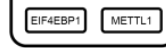

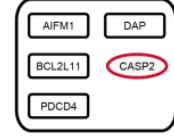
Metabaras

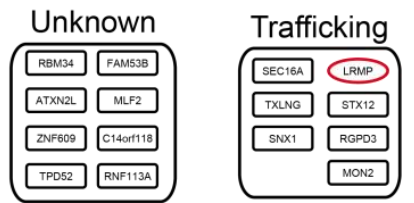

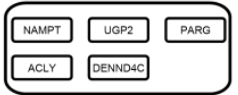
Phosphatase/Regulation

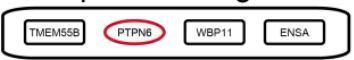

Kinase regulator DNA regulation Cell cycle Protein degradation

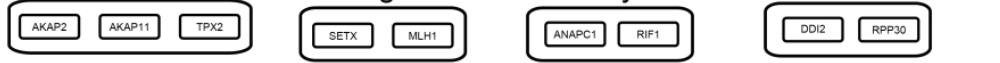
Nuclear transport Ribosome Chromatin regulation Chaperone

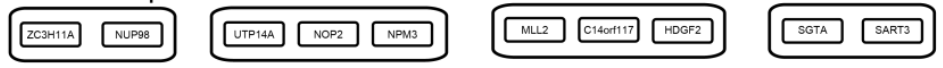
$\square$ Newly identified BCR effector $\bigcirc$ Known BCR effector enver Anti-apoptotic

ARFP Protein modification

(rait) Lymphocyte develoment

Receptor

sici2ald Ion channel

(sem BCR signaling

(rimal $\mathrm{Ca}^{2+}$ signaling

(corte Immune response

sxr Transporter

मrex+ra Endocytosis

Grofre Signaling

Endocytoris 
Figure 3.6: BCR effector protein-protein interaction networks

(A) Signaling network with all BCR effectors identified in the pYome dataset that have at least one regulated phosphotyrosine site during the time course. Sites quantified for 2 and 5 min upon BCR stimulation were also considered for this analysis. Proteins were grouped according to their enlisted protein-protein interaction (PPI) status in the STRING database. Red ellipses mark previously known BCR effectors, black rectangles depict those BCR effectors that have not been described in the B cell context before according to manual annotation. Proteins not engaged in the signaling network are categorized and grouped according to their manual annotated function. (B) Signaling network containing all pS and pT BCR effectors identified in Cluster 2 of the global phosphoproteome experiment (Figure 3.3 C). Grouping of proteins and color coding of the nodes is described in $(A)$.

\subsubsection{Transcriptional changes after $B C R$ signaling}

Binding of an extracellular antigen to the membrane-bound BCR leads, after PTM-mediated signaling through the cytosol, finally to changes in transcriptional regulation. This is also reflected by the previous finding that transcriptional regulators are the most abundant regulated functional group (Figure 3.5). Transcription can be regulated by various mechanisms at different cellular levels such as elevated expression levels of a transcription factor (TF), altered activity or cellular localization of a TF mediated by post-translational modification, the recruitment of cofactors, histone modifications or by interference of non-coding RNA (Lee and Young, 2013). To identify the influence of phosphorylation processes on transcriptome dynamics, large-scale transcriptome sequencing in resting and BCR-stimulated DG75 cells (10 min, $20 \mathrm{~min}, 1 \mathrm{~h}$ and $2 \mathrm{~h}$ ) was performed in collaboration with Ekkehard Schütz as described in Table 12, section 2.2.6.1, and shown in Figure 3.7 A. A total of 105 transcripts were identified as being significantly regulated within two hours after BCR stimulation (Figure 3.7 B; supplementary table S4). Several transcripts already showed elevated expression levels (FC > 1) after 10 min of BCR stimulation (e.g. FOSB, heat shock proteins, chemokines and EGR family proteins), while others such as the negative regulator of EGR, NAB2 reach their expression maximum after 2 hours (Kumbrink et al., 2005). Downregulated transcripts (FC $<-0.1$ ) can also be found already after $10 \mathrm{~min}$ of BCR stimulation (e.g. SEMA7A and LRRC32). PIM1, a serine/threonine kinase which regulates the stability of the oncogene MYC by phosphorylation, thereby regulating its transcriptional activity, is downregulated one hour after BCR stimulation (Zhang et al., 2008; Zippo et al., 2007).

To identify TFs responsible for BCR-induced transcriptional regulation, a transcription factor enrichment analysis using the TRANSFAC database was performed in collaboration with Tim Beißbarth as described in Table 12 and section 2.2.6.1. The TRANSFAC database contains information about the relation of transcription factors to their respective target genes (Matys 
and Kel, 2006). An over-representation analysis identified ten transcription factors that had a significant number of target genes identified as being differentially expressed in the transcriptome analysis (supplementary table S4). The counts of differential expressed targets of the mapped TFs showed a dynamic change as displayed in Figure 3.7 C. Early and transient transcriptional activity (10 and 20 min after BCR stimulation) for the transcription factors AP-1, HSF, ELK-1 and AhR, can be distinguished from a more sustained activation profile of NFAT-1, SRF and C-ETS. In contrast to that, IPF1 showed a late and slight increase of transcriptional activation. The bipartite graph expands the overrepresentation analysis by mapping the overrepresented transcription factors to their target genes in a time-resolved manner (Figure 3.7 D). In order to link the PTM-based signal transduction to transcriptional regulation after BCR engagement, the phosphoproteomic as well as the TF enrichment datasets were screened for known transcription regulators in BCR signaling as stored in the IPA database (Figure 3.7 E). Five of the ten enriched transcription factors were previously described to play a role in B-cell development and function: CREB, Jun/AP1, ELK-1, ETS-1 and NFAT (blue coloured nodes, Figure 3.7 E) (Bories et al., 1995). Regulated p-sites after all four BCR stimulation durations were found in JUNB/AP-1 (T255 and S259) and NFAT (S233, S359 in NFATC1; S236, T325, S326 in NFATC2; Figure 3.7 E). Both p-sites of JUNB/AP-1 were of particular interest as they are located within a phosphodegron, a motif involved in phosphorylation-dependent ubiquitination and subsequent proteasomal degradation of the target proteins (PérezBenavente et al., 2012). The potential induced degradation by phosphorylation represented by the strong increase in phosphorylation of the respective pS-sites is in line with the observation of the decreased transcription activity of JunB/AP-1 after $10 \mathrm{~min}$ (Figure 3.7 E and C). The phosphorylation sites NFATC1 S233 and NFATC2 S236 lie within SPXX repeat regions which lead in case of dephosphorylation to the nuclear import of the proteins and could possibly influence the DNA binding affinity (Macian, 2005). The influence of phosphorylation of S359 (NFATC1) and T325/S326 (NFATC2) on transcriptional activity has not yet been functionally described. BCL- 6 was not found to be enriched at the mRNA level, however BCL6 plays an important role in B cells. It is an inhibitory TF exclusively expressed in B cells, responsible for germinal center formation and antibody affinity maturation downstream of the RAF-MEK-ERK signaling axis (Schwartz et al., 2010). Ten phosphorylation sites of BCL-6 upon BCR stimulation were identified. S427 was identified as regulated whereas the other quantified sites did not show any changes in phosphorylation after BCR stimulation (Figure 3.7 
E). No phosphorylation changes could be monitored for BCL-6 S333 and S343 during the BCR stimulation time course (Figure 3.7 E). Phosphorylation of these two sites is described to lead to BCL-6 degradation (Niu et al., 1998). For the transcription factor TFII-I (GTF2) three out of six p-sites (S412, T413, S784 in isoform 1, corresponding to S371, T372 and S743 in isoform 2; supplementary table S4) were identified as being regulated after BCR stimulation (Figure 3.7 E). The serines at positions S412 and S784 were previously connected to enhanced transcriptional activity when phosphorylated (Casteel et al., 2002). The heat shock factor protein (HSF1) regulates genes in response to stress (Holmberg et al., 2002). HSF1 S303 is described in the literature as an inhibitory site when phosphorylated. It was identified to be dephosphorylated after BCR stimulation in this study while transcription factor activity peaked at 10 min after BCR stimulation (Figure 3.7 E) (Holmberg et al., 2002). All enriched transcripts with their respective mapped transcription factors and $p$-site dynamics of overrepresented transcription factors are listed in supplementary table S4. 


\section{Results}

A

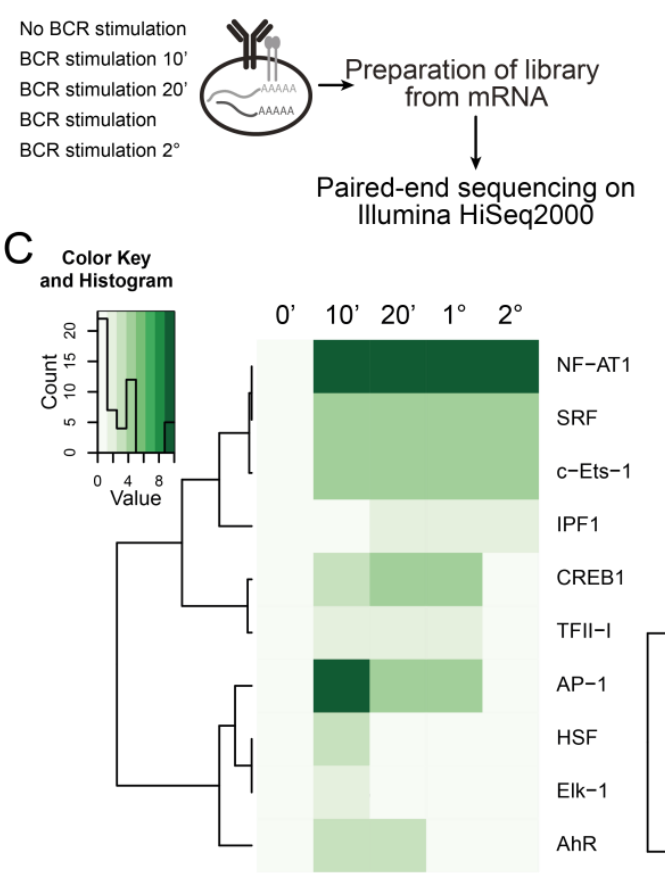

B

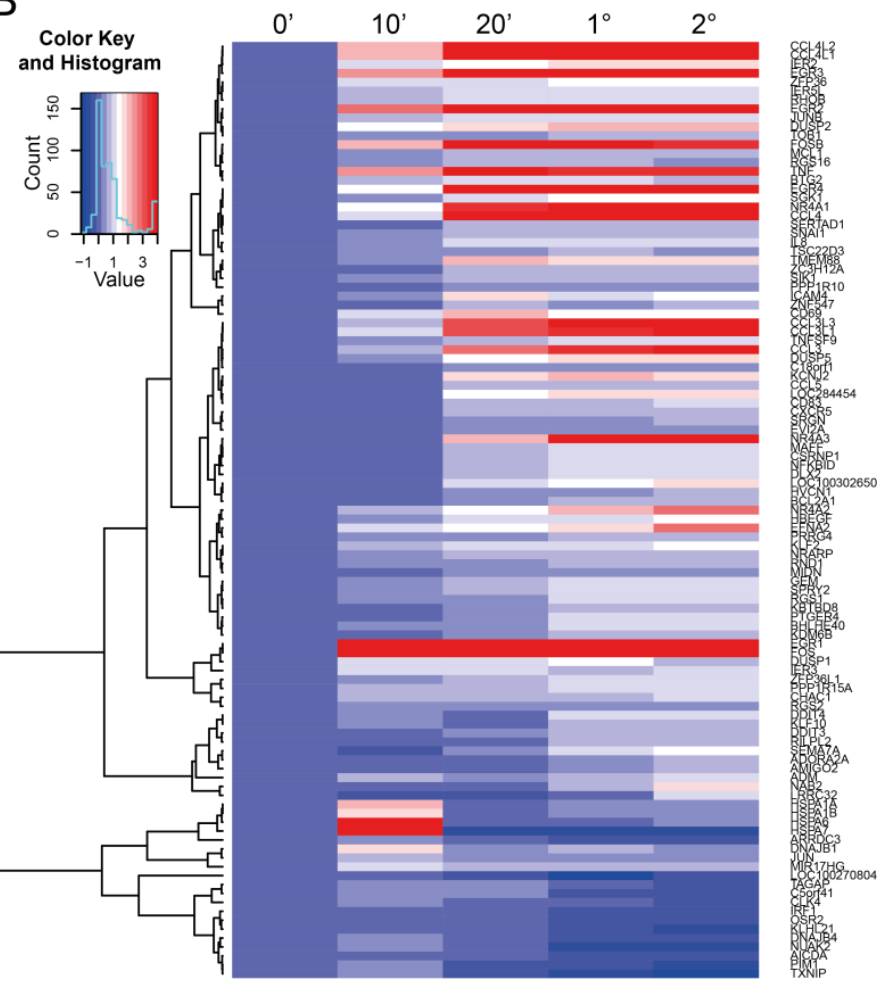

D

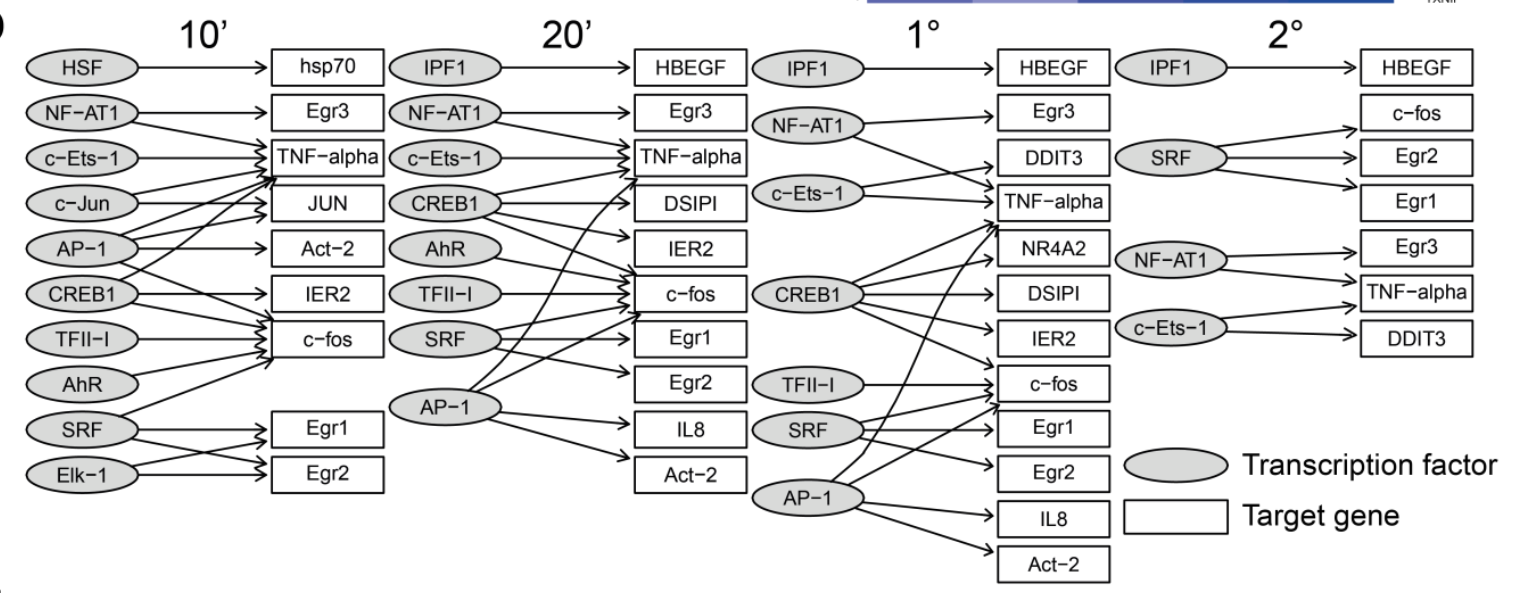

$\mathrm{E}$

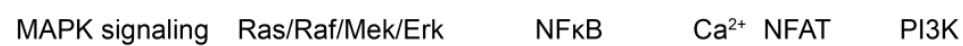

Signal transduction

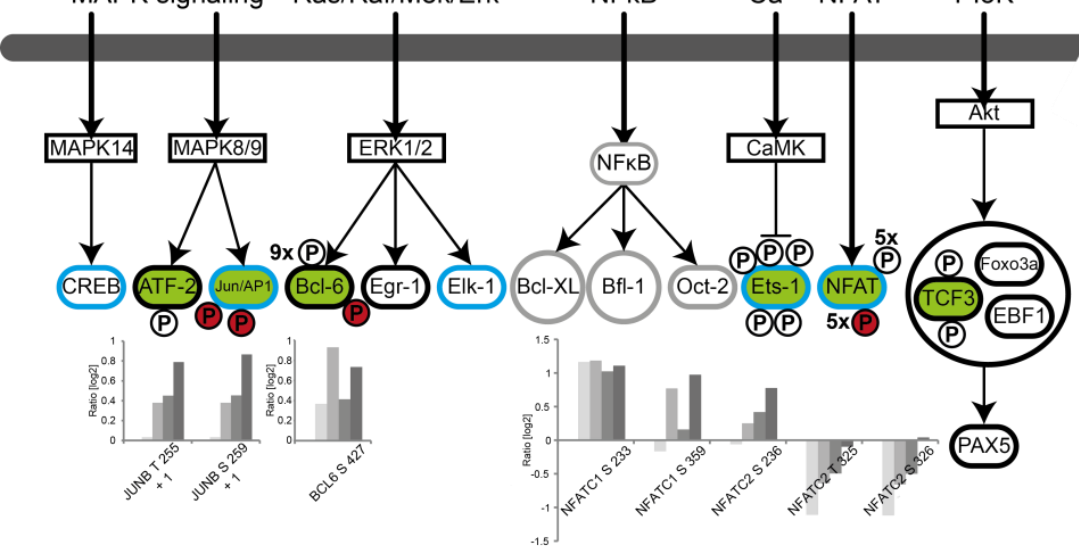

Transcription regulation
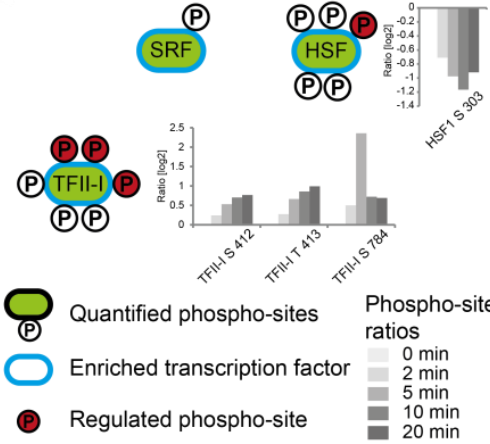

Figure 3.7: Time-dependent transcriptome analyses of BCR signaling

(A) Experimental workflow for the transcriptome sequencing of BCR stimulated DG75 cells. (B) Heat map illustrating the dynamics of 105 differentially expressed transcripts after $10 \mathrm{~min}, 20 \mathrm{~min}, 1 \mathrm{~h}$ and $2 \mathrm{~h}$ after BCR stimulation. (C) Heat map representing significantly enriched transcription factors for individual durations of BCR stimulation. Shown are the 
transcription factors assignable to target genes, which match the differentially expressed transcripts. (D) Bipartite graphs relating the transcription factors to their target genes for each BCR stimulation duration. Matching of the identified, differentially expressed transcripts to a transcription factor was done with the TRANSFAC database. (E) Enriched transcription factors (from C) set in context to database knowledge about BCR signaling using Ingenuity pathway analysis (left side). Enriched transcription factors that are not yet linked to BCR signaling, SRF, HSF and TFII-I (GTF2), are located in the right part of the scheme. Transcription factors responsible for the observed transcriptomic changes are marked with a blue border. Transcription factors with a green fill indicate $p$-sites that were identified in the DG75 global phosphoproteomic experiment. Quantifiable phosphorylation sites for these transcription factors are marked with ' $P$ '. A red fill indicates regulation and the corresponding quantified phosphorylation profiles are shown in the respective bar diagrams below. All regulated transcripts shown in (B), their respective transcription factors and the $p$-sites of overrepresented transcription factors shown in (C) and (D) are listed in supplementary table S4. RNA sequencing shown in (A) was performed by Chronix Biomedical, San Jose/Göttingen. The following data analysis shown in (B)-(D) was performed at the Institute of Medical Statistics, University Medical Center Göttingen as part of the collaboration mentioned in Table 12.

\subsubsection{Kinobead analysis}

Kinases are of major importance in BCR signal transduction as they are the mediators of phosphorylation on serine, threonine or tyrosine residues (reviewed in Kurosaki et al., 2009). Moreover, kinases were found to be one of the predominant protein families with regulated p-sites (Figure 3.5). Since small-molecule inhibitors can selectively target kinases, they became of particular interest for therapeutic strategies. In order to obtain more experimental evidence for active kinases, kinases were affinity purified from DG75 whole cell lysates with non-selective, commercially available kinase inhibitors immobilized on beads, namely kinobeads in collaboration with Bernhard Küster as described in Table 12 and section 2.2.5.11 (Bantscheff et al., 2007). The kinobead assay was performed for the time points after BCR stimulation defined for the previously described phosphoproteomic analysis i.e. 2, 5, 10 and 20 min (Bantscheff et al., 2007). The bound kinases after each time point were relatively quantified compared to the non-stimulated ground state with a label-free approach by mass spectrometry as described in 2.2.5.11 and Bantscheff et al., 2007. In total, 211 kinases could be quantified over the BCR stimulation time course of which three showed label-free quantitation values $\leq-1$ and twelve showed values $\geq+1$ on a log2 scale for at least one time point (supplementary table S5). It can be excluded from the protein expression analysis shown in 3.1.2. that increased binding to the kinobeads at very early time points after BCR stimulation is due to changes in kinase protein expression levels. Since kinase-to-bead binding is not necessarily associated with kinase activity, phosphorylation sites were used as an additional indicator to monitor kinase activity (Ruprecht et al., 2015). The p-site dynamics of potentially activated kinases as determined by the quantitative phosphorylation analysis shown in 3.1.3 were compared to their kinobead binding profiles, and additionally, set in a functional context (Figure 3.8). 
A

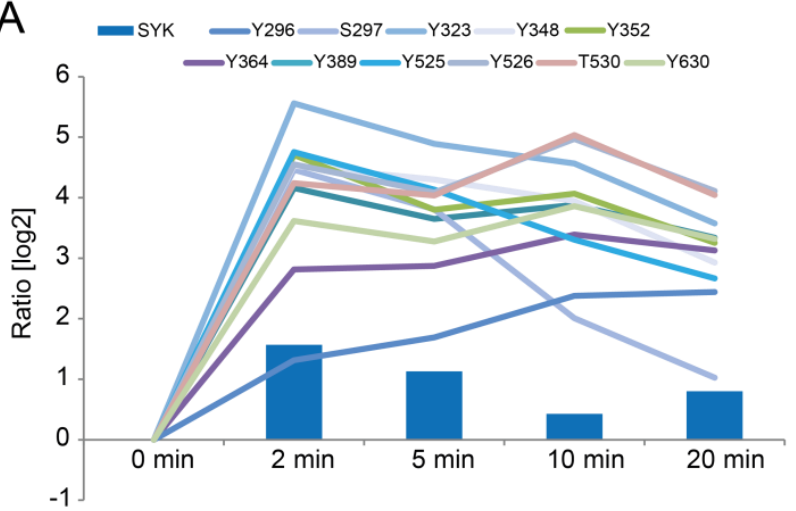

C

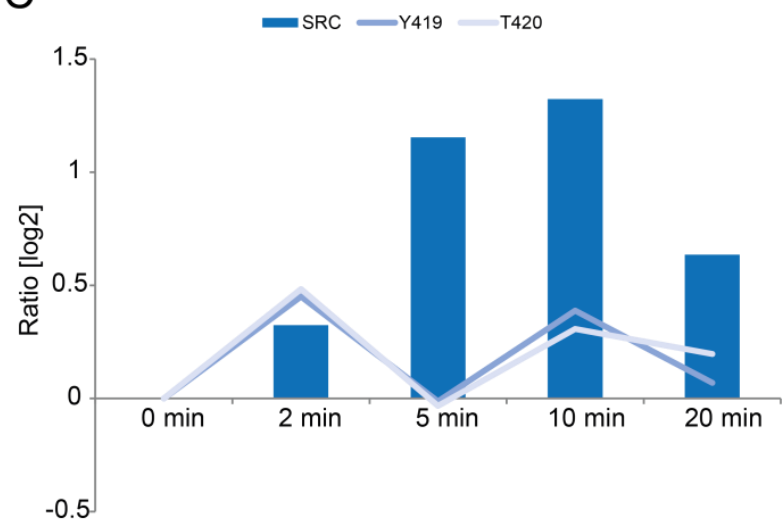

$\mathrm{B}=\mathrm{RPS6KA1}=\mathrm{RPS6KA3}=\mathrm{RPS6KA4}=\mathrm{RPS6KB1}$
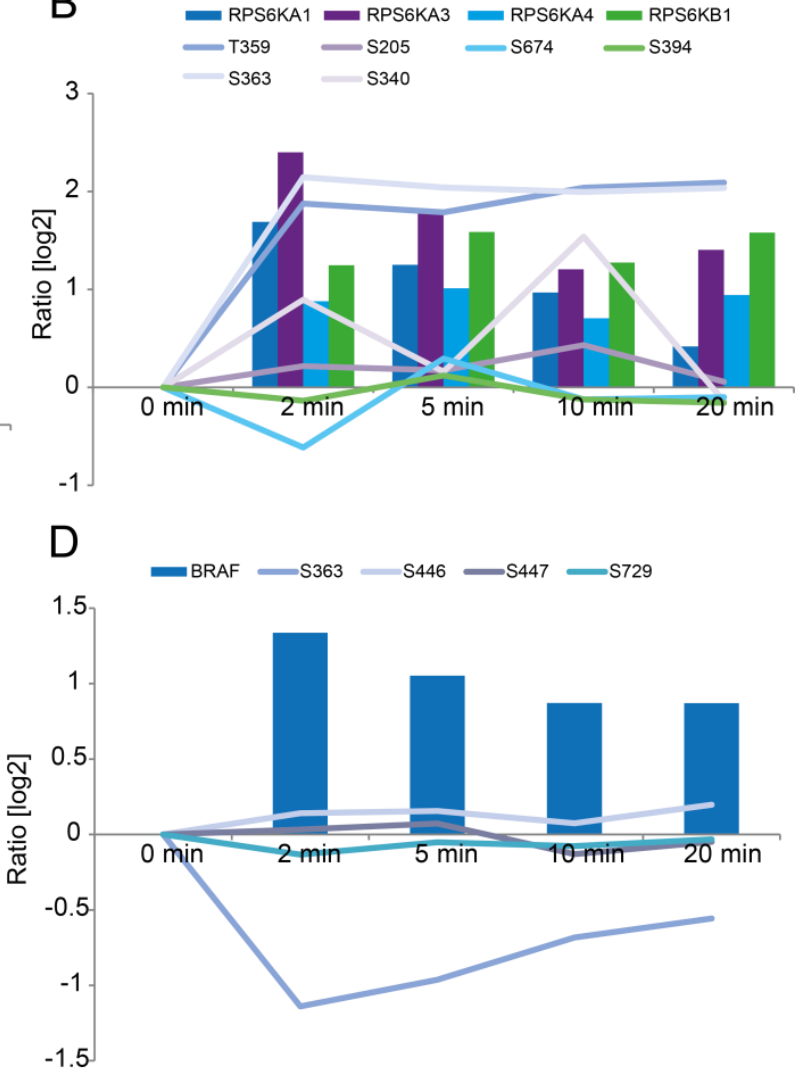

Figure 3.8: Kinase activation pattern in DG75 cells

(A-D) Bar diagrams showing kinases with a ratio $\geq 1$ or $\leq-1$ (on a log2 scale) in DG75 cells as revealed by a label-free kinobeadbased proteomic analysis and with quantifiable $p$-sites from the phosphoproteomic approach upon indicated durations of BCR stimulation. Line graphs represent indicated phosphorylation sites on the respective kinase (A) SYK (B) RSK family kinases (C) SRC and (D) BRAF, identified from the global phosphoproteome or the pYome dataset. All kinases with an altered affinity to kinobeads after BCR stimulation are listed in supplementary table S5. The kinobead assay and following data analysis was performed at the Chair of Proteomics and Bioanalytics at the Technical University München, Freising, as part of the collaboration mentioned in Table 12.

For seven kinases with an altered binding behaviour, $p$-sites could be identified from both, the GP or the pYome experimental data: SYK, four members of the ribosomal S6 kinase (RSK) family, SRC and BRAF. The time-resolved kinase binding profiles after BCR stimulation are shown in Figure 3.8 A-D. For SYK, 11 p-sites with a full phosphorylation profile could be quantified. The observed dynamics in the phosphorylation of sites (Figure 3.8 A) are located in kinase activity-regulating regions within the interdomain B (aa positions 260-370) or the kinase domain (aa positions 371-631). The correlation of the phosphorylation status of specific sites and the activation status of the kinase has been extensively described (Tsang et al., 2008). All SYK p-sites detected closely resemble the time profile for SYK binding to the kinobeads. For the four RSK family members RPS6KA1, RPS6KA3, RPS6KA4 and RPS6KB1, six p-sites could be 
quantified (Figure 3.8 B). A few were already associated with enhanced enzymatic activity e.g. T359 and S363 in RPS6KA1, S227 and S369 in RPS6KA3 and S447 in RPS6KB1 (Lekmine et al., 2003; Merienne et al., 2000; Wu et al., 2013). The phosphorylation of both sites in RPS6KA1 followed the kinobead-kinase binding profile and therefore could reflect kinase activity. The adjacent phosphorylation sites Y419 and T420 on the non-receptor tyrosine kinase SRC showed a similar fluctuating profile with phosphorylation maxima at 2 and $10 \mathrm{~min}$, respectively (Figure $3.8 \mathrm{C}$ ). In contrast to that, kinase-to-bead binding increased until $10 \mathrm{~min}$ of BCR stimulation and declined afterwards. The BRAF phosphorylation sites S446, S447 and S729 were not found to be regulated during the time course of stimulation although S446 and S729 are described as influencing kinase activity (Figure 3.8 D) (MacNicol et al., 2000; Tran et al., 2005). BRAF S363 was found to be dephosphorylated immediately after BCR stimulation and became slightly phosphorylated during the time course of stimulation, therefore inversely correlating with kinase-to-bead affinity (Figure 3.8 D). In the context of lymphoma, especially Burkitt's lymphoma, wild type BRAF is described rather than mutated, constitutively activated BRAF (V600E) (Cozzi et al., 2012; Schmitz et al., 2012; Spender and Inman, 2014). Several BRAF V600E inhibitors are available and BRAF inhibition is an approved treatment for melanoma patients (Flaherty et al., 2010). This made BRAF an interesting candidate for further analyses as described in the following.

BRAF S363 is the first of three consecutive serines within the peptide: pSSSAPNVHINTIEPVNIDDLIR (aa positions 363-386 in isoform 1). They comprise the Cterminal part of a generalized AKT consensus motif RXRXXS, where the two random amino acids represented by $X$ prior to the terminal serine (S365) are replaced by serines (S363 und S364) (Schwartz and Gygi, 2005). S365 (in the following references referred to as S364) is described as an inhibitory phosphorylation site with AKT as upstream kinase (Cheung et al., 2008; Guan et al., 2000; Zhang and Guan, 2001; Zimmermann and Moelling, 1999). It is not entirely clear if the upstream kinase AKT can exclusively target S365 or if rather the phosphorylation is distributed stochastically between the three serine residues. Furthermore, in previous studies the phosphorylation-mediated inhibition of BRAF was investigated with site-specific antibodies by western blot (Cheung et al., 2008; Guan et al., 2000; Zhang and Guan, 2001). It is also not entirely clear if these antibodies can selectively distinguish between the three potential site isomers pSSS, SpSS or SSpS. However, increased binding to the immobilized kinase inhibitors in combination with dephosphorylation of BRAF S363, adjacent 
to the previously described inhibitory p-site S365 within the AKT consensus motif hinted towards BRAF activation. For the matter of interest and further potential functional studies, the exact position of phosphorylation was determined by manual spectra annotation of the peptide (Figure 3.9). As mentioned previously, unambiguous site localization is not trivial, especially if the potential modifiable amino acids are in close proximity and the fragment ion series is not complete (section 1.3.8). The spectrum shown in Figure 3.9 A provides evidence for assigning the $p$-site unambiguously to the first serine $\mathbf{S 3 6 3}$, corresponding to a localization probability of $75.9 \%$ (supplementary table S2). Other spectra derived from the singly phosphorylated peptide did not provide unambiguous information about the exact phosphorylation site localization within the three $\mathrm{N}$-terminal serines (Figure $3.9 \mathrm{~B}$ and $\mathrm{C}$ ).

A
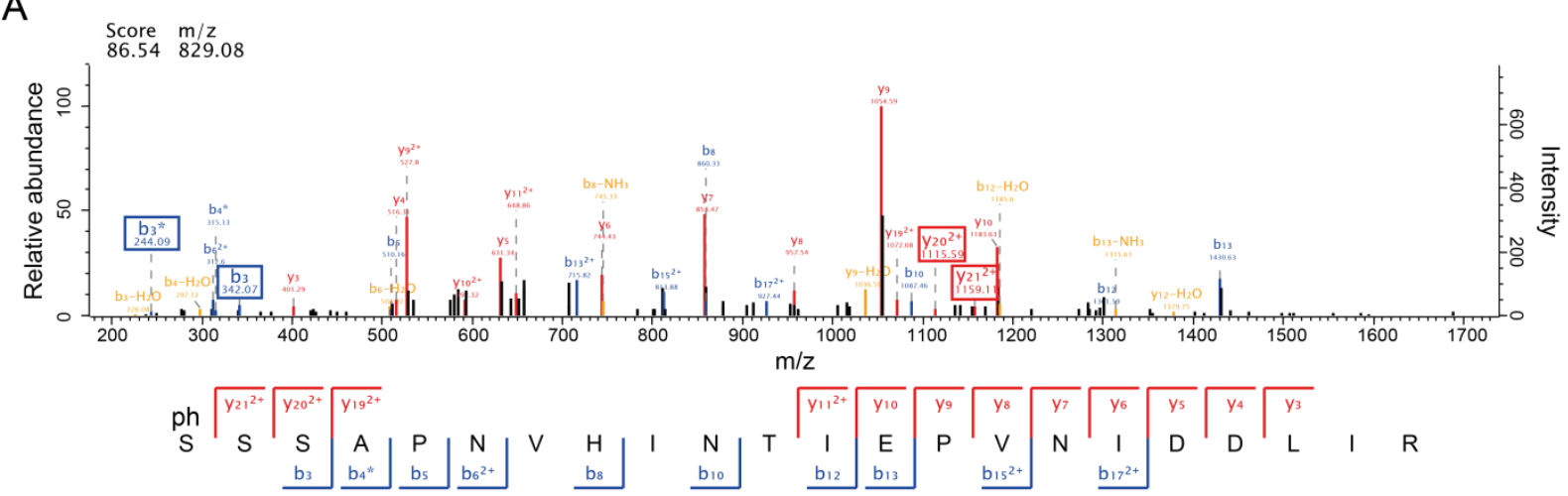

B
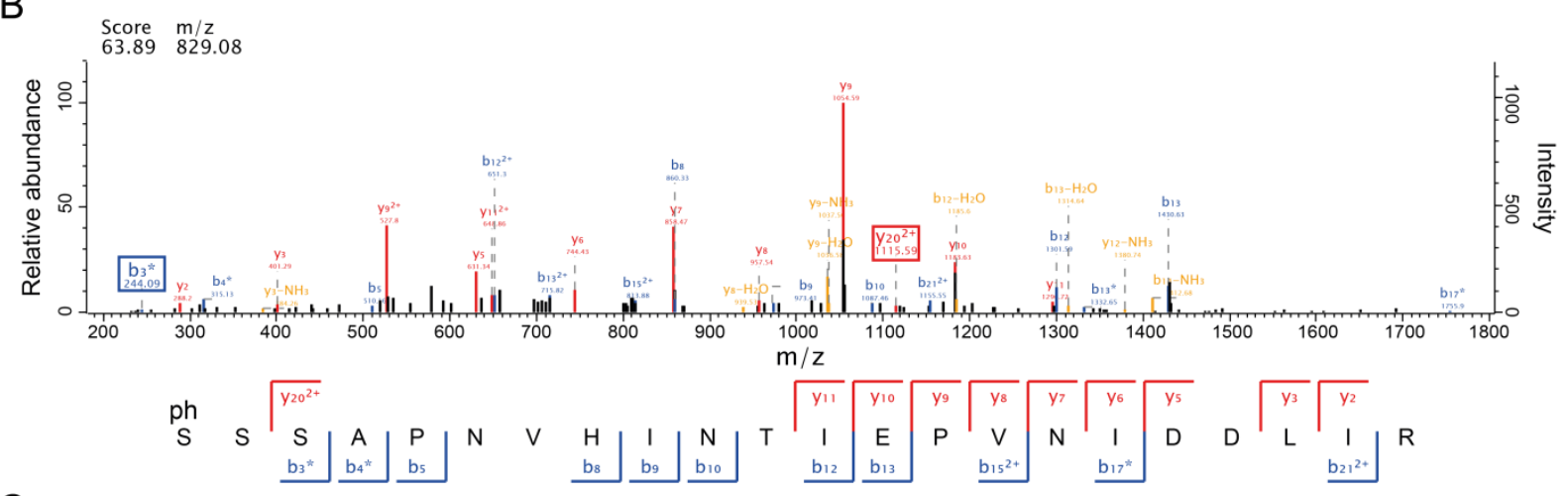

C

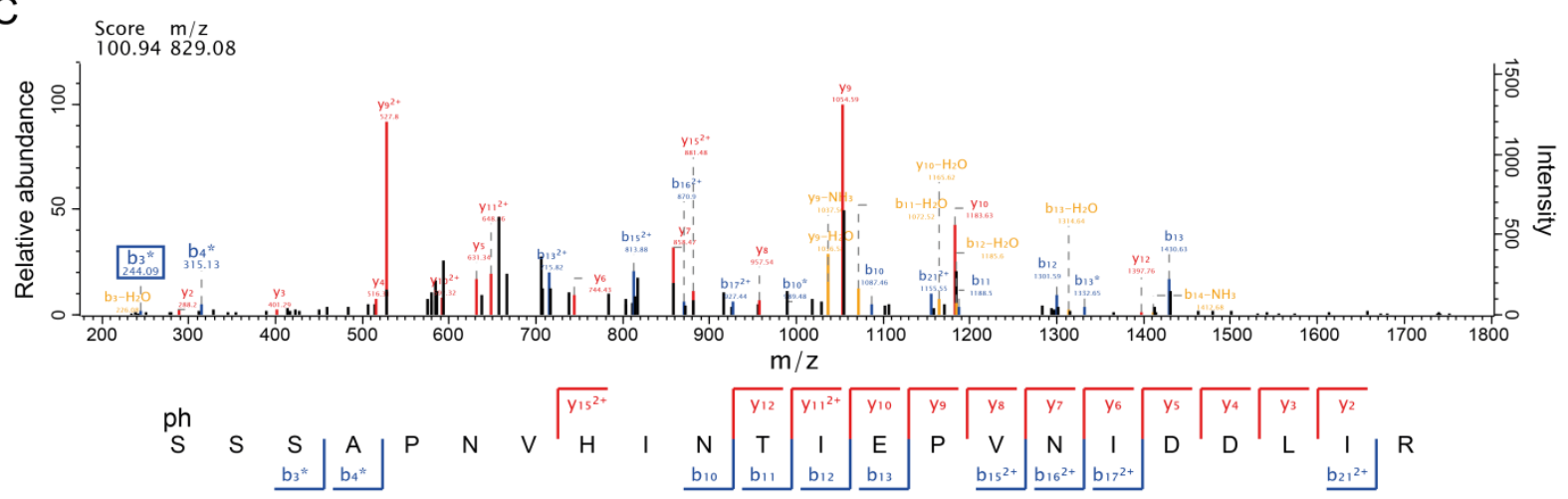


Ion trap CID MS/MS spectrum of the tryptic peptide pSSSAPNVHINTIEPVNIDDLIR (aa positons $363-384$ ) derived from BRAF. Neutral loss of $\mathrm{H}_{3} \mathrm{PO}_{4}$ (phosphoric acid) is indicated by an asterisk (*). B-ions are colored in blue, $\mathrm{y}$-ions in red and fragments with a neutral loss of $\mathrm{H}_{2} \mathrm{O}$ (water) and $\mathrm{NH}_{3}$ (ammonia) are colored in yellow. The $\mathrm{m} / z$ values are indicated below, the charge state $(z)$ is superscripted at the respective ions. The left $y$-axis shows the relative abundance of each fragment, the right $y$-axis the fragment ion intensity and the $x$-axis the $m / z$ value. The score (Andromeda score) and the $m / z$ value of the parent ion are displayed above the spectrum. The upper panel displays the fragmentation spectrum whereas the lower panel indicates the respective $b$ - and $y$-ion series. lons that help to determine the phosphorylation site position are highlighted with a box. For spectra annotation, MaxQuant Viewer expert annotation was used. The annotated spectra were further modified with Illustrator (v5.1) for better legibility and the indication of ions of special interest. (A) The neutral loss b-ion b3* and the b3ion indicate the phosphorylation of one of the three $\mathrm{N}$-terminally located serines. The $\mathrm{y}$-ions $\mathrm{y} 21^{2+}$ and $\mathrm{y} 2 \mathrm{O}^{2+}$ localize the site of phosphorylation unambiguously to position S363. (B) The neutral loss b-ion b3* indicates the phosphorylation of one of

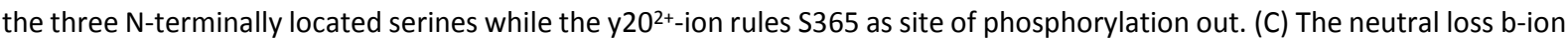
b3* indicates the phosphorylation of one of the three $\mathrm{N}$-terminally located serines.

\subsubsection{BRAF interactome}

BRAF involvement in antigen-dependent BCR signaling in DG75 was further investigated by the analysis of its interactome. BRAF is a serine-threonine kinase, namely a MAPKKK (or MAP3K) and therefore an upstream kinase in the classical MAP-kinase signaling cascade (reviewed in Chang and Karin, 2001 and Dhillon et al., 2007). As BRAF is not described to be mutated in Burkitt's lymphoma, for example the V600E point mutation leading to its constitutive activation, the kinase is amenable to activity regulation (Davies et al., 2002). As other MAP-kinases, BRAF activation depends amongst other processes on activating and inhibiting of phosphorylation sites and their dephosphorylation (reviewed in Chang and Karin, 2001 and Dhillon et al., 2007). BRAF is therefore expected to interact with its upstream interactors as well as with its downstream targets. The interactome analysis to identify putative BRAF binding partners was performed with a triple SILAC experimental design: lightand medium-labeled DG75 cells were left non-stimulated whereas the BCRs of heavy-labeled cells were stimulated for 5 min via their BCR (Figure 3.10 A). BRAF was purified with a BRAFspecific antibody from the medium and heavy cell lysates (Figure 3.10 A). As a control, the light-labeled lysates were incubated with the beads lacking the antibody. Immunopurified proteins were mixed in equimolar amounts, separated by SDS-PAGE, digested with trypsin and analyzed by LC-MS/MS (Figure 3.10 A). Of note, the DG75 cells used for the BRAF interactome analysis were affected to some extent by metabolic arginine-to-proline conversion as described in 3.1.12 and shown in Figure 3.13 E (Blagoev and Mann, 2006; Lössner et al., 2011; Ong et al., 2003; Van Hoof et al., 2007). However, the distribution of the normalized protein ratios was normally distributed and specific BRAF enrichment could be observed. Therefore results were used for analysis but might be considered carefully. 
Highly specific enrichment of BRAF by immunopurification compared to the control state is demonstrated by high medium-to-light $(\mathrm{M} / \mathrm{L})$ and heavy-to-light $(\mathrm{H} / \mathrm{L})$ quantitation ratios (right upper quadrant; Figure 3.10 B). As a proof-of-principle, the downstream MAP-kinase of BRAF, MAP2K1 (MEK1) was found to be enriched together with BRAF in both the nonsimulated (medium) and the BCR stimulated cells (heavy) (reviewed in Chang and Karin, 2001; Dhillon et al., 2007). The diagonal distribution of protein $H / L$ and $M / L$ SILAC ratios showed a Pearson's correlation efficient of 75.9\%, indicating correlation in the binding behaviour of BRAF interactors after 5 min of BCR stimulation compared to the non-stimulated state. The majority of proteins (grey squares) cluster around the intersection of the $y$ - and $x$-axis base lines and presumably present non-specific binders (light grey dashed lines, Figure $3.10 \mathrm{~B}$ ). 
A

No BCR stimulation No BCR stimulation 5' BCR stimulation

B

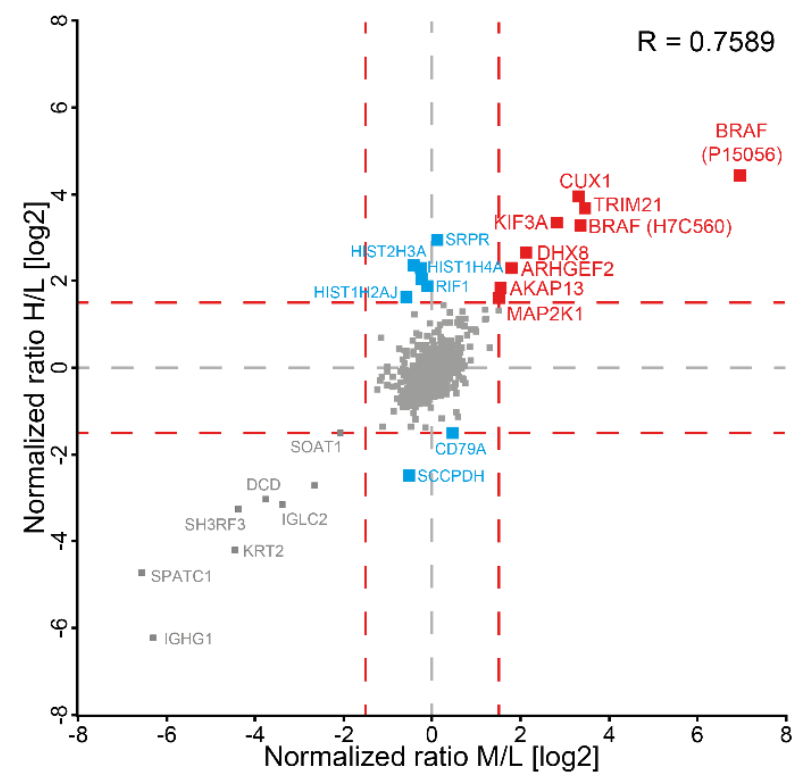

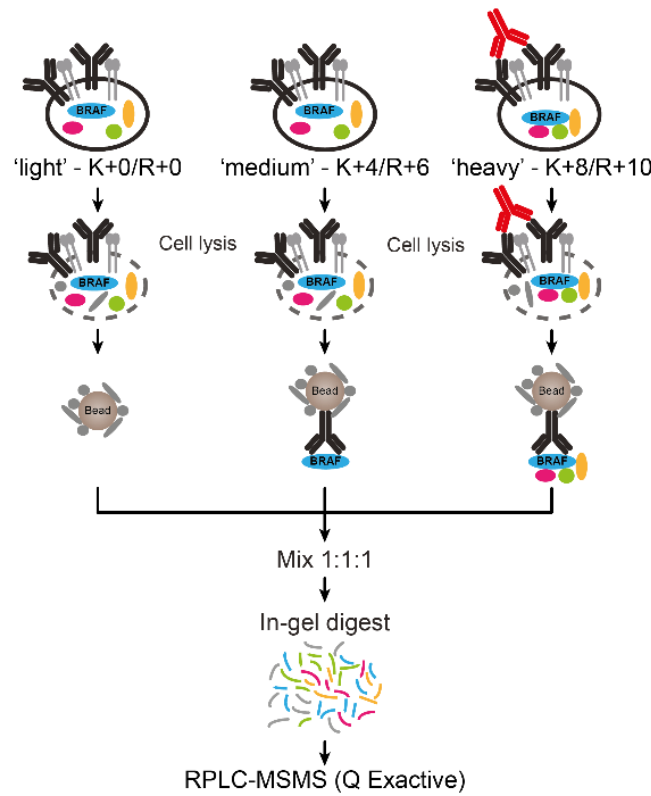

C

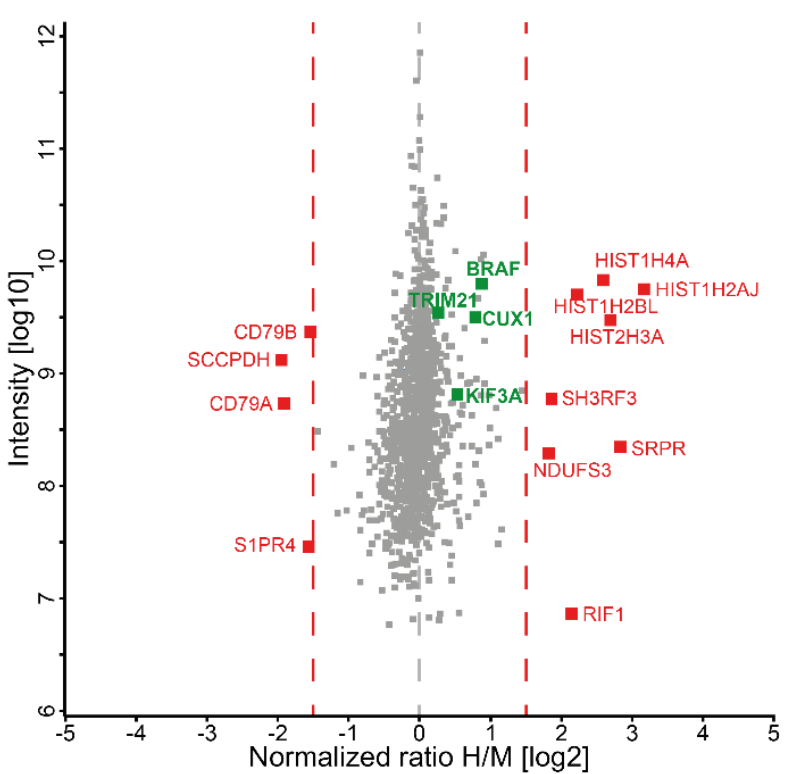

Figure 3.10: BRAF interactome analysis in DG75

(A) For the BRAF interactome analysis, light- and medium-labeled DG75 cells were left non-stimulated whereas the BCRs of heavy-labeled cells were stimulated for $5 \mathrm{~min}$. BRAF was affinity purified from the medium and heavy lysates with a BRAFspecific antibody. As a control, the light-labeled lysates were incubated with the beads lacking the antibody. Immunopurified proteins were mixed in equimolar amounts, separated by SDS-PAGE, digested with trypsin and analysed by LC-MS/MS. (B) The scatter plot shows the distribution of the normalized SILAC heavy-to-light $(\mathrm{H} / \mathrm{L}$ ) ratio of proteins ( $\mathrm{y}$-axis) reflecting $5 \mathrm{~min}$ of $B C R$ stimulation and the medium-to-light $(M / L)$ ratio ( $x$-axis; both $\log 2$ ) reflecting the non-stimulated ground state. Interaction partners with ratios $\leq-1.5$ and $\geq 1.5$ are highlighted in red and gene names are indicated, proteins below the threshold (red dashed lines) are marked in grey. Proteins with a H/L ratio above or below the threshold are highlighted in blue. The vertical and horizontal base lines are shown as light grey dashed line. The Pearson's correlation coefficient is displayed in the upper right corner of the scatter plot. BRAF is represented two times in the scatter plot due to two different sequence database entries (C) The scatter plot shows the distribution of the intensity ( $y$-axis; log10) against the normalized SILAC ratio $\mathrm{H} / \mathrm{M}$ (x-axis; log2). The H/M ratio reflects binding after $5 \mathrm{~min}$ of BCR stimulation compared to ground state. Proteins highlighted in green are the most abundant seen in (B). The threshold and color code settings are mentioned in (B). All detected proteins are listed in supplementary table S6. 
The proteins enriched together with BRAF in the ground and the BCR stimulated state in the upper right quadrant: CUX1, TRIM21, KIF3A, DHX8, ARHGEF2, AKAP13 and MAP2K1 could potentially be interaction partners of BRAF (highlighted in red, Figure $3.10 \mathrm{~B}$; supplementary table S6). Potential contaminant proteins were depleted in the medium- and heavy-labeled state i.e. enriched in the light-labeled control experiment and appeared in the left lower quadrant: IGHG1, SPATC1, KRT2, SH3RF3, DCD, IGLC2, CRYAA and SOAT1. Proteins specifically depleted (CD79A and SCCPDH) or enriched (SRPR, RIF1 and histone proteins) only after 5 min of BCR stimulation (heavy label) and not in the non-stimulated (medium label) state are highlighted in blue (Figure $3.10 \mathrm{~B}$; supplementary table S6). No protein was found to be specifically depleted in one state and enriched in the other state (right lower or the left upper quadrant). To further link the experimental BRAF interaction data with the BCR stimulation data in DG75, p-site and protein expression levels of potentially interesting interacting candidates were mapped. For three proteins with the highest SILAC ratios, CUX1, TRIM21 and KIF3A, no regulated $p$-site could be mapped from the phosphoproteomic dataset and no protein expression change could be detected over the time course of stimulation (supplementary table S6).

To thoroughly visualize BCR stimulation induced partners, the normalized heavy-to-medium (H/M) SILAC ratios which directly compare 5 min of BCR stimulation to the ground state were plotted against the $\log 10$ intensity (Figure $3.10 \mathrm{C}$ ). The majority of proteins did not show an altered binding behaviour as can be seen by the evenly distribution around the vertical zero line (light grey dashed line) and SILAC ratios between -1 and 1 on a log2 scale (grey squares). BRAF and its co-purified putative interaction partners (CUX1, TRIM21 and KIF3A) shown in Figure $3.10 \mathrm{~B}$ are highlighted in green (Figure 3.10 C). Potential BRAF interaction partners enriched prior to BCR stimulation can be found on the left side of the scatter plot: CD79A/B (signaling module of the BCR), SCCPDH, and S1PR4 whereas putative BRAF interactors after 5 min of BCR engagement can be found on the right side of the scatter plot: HIST1H2BL/AJ, HIST1H4A, HIST2H3A, SH3RF3, SRPR, NDUFS3, and RIF1.

\subsubsection{BRAF inhibition}

After the identification of the activation-induced BRAF interactome, BRAF downstream actions in antigen-dependent BCR signaling in DG75 were analysed. This was accomplished by BCR stimulation in combination with BRAF inhibition in DG75 cells followed by a global 
phosphoproteome analysis (Figure 3.11 A). As experimental design a triple SILAC labeling approach was used: light-labeled cells were left non-stimulated, the BCRs of medium- and heavy-labeled cells were both stimulated for $5 \mathrm{~min}$, respectively. In addition to BCR stimulation, the heavy-labeled cells were treated with the small-molecule BRAF inhibitor Raf265 (Figure 3.11 A) (Williams et al., 2015). Of note, the DG75 cells used for this experiment were also affected to some extent by arginine-to-proline conversion as described in 3.1.12 and shown in Figure 3.13 F (Blagoev and Mann, 2006; Lössner et al., 2011; Ong et al., 2003; Van Hoof et al., 2007). As normalized protein and p-site ratios were approximately normally distributed and centered around zero, results were used for analysis but might be considered carefully (Figure $3.11 \mathrm{~B}$ ). Moreover, $\mathrm{M} / \mathrm{L}$ ratios appeared to be more severely affected by this effect as can be seen by a stronger indirect correlation of the shift towards light and proline content compared to the heavy-to-light ratios (Figure 3.13 F, left panel: M/L, right panel: $\mathrm{H} / \mathrm{L}$ ).

A

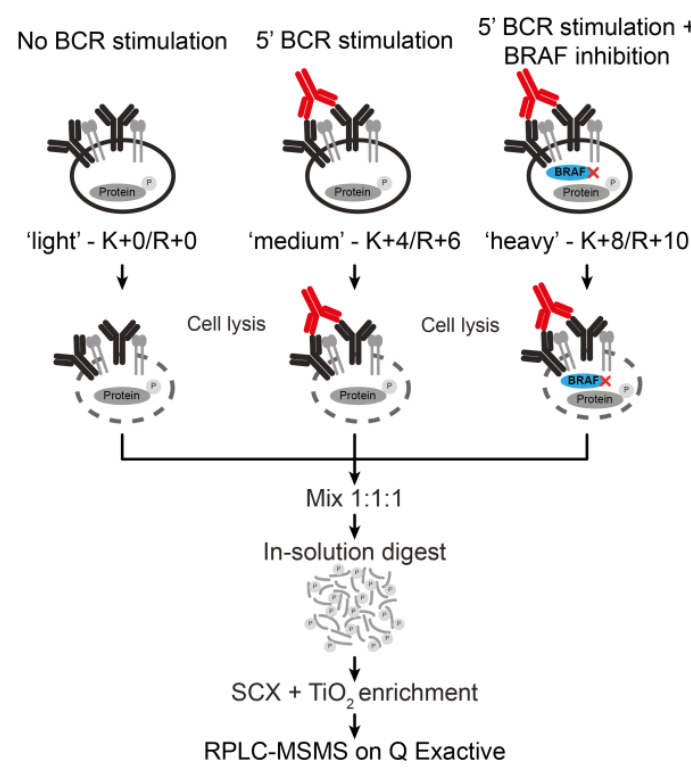

B

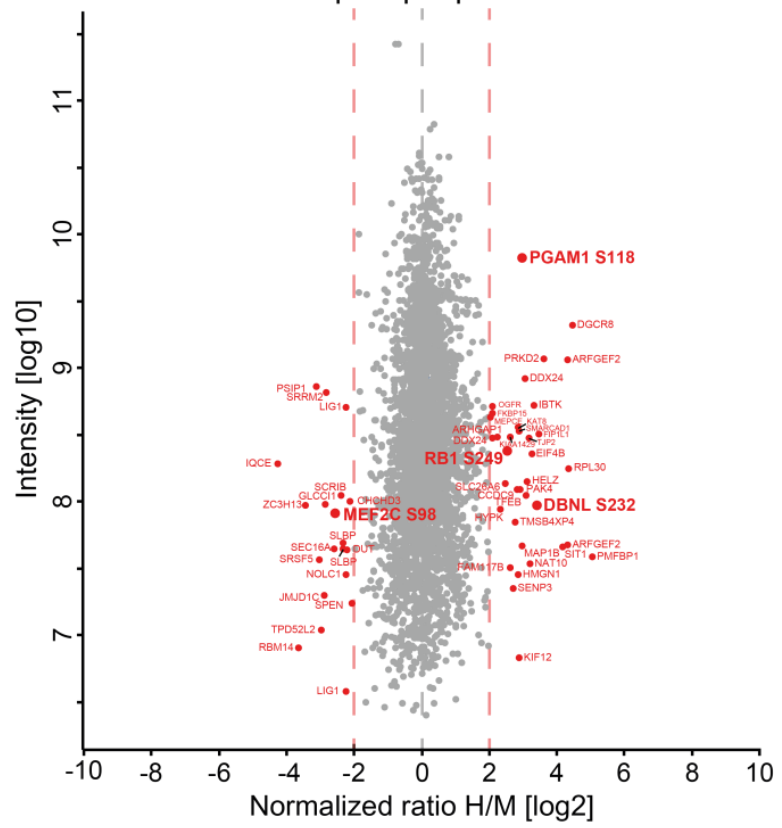

Figure 3.11: BCR signaling during BRAF inhibition

(A) Experimental design of the BRAF inhibition analysis in DG75 cells. Light-labeled DG75 cells were left non-stimulated whereas the BCRs of medium- and heavy-labeled cells were stimulated for $5 \mathrm{~min}$. Additionally, heavy-labeled cells were treated with the BRAF inhibitor Raf265 for 45 min before BCR stimulation. Cells were lysed; the different labeling states were mixed in equimolar amounts. Lysates were digested in-solution and enriched for phosphopeptides with the $\mathrm{SCX}+\mathrm{TiO}_{2}$-based enrichment and subjected to LC-MS/MS analysis. (B) The $y$-axis of the scatter plot shows the intensity values $(\log 10)$ and the $x$-axis the normalized heavy-to-medium (H/M) SILAC ratios ( $\log 2)$ of individual class I phosphorylation sites. P-sites with a SILAC ratio between -2 and 2 on a log2 scale are colored in grey, sites with lower or higher ratios are colored in red and gene names are indicated. Phosphorylation sites specifically mentioned in the text are highlighted in bold and sites are indicated. All identified $\mathrm{p}$-sites are listed in supplementary table S7. 
From the global BRAF inhibition phosphorylation site analysis, 3845 class I p-sites could be quantified upon 5 min BCR stimulated cells with pharmacological inhibited BRAF (heavy label) and 5 min BCR stimulated cells (medium label; Figure $3.11 \mathrm{~A}$; supplementary table S7). The identified BRAF p-sites S151, S446 and S729 did not show phosphorylation changes in the different experimental conditions (supplementary table S7). 57 p-sites on 52 effector proteins were found with normalized $\mathrm{H} / \mathrm{M}$ ratios $\leq-2$ or $\geq 2$ on a $\log 2$ scale and were therefore considered to be regulated after BRAF inhibition. The effector proteins, MEF2C and DBNL were previously linked to BCR signaling (supplementary table S7) (Le Bras et al., 2004; Muzio et al., 2007; Swanson et al., 1998). The transcription activator MEF2C was found dephosphorylated at S98. No particular p-site annotation for this $p$-site is available, however the protein is associated with BCR signaling and plays a role in BCR survival and proliferation (Swanson et al., 1998). The second BCR effector protein, DBNL (HIP-55) showed an upregulated p-site at position S232 during Raf265 treatment. As for MEF2C, no particular functional information for the p-site S232 is available. However, DBNL is known to be involved in endocytosis and actin cytoskeleton processes in T-cell receptor signaling (Le Bras et al., 2004). Furthermore, two regulated phosphorylated $p$-sites were annotated according to PSP as regulatory sites: PGAM1 S118 and RB1 S249. PGAM1 S118 is an inhibitory site which is involved in metabolic processes to drive cancer (Hitosugi et al., 2012; Shalom-Barak and Knaus, 2002). The phosphorylation site S249 of the tumor suppressor RB1 is involved in cell cycle regulation function and was associated with breast cancer (Caldon et al., 2007). None of the two sites could be additionally detected in the BCR stimulation time course of DG75 cells (supplementary table S7). In summary, the serine-threonine kinase BRAF appeared to be an interesting candidate for further large-scale analyses. The BRAF interactome and the altered $p$-sites identified from the global phosphoproteomic analysis during inhibitor-induced BRAF ablation provided interesting insights in BRAF involvement in BCR signaling. However, the overall outcome is difficult to interpret and requires more replicates, methodical refinement and further functional validation.

\subsubsection{Acetylation and ubiquitination}

Besides phosphorylation, other PTMs are known to also play a major role in BCR signaling as described previously in section 1.3.8 (reviewed in Kurosaki et al., 2009). To complement the large-scale phosphorylation study, lysine ubiquitination and acetylation was additionally 
analyzed in DG75 in a double-label SILAC approach, respectively. Light-labeled cells were not stimulated whereas the BCRs of heavy-labeled cells were stimulated for 2 min (Figure $3.12 \mathrm{~A}$ ). Peptides were immunopurified analogous to the phosphotyrosine enrichment with commercially available antibodies as previously described 2.2.2.2.2 directed against the ubiquitin di-glycine remnant at lysine residues or acetylated lysine residues. The DG75 samples used for this analysis were affected by metabolic arginine-to-proline conversion as described in 3.1.12 (data not shown) (Blagoev and Mann, 2006; Lössner et al., 2011; Ong et al., 2003; Van Hoof et al., 2007). Nonetheless, the results were used for analysis as the experiments were primarily conducted as a feasibility and screening study but have to be considered carefully.

Figure $3.12 \mathrm{~B}$ shows the normalized SILAC ratios of 372 quantified class I ubiquitination sites derived from 224 proteins. 32 sites derived from 21 proteins were considered as regulated with SILAC ratios <-2 or $>2$ (Figure $3.12 \mathrm{~B}$, highlighted red; Figure $3.12 \mathrm{D}$, upper panel). Of particular interest was the increase in ubiquitination of CD79B K219 after 2 min of BCR stimulation (Figure 3.12 B, highlighted bold). The site lies on the intracellular domain of CD79B, C-terminal of the ITAM-motif and is not yet functionally described (supplementary table S8). From the acetylation study, 148 class I sites on 93 proteins could be quantified (Figure $3.12 \mathrm{C}$ ). Five acetylated lysine residues on distinct different proteins were quantified with a SILAC ratio $<-2$ or $>2$ (Figure $3.12 \mathrm{D}$, upper panel). 
A

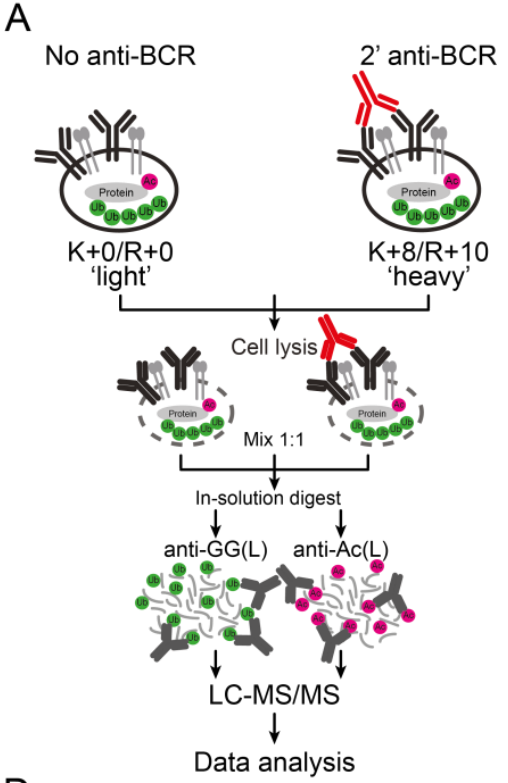

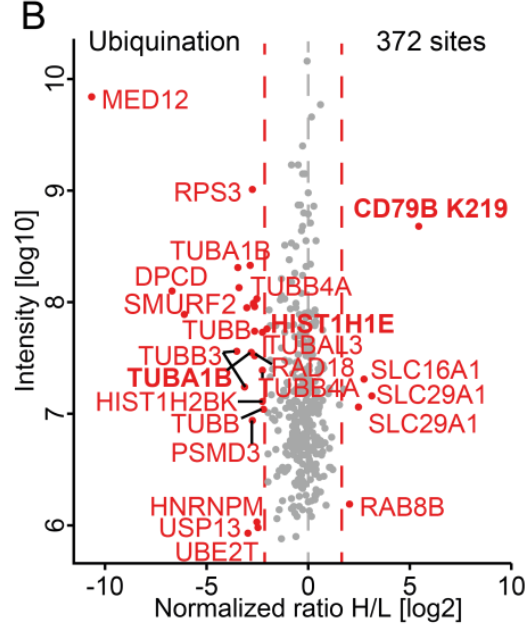
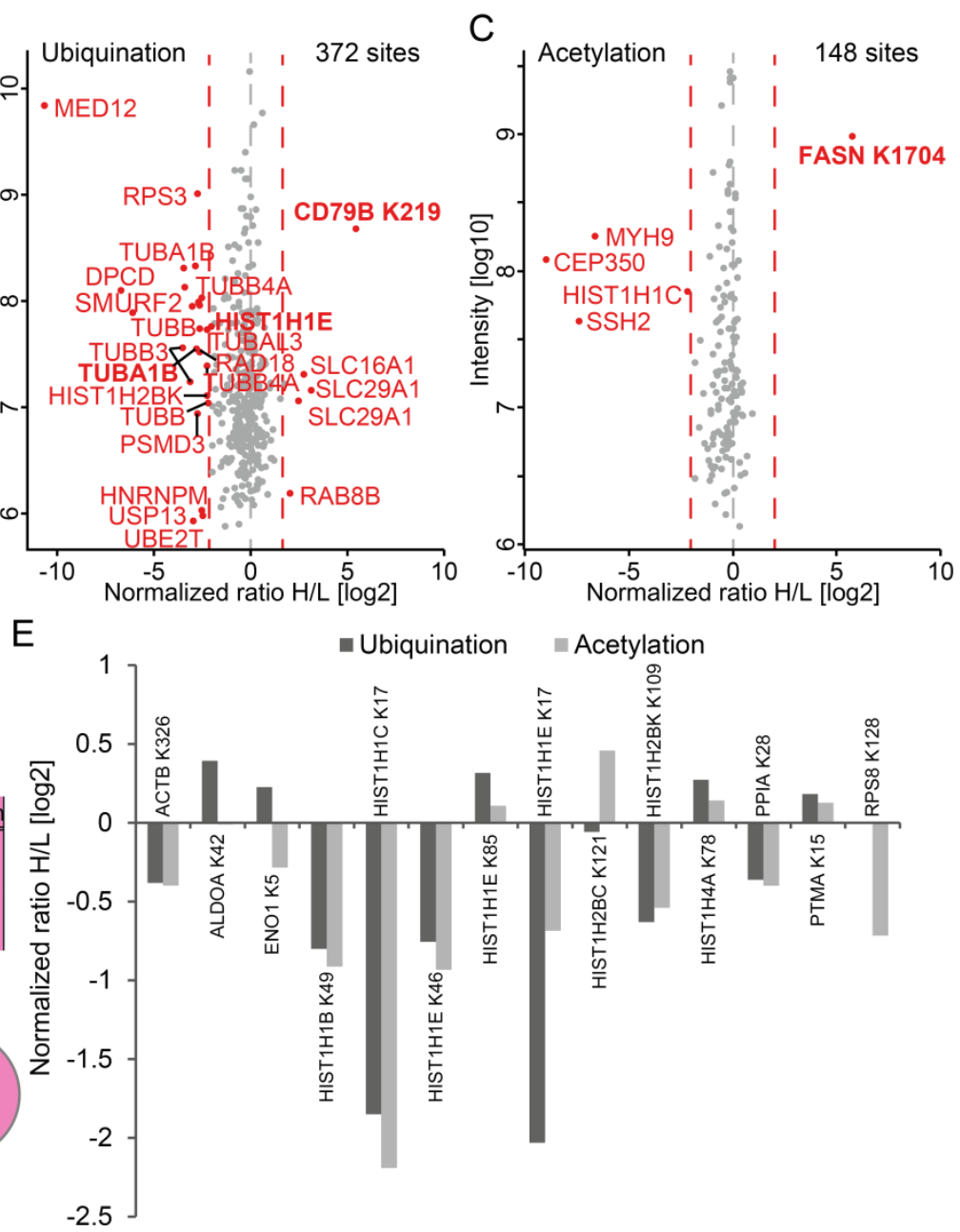

Figure 3.12: Ubiquitination and acetylation in BCR signaling

(A) Experimental design of the acetylation and ubiquitination analysis in DG75 cells. Light-labeled DG75 cells were left nonstimulated whereas the BCRs of heavy-labeled cells were stimulated for $2 \mathrm{~min}$. Cells were lysed, the two labeling states were mixed in equimolar amounts. Proteins were digested in-solution, peptides were immunopurified with modification specific antibodies (GG(L): ubiquitin remnant motif, $A C(L)$ : acetyl-lysine; green=ubiquitination, pink=acetylation) and subjected to $L C$ MS/MS analysis. (B) and (C) Intensities ( $\log 10)$ of quantified class I ubiquitination and acetylation sites are plotted against the normalized heavy-to-light (H/L) SILAC ratios ( $\log 2)$. Modified sites with a ratio between -2 and 2 are colored as grey dots, sites with ratios lower or higher are highlighted as red dots and gene names are indicated. The dashed grey line represents the vertical zero line, the red dashed lines the regulation threshold. (D) Table listing the numbers (\#) of quantified class I sites, number of effector proteins, regulated sites and regulated effector proteins (upper panel). Ubiquitination is color-coded in green, acetylation in pink. The right Venn diagram shows the overlap of regulated effector proteins identified from the ubiquitination, acetylation and phosphorylation site analyses. The left Venn diagram displays the overlap of lysine sites being ubiquitinated and acetylated (both lower panel). (E) Comparison of the normalized SILAC H/L ratios ( $y$-axis) of the 14 lysine residues (lower left Venn diagram in D) that are ubiquitinated (dark grey bars) as well as acetylated (light grey bars). All quantified sites are listed in supplementary table $\mathrm{S} 8$.

When screening for positive PTM cross talk between ubiquitinated, acetylated and phosphorylated sites, no overlap in regulated effector proteins could be identified. However, a few ubiquitinated or acetylated proteins were found in common with phosphorylation, respectively (Figure 3.12 D, left lower panel). Fatty acid synthase (FASN) was the only protein identified with a regulated acetylation and phosphorylation site. FASN K1704 was acetylated 
after 2 min of BCR stimulation while T2204 became phosphorylated after 10 min of BCR stimulation (supplementary table S8). HIST1H1E, TUBA1B and TUBB were identified with regulated ubiquitination and phosphorylation sites. HIST1H1E K17 became deubiquitinated after 2 min while T18 became dephosphorylated after 5 min of BCR stimulation (supplementary table S8). Multiple TUBA1B phosphotyrosine sites (Y103, Y224, Y272, Y312, Y357 and Y399) were identified as upregulated whereas ubiquitination from all lysine sites (K60, K96, K304, K326, K336 and K370) was removed after BCR engagement (supplementary table S8). TUBB became phosphorylated at Y32 while K40, K324, K336 became deubiquitinated (supplementary table S8). To identify potential negative cross talk, both acetylation and ubiquitination site datasets were compared. 14 modified sites on twelve unique proteins e.g. ACTB, ENO1, PTMA and different histone proteins, were found to be modified by ubiquitination as well as acetylation (Figure 3.12 D, right lower panel and Figure 3.12 E). The bar diagram in Figure 3.12 E shows the normalized SILAC ratios of the 14 lysine class I sites that were found to be modified with acetylation and ubiquitination (Figure 3.12 D, left lower panel). Two common sites exceeded the regulation threshold with at least one type of modification (HIST1H1C K17 and HIST1H1E K17) whereas all other common modified lysine sites were considered as non-regulated. Eight of the common sites were responding accordingly to BCR stimulation. Five sites (ACTB K326, HIST1H1B K49, HIST1H1E K46, HIST1H2BK K109 and PPIA K28) were found to be deacetylated and -ubiquitinated. In contrast, HIST1H1E K85, HIST1H4A K78, and PTMA K15 became acetylated and ubiquitinated, respectively after BCR stimulation. ALDOA K42 and RPS8 K128 showed a change for only one modification where as ENO1 K5 and HIST1H2BC K121 were regulated in opposite directions, respectively (supplementary table S8). Overall, the general yield in acetylated and ubiquitinated sites was low, therefore less sites potentially involved in PTM cross talk were identified. Experiments have to be refined as will be discussed later in chapter 4 .

\subsubsection{Arginine-to-proline conversion}

Metabolic labeling strategies such as SILAC are one of the most robust quantitation methods available for mass spectrometry (Ong et al., 2002). As mentioned earlier in 1.3.6.1, an advantage is the early sample mixing and therefore parallel sample handling which makes the method robust against sample loss (Ong et al., 2002). However, its robustness is compromised by the metabolic conversion of arginine to proline, Arg+10 to Pro+6 and Arg+6 to Pro+5, 
respectively (Blagoev and Mann, 2006; Ong et al., 2003; Van Hoof et al., 2007). The conversion affects correct quantitation, as it lowers the intensity of the heavy arginine- and lysine-labeled SILAC peptides and introduces heavy satellite peaks derived from peptides containing additional heavy proline which are not taken into account for quantitation (Lössner et al., 2011). Strategies to avoid conversion are the reduction of the arginine concentration, the addition of unlabeled proline or the use of quantitation strategies to correct for the conversion (Bendall et al., 2008; Blagoev and Mann, 2006). A low arginine concentration is not well tolerated by every cell line and in silico correction is feasible if the sample complexity is low and samples are duplex labeled only i.e. light and heavy (Bendall et al., 2008; Park et al., 2009). For highly complex samples with triplex labeling and phosphorylation as further variable parameter, correction strategies are not feasible anymore. Unfortunately, arginine-to-proline conversion appeared in a few samples (BRAF interactome, BRAF inhibition, and the antigendependent BCR acetylation and ubiquitination signaling analyses in DG75, respectively) used for the investigation of Burkitt's lymphoma. The effect became obvious by global shifts of the SILAC ratios from the protein or modified peptide population towards light (data not shown). Retrospective, manual spectra investigation revealed complex SILAC patterns with satellite peaks with an additional mass of 5 or $6 \mathrm{Da}$ in proline containing peptides (an example is given in Figure $3.13 \mathrm{~A}$ ). Those satellite peaks were absent in peptides lacking proline (Figure $3.13 \mathrm{~B}$ ). In order to get an overview about the magnitude of conversion, the non-normalized SILAC peptide ratios of all experiments analyzed were plotted against the number of prolines contained in the peptide sequence and color-coded according to the density. Indirect correlation of the peptide SILAC ratio shifts with the peptide proline content indicates conversion (Figure 3.13 C-F). The BCR stimulation time course of DG75 cells used for the global phosphoproteome and the pYome analysis showed no or at least only minor proline conversion (Figure 3.13 C and D; Results described in section 3.1.3). The BRAF interactome experiments in DG75 were slightly affected (Figure 3.13 E; Results described in section 3.1.9). In contrast to that, DG75 cells treated with a BRAF inhibitor used for the global phosphoproteome analysis were affected more severely by arginine-to-proline conversion (Figure 3.13 F; Results described in section 3.1.10). The medium-labeled cells appeared to be affected to a higher extent by conversion as can be deduced from the greater inverse correlation compared to the heavy-labeled cells (Figure $3.13 \mathrm{~F}$, left and right panel, 
respectively). However, the experiments were taken into account for data analysis but need to be considered more cautiously.
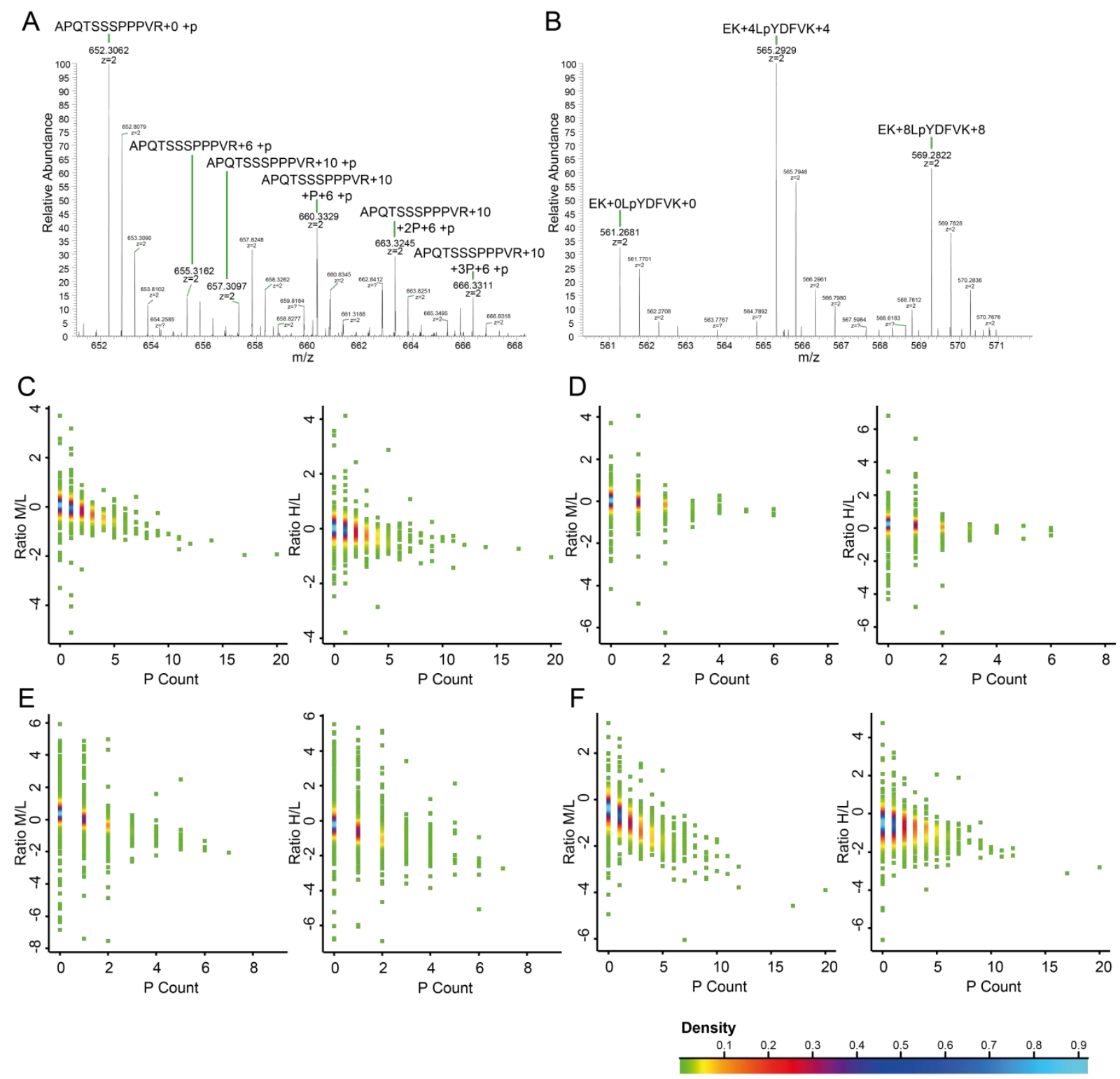

Figure 3.13: Arginine-to-proline conversion

(A) Parent ion spectrum of a doubly charged $(z=2)$, tryptic peptide APQTSSSPPPVR derived from the protein SRRM1, harboring one phosphorylation site on the threonine or serine residues (general indicated as $+p$ ). The peptide is derived from $B C R$ stimulated Daudi BL cells with triple SILAC labeling $(R+0, R+6$ and $R+10$; indicated in the MS spectrum) enriched for a global phosphoproteomic analysis. Medium $(R+6)$ and heavy arginine $(R+10)$ are converted in medium $(P+5)$ and heavy proline $(P+6)$, respectively. For simplicity reasons, only the peaks corresponding to up to three converted heavy prolines are highlighted in the MS spectrum $(P+6,2 P+6$ and $3 P+6)$. The $y$-axis displays the relative intensity of the peaks, the $x$-axis the $m / z$ values of the ions. (B) Parent ion spectrum of the doubly charged $(z=2)$, tryptic peptide EKLYDFVK derived from the protein INPP5D. The peptide lacks proline and contains two labeled lysines $(2 x \mathrm{~K}+0,2 x \mathrm{~K}+4$ and $2 x \mathrm{~K}+8)$ due to a missed cleavage site and additionally, the tyrosine residue at position four is phosphorylated (indicated as $p$ in the amino acid sequence). The sample is derived from the same experimental sample mentioned above. The peaks corresponding to the three SILAC states are highlighted in the MS spectrum. Axis legend can be found in (A). (C) - (I) Density plots of different experimental setups analyzed for arginine-to-proline conversion. The $x$-axis shows the number of prolines in the peptide sequence ( $P$ count), the $y$-axis the non-normalized peptide SILAC ratios while the dots are color-coded by density (see legend). (C) One of four biological replicates of a DG75 BCR stimulation time course with global phosphoproteome enrichment (D) One of two 
biological replicates of a DG75 BCR stimulation time course with pYome enrichment (E) DG75 BRAF interactome analysis (F) Global phosphoproteome enriched DG75 cells after BRAF inhibition.

\subsubsection{Antigen-dependent BCR signaling in three distinct Burkitt's lymphoma cell lines}

The antigen-dependent signaling dynamics in DG75 were additionally compared to two other Burkitt's lymphoma cell lines on the pYome level: the EBV-positive, endemic Burkitt's lymphoma cell line Daudi and a primary cell line (FFM_BL1 or BL1), derived from a patient suffering from BL. Further details about the mutational background for the cell lines are described in Table 9. Daudi cells were triplex SILAC labeled: light-labeled cells were left nonstimulated, the BCRs of medium- and heavy-labeled cells were stimulated for 2 min and 10 min, respectively. The patient derived Burkitt's lymphoma cells could only be cultivated for a short time under cell culture conditions, therefore metabolic labeling was not amendable and cell numbers were sufficient for one biological replicate only. As alternative quantitation strategy, a label-free quantitation approach was chosen. BL1 cells were also stimulated for 2 and $10 \mathrm{~min}$ via their BCRs, respectively. To make the different datasets more easily comparable, raw files from the DG75 and Daudi pYome SILAC experiments were jointly submitted to MaxQuant processing and the results matched to the independently processed label-free BL1 dataset as described in 2.2.5.3.

577 p-sites could be quantified after antibody-based phosphotyrosine enrichment in DG75 cells, in at least two time points of BCR stimulation (Figure 3.14 A). The Daudi pYome analysis lead to the quantitation of 304 p-sites and BL1 cells lead to the quantitation of $325 p$-sites at both stimulation time points, respectively (Figure 3.14 A). 165 p-sites were found commonly in all three cell lines (Figure $3.14 \mathrm{~A}$, supplementary table S9). As a first step, the 165 common p-sites were subjected to principal component analysis (PCA) to visualize differences between the different BL cell lines and time-dependent BCR stimulation (Figure 3.14 B). Component 2 and 3 separated the EBV-positive Daudi cell from the other cell lines (Figure 3.14 B). The 'early' (including 2 and $5 \mathrm{~min}$ ) and the 'late' time points (including 10 and $20 \mathrm{~min}$ ) after BCR stimulation of both the sporadic BL cell lines DG75 and BL1 clustered together, rather than clustering according to the cell line (Figure 3.14 B). To extract accordingly upregulated p-sites in all three cell lines, p-sites with SILAC ratios greater than 1 on a log2 scale in at least one BCR stimulation time point were filtered (supplementary table S9). This lead unsurprisingly to the identification of 24 p-sites on 19 mostly upstream BCR signaling effectors such as CBL, CD19, 
INPP5D, LAT2, MAPK1, MAPK3, NCK1, PIK3AP1, PLCG2, and SYK (supplementary table S9). 165 common p-sites were further subjected to Z-scoring and hierarchical cluster analysis to highlight quantitative differences between p-sites in different cell lines (Figure 3.14 C). The population of $p$-sites fell into three distinguishable clusters of which the largest comprised of 104 p-sites (light blue row tree, Figure 3.14 C). From this largest cluster a smaller sub-cluster was extracted that contained differential BL cell line-dependent p-sites (dark blue row tree, Figure $3.15 \mathrm{C})$. This cluster consisted of $65 \mathrm{p}$-sites derived from 51 effector proteins (supplementary table S9). 


\section{Results}

A

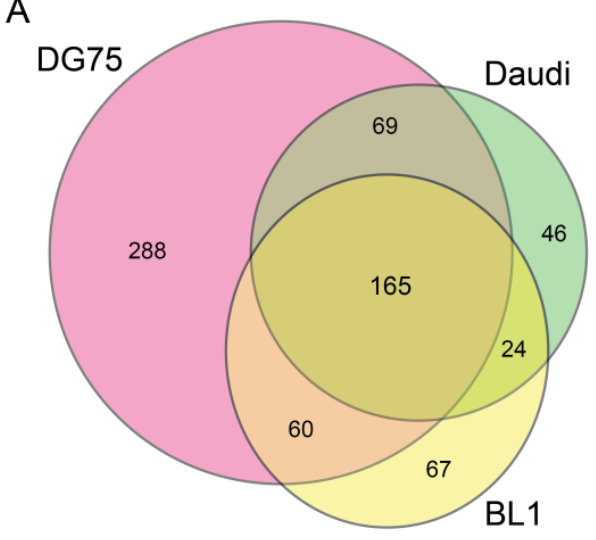

C

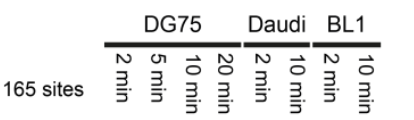

$\mid$
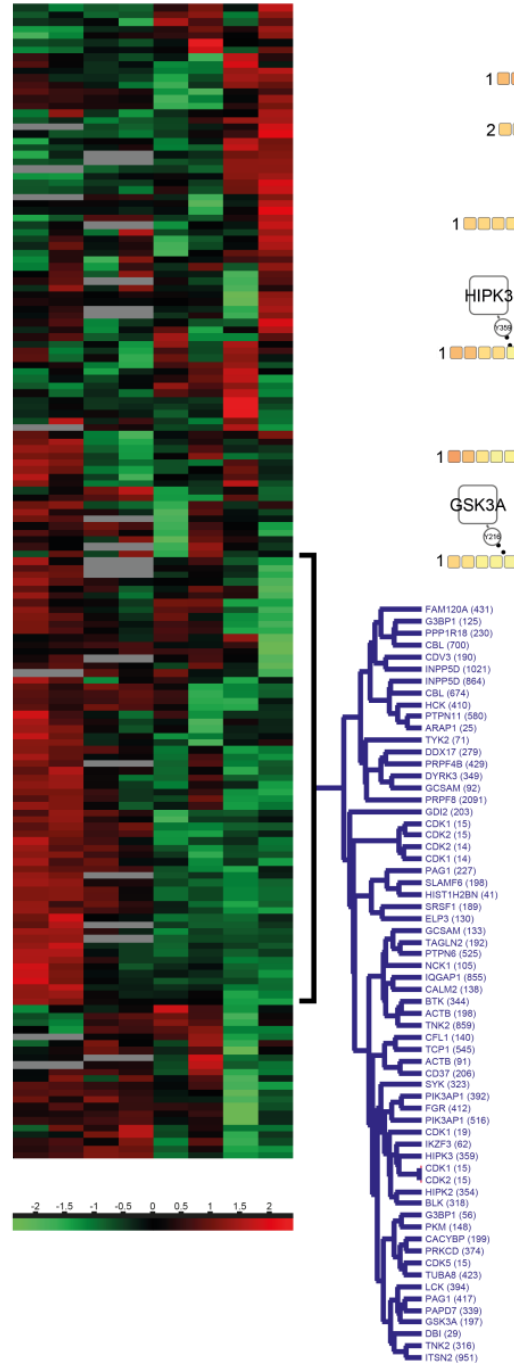

B
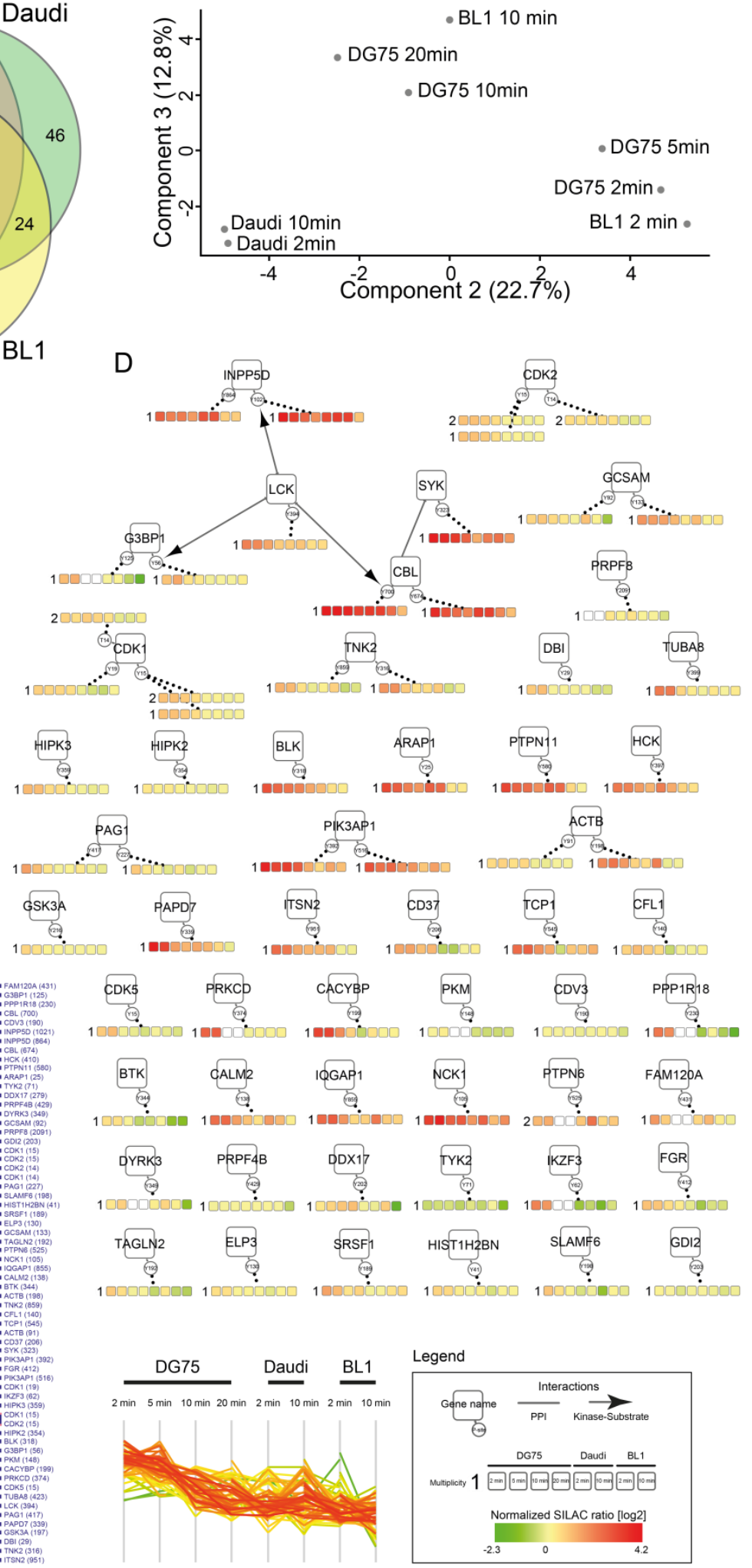

Figure 3.14: Antigen-dependent BCR signaling in three distinct BL cell lines

(A) The Venn diagram shows the number of individual and commonly quantified p-sites (not proportionally) in three distinct Burkitt's lymphoma cell lines, DG75, Daudi and FFM_BL1 (or BL1) after B-cell receptor stimulation derived from the respective pYome analyses (B) B-cell line and BCR engagement-dependent PCA analysis (C) Heat map of 165 common p-sites quantified in DG75 (in at least two of four time points), Daudi and FFM_BL1 primary cells. Normalized SILAC ratios (log2) were Z-scored and subjected to hierarchical cluster analysis. Z-scored p-site SILAC ratios (row) of all experimental conditions (column) are colored according to color scale at the bottom. The dataset was split in three distinct clusters and additionally, the most 
differential sites were manually extracted. The extracted row tree, gene names, the position of the phosphorylated amino acid (in brackets) and the phosphorylation profile of the cluster are shown right to the heat map (D) PhosphoPath network analysis of the most differential sites (see C) (Raaijmakers et al., 2015). Proteins were subjected to protein-protein and additionally $p$-sites to kinase-substrate interaction analysis (according to BioGRID, STRING and PSP, respectively). Proteins are represented by a rounded rectangle, $p$-sites by ellipses. The quantitative SILAC ratio $(\log 2)$ information across all time points and conditions is plotted below each $p$-site. Each rectangle represents a different cell line and time point. Quantitative values are color-coded (see legend), sites not quantified are coloured in white. Gene names and the position and amino acid of modification are indicated within the respective nodes. PPI interactions are represented by a line, kinase-substrate interactions by an arrow. PPI: Protein-protein interaction. P-site positions indicated in the figure dependent on the respective protein isoform and are listed together with all p-sites and quantitation values in supplementary table S9.

Protein and quantitative p-site information was subjected to PPI and kinase-substrate interaction analysis via PhosphoPath (Figure 3.14 D) (Raaijmakers et al., 2015). Solely five effectors, could be integrated in a functional interaction network (INPP5D, G3BP1, LCK, CBL and SYK) whereas the majority of proteins were not annotated as interacting (Figure 3.14 C), INPP5D, LCK, CBL and SYK are well known effectors of BCR signaling (reviewed in Kurosaki et al., 2009, Rickert, 2013 and Young and Staudt, 2013). G3BP1 (Ras GTPase-activating proteinbinding protein 1) is a phosphorylation-dependent endoribonuclease not yet directly associated with BCR signaling (Tourrière et al., 2001). Two phosphorylation sites, G3BP1 Y56 and $\mathrm{Y} 125$, localized within the nuclear transport factor 2 domain were commonly quantified in all cell lines. G3BP1 Y56 became phosphorylated after 2 and 5 min BCR stimulation in DG75 cells (1- and 1.4-fold, respectively) whereas in Daudi no, and in BL1 only a slight regulation behavior (0.3-fold increase after 10 min of BCR stimulation) was detected (Figure $3.14 \mathrm{D}$; supplementary table S9). G3BP1 Y125 became phosphorylated in DG75 after 2 and 5 min of BCR engagement (1- and 1.3-fold, respectively) while in Daudi no regulation behavior could be observed. In BL1, the G3BP1 Y125 was detected as dephosphorylated after 2 and 10 min of BCR stimulation (0.6-fold and 3.1-fold decrease after $2 \mathrm{~min}$ and $10 \mathrm{~min}$, respectively; Figure 3.14 D). Interestingly, G3PB1 has been associated with MYC RNA cleavage thereby promoting its decay (Tourrière et al., 2001). In the following, a few p-sites of not interconnected proteins shown in Figure $3.14 \mathrm{D}$ will be briefly discussed (Figure 3.14; supplementary table S9). ITSN2 (Intersectin-2) is a multidomain ( $\mathrm{SH} 3, \mathrm{PH}, \mathrm{C2}, \mathrm{DH}, \mathrm{EH}, \mathrm{EF}-$ hand) adapter protein, involved in clathrin-mediated endocytosis (Pucharcos et al., 2000). The phosphorylation site Y951 (corresponding to Y968 in isoform 1) was upregulated in response to BCR stimulation in DG75 (2.4- and 2.2-fold after 2 and $5 \mathrm{~min}$, respectively) and in Daudi cells (1.1- and 1.2-fold after 2 and 10 min, respectively) whereas no regulation of ITSN2 Y951 was detected in BL1 cells (Figure 3.14 D). The site lies in the linker region between two SH3 domains and was shown to be dependent on the activation of another receptor, namely the EGFR (Huang et al., 2007). Additionally, the site was identified in many large-scale analyses across different diseases 
models and cell lines (according to UniProt and PSP database information) but was not further functionally characterized. Another p-site that showed substantial differences between the three model cell lines was CD37 Y206. CD37 (Leukocyte antigen CD37) is expressed on mature human B cells as well as on human B-cell leukemia and lymphoma (Norton and Isaacson, 1987; Smith et al., 1989). It belongs to the tetraspanin family of membrane proteins characterized by four transmembrane domains. The protein family is functionally associated with antigen presentation, T-cell and B-cell interaction and adhesion (Knobeloch et al., 2000; SchwartzAlbiez et al., 1988; Tarrant et al., 2003). In DG75 cells, CD37 Y206 (in isoform 2, corresponding to Y274 in isoform 1) was constantly upregulated during the BCR stimulation time course (supplementary table S9). In contrast, CD37 Y206 was identified as downregulated in Daudi cells (0.7- and 0.8-fold, respectively) while in BL1 cells the p-site was not regulated (supplementary table S9). IKZF3 (Zinc finger protein Aiolos) is a transcription factor that regulates B-cell differentiation, proliferation and maturation (Wang et al., 1998). It regulates the expression of the anti-apoptotic factor $\mathrm{Bcl} 2$ and associates with anti-apoptotic $\mathrm{BCl}-\mathrm{X}_{\mathrm{L}}$, thereby controlling apoptosis (Rebollo et al., 2001; Romero et al., 1999). Phosphorylation of IKZF3 Y286 is associated with nuclear localization whereas the functional association of the other phosphorylation sites is characterized (Antica et al., 2007). IKZF3 Y62 (corresponding to Y96 in isoform 1) became upregulated in DG75 after 2 and 5 min of BCR stimulation (2- and 1.7-fold, respectively). In contrast, IKZF3 Y62 was dephosphorylated at both times points after BCR engagement in Daudi as well as in BL1 cells (1- and 0.6-fold in Daudi and 1.6- and 0.3-fold in BL1 at 2 and 10 min, respectively; supplementary table S9).

\subsubsection{Tonic BCR signaling}

Normal B cells as well as Burkitt's lymphoma cells critically depend on expression of a functional B-cell receptor (Kraus et al., 2004). BCR engagement by anti-lgM antibodies mimics a stimulus similar to antigen binding. Antigen-dependent signaling was intensively analyzed in this work under various experimental conditions (section 3.1.1 to 3.1.11). However, Burkitt's lymphoma survival and proliferation is thought to be mainly driven by tonic BCR signaling (Lam et al., 1997; Schmitz et al., 2012). In order to also investigate the much weaker signals derived from antigen-independent BCR signaling, two tonic signaling conditions were experimentally designed in DG75 as described in 2.2.1.3.2. First, cells were genetically modified with an inducible CD79A knockdown construct. CD79A is the signaling module of the BCR harbouring 
an ITAM-motif with two phosphorylatable tyrosine residues (Reth, 1989). Former studies showed that the knockdown of the BCR signaling module decreases the expression of the whole BCR complex on the cell surface (Lam et al., 1997). Therefore, BCR signals are shut down and remaining signaling emanates from the multitude of all other cell surface receptors. Diminished surface BCR expression could be observed in DG75 after CD79A knockdown (data not shown). Second, SYK, a central tyrosine kinase in BCR signaling was pharmacologically halted with the small-molecule inhibitor PRT062607 (Spurgeon et al., 2012). Both experimental states were compared to the BCR stimulated (wild-type BCR expressing) cells on the pYome level. These approaches were sought to determine the signals derived from tonic BCR signaling. The analyses of tonic signaling were performed as a double SILAC experiment in DG75: in light-labeled cells, the BCR was knocked down via a CD79A-specific, tetracyclininducible shRNA while heavy wild-type BCR expressing cells were left non-stimulated. In the second tonic signaling condition, SYK was inhibited in the heavy state while the light-labeled cells were left untreated. The decreased expression of surface BCR after the knockdown was verified by western blot analysis (data not shown). The diminished expression of CD79A after knockdown was validated by protein expression analysis while SYK expression did not change after inhibition (Figure 3.15 A). Antibody-based enrichment of phosphorylated tyrosine peptides identified 325 class I p-sites after BCR knockdown (BCR kd) and 489 class I p-sites after SYK inhibition (SYK-). 253 class I p-sites (239 pY p-sites) were commonly found in both conditions (Figure 3.15 B; supplementary table S10). Scatter plot analysis revealed hardly any correlation between the quantified p-sites upon BCR knockdown and SYK inhibition (Figure $3.15 \mathrm{C})$. Overall, among the proteins with altered $\mathrm{p}$-sites in at least one tonic signaling condition only a few belong to the class of standard BCR signaling proteins such as PLCG2, LYN, SYK, MAPK1/3 and CD79B (Figure 3.15 C). CD79B T195 (in isoform 1, corresponding to T91 in isoform 2; Figure $3.16 \mathrm{C}$; supplementary table S10) and CD79A Y210 (Figure $3.16 \mathrm{D}$; supplementary table S10) were found dephosphorylation after BCR knockdown. This finding can be attributed to a general low expression of the BCR complex after knockdown. During Syk inhibition CD79B T195 phosphorylation was not changed, whereas CD79A Y210 could not be identified (Figure 3.15 C). LYN Y194 which is located within the SH2 domain was found phosphorylation after CD79A ablation while no change could be detected after SYK inhibition. The activity determining MAP-kinase TXY motif was found to be dephosphorylated at Y178 (in MAPK1) and Y204 (in MAPK3) in both tonic signaling conditions, respectively. Furthermore, 
the Src-target site PRKCD Y313 located in a hinge region and being associated with regulating serine-threonine kinase activity was found to be dephosphorylated in both tonic signaling models (Figure 3.15 C; supplementary table S10) (Hall et al., 2007).

A

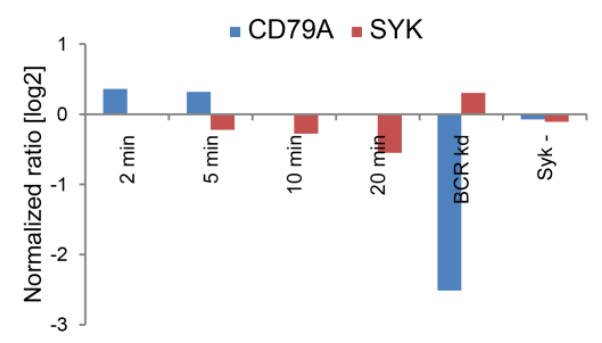

B

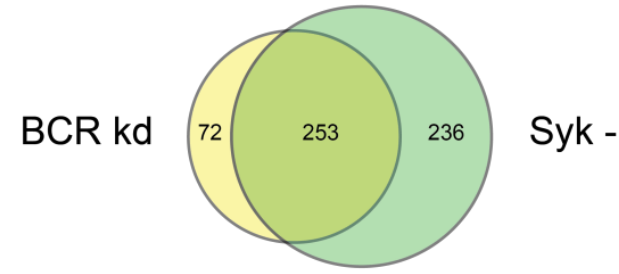

D

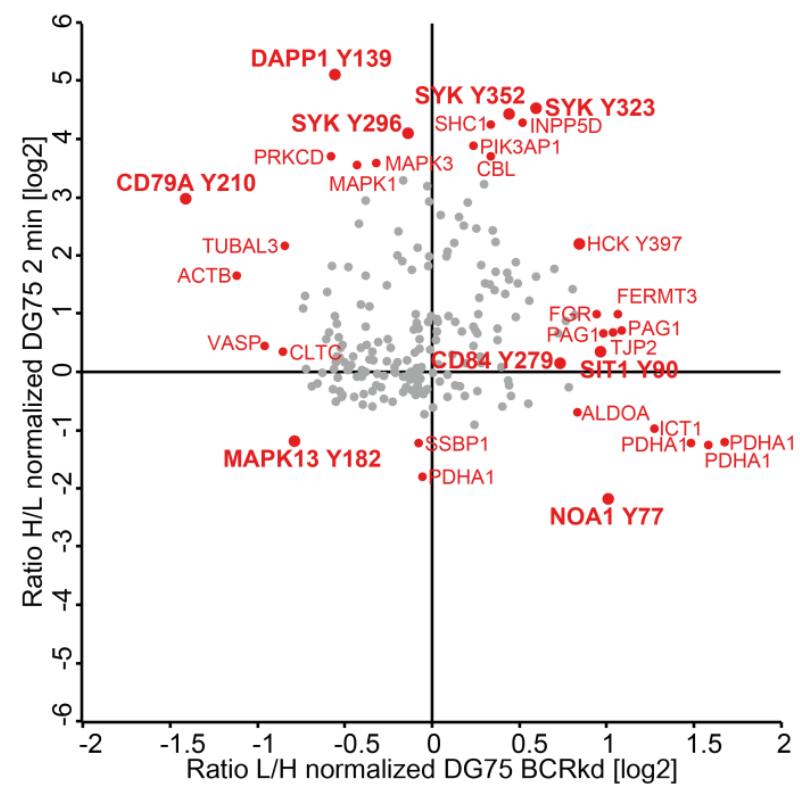

$\mathrm{C}$

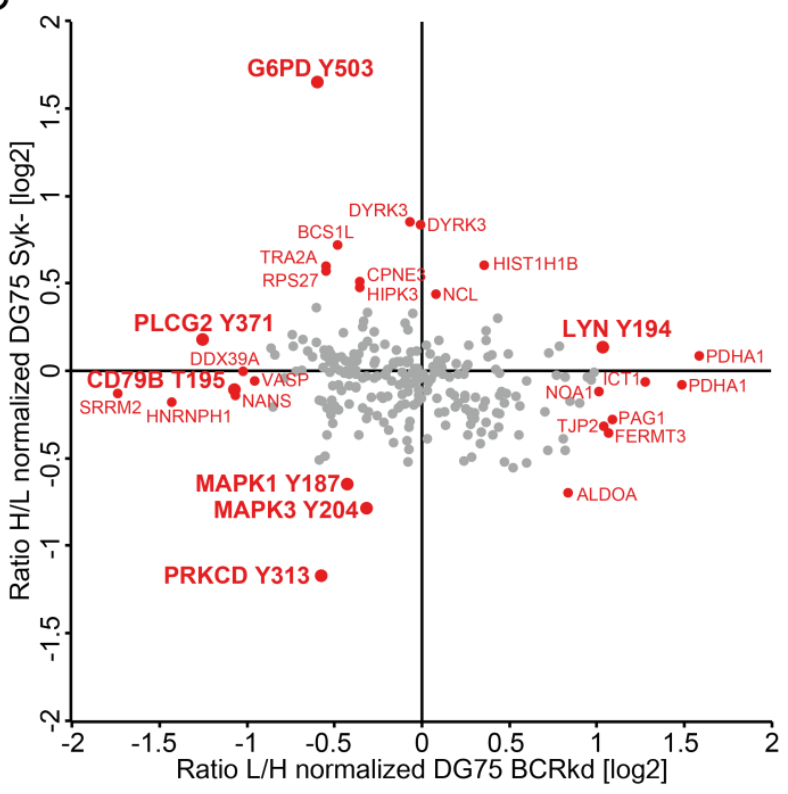

$E$

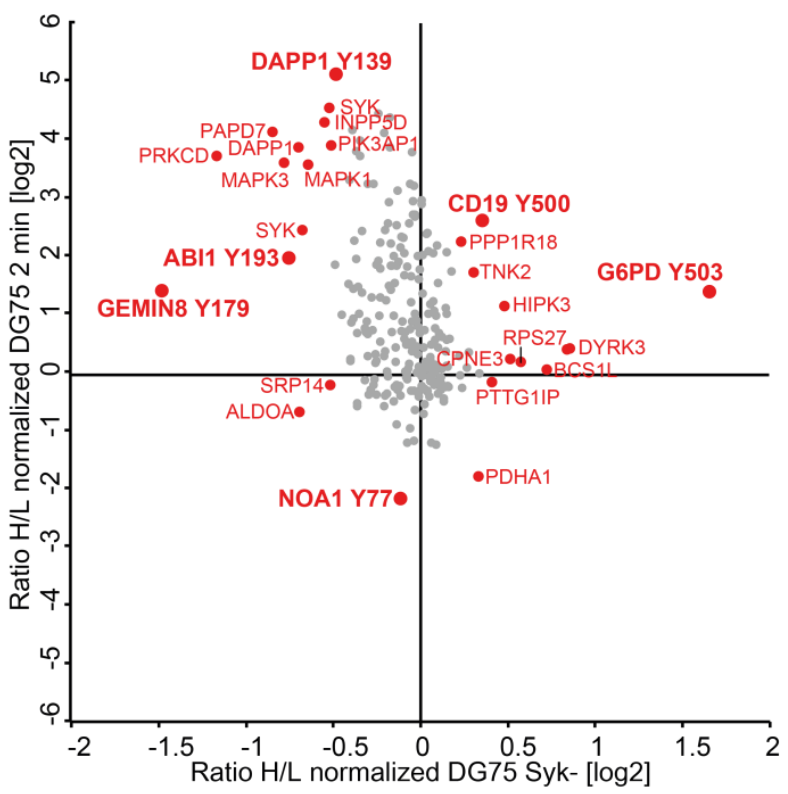

Figure 3.15: Antigen-independent and early antigen-dependent BCR signaling

(A) Protein expression ratios for CD79A and SYK derived from the antibody-based pY enrichment during BCR engagement (2, 5, 10 and $20 \mathrm{~min}$ ) and antigen-independent (BCR kd and Syk-) conditions. (B) Venn diagram displaying the number of individual and commonly quantified tonic signaling $p$-sites with BCR knockdown and SYK inhibition, respectively. (C) Scatter plot comparing the commonly quantified $\mathrm{p}$-sites in antigen-independent signaling. The $\mathrm{x}$-axis shows the normalized $\mathrm{L} / \mathrm{H}$ ratio ( $\log 2)$ of $\mathrm{p}$-sites in the BCR knockdown experiment and the $\mathrm{y}$-axis the normalized $\mathrm{H} / \mathrm{L}$ ratio $(\log 2)$ after pharmacological SYK inhibition. Outlier sites are marked in red and gene names are indicated. Sites specifically mentioned in the text are highlighted bold red and the phosphorylation position is indicated. (D) and (E) Scatter plot comparing commonly quantified p-sites immediately after antigen-mediated BCR stimulation (on the $y$-axis respectively) and in antigen-independent signaling (BCR kd in (D) and SYK inhibition in (E)). Outlier sites are marked in red and gene names are indicated. Sites specifically mentioned in the text are highlighted bold red and the position of phosphorylation is indicated. All sites identified and quantified in at least one experimental condition are listed in supplementary table S10. 
To determine differences between tonic signaling conditions and early phosphorylation events in antigen-dependent signaling derived from the respective pYome analyses, the SILAC ratios after 2 min of BCR engagement were plotted against the ratios after BCR knockdown and SYK inhibition (Figure 3.15 D and E). Compared to BCR stimulation, the tonic signals are as expected much weaker as can be seen by maximum upregulation of 1.7 and 1.7 and minimum downregulation of -1.6 and -1.2 of class I p-sites during BCR knockdown and SYK inhibition, respectively (Figure 3.15 D and E; supplementary table S10). Common BCR signaling effectors identified such as SYK, MAP-kinases, CBL, HCK, SHC1, DAPP1 and INPP5D (SHIP1) were detected as differentially phosphorylated in antigen-dependent and -independent signaling, respectively (Figure $3.15 \mathrm{D}$ and E). SYK Y323 and Y352, two auto-phosphorylation and activity regulating $p$-sites became phosphorylated after $2 \mathrm{~min}$ of BCR engagement as well as after CD79A knockdown (Y323: 4.5- and 0.6-fold, Y352: 4.4- and 0.4-fold; Figure 3.15 D; supplementary Table S10) (Deckert et al., 1998; Tsang et al., 2008). Another SYK p-site, Y296, was identified as phosphorylated after antigen stimulus but was not altered by the BCR knockdown (Figure 3.16 D; supplementary table S10). Y296 has been previously described in the context of BCR signaling, however, the functional role is not yet determined (Bohnenberger et al., 2011). MAPK13 Y182 (in isoform 2, corresponding to Y104 in isoform 1) within the activity determining TXY motif became dephosphorylated in both tonic signaling conditions (Figure 3.15 C and D, supplementary table S10) (Burack and Sturgill, 1997). DAPP1 (BAM32) Y139 was identified to be dephosphorylated after BCR knockdown whereas the site became phosphorylated after 2 min of BCR engagement (Figure 3.15 D; supplementary table S10). DAPP1 is a B cell-specific protein that becomes recruited by its PH domain to the cell membrane after $B$ cell engagement followed by phosphorylation of $\mathrm{Y} 139$ by PI3K, LYN, LCK or SRC (Allam et al., 2004; Anderson et al., 2000; Montalvo et al., 2000; Marshall et al., 2000). DAPP1 is of special interest as is has been functionally associated with cytoskeleton regulator remodelling after BCR stimulation (Allam et al., 2004; Anderson et al., 2000). Furthermore, it has been also linked to the activation of the MAP-kinase pathway (Allam et al., 2004). NOA1 is a mitochondrial GTPase that became dephosphorylated at Y77 after 2 min of BCR stimulation whereas BCR knockdown lead to an increase in phosphorylation (Figure 3.15 D) (Kolanczyk et al., 2010). During SYK inhibition, the phosphorylation status of NOA1 Y77 did not change (Figure 3.15 D and E; supplementary table S10). SIT1 Y90 became phosphorylated after BCR knockdown (1-fold; Figure 3.15 D; supplementary table 10) but was not found to be 
regulated after BCR stimulation. SIT1 is involved in negative regulation of TCR signaling and phosphorylation of Y90 is described to provide an interaction site for GRB2 (Marie-Cardine et al., 1999). CD84 Y279 was upregulated after BCR knockdown (0.7-fold) while the p-site was not regulated during the BCR stimulation time course and the $\mathrm{p}$-site but could not be identified after SYK inhibition (Figure $3.15 \mathrm{D}$; supplementary table S10). CD84 is a receptor of the Igsuperfamily, expressed on immune cells (Tangye et al., 2003; la Fuente et al., 1997). Phosphorylation of Y279 (in isoform 3, corresponding to Y296 in isoform 1) is associated with protein interaction by providing a SH2 domain binding site (Schweigel et al., 2013; Tangye et al., 2003). CD19 Y500 was identified as phosphorylated after early BCR stimulation as well slightly increased after SYK-mediated BCR signaling ablation (2.6- and 0.4-fold, respectively) while the site was not identified after BCR knockdown (Figure $3.15 \mathrm{E}$; supplementary table S10). CD19 is a BCR co-receptor that lowers the BCR activation threshold and is involved in BCR-dependent PI3K-signaling (Carter and Fearon, 1992; Otero et al., 2000). Phosphorylated CD19 Y500 is involved in the activity amplification of the Src kinase LYN (Fujimoto et al., 2000). During the process, phosphorylated Y500 provides an interaction platform for other LYN proteins that phosphorylates further tyrosine residues leading to LYN activation (Y500 corresponds to Y482 in Fujimoto et al., 2000).

The Glucose-6-phosphate 1-dehydrogenase (G6PD) is a metabolic enzyme that was found to be phosphorylated at position Y503 after 2 min of BCR stimulation and SYK inhibition whereas the site became dephosphorylated after BCR knockdown (1.4- and 1.7-fold increase and 0.6fold decrease, respectively; Figure $3.15 \mathrm{C}$ and E, supplementary table S10). The p-site Y503 is in close proximity to the C-terminus of the enzyme and has been identified in many cell lines and disease models (listed in the PSP database) but is functionally not further described in the literature. GEMIN8 Y179 phosphorylation was found to be increased after BCR activation, whereas SYK inhibition leads to a decrease in phosphorylation (1.3-fold increase and 1.4-fold decrease, respectively; Figure $3.15 \mathrm{E}$; supplementary table S10). GEMIN8 is a catalytic protein in the SMN complex required for the assembly of snRNPs. No functional p-site information is available (Carissimi et al., 2006; Pellizzoni, 2007). The Abl interactor 1 (ABI1) phosphorylation on Y193 (corresponding to Y198 in isoform 1) was identified with a 1.9-fold increased after 2 min of BCR stimulation and 0.8-fold decreased after SYK inhibition (Figure $3.15 \mathrm{E}$; supplementary table S10). ABI1 Y193 could only be identified after 2 and 5 min of BCR engagement, after SYK inhibition but not during CD79A knockdown. ABI1 is known to be 
involved in cytoskeleton regulation, however, functional information about the $p$-site is not deposited in databases (supplementary table S10) (Leng et al., 2005; Stradal et al., 2001).

To determine globally substantial differences between the time-resolved BCR stimulation and antigen-independent signaling in DG75 cells, the p-site SILAC ratios of the BCR stimulation time course were compared to both tonic signaling conditions on the pYome level. In total, 203 class I p-sites (192 pY sites) were commonly quantified in the antigen-dependent time course (in at least two time points) and the antigen-independent conditions (Figure 3.15 A; supplementary table S10). Unsupervised hierarchical clustering was performed and the common p-sites could be separated in three main cluster profiles (Figure $3.15 \mathrm{~A}$ ). From the largest cluster (red row tree), a subcluster (bracket) consisting of $41 \mathrm{p}$-sites (38 pY sites) derived from 31 effector proteins was extracted representing a set of highly differential $p$ sites between the two experimental settings (heat map and cluster representation in Figure 3.16; supplementary table S10). The cluster represents $p$-sites that became phosphorylated after BCR engagement and showed no changes or became dephosphorylated during tonic signaling (Figure 3.16 A). The effector proteins together with their $p$-sites were subjected to visualization with PhosphoPath to complement the dataset with protein-protein interaction, kinase-substrate and signaling pathway information (Figure 3.16 B) (Raaijmakers et al., 2015). Pathway enrichment analysis of the involved effector proteins included 'B-cell receptor signaling pathway' and 'IL-3 signaling pathway' as the most enriched signaling pathways (according to Wikipathways; data not shown). 


\section{Results}

A

203 sites

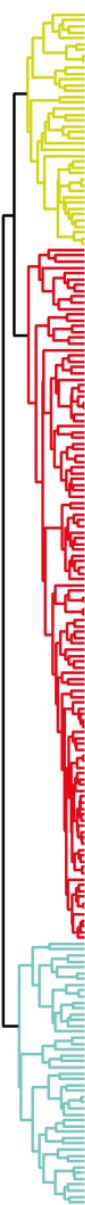

B

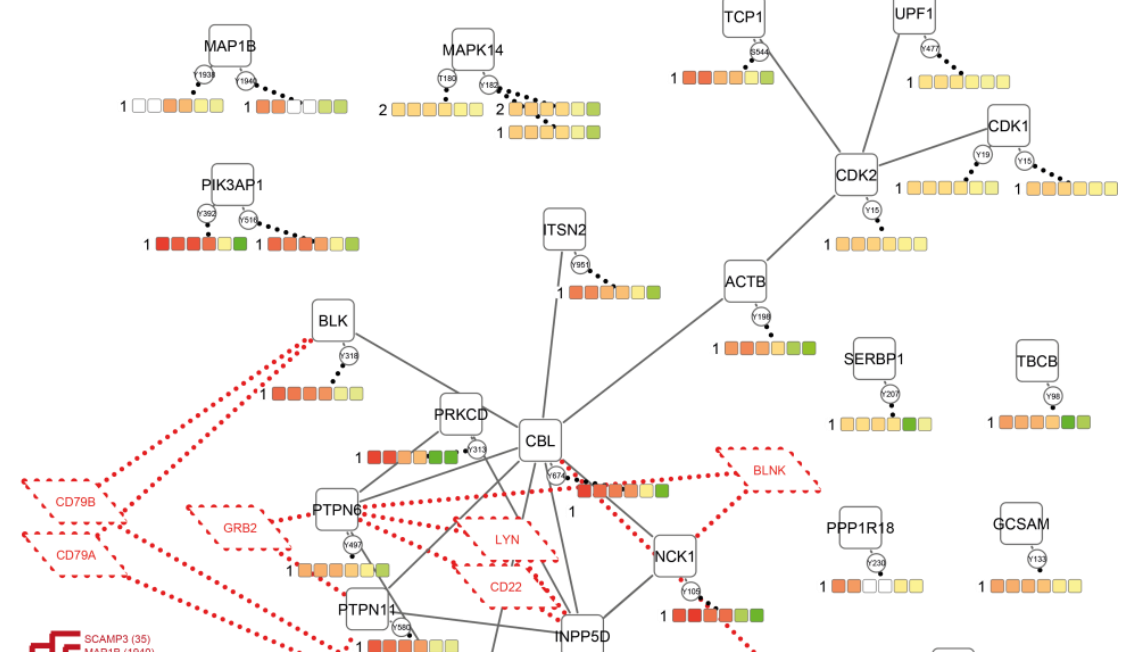

antigen-dependent tonic

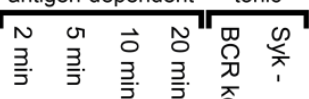

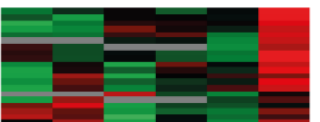

\section{(1)}
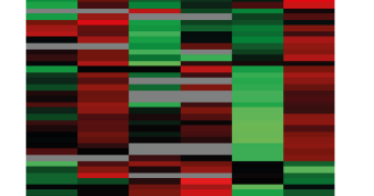
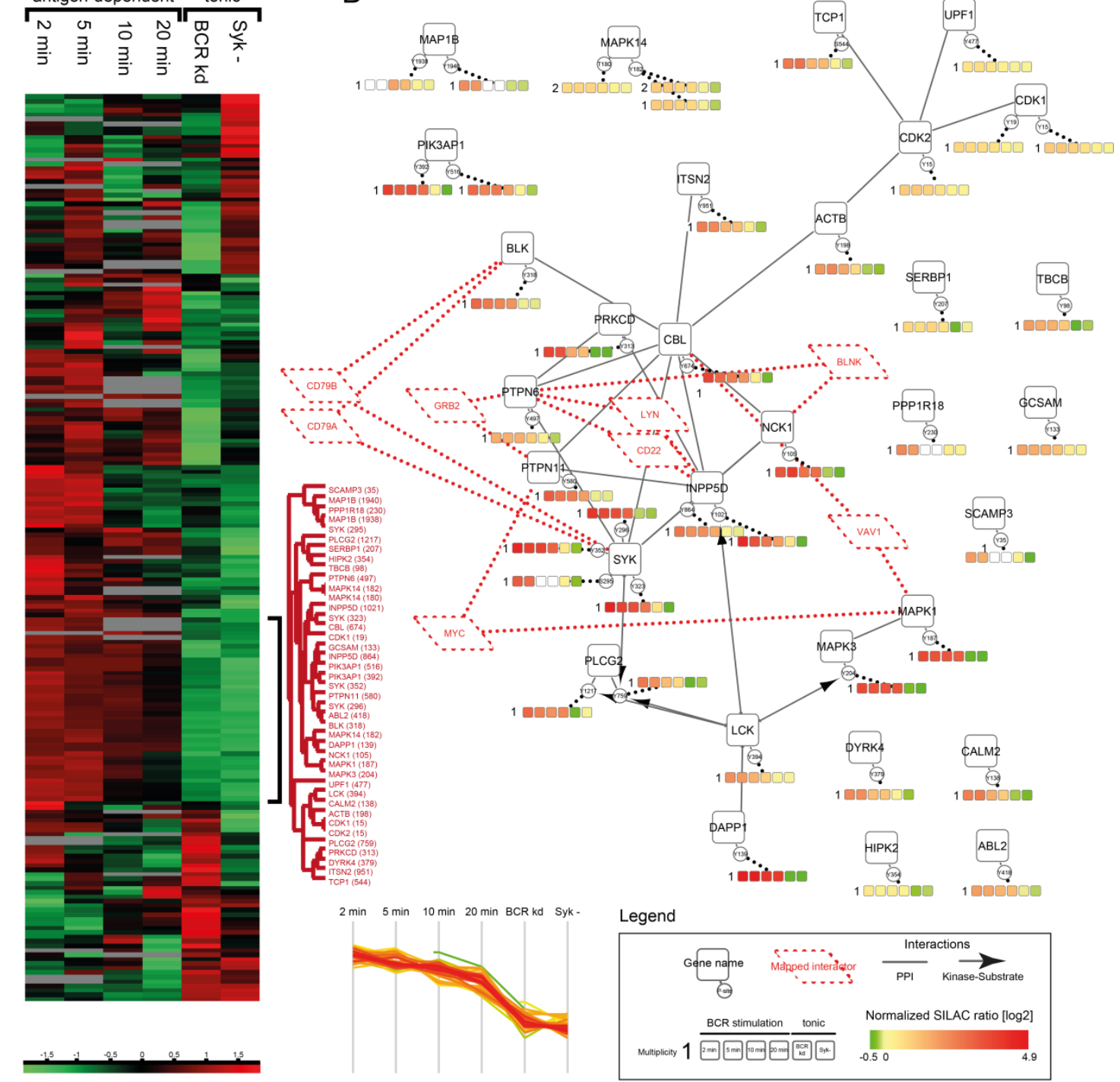

Figure 3.16: Differential regulation in antigen-dependent and tonic BCR signaling

(A) Heat map of 203 common p-sites quantified in the antigen-dependent time course (in at least two of four time points) and the antigen-independent experiments. Normalized SILAC ratios (log2) were Z-scored and subjected to hierarchical cluster analysis. Z-scored p-site SILAC ratios (row) of all experimental conditions (column) are colored according to the color scale at the bottom. The dataset was split in three distinct clusters and additionally, the most differential sites were manually extracted. The extracted row tree, gene names, the position of the phosphorylated amino acid (in brackets) and the profile of the cluster are shown right to the heat map. (B) The extracted, most differential sites (see A) were visualized by the Cytoscape App PhosphoPath (Raaijmakers et al., 2015). Proteins were subjected to protein-protein and additionally p-sites to kinase-substrate interaction analysis (according to BioGRID, STRING and PSP, respectively). Proteins are represented by a rounded rectangle, $p$-sites by ellipses. The quantitative SILAC ratio information across all time points and conditions is plotted below each $p$-site. Each rectangle represents a different condition and the color filling represents quantitative values according to the color code (see legend), sites not quantified are coloured in white. A dashed diamond node indicates mapped interactors from (BioGRID or STRING) that were not directly extracted from the set of common sites or identified from the analysis. Gene names, the position and amino acid of the modification are indicated within the respective nodes. PPI interactions are represented by a line, kinase-substrate interactions by an arrow. PPI: Protein-protein interaction. P-sites positions indicated in the figure dependent on the respective protein isoform listed in the supplementary table. All common and differentially regulated $\mathrm{p}$-sites are listed in supplementary table S10.

19 of the 31 effector proteins were involved in protein-protein and kinase-substrate

interactions as mapped by BioGRID, STRING and PSP implemented in PhosphoPath (Figure 
3.16 B) (Raaijmakers et al., 2015). The connected proteins are most commonly known as BCR signaling effectors such as CBL, BLK, SYK, INPP5D (SHIP1), PLCG2, LCK, MAPK1 and MAPK3. The network was further expanded with known interactors (CD79A/B, GRB2, BLNK, VAV1 and MYC) by PSP and BioGRID database mapping (indicated as dashed red diamond-shaped nodes) which were not extracted as regulated or not quantified in either of the conditions (Figure 3.16 B). The most differential phosphorylated sites between the two groups of antigenactivated and tonic signaling were PRKCD Y313, MAPK1 Y187, MAPK3 Y204, PLCG2 Y759, NCK Y105, DAPP1 Y139, TBCB Y96 and CALM2 Y138 of which several were described above (Figure 3.16; Figure 3.16 C, D and E; supplementary table S10). In the following, further p-sites that appeared to be differentially regulated in antigen-dependent and -independent signaling will be described and set in a functional context. The SYK p-sites, S295 and the autophosphorylation and activity-determining p-sites Y296, Y323, Y352 could be commonly quantified during BCR stimulation and tonic BCR signaling (Mócsai et al., 2010 refers to a six amino acids shorter form of Syk). Phosphorylation of the before mentioned sites increased during $B C R$ stimulation whereas in tonic signaling conditions, phosphorylation stayed at similar levels or was decreased compared to the ground state, respectively (Figure $3.16 \mathrm{~B}$ ). Phosphorylated SYK S295 could be quantified for the early time points of BCR stimulation (2 and $5 \mathrm{~min}$ ) while being absent at the late time points (10 and $20 \mathrm{~min}$ ). In the tonic signaling conditions, pS295 levels were only slightly altered compared to the ground state $(0.3$-fold increase and 0.3-fold decrease during BCR knockdown and SYK inhibition, respectively; Figure 3.16 B). Phosphorylation of S295 is mediated by CHK1 and primes SYK for degradation via the ubiquitin/proteasome pathway (Hong et al., 2012). The TXY motif in MAPK1 and MAPK3 became heavily phosphorylated at both positions simultaneously after BCR engagement reflecting kinase activation but could not be quantified in tonic signaling (supplementary Table S2 and S10). However, MAPK1 Y178 and MAPK1 Y204 (in isoform 1) were quantified as dephosphorylated during tonic signaling and SYK inhibition (0.4- and 0.3-fold and 0.6- and 0.8fold decrease, respectively; Figure 3.16 B). ABL2 (Abelson tyrosine-protein kinase 2) became phosphorylated at position Y418 (in isoform 10, corresponding to ABL2 Y439 in isoform 1) during the whole time course of BCR stimulation. No phosphorylation change could be observed under BCR knockdown and only a slight decrease was quantified after SYK inhibition (Figure 3.16 B; supplementary table S10). ABL2 is a non-receptor tyrosine kinase involved in a multitude of biological processes and signaling pathways. ABL2 Y439 is located in the 
activation loop and its phosphorylation is mediated by autocatalysis or by Src-type kinases such as HCK (Tanis et al., 2003). Furthermore, Y418 phosphorylation leads to ABL2 degradation (Cao et al., 2005). The CALM2 (Calmodulin) p-site Y138 was identified as phosphorylated after BCR engagement and was found to be dephosphorylated during BCR knockdown and SYK inhibition (0.2- and 0.3-fold decreased, respectively; Figure 3.16 B; supplementary table S10). The $p$-site is not further described or characterized but lies within the EF hand domain required for calcium binding. PIK3AP (BCAP) Y516 (in isoform 2, corresponding to Y694 in isoform 1) became phosphorylated after BCR engagement (3-fold after $2 \mathrm{~min}$ ) and is not regulated after BCR knockdown and after SYK inhibition (supplementary table S10). PIK3AP1 is an adapter protein connecting BCR signaling to PI3K-kinase signaling. The site is one of five conserved tyrosine residues and is a substrate of $A B L$ (Maruoka et al., 2005).

Overall, analysis of tonic BCR signaling has not been performed in a global manner. The analysis revealed many interesting $\mathrm{p}$-sites of which only a few were mentioned in this work. The provided dataset presents a basis for further functional characterization of phosphorylation sites and effector proteins involved in tonic BCR signaling. 


\subsection{Acute myeloid leukemia}

The phosphoproteomic analysis of Burkitt's lymphoma under various experimental conditions described in chapter 3.1 provides insights into intracellular signaling mechanisms mediated by phosphorylation. Especially antibody-based phosphotyrosine enrichment in different experimental conditions proofed to be a valuable methodology to study signaling. Therefore the comprehensive phosphoproteomic approach used for the analysis of Burkitt's lymphoma was applied to another hematological disorder, namely acute myeloid leukemia ( $A M L)$. AML is derived from malignant transformed hematopoietic cells. Cells are highly undifferentiated and show a variety of mutational backgrounds in patients that lead to different therapeutic outcomes (The Cancer Genome Atlas Research Network, 2013) . This makes the identification of common targets and the application of generalized therapeutic strategies difficult. In previous studies, the tyrosine kinases SYK and BTK were found to be constitutively activated in AML and therefore were considered as potential drug targets (Hahn et al., 2009; Rushworth et al., 2013; Zaitseva et al., 2014). Both kinases are throughout described in the context of BCR signaling and BTK inhibition is already a standard therapeutic treatment in chronic lymphatic leukemia (Ponader et al., 2011; de Rooij et al., 2012). It could be shown that the pharmacological inhibition of SYK in AML cells results in either apoptosis or differentiation into myeloid effector cells (Hahn et al., 2009). The SYK activation mechanism, how it drives oncogenic AML blast proliferation, the identification of SYK-dependent phosphorylation sites and the analysis of SYK interaction partners was already studied by Oellerich et al., 2013. Furthermore was BTK inhibition in AML cells reported to reduce AML blast proliferation and to increase the cytotoxic effects of standard chemotherapeutics (Rushworth et al., 2013). However, the underlying mechanisms i.e. the identity of the upstream regulators, the downstream signaling partners and pathways involved are not known to date. Point mutations in the tyrosine kinase and an internal tandem duplicaton (ITD) in the juxtamembrane domain in the FMS-like tyrosine kinase 3 (FLT3) are one of the most common mutational events in AML (Nakao et al., 1996; The Cancer Genome Atlas Research Network, 2013). Therefore, the role of BTK signaling in the context of different mutational backgrounds was investigated. The cell lines MV4-11 with a FLT3-ITD-positive and KG1 with a FLT3-ITD-negative background were representatively chosen for the comparative study. The generalized BTK expression and the BTK activation status, represented by the phosphorylation of the activating tyrosine residue at position Y223, were verified in MV4-11 and KG1 by Oellerich et al., 2015. BTK interactors in 
MV4-11 and KG1 were identified by knockdown experiments in combination with SILAC-based quantitative mass spectrometry approaches as described in Oellerich et al., 2015. Based on the assumption that BTK activation in FLT3-ITD-positive AML cells is dependent on FLT3-ITD activity, BTK was found in the FLT3 signalosome of MV4-11 (Oellerich et al., 2015). The BTK signalosome in KG1 identified the CpG oligonucleotide recognizing Toll-like receptor 9 (TLR9) as interaction partner (Oellerich et al., 2015). According to previous studies, an interaction between TLRs and BTK is already described in B cells and macrophages (Kubo et al., 2009; Vijayan and Baumgart, 2011). The interaction of BTK and TLR9 and of FLT3-ITD and BTK in KG1 and MV4-11 was further validated as described in Oellerich et al., 2015.

\subsubsection{BTK-dependent phosphoproteome networks in AML}

BTK was found expressed, active and moreover involved in the signalosomes as described above in KG1 and MV4-11 AML cell lines (Oellerich et al., 2015). The aim of the following experiments was to find out whether BTK inhibition with ibrutinib is also a suitable treatment strategy in AML and furthermore to describe the effects and mechanism of its signal inhibition properties. Ibrutinib is an irreversible BTK inhibitor already established in the treatment of other B lymphoid malignancies such as chronic lymphatic leukemia or mantle-cell lymphoma (Byrd et al., 2014; Wang et al., 2013). The application effects of ibrutinib in KG1 and MV4-11 were investigated on the phosphoproteomic and protein expression level. To gain insights into signaling events downstream of BTK, heavy SILAC labeled cells treated with ibrutinib were compared to untreated light-labeled cells (Figure 3.17 A). A dual quantitative phosphoproteomic analyses was performed consisting first of antibody-based phosphorylated tyrosine enrichment ( $p Y o m e$ ) and second of $\mathrm{SCX}$ in combination with $\mathrm{TiO}_{2}$ affinity purification (global phosphoproteome) followed by LC-MS/MS measurement. After antibody-based phosphotyrosine enrichment in KG1, 875 class I p-sites (726 tyrosine sites) and in MV4-11, 571 class I p-sites (476 tyrosine sites) could be quantified, respectively. 316 p-sites were commonly identified in both cell lines (Figure 3.17 B; supplementary table S11). 233 p-sites of those common sites showed a SILAC ratio difference $\leq|0.5|$ indicating concordant behaviour 
(regulated as well as non-regulated) and 83 common sites had a SILAC ratio difference $\geq|0.5|$

(regulated and non-regulated; Figure $3.17 \mathrm{C}$ ).
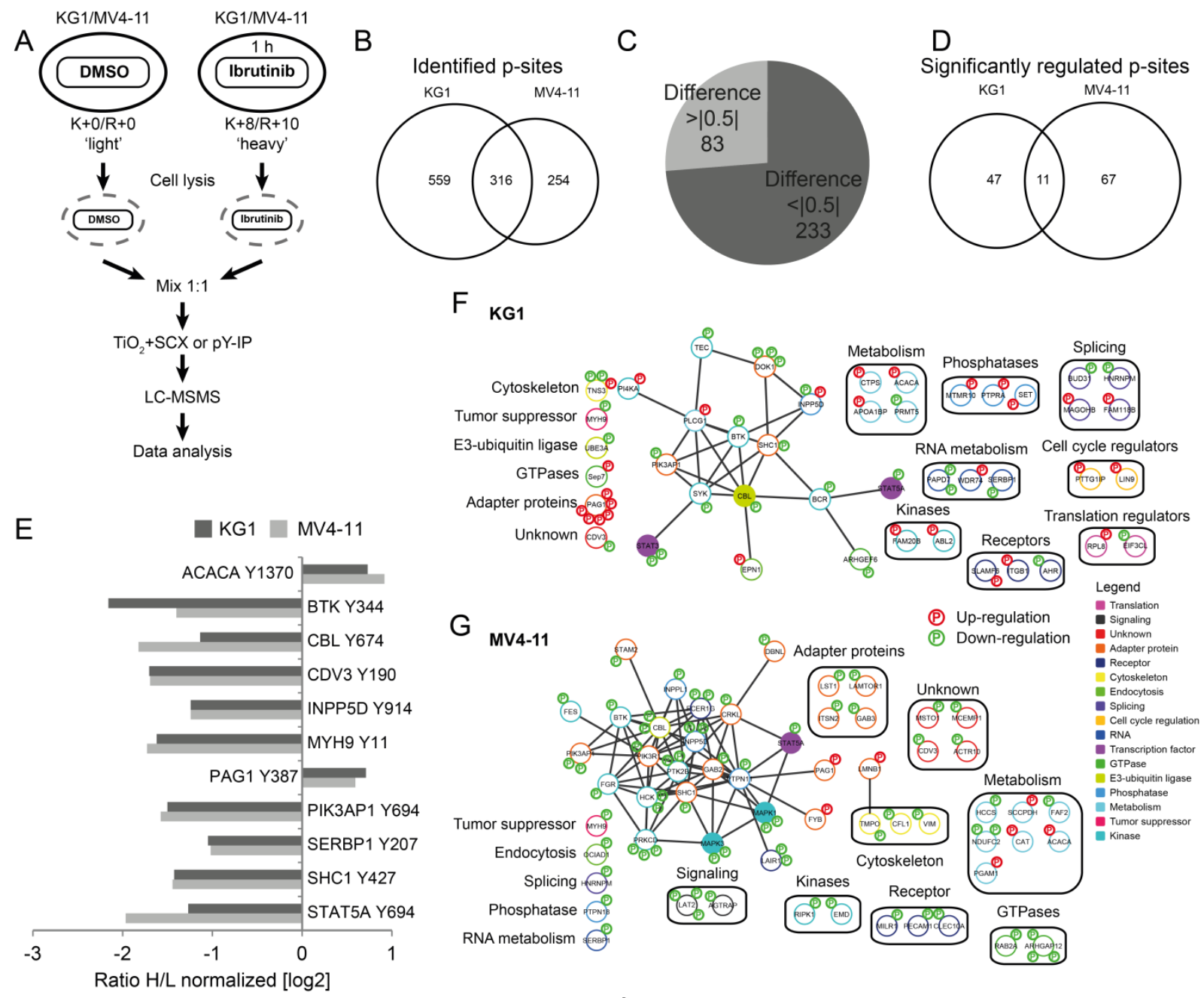

$\mathrm{H}$
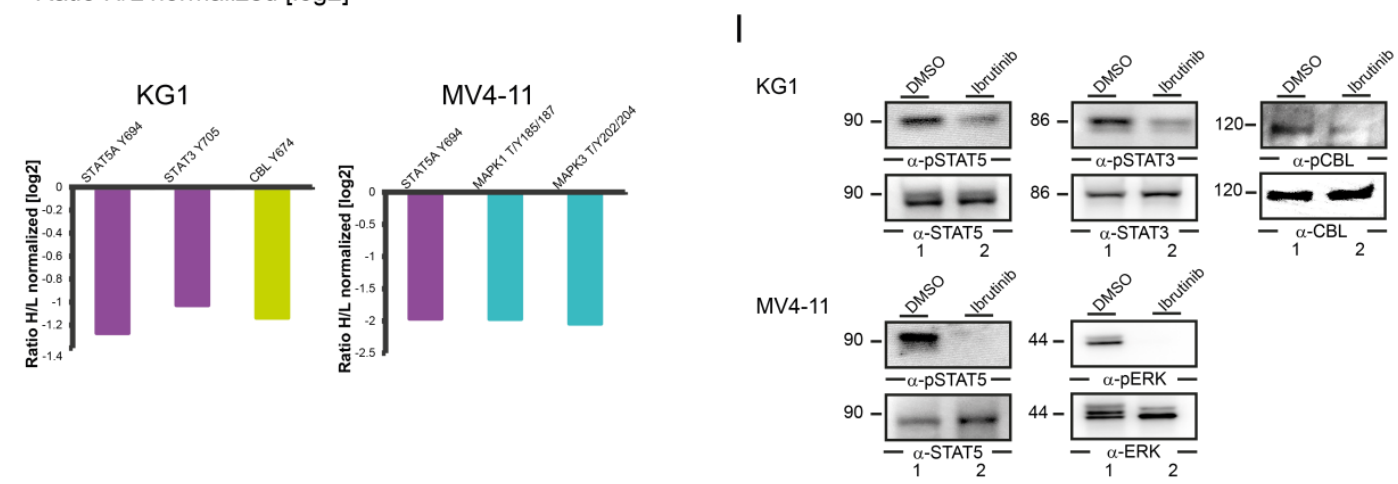

Figure 3.17: Phosphorylated tyrosine dynamics after BTK inhibition

(A) Experimental design of the SILAC-based BTK inhibition pYome analysis. Heavy-labeled cells were treated for one hour with ibrutinib, light-labeled cells were treated with DMSO as a control. Cells were lysed, proteins were mixed at a 1:1 ratio, run on a SDS-PAGE gel and digested with trypsin. Peptides were immunopurified for phosphorylated tyrosine residues followed by mass spectrometric analysis (B) Venn diagram displaying the number of exclusively and commonly quantified class I p-sites for KG1 and MV4-11 cells, respectively. (C) The pie chart shows the relative abundance of $p$-sites with a SILAC ratio differences smaller or greater than $|0.5|$ of commonly quantified $p$-sites. (D) The Venn diagram shows the number of exclusively and commonly regulated $\mathrm{p}$-sites (primarily $\mathrm{pY}$ sites) after $1 \mathrm{~h}$ of ibrutinib treatment for KG1 and MV4-11, respectively. The regulation interval was set outside to ]-1;0.5[. (E) The bar diagram shows the normalized SILAC ratios H/L (log2) of the regulated p-sites found in both, KG1 and MV4-11 cells. (F) and (G) Signaling networks contain proteins that were identified as being phosphorylated (red P) or dephosphorylated (green P) on serine, threonine or tyrosine residues in response to BTK 
inhibition. Proteins were grouped according to their known protein-protein interaction status listed in the STRING database. The assigned protein functions were derived by manual annotation using UniProt, PhosphoSitePlus and PubMed databases and are visualized by color coding (see legend). The nodes of STAT5A, STAT3, CBL, and MAPK1/3 are highlighted by color fill. (H) Normalized SILAC ratios of the regulated phosphotyrosines sites STAT5A Y694, STAT3 Y705, CBL Y674 in KG1 and STAT5A Y694, MAPK1 T185/Y187, MAPK3 T202/Y204 in MV4-11 cells. The color coding of the bars refers to the functional groups of the proteins highlighted (filled nodes) in (F) and (G). (I) Cleared cellular lysates, derived from KG1 and MV4-11 cells that had either been left untreated or been treated with $500 \mathrm{nM}$ ibrutinib for one hour were subjected to immunoblotting using antibodies against phosphorylated STAT5, ERK, STAT3, FOXO1 and C-CBL, respectively. Cell culture of KG1 and MV4-11, ibrutinib treatment as shown in (A), and western blot analysis shown in (I) was performed at the Department of Internal Medicine, Hematology/Oncology at the University Hospital Frankfurt/Main as part of the collaboration mentioned in Table 12. The figure is adapted from Oellerich et al., 2015. All identified and regulated p-sites for KG1 and MV4-11 cell lines are listed in supplementary table S11.

In KG1, 58 p-sites and in MV4-11 78 p-sites were found regulated (Figure 3.17 D; supplementary table S11). Eleven tyrosine $p$-sites derived from distinct proteins were found regulated in both cell lines (Figure 3.17 D). Those common, regulated sites showed overall concordant regulation behaviour: nine p-sites were dephosphorylated while ACACA Y1370 and PAG1 Y387 were phosphorylated after BTK inhibition (Figure 3.17 E). The BTK SH2 domain p-site Y344 was among those sites found to be dephosphorylated after ibrutinib treatment. According to database information, no functional process, upstream kinase or regulatory behavior could be associated yet with this site (according to PSP and UniProt). To examine in detail how the identified BTK effector proteins are integrated into signalosomes and signaling cascades, protein network analyses (Figure 3.17 F and G) were generated based on the differentially phosphorylated proteins (Figure 3.17 D). The identified BTK effectors proteins fall into a multitude of functional protein classes, including cell cycle and metabolism regulators. Among the regulated effectors, STAT5A/B, CBL and MAPK1/3 were previously described in the context of AML cell survival and proliferation (Hayakawa et al., 2000; Sargin et al., 2007). The p-sites on the mentioned proteins, STAT5A Y694, STAT3 Y705, CBL Y674 in KG1 and STAT5A Y694, MAPK1 T185/Y187, MAPK3 T202/Y204 in MV4-11 cells were dephosphorylated after ibrutinib treatment (Figure 3.17 F, G and H). STAT5A Y694 and CBL Y674 are putative targets of BTK (according to NetworKIN; data not shown). The MS-based phosphorylation status of the before mentioned proteins after ibrutinib treatment was additionally verified by immunoblotting (Figure $3.17 \mathrm{I}$ ).

For the determination of changes in the global phosphoproteome after BTK inhibition i.e. mainly phosphorylation changes on serine and threonine residues, the same experimental setup as described above for the pYome was applied (Figure 3.17 A). In KG1 cells 2891 class I p-sites and in MV4-11 cells 2011 class I p-sites could be quantified of which 186 and 139 psites where considered to be regulated, respectively (highlighted red, Figure 3.18 A and B; 
supplementary table S12). To determine biological meaningful p-sites, the ones with SILAC ratios $<-0.5$ or $>0.5$ in KG1 and MV4-11 were selected, respectively. This resulted in $42 \mathrm{p}$-sites for KG1 and 26 p-sites for MV4-11 (highlighted data points represent individual p-sites, gene names are indicated; Figure $3.18 \mathrm{~A}$ and $\mathrm{B})$.
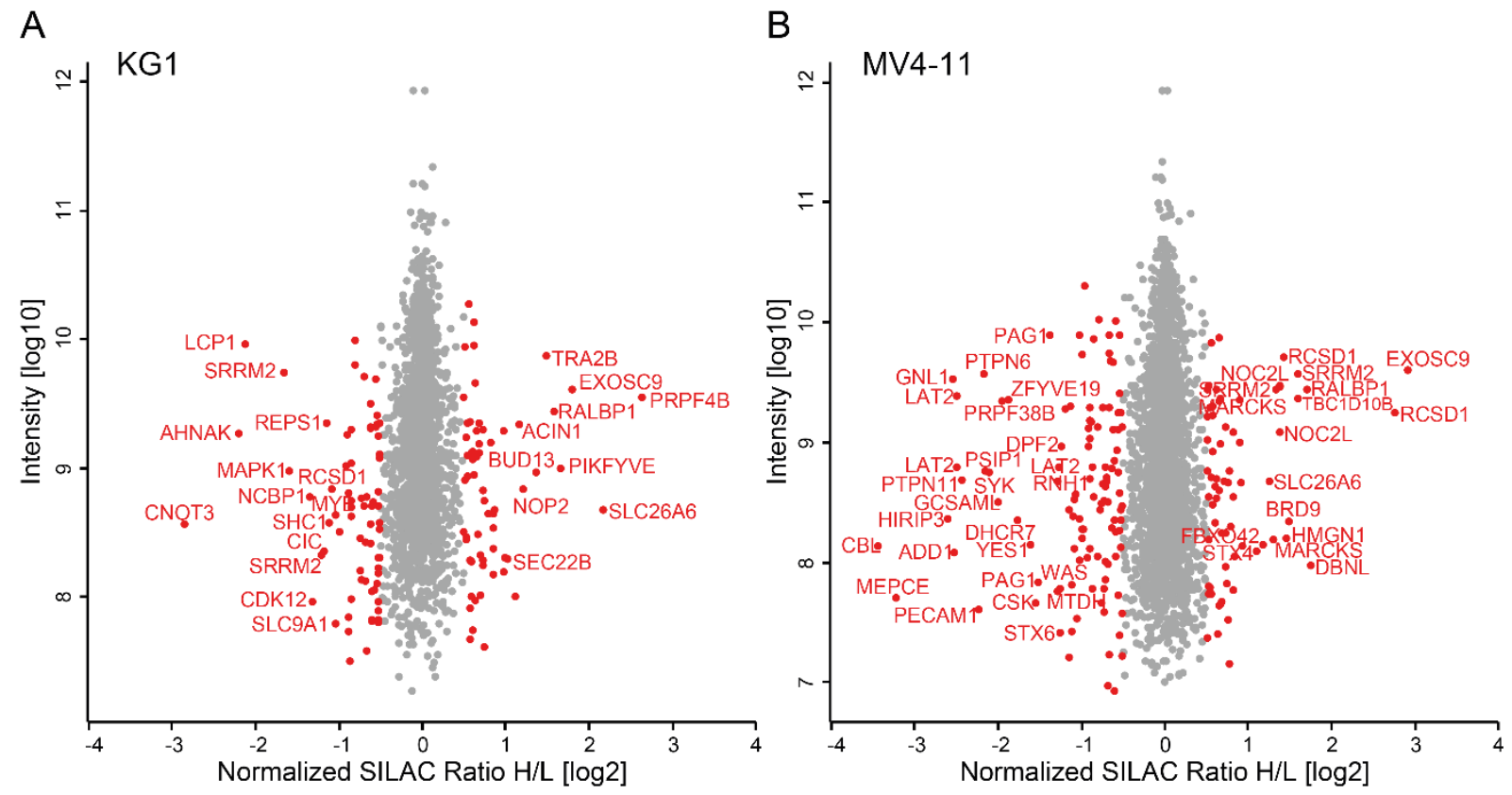

Figure 3.18: Global phosphoproteomic analysis after BTK inhibition in AML

(A) and (B) Scatter plots of the distribution of normalized $p$-site H/L SILAC ratios ( $x$-axis) versus their intensity ( $y$-axis) after one hour of BTK inhibition with ibrutinib in KG1 (A) or MV4-11 (B). P-sites with H/L SILAC ratios outside the range ]-0.5;0.5[ were considered to be regulated and are highlighted in red. For the most prominent outlier $p$-sites (for KG1 $<-1.2$ or $>1$; for MV4-11 $<-1$ or $>1$ ) the gene names are indicated additionally. Cell lines were cultured and treated at the Department of Internal Medicine, Hematology/Oncology at the University Hospital Frankfurt/Main as part of the collaboration mentioned in Table 12. Regulated p-sites derived from the global phosphoproteomic analysis of KG1 and MV4-11 cells are listed in supplementary table S12.

To not only highlight individual differences between the two cell lines, the quantified $p$-sites were compared to each other. $1399 \mathrm{p}$-sites were quantified in both cell lines (data not shown) of which 19 common p-sites on 16 effector proteins were regulated in both cell lines (Figure 3.19). Both, the SHC-transforming protein 1 (SHC1) Y317 (in isoform 2; corresponding to Y427 in isoform 1) and the tumour protein TPD52 (D52) S136 (in isoform 2; corresponding to S176 in isoform 1) were quantified as dephosphorylated after BTK inhibition. The former site provides a binding site for GRB2 and is described in the context of a multitude of cellular processes such as cell differentiation, cytoskeleton, apoptosis, and different diseased tissues (Patrussi et al., 2005). TPD52 S136 phosphorylation is associated with plasma membrane localization and lysosomal membrane trafficking (Thomas et al., 2010; 2009). 


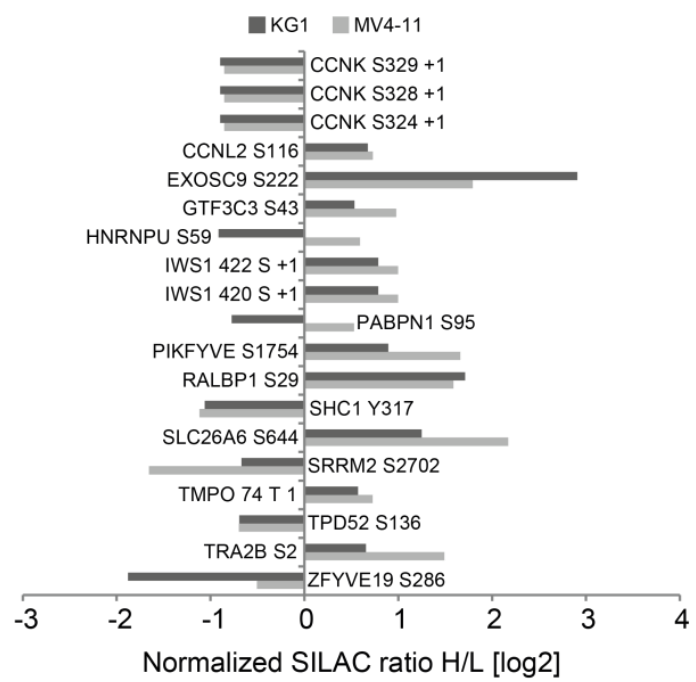

Figure 3.19: Common regulated sites in KG1 and MV4-11

Bar diagram showing the normalized H/L SILAC ratios for the 19 common regulated p-sites derived from the global phosphoproteome. Quantitation ratios of common p-sites shown above are listed in supplementary table S12.

\subsubsection{BTK-dependent protein expression levels}

Changes on the protein expression levels are expected to require a longer time compared to changes on the PTM level like phosphorylation. Therefore the time scale for the protein expression analysis was performed at a wider range with $6 \mathrm{~h}$ and $24 \mathrm{~h}$ of ibrutinib treatment for heavy-labeled KG1 and MV4-11 cells, respectively. These were compared to their lightlabeled, untreated counterparts (Figure 3.20 A). FLT3 and TLR9 were associated with malignant BTK activation and CBL, STAT5A/B and MAPK1/3 were previously described as differentially phosphorylated in the pYome analysis in section 3.2.1 and therefore presumably play a role in AML signaling. Protein expression changes of BTK, FLT3 and further interesting candidates like CBL, STAT5A/B and MAPK1/3 were extracted from the proteomic dataset and are shown in Figure 3.20 B (supplementary table S12). TLR9 was identified as putative BTK activator in the FLT3-ITD-negative cell line KG1 but could not be quantified on the proteomic level after BTK inhibition in KG1 and MV4-11, respectively. BTK could be quantified in the two cell models for both ibrutinib treatment durations (Figure $3.20 \mathrm{~B}$; supplementary table S12). BTK levels were slightly elevated (normalized SILAC ratio of 0.64 ) in KG1 after $24 \mathrm{~h}$ of BTK inhibition whereas only small changes could be monitored in MV4-11 (Figure 3.20 B; supplementary table S12). The mutated and therefore constitutive active receptor tyrosine kinase FLT3 was quantified in MV4-11 with slightly increased expression levels after $6 \mathrm{~h}$ of ibrutinib treatment (0.48-fold) and was 1 -fold increased on a log2 scale after $24 \mathrm{~h}$ (Figure 3.20 B). The protein expression of the phosphorylation regulated E3-ubiquitin-ligase CBL was not 
changed in both cell lines (Figure $3.20 \mathrm{~B}$; supplementary table S12). The transcription factor

STAT5B was upregulated after $24 \mathrm{~h}$ of ibrutinib treatment in KG1 and MV4-11 cells whereas STAT5A was only upregulated after $24 \mathrm{~h}$ in KG1 but not in MV4-11 (Figure $3.20 \mathrm{~B}$; supplementary table S12). MAPK1 and 3 could only be quantified in MV4-11 and did not show regulation behaviour (Figure $3.20 \mathrm{~B}$ ).
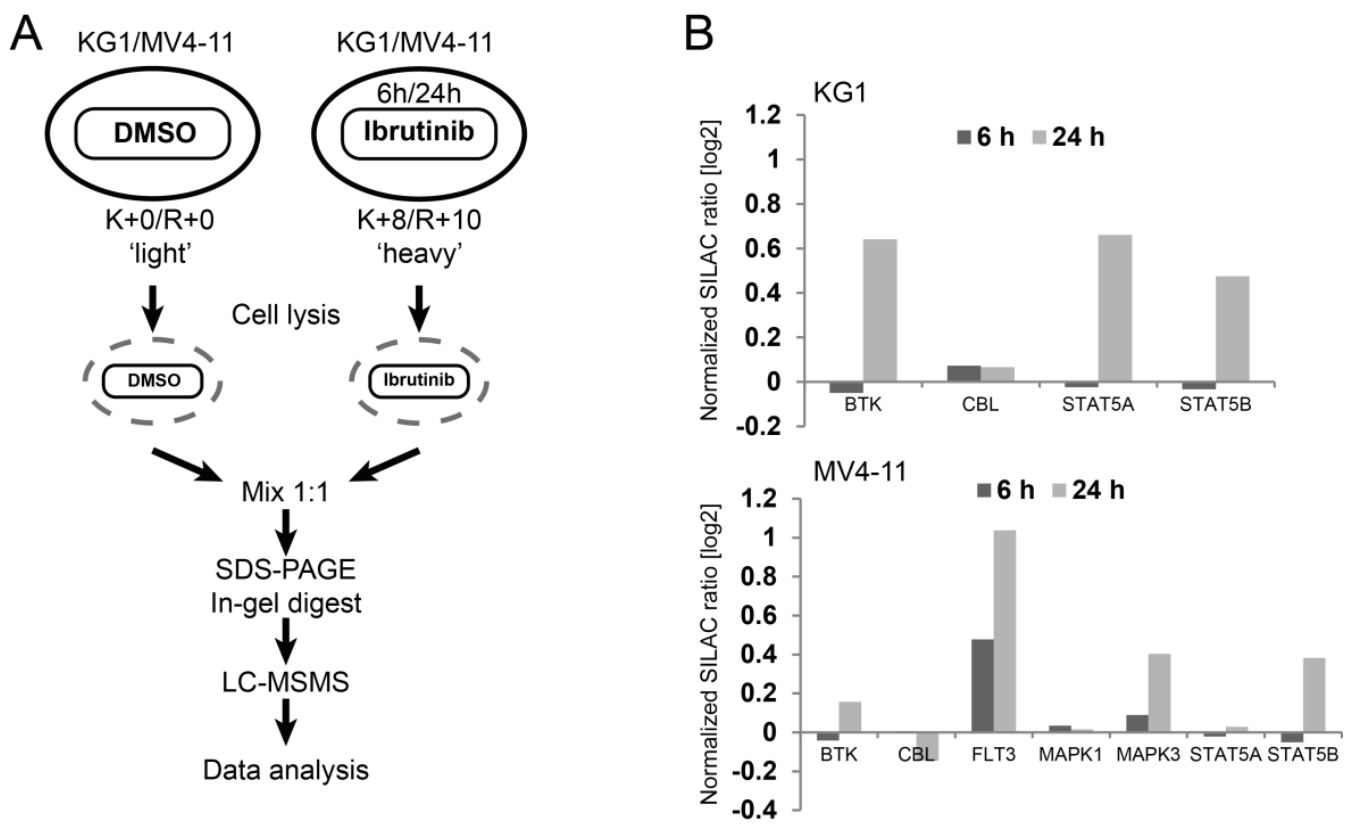

Figure 3.20: Protein expression changes after BTK inhibition in AML

(A) Experimental design of the SILAC-based protein expression analysis in KG1 and MV4-11 cells after BTK inhibition. Heavylabeled cells were treated for $6 \mathrm{~h}$ or $24 \mathrm{~h}$ with ibrutinib, light-labeled cells were treated with DMSO as a control. Proteins were analyzed by mass spectrometry after in-gel digestion. (B) Bar diagrams displaying the normalized SILAC ratios H/L (log2) for selected proteins identified as relevant from the phosphotyrosine analysis (BTK, FLT3, MAPK1/3, STAT5A/B) for KG1 and/or MV4-11 after $6 \mathrm{~h}$ and $24 \mathrm{~h}$ of ibrutinib treatment. Cell lines were cultured and treated as shown in (A) at the Department of Internal Medicine, Hematology/Oncology at the University Hospital Frankfurt/Main as part of the collaboration mentioned in Table 12. Protein expression values are listed in supplementary table S12. 


\subsection{Phosphorylation dynamics in TCR signaling adapters}

The antigen recognition process of the TCR leads to complex downstream signaling cascades which are as antigen-dependent signal initiation in B cells mainly mediated by reversible phosphorylation of kinases and adapter proteins such as SLP76 (LCP2), GADS (GRAP2), GRB2, LAT, ZAP70, LCK and many others. TCR signaling responses depend on a heterotrimeric complex consisting of three adapter proteins: SLP76, GADS and LAT (Chakraborty and Weiss, 2014; Kambayashi et al., 2009; Koretzky et al., 2006; Lugassy et al., 2015). Large-scale MSbased $p$-sites analyses in TCR signaling have been previously performed, however, phosphorylation of the adapter proteins SLP76 and GADS were not covered in these datasets (Cao et al., 2012; Nguyen et al., 2009). The phosphorylation changes of SLP76 and GADS signaling adapters were studied in the SLP76-deficient Jurkat derivative cell line J14 reconstituted with a STREP-tagged SLP76 (Yablonski et al., 1998a). The Jurkat T-cell line is an established model to study TCR signaling with a long history of major scientific breakthroughs made in the field of signaling e.g. the detection of intracellular $\mathrm{Ca}^{2+}$ flux, tyrosine phosphorylation after TCR stimulation and the role of ITAM motifs in signal transmission (Abraham and Weiss, 2004). For the comparison of the ground to the TCR stimulated state, cells were duplex SILAC labeled: the TCRs of the heavy-labeled cells were stimulated for 1, 2, 5 and 10 min respectively whereas the light-labeled cells remained in the non-stimulated state. Cell lysates from equal cell numbers of light- and heavy-labeled cells were mixed and the STREP-tagged SLP76 was affinity purified. Proteins were separated on a SDS-PAGE gel, SLP76 and GADS were excised from the gel and digested with three endoproteinases, respectively in order to increase protein sequence coverage as described previously in section 2.2.2.2.3. Phosphopeptides were enriched with $\mathrm{TiO}_{2}$ affinity purification from the in-gel digests prior to LC-MS/MS analysis (Figure 3.21 A).

\subsubsection{SLP76/LCP2 phosphorylation site dynamics}

SLP76 is expressed in all cells derived from the hematopoietic lineage except for B cells where a similar protein, SLP65 (or BLNK) is expressed (Fu et al., 1998; Wienands et al., 1998). SLP76 contains an $\mathrm{N}$-terminal acidic region with a SAM domain, a central proline-rich domain which mediates interaction with GADS and a C-terminal SH2 domain (Myung et al., 2001). For SLP76, 33 non-redundant class I p-sites could be identified ( 25 pS, 4 pT and 4 pY) within this study. 30 p-sites (22 pS, 4 pT and 4 pY) could be quantified in the singly and multiply phosphorylated state in at least one biological replicate and one stimulation time point, respectively (data not 
shown). In order to deduce differences in phosphorylation behaviour, p-sites were required to be quantified in at least three of four biological replicates quantified over the whole time course were considered for further analysis. This resulted in $11 \mathrm{p}$-sites (9 pS, 1 pT, 1 pY) which fall in separate categories: 'steady-state', 'late' and 'early' inducible (Figure 3.21 B). In general, SLP76 phosphorylation sites are functionally not well studied. The analysis presented here provides a reference point for further functional studies. However, sites that have been functionally studied and described will be discussed and set in context to the phosphorylation dynamics identified in this experimental work.

A

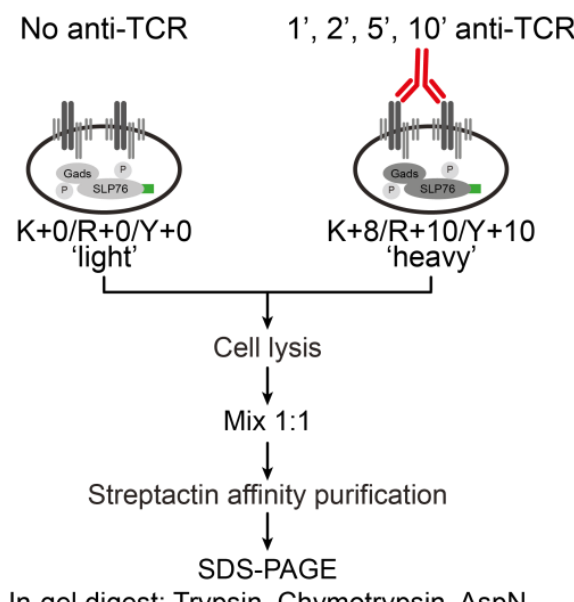

In-gel digest: Trypsin, Chymotrypsin, AspN

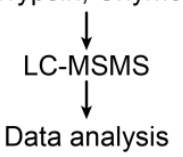

B
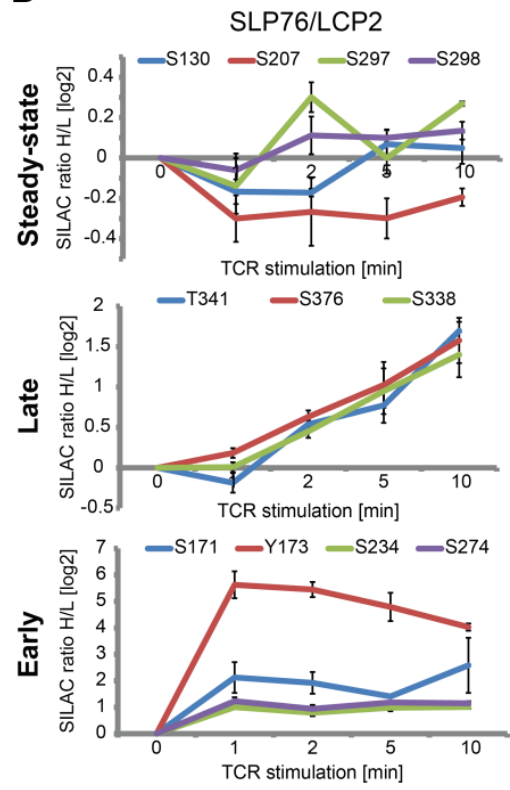

C
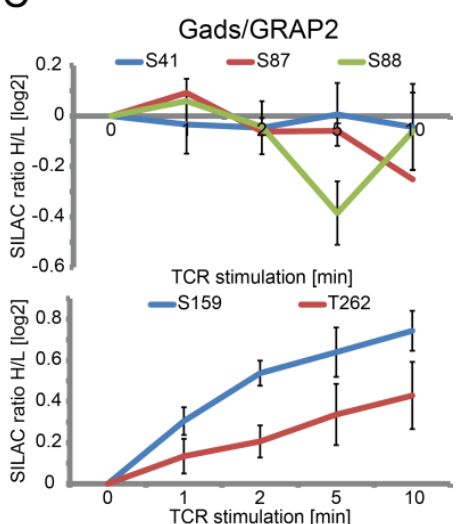
西 
All SLP76 p-sites quantified according to the above mentioned conditions are located between the N-terminal SAM and the C-terminal SH2 domain. SLP76 S376 was identified as a 'late' phosphorylation site and is known to be associated with SLP76 ubiquitination followed by its degradation (Figure 3.21 B, middle panel, red line) (Wang et al., 2012). Therefore, SLP76 S376 'late' phosphorylation could be a part of a negative feedback loop leading to signal determination by adapter protein degradation. As putative upstream kinase MAP4K HPK1 was identified (Di Bartolo et al., 2007; Wang et al., 2012). The two p-sites SLP76 S338 and T341 showed the same phosphorylation behavior as S376 but have not been functionally associated. The phosphotyrosine site SLP76 Y173 was of particular interest as it was functionally studied in earlier collaboration experiments with the group of Debbie Yablonski (Sela et al., 2011). The p-site Y173 showed an 'early' increase in phosphorylation already after $1 \mathrm{~min}$ of TCR stimulation which slightly decreased until $10 \mathrm{~min}$ but maintained at an overall high level (Figure $3.21 \mathrm{~B}$, lower panel, red line). In the previous study, a Tec family kinase was identified as the SLP76 Y173 modifying kinase. Furthermore, phosphorylated Y173 was identified to be a prerequisite for the phosphorylation-induced activation of the phospholipase C- $\gamma 1$ (PLC- $\gamma 1$ ) (Sela et al., 2011). The recruitment of the Tec family kinase to SLP76 depends on three ZAP70-targeted tyrosines at the N-terminus of SLP76 which were not identified in the analysis presented here. Recently, SLP76 Y173 became of particular interest as the activation mechanism was revealed by Devkota et al., 2015. Phosphorylated Y173 provides a binding site for the C-terminal SH2 domain of PLC- $\gamma 1 \mathrm{C}$. After binding, PLC- $\gamma 1 \mathrm{C}$ gets phosphorylated by the SLP76-associated Tec family kinase at Y738 and therefore becomes enzymatically active (Devkota et al., 2015).

\subsubsection{GADS/GRAP2 phosphorylation site dynamics}

GADS (also known as GRAP2 or p38) belongs to the Grb2 family of adaptor proteins. It possesses an $\mathrm{N}$ - and $\mathrm{C}$-terminal $\mathrm{SH} 3$ and a central $\mathrm{SH} 2$ domain and resides in the cytoplasm. The C-terminal SH3 domain binds constitutively to the cytoplasmic adaptor SLP76 and becomes therefore co-purified with SLP76. 14 p-sites (10 pS, 2 pT and 2 pY) on GADS were identified as class I p-sites presented in this work from which 13 sites were identified in at least one biological replicate in at least one time point of stimulation (data not shown). Five p-sites were quantified in at least three of four biological replicates with a complete phosphorylation profile (Figure $3.21 \mathrm{C}$ ). The five sites (4 pS, 1 pT) could be divided in two 
distinct categories: 'steady-state' and 'late' inducible, missing the 'early' inducible which can be seen for SLP76 (Figure 3.21 C, upper and middle panel). GADS S41, S87 and S88 showed no major regulation behavior after TCR stimulation and were classified as 'steady-state' p-sites (Figure $3.21 \mathrm{C}$, upper panel). S41 lies in the N-terminal SH3 domain, whereas S87 and S88 are positioned in the central SH2 domain. GADS S159 and T262 showed a 'late' regulation behavior (Figure $3.21 \mathrm{C}$, middle panel). Both $\mathrm{p}$-sites lie within the linker region between the SH2 and the C-terminal SH3 domain. T262 is proposed to be a phosphorylation site involved in tonic TCR signaling with a slight phosphorylation response after TCR stimulation (Figure 3.21 C, middle panel, red line) (Lugassy et al., 2015). The orthologous murine p-site to human T262 (mouse: T254) is proposed to be a negative regulatory $p$-site that may promote the detachment of GADS from the LAT-nucleated signaling complex (Lasserre et al., 2011). However, the functional mechanism is not known and needs to be identified. 


\section{Discussion}

The presented work focused on the global investigation of phosphorylation sites in diverse experimental conditions by mass spectrometry-based phosphoproteomics. As the main focus was set on Burkitt's lymphoma, the results of this main project will be discussed in the first part in terms of comparability to other large-scale PTM studies, sample preparation including PTM enrichment, LC-MS/MS instrumentation, and the integration of other large-scale datasets with phosphoproteomic data. In the second part, the biological impact of this work will be discussed such as the BCR signaling signature in different Burkitt's lymphoma genotypes. Additionally, the tonic signaling models and single proteins that appeared to be interesting candidates for further investigations or might present drug targets will be highlighted.

\subsection{Mass spectrometry-based large-scale studies}

Mass spectrometry is a suitable tool for the hypothesis-free, large-scale analysis of proteins and PTMs. The up to now most comprehensive human proteome draft was presented in 2014 by the groups of Akhilesh Pandey and Bernhard Küster, providing evidence for more than $80 \%$ of the human protein-coding genes on the protein level by mass spectrometry (Kim et al., 2014; Wilhelm et al., 2014). However, merely the identification, relative quantitation or even the copy number of proteins does not necessarily provide evidence about their activity, interaction capabilities or subcellular localization in a given cellular context (reviewed in Larsen et al., 2006). Therefore, one ultimate aim of understanding the diversification of cellular behavior is the identification, quantitation, and functional characterization of all PTMs in a certain state of a cell at a given time point.

\subsubsection{Phosphorylation in cell signaling}

The rise of large-scale, mass spectrometry-based PTM studies began with the identification of a few hundred phosphorylation sites (Ficarro et al., 2002). Numbers have ever since increased: In 2004, a HeLa nuclear phosphoproteome consisting of 2,002 p-sites was published (Beausoleil et al., 2004). In 2006, 6,600 phosphorylation sites were identified in HeLa cells after EGF stimulation (Olsen et al., 2006). In 2009, a study was published that identified 10,844 psites in human embryonic stem cells (Swaney et al., 2009). In 2010, 20,443 p-sites were 
identified during cell cycle progression in HeLa cells (Olsen et al., 2010). The largest number of identified p-sites to date was published in 2014 , when more than 38,000 class I p-sites were identified in EGF stimulated HeLa cells (Sharma et al., 2014). The workflow for phosphopeptide enrichment in this study consisted of FASP-based proteolysis, SCX peptide fractionation, and $\mathrm{TiO}_{2}$ affinity purification for global phosphoproteomic analysis. Specific enrichment of phosphotyrosine residues was done with antibodies directed against phosphotyrosine residues (Sharma et al., 2014). Samples were measured on a hybrid quadrupole-Orbitrap mass spectrometer and quantified by XIC-based label-free quantitation (Sharma et al., 2014).

Within this study presented here, approximately 4,700 p-sites were identified after BCR stimulation in DG75 cells (supplementary tables S2 and S3). Triplex SILAC labeling hinders sequencing depth due to increased complexity on the MS1 level. This can be circumvented by using label-free quantitation as demonstrated by Sharma et al., 2014. Furthermore, the use of faster, more sensitive MS instruments contributes to higher identification rates. The large number of p-sites shown by Sharma et al., 2014 are impressive, however, cells were treated with pervanadate, a tyrosine phosphatase inhibitor, which leads to accumulation of $p$-sites and does not necessarily reflect the physiological cellular state (Sharma et al., 2014; Zhao et al., 1996).

\subsubsection{Acetylation and ubiquitination in cell signaling}

The development of modification-specific antibodies against acetylated and di-glycine modified lysine residues made large-scale MS-based acetylation and ubiquitination studies feasible (Choudhary et al., 2009; Kim et al., 2006; Xu et al., 2010). One of the first global lysine acetylation studies identified 3,600 acetyl-lysine sites (Choudhary et al., 2009). A large-scale ubiquitination study lead to the identification of 11,054 di-glycine modified lysine residues in HEK293T and MV4-11 cells (Wagner et al., 2011). The characterization of acetylation and ubiquitination in BCR signaling in Burkitt's lymphoma shown in this work (section 3.1.11) did not reach the analysis depth of the studies mentioned above. The analyses in this work should rather be considered as preliminary feasibility study. Further analyses require sample preparation refinements such as the titration of sample to antibody ratio, the use of proteasome, DUB and KDAC inhibitors in the lysis buffers, respectively. Furthermore, the alkylating agent needs to be replaced by chloroacetamide in the ubiquitination study as IAA was reported to mimic the di-glycine adduct resulting from protein hydrolysis with trypsin (Nielsen et al., 2008). In general, results obtained from the ubiquitination study are difficult to 
put into a biological context as after proteolytic digest with trypsin, mono- and the differently linked poly-ubiquitination chains cannot be distinguished anymore. Therefore it is not possible to differentiate between ubiquitination-mediated signaling and tagging for degradation.

\subsubsection{Large-scale B-cell receptor signaling studies}

T-cell signaling has been extensively studied in low- and high-throughput studies in the past whereas B cells remained underrepresented. Early mass spectrometric studies investigating phosphorylation-mediated B-cell signaling in DT40 (chicken) were low-throughput studies based on single proteins such as SLP65, SYK and GRB2 (Bohnenberger et al., 2011; Neumann et al., 2009; Oellerich et al., 2011; 2009). As the next step to understand B-cell signaling, a global analysis of phosphorylation-mediated signaling processes in human B cells was performed. The investigation of antigen-dependent and -independent signaling in Burkitt's lymphoma derived B cells on the phosphoproteomic level presented within this work is conceptually novel. However, the investigation focus of other groups has recently moved to $B$ cells and large-scale, MS-based phosphoproteomic signaling studies of activated antigendependent BCR signaling in different B-cell types with similar methodology (Rolland et al., 2014; Satpathy et al., 2015; Tsai et al., 2014). Some of the studies will be discussed in terms of number of identified $\mathrm{p}$-sites, methodology and interesting proteins with regulated $\mathrm{p}$-sites. None of the previously mentioned studies were performed on DG75 cells, however two phosphoproteomic studies based on DG75 emphasizing methodical aspects of phosphopeptide enrichment rather than giving biological insights will not be discussed in the following (Iliuk et al., 2010; Pan et al., 2015).

Rolland et al., 2014 investigated differences in the global phosphoproteomes in Burkitt's lymphoma, follicular lymphoma (both germinal center and antigen-experienced cells) and mantle cell lymphoma (naïve B cells) cell lines. The BCRs of the cells were not stimulated, reflecting the general tonic signaling state that might be affected by growth factors in the culture medium and is thus not considered as being representative for tonic BCR signaling (Rolland et al., 2014). Differentiation of the different lymphoma subtypes was achieved by spectral counting. $\mathrm{TiO}_{2}$ and antibody-based phosphotyrosine enrichment lead to the identification of 6,579 p-sites (Rolland et al., 2014). Rolland et al., 2014 found the involvement of proximal BCR signaling proteins as a hallmark of BL and follicular lymphoma compared to naïve $B$ cells. This is also reflected in the study presented here as many regulated effector proteins in any condition are known B-cell effectors (Figure 3.6; section 3.1.13; and 
supplementary table S10) (Rolland et al., 2014). Furthermore, Rolland et al., 2014 showed an increase of phosphorylation of several tyrosine residues of the lipid-raft membrane anchored PAG1 protein in germinal center-derived Burkitt's lymphoma cell lines compared to naïve B cells (Rolland et al., 2014). Most of the described tyrosine p-sites of PAG1 were quantified as upregulated as well in the BCR stimulation time course of DG75, Daudi and FFM_BL1 Burkitt's lymphoma cells, and in the tonic signaling conditions in DG75 presented in this study (supplementary tables S3, S9, and S10). Differences can be attributed to the use of different Burkitt's lymphoma cell lines which confer genotype specificity especially in BCR distal phosphorylation sites as described in section 3.1.13. Quantitation was achieved by spectral counting which might be sufficient for rough estimates of protein expression levels, but is not reliable enough for the quantitation of low abundant species such as phosphopeptides (section 1.3.6) (Cox et al., 2014) (reviewed in Bantscheff et al., 2007; 2012). While writing this thesis, a study was published which resembles the work presented here closely and therefore will be discussed in more detail (Satpathy et al., 2015). The authors investigated BCR signaling in a murine B-cell lymphoma cell line, expressing a BCR of the IgG isotype. Signaling was monitored after 5 and $15 \mathrm{~min}$ of $\mathrm{BCR}$ stimulation on the phosphoproteomic and the ubiquitination level; tonic BCR signaling was not analyzed (Satpathy et al., 2015). Quantitation was achieved by triplex SILAC labeling, and PTMs were enriched in a dual approach by either combining $\mathrm{TiO}_{2} / \mathrm{SCX}$ for phosphopeptide enrichment or by antibody-based phosphotyrosine enrichment (Satpathy et al., 2015). The phosphopeptide enrichment approaches lead to the identification of 10,663 class I p-sites. Compared to the approximately 4,700 p-sites quantified within this study (supplementary tables S2 and S3) Satpathy et al., 2015 identified significantly more $p$-sites. This can be partially attributed to performing measurements on a $Q$ Exactive instrument for the reasons mentioned previously in section 4.1.1. Furthermore, the BCR stimulation time course with double triplex SILAC labeling to cover four time points in DG75 cells shown in this study is more complex. For full quantitation across the whole time course more stringent criteria need to be applied which leads to a decrease in overall sites. $29.3 \%$ $(3,121)$ of the $p$-sites identified by Satpathy et al., 2015 were considered to be regulated after 5 and/or $15 \mathrm{~min}$ with a SILAC ratio fold-change $\geq 2$ or $\leq 0.5$ (corresponding to $\leq-1$ or $\geq 1$ on a $\log 2$ scale). Although the regulation threshold for the global phosphoproteome presented in this work was lower (0.75-fold on a log2 scale), overall only $19.2 \%$ of the quantified $p$-sites over the BCR stimulation time course in DG75 were considered as regulated (supplementary 
table S2 and S3). Apart from the methodological differences mentioned above, B cells used for both studies differed in the isotype of their BCR: in this work, cells with a BCR of the IgM isotype were used, whereas Satpathy et al., 2015 used cells with a BCR of the IgG isotype. Receptors of the IgM isotype have different signaling outcomes compared to that of the IgG isotype due to the different constant region in the cytoplasmic tails (Silver and Cornall, 2003; Wakabayashi et al., 2002). Notably, the cytoplasmic tail of IgG BCRs harbors a signaling motif that is considered as an intrinsic enhancer of BCR signaling (Engels et al., 2009). Both projects share the same basic approach, however they differ not only with regard to genotype and Ig isotype, but also to species. The datasets were therefore mapped with the online tool PhosphOrtholog that matches p-sites across different species (www.phosphortholog.com; Chaudhuri et al., 2015). Comparison of 10,663 quantified class I p-sites derived from mouse B cells to 4,176 unique, quantified class I p-sites derived from human DG75 B cells revealed 1,109 common p-sites (Figure 4.1 A). For the determination of similarity of both datasets, the SILAC ratios after $5 \mathrm{~min}$ of $\mathrm{BCR}$ stimulation respectively were plotted against each other (Figure $4.1 \mathrm{~B}$ ). Overall, correlation of both datasets is moderate, while commonly regulated psites are derived from SYK, CD79A, PRKCD and other formerly known proximal B cell effectors (Figure 4.1 B).

A

Satpathy et al. (2015)

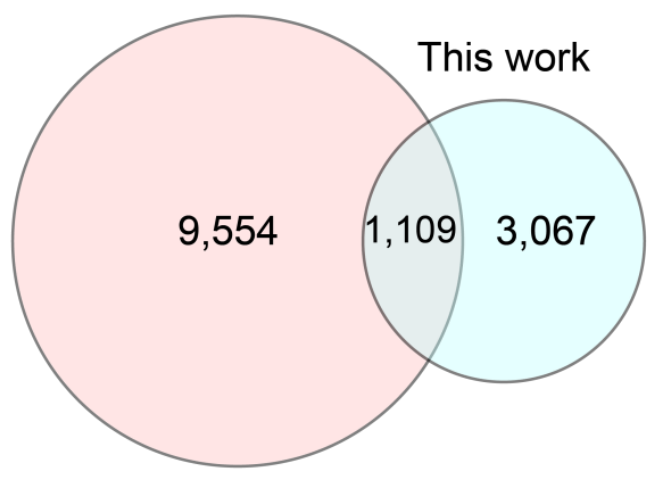

B

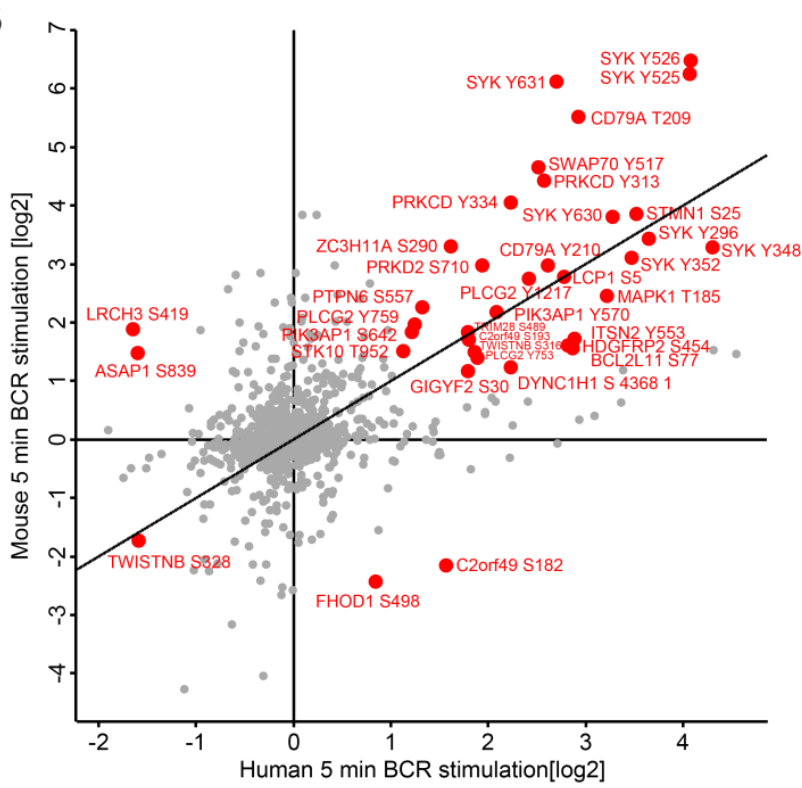

Figure 4.1: Comparison of $p$-sites after 5 min of BCR stimulation in mouse and human B cells

All quantified p-sites from both datasets were submitted to the online tool PhosphOrtholog (www.phosphortholog.com; Chaudhuri et al., 2015). (A) 1,109 p-sites overlapped between the Satpathy et al., 2015 dataset with 10,663 quantified mouse $\mathrm{p}$-sites and the dataset with 4,176 unique, quantified human $\mathrm{p}$-sites shown in this work. (B) Common $\mathrm{p}$-site ratios after 5 min of BCR stimulation from both datasets were plotted against each other. The $x$-axis shows normalized SILAC ratios of human $B$ cells and the $y$-axis the normalized SILAC ratios of mouse B cells after 5 min of BCR stimulation, respectively. 
Satpathy et al., 2015 as well as Tsai et al., 2014 performed their experiments in mouse and not in human B-cell models. It needs to be further elucidated whether and how the results derived from other species can be related to human B-cell physiology (Satpathy et al., 2015; Tsai et al., 2014). The phosphoproteomic BCR stimulation time course presented in this study and by Satpathy et al., 2015 were analyzed within the minute range. In another study performed on antigen-dependent TCR signaling, the time-resolved phosphoproteome was analyzed after 5 to $60 \mathrm{~s}$ of receptor stimulation. Changes could be already identified after $5 \mathrm{~s}$ of TCR stimulation suggesting that phosphorylation-mediated signaling occurs almost immediately after the stimulus (Chylek et al., 2014). Due to the similarities of TCR to BCR signaling (described in section 1.1) antigen-dependent signaling in B cells most likely also occurs within a shorter time window and should be considered in designing time-resolved receptor stimulation experiments in future.

\subsection{Sample preparation and LC-MS/MS instrumentation}

As mentioned earlier in section 4.1.1 and 4.1.3, the number of identified and quantified psites has steadily increased over time. The number of phosphorylation sites - or in general PTM sites - that can be quantified depends mainly on the enrichment method and LC-MS/MS instrumentation. Therefore the development, evaluation and improvement of state-of-theart enrichment methods has been a popular topic in the mass spectrometric community in recent years. At the beginning of the project, $\mathrm{SCX}$ fractionation prior to $\mathrm{TiO}_{2}$ spin column enrichment with DHB as selective competitor was considered as the standard enrichment protocol for phosphopeptides (Thingholm et al., 2007; Zarei et al., 2011). Scaling of the peptide-to-bead ratio influences the selectivity of phosphopeptide enrichment. Fractionation entails difficulties in determining the final peptide amount for each fraction and accordingly scaling of $\mathrm{TiO}_{2}$ bead material ( $\mathrm{Li}$ et al., 2009). This has been addressed by swapping the fractionation and the enrichment step, which has the additional advantage that $\mathrm{TiO}_{2}$ enrichment can be performed on a batch basis instead of enriching each SCX fractions separately. Furthermore, DHB was replaced as competitor by more LC-MS/MS compatible and selective alternatives such as TFA, glycerol or glutamic acid (Fukuda et al., 2013; Thingholm et al., 2007). Basic reversed-phase (BRP) fractionation proved to be a good alternative to SCX fractionation as separation of peptides occurs according to hydrophobic interactions instead of the peptide charge state (Batth et al., 2014). Therefore, a higher separation resolution can 
be reached, leading to a higher depth of mass spectrometry measurement (Batth et al., 2014). BRP is also suitable for other global PTM studies besides phosphorylation (Batth et al., 2014; Fukuda et al., 2013; Li et al., 2009; Mertins et al., 2013). Apart from methodological refinements, developments in LC-MS/MS instrumentation also contribute to an increasing number of PTM identifications. Ultra-high performance liquid chromatography systems allow a more reproducible separation and therefore more sophisticated algorithms for following data analysis such as the 'match between runs' option. This option transfers MS/MS identifications from one LC-MS/MS run to others where the peptide is not substantiated by a product ion spectrum but only its exact mass and the retention time (Geiger et al., 2010). Furthermore, newer mass spectrometers such as hybrid quadrupole-Orbitrap instruments perform shorter duty cycles and fragment spectra analyzed in the Orbitrap have a higher resolution and accuracy compared to the previously used hybrid linear ion trap-Orbitrap instruments (Michalski et al., 2011; Olsen et al., 2009; Sun et al., 2012). This leads to higher identification rates and improved PTM localization.

The global phosphoproteome of the DG75 BCR stimulation time course as presented in this work was enriched by the combination of $\mathrm{SCX}$ and $\mathrm{TiO}_{2}$ affinity purification and measured on the LTQ Velos with ion trap CID fragmentation as described previously (2.2.3.1). After applying the filtering criteria mentioned in section 2.2.5.1, approximately 4,400 p-sites were quantified (Figure 3.3; supplementary tables S2). For the scope of the project, the global phosphoproteome analysis of BCR stimulated Daudi cells was enriched with $\mathrm{TiO}_{2}$ affinity purification as a first step followed by BRP chromatography, and was measured on a $Q$ Exactive HF instrument (data not included in this work). The global phosphoproteome analysis of Daudi cells quantified approximately 7,100 class I p-sites over the time course of BCR stimulation. However, it needs to be considered that the Daudi time course does not contain four, but only two time points. Furthermore, more stringent filtering criteria concerning the quantitation across biological replicates in the DG75 dataset were applied and therefore might underestimate the number of $\mathrm{p}$-sites that could be quantified. Nevertheless, $\mathrm{TiO}_{2} / \mathrm{BRP}$ is compared to $\mathrm{SCX} / \mathrm{TiO}_{2}$ more robust, requires less input material and can be also applied with adaptations to label-free approaches. 


\subsection{Determination of transcription factor activation}

The Burkitt's lymphoma phosphoproteomic analysis was supplemented with complementary analyses for example RNA seq analysis to monitor BCR stimulation-induced transcriptional changes and activation of transcription factors (TF). In the study presented here, activation of transcription factors was derived from RNA seq analysis by mapping overexpressed transcripts to database-stored transcription factors and performing an enrichment analysis as described previously (2.2.6.1.2). Furthermore, quantified $p$-sites of the particular TFs were mapped and manually evaluated for their potential influence on the activation status by information stored in the UniProt and PSP database (3.1.7). In general, transcriptional regulation is a complex process that requires for example the presence of activating histone marks, the binding of TFs, their activation, and the recruitment of RNA polymerase II (Simon and Kingston, 2013). Histone marks can be investigated by mass spectrometry or western blot analysis (reviewed in Strahl and Allis, 2000; Villar-Garea and Imhof, 2006). TF-DNA interactions can be globally determined by chromatin immunoprecipitation (ChIP seq) (Johnson et al., 2007; Park, 2009; Valouev et al., 2009; Whitfield et al., 2012). Like kinases, TFs possess phosphorylation sites indicating activation which can be investigated by immunoprecipitation or western blot (Hunter and Karin, 1992; Whitmarsh and Davis, 2000). Furthermore, RNA polymerase II recruitment can be investigated using anti-RNA polymerase II antibodies in ChIP analysis (Johnson et al., 2001). However, all mentioned approaches alone do not necessarily reflect the activity of a particular TF, therefore a combination of complementary approaches and computational modelling is necessary to predict and deduce activation of TFs (Gao et al., 2004) (reviewed in Banerjee and Zhang, 2002). An advantage of the approach presented here is the combination of two global high-throughput approaches, namely RNA seq and phosphoproteomic analysis. Drawbacks of the MS analysis might be posed by stochastic identification of TFs, and transcript-TF mapping relies on the quality of the database. The approach for the determination of activated transcription factors presented in this work could present an additional strategy to the tools mentioned previously. However, a few methodological refinements should be implemented such as a higher congruence of the time scale of the phosphoproteomic and the RNAseq analysis time courses. The global phosphoproteomic time course covered the BCR-induced stimulation after 2, 5, 10 and 20 min, whereas the RNA seq analysis was performed after $10 \mathrm{~min}, 20 \mathrm{~min}, 1 \mathrm{~h}$, and $2 \mathrm{~h}$ after BCR stimulation. Although it can be assumed that transcriptional regulation lags phosphorylation 
changes, very fast transcriptional changes were observed for example for FOS, HSPA6, HSPA7, EGR1, and EGR3 already after 10 or 20 min of BCR stimulation (section 3.1.7; supplementary table S4). Therefore, transcriptional regulation should be monitored in a shorter time window as done within this work.

\subsection{Genotype-specific and tonic BCR signaling in BL}

The main project for this work focused on the systematic investigation of signaling mechanisms in the Burkitt's lymphoma cell line DG75. Uniform, activated upstream signaling events in DG75, Daudi and FFM_BL1 Burkitt's lymphoma cell lines could be found on early upregulated 'classic' BCR signaling proteins (supplementary tables S2, S3, and S9). Also differential phosphorylation patterns of tyrosine residues on BCR signaling proteins could be detected for example on BTK and CD37 (3.1.13). Heterogeneity between the different cell lines can be explained by the different clinical subtypes: DG75 and FFM_BL1 are derived from sporadic BL disease whereas Daudi is an endemic, EBV-positive BL. DG75 and Daudi have a MYC translocation in common whereas FFM_BL1 possesses only MYC point mutations. Besides the biological aspect, FFM_BL1 p-sites were quantified with a label-free approach introducing additional methodical variation, as quantitation might not be as accurate as SILAC labeling. However, the differences in signaling outcomes are surprising as distinct diseased cells are indistinguishable from the histological and clinical point of view. Comparative analysis of the global phosphoproteomes of DG75, Daudi and FFM_BL1 covering mainly phosphoserine and -threonine residues showed an even higher variation in signaling responses and posed a challenge for analysis of these datasets (data not included in this work).

Antigen-induced BCR signaling was intensively studied in different experimental conditions $(3.1 .3,3.1 .7,3.1 .8,3.1 .9,3.1 .10,3.1 .11$, and 3.1.13). Burkitt's lymphoma is thought to be mainly driven by tonic signaling that is dependent on a functional BCR but independent of antigen binding (Schmitz et al., 2012; Srinivasan et al., 2009). Tonic BCR signaling was investigated in two models by phosphotyrosine enrichment: an inducible knockdown of the ITAM-containing BCR signaling chain CD79A and pharmacological SYK ablation (3.1.14). For the scope of this work, the tonic BCR signaling analysis was planned to be performed in Daudi cells as well. After CD79A knockdown, Daudi cells could not be cultured to achieve sufficient cell numbers required for phosphoproteome analyses (data not shown). CD79A knockdown performed in several $B L$ and $A B C-D L B C L$ cell lines showed that it leads to a reduced $B C R$ 
expression on the cell surface and eventually to reduced cell viability of Daudi cells after several days (Schmitz et al., 2012). Therefore, tonic BCR signaling could only be analyzed in DG75 cells. Hampered cell growth and viability in cell culture was also observed in DG75, however to a lesser extent. The different responses to the CD79A knockdown furthermore point towards genotype specificity of signaling cascades as it can be tolerated by one cell line but not the other. Both tonic signaling conditions were designed as complementary models as SYK activation is dependent on binding to the tandem phosphorylated ITAM motif in CD79A (Figure 1.1). Therefore, it was expected that signal inhibition from either of the two directly interacting signaling molecules leads to the same or at least a similar cellular signaling outcome. Surprisingly, the data presented in this work showed that the correlation of identified $\mathrm{p}$-sites derived from the pYome of both tonic signaling models is minor (Figure 3.15 C). Although commonly regulated $p$-sites on MAPK1 and MAPK3 were identified, the majority of p-sites were differently regulated (Figure 3.15 C; supplementary table S10). Partial results render data interpretation even more challenging such as the lack of detection of autophosphorylation and activating SYK p-sites after BCR knockdown and Syk inhibition or in general a low number of SYK target p-sites (supplementary table S10). The results could hint towards SYK independency of tonic signaling although, SYK dependence was described in diffuse large B-cell lymphoma (DLBCL) a disease similar to BL (Chen et al., 2007). However, DLBCL has point mutations in the ITAM motif of CD79A and B which induce chronic activated BCR signaling and which are not present in Burkitt's lymphoma (Davis et al., 2010; Schmitz et al., 2012). The SYK inhibitor PRT062607 has been reported to be selective. Off-target effects on other kinases could still lead to a different signaling outcome (Spurgeon et al., 2012). It is known that tonic signaling not only depends on the BCR but also on other receptors such as the B cell-activating factor receptor (BAFF-R) (Rowland et al., 2010; Srinivasan et al., 2009; Stadanlick et al., 2008). From the data presented here, tonic signaling could also depend on other receptors such as CD37 as described in section 4.5.4.

Tonic signaling is thought to involve fewer pathways compared to antigen-dependent signaling and is mainly driven via the PI3K pathway (Srinivasan et al., 2009; Young and Staudt, 2013a). Schmitz et al., 2012 followed PI3K pathway activity after CD79A knockdown by FACSbased analysis of the phosphorylation status of AKT S473 in different BL cell lines including Daudi. After the knockdown, reduced phosphorylation of AKT S473 could be observed in all BL cell lines, thereby providing evidence for PI3K involvement in BL (Schmitz et al., 2012). The 
site could not be quantified in any of the BL cell lines or experimental conditions shown in this work. In general, many $\mathrm{p}$-sites involved in PI3K signaling that were found regulated upon antigen-dependent BCR signaling but could not be detected or were not regulated in tonic $\mathrm{BCR}$ signaling in DG75, render data interpretation challenging (supplementary table S10).

\subsection{Drug targets in Burkitt's lymphoma}

As targeted therapeutic strategies for Burkitt's lymphoma are lacking it is commonly treated by multiagent chemotherapy which is a highly aggressive treatment regimen (Blum et al., 2004). Therefore, one aim of this study was to identify potential effector proteins that are suitable for targeted pharmacological inhibition. In the following, proteins that appeared as interesting candidates within this study will be discussed with regard to their functional role in BL signaling and/or therapeutic suitability.

\subsubsection{BRAF}

The MAP-kinase BRAF was already introduced as a potential therapeutic target based on dephosphorylation of the putative inhibiting p-site S363 in combination with enhanced affinity to kinobeads indicating its activation (section 3.1.8, Figure 3.8 and Figure 3.9). BRAF is known to be involved in different cancers, for example melanoma. In melanoma, BRAF is commonly mutated with a single amino acid substitution in the kinase domain (V600E). The mutation renders the kinase constitutively active, thus making it independent of RAS activation, and as a consequence drives the RAF-MEK-ERK signaling axis (Brose et al., 2002; Cheung et al., 2008; Davies et al., 2002a; Hatzivassiliou et al., 2010). Specific inhibitors targeting mutated BRAF are available and have proven to be a valuable treatment in melanoma (Flaherty et al., 2010b). For the inhibition of BRAF in DG75 cells as shown in section 3.1.10, the V600E BRAF inhibitor Raf265 was used (Williams et al., 2015). However, mutated BRAF has not been associated with Burkitt's lymphoma (Love et al., 2012; Schmitz et al., 2012). Application of BRAF inhibitor in non-mutated BRAF can lead to its paradoxical activation by drug-mediated transactivation (Hatzivassiliou et al., 2010; Poulikakos et al., 2010). From the global phosphoproteome dataset shown in 3.1.10, it cannot be concluded if BRAF was inhibited or activated as the activating $p$ sites of MAPK1 and MAPK3 were found to be slightly upregulated after BCR stimulation in combination with BRAF inhibition compared to BCR stimulation only (supplementary table S7). To determine kinase activity based on a single $p$-site might not provide enough analysis depth as BRAF possesses further activating and inhibiting $p$-sites that were not detected (Chong et 
al., 2001). Furthermore, it is not clear if BRAF activation can be generalized to all BL models as BRAF S363 was not quantified in the global phosphoproteomes of BCR stimulated Daudi and FFM_BL1 BCR (data not shown) and signaling dynamics seem to highly depend on genetic background as stated previously in section 4.4. Taken together, BRAF is an interesting antigendependent $B C R$ signaling effector identified in $B L$ within this study. However, the paradoxical activation and the missing evidence of activation in BL cells with a distinct genotype make BRAF as target for pharmacological inhibition presumably unsuitable.

\subsubsection{HSP90}

Another interesting and possibly druggable target identified within this work was the heatshock protein 90 (HSP90). One phosphotyrosine site on HSP90AA1 was found upregulated in the BCR stimulation time course in DG75 cells (supplementary table S3). Another phosphotyrosine site was identified as upregulated on HSP90AB1 in the BCR stimulation time courses in DG75 cells as well as in Daudi and FFM_BL1 cells (supplementary table S9) but no regulation could be observed in both tonic signaling conditions (supplementary table S10). HSP90 inhibition by small-molecule inhibitors has already been reported as cancer treatment option (Nakashima et al., 2010) (reviewed in Taldone et al., 2008; Trepel et al., 2010). Inhibitors of HSP90 such as AT13387 were currently tested in clinical trials for solid tumours where HSP9O inhibition leads to destabilization of growth receptors and kinases including AKT, MAPK, ARAF, and CRAF (Haarberg et al., 2013; Shapiro et al., 2014; Whitesell and Lindquist, 2005). As BRAF is closely related to CRAF and ARAF, HSP90 is most like also a chaperone for BRAF (Haarberg et al., 2013). Furthermore, HSP90 has been identified as a chaperone for the tyrosine kinase ZAP70 in a leukemic disease. As ZAP70 is closely related to SYK, HSP90 could also be a chaperone for SYK (Castro et al., 2005). HSP90 inhibition with PU-H71 also showed good results in DLBCL which is closely related to $\mathrm{BL}$ in terms of antitumor activity (Cerchietti et al., 2009). However, it is not entirely clear if and how BRAF and SYK are involved in tonic signaling, therefore, the finding is preliminary and needs to be further validated.

\subsubsection{MYC}

MYC is a general transcription factor with several gene targets, amongst others CD79B and SYK (Li et al., 2003). MYC translocation is the mutational hallmark of BL and leads to MYC overexpression (reviewed in Ferry, 2006; Schmitz et al., 2012). MYC could not be identified on the protein expression level analysis as described in 3.1.2. In the DG75 BCR stimulation time 
course, three MYC phosphorylation sites could be quantified of which one was of particular interest (MYC T58) as this p-site lies within a phosphodegron leading to MYC degradation after the priming phosphorylation (Welcker et al., 2004; Yada et al., 2004). However, none of the MYC p-sites in any of the experiments identified in this study, including T58 in the BCR stimulation time course in DG75 cells, were found to be regulated (supplementary tables S2). MYC T58 is also not affected by a point mutation that would hinder MYC degradation in the primary patient cell line FFM_BL1. MYC is therefore most likely not directly affected by regulation on the phosphorylation level. MYC activity is context-dependent by heterodimerization with other proteins such as MAX or MIZ-1. After interaction with MAX, the MYC-MAX heterodimer acts as transcription activating complex (reviewed in Cai et al., 2015). MAX possesses two N-terminal p-sites (S2 and S11) which were identified in the antigendependent BCR stimulation time course in DG75 (supplementary table S2). Phosphorylation of both $p$-sites inhibits MAX homodimerization but not MYC-MAX heterodimerization (Berberich and Cole, 1992; Koskinen et al., 1994). MAX homodimers counteract MYC-MAX heterodimers and reduces the amount of available MAX (reviewed in Berg, 2010). Either of the two p-sites was differentially phosphorylated, however, both positions were not differentially phosphorylated at the same time (supplementary table S2). MAX homodimerization might be inhibited thereby favoring MYC-MAX heterodimerization leading to transcriptional activation of MYC target genes. Although MYC itself cannot be targeted by small-molecule inhibitors, there are strategies to indirectly inhibit MYC, for example by repressing its transcription. MYC transcription depends on BRD4, a bromodomain containing protein that binds to lysine acetylated histones and recruits further factors for transcriptional activation of MYC (Albihn et al., 2010; Delmore et al., 2011; Lovén et al., 2013). Inhibition by bromodomain inhibitors of MYC has shown good results even in case MYC is translocated (Lovén et al., 2013). Another strategy is the inhibition of MYC dimerization with its activating interaction partners by Omomyc thereby inhibiting MYC target gene expression (Soucek et al., 2002). Omomyc can dimerize with MYC and does hinder the activation on gene transcription while the transcriptional repression function is maintained (Savino et al., 2011; Soucek et al., 2002) (reviewed in Cai et al., 2015).

\subsubsection{CD37}

The cell surface receptor Leukocyte antigen CD37 (CD37) was identified as a candidate for targeted therapy in Burkitt's lymphoma in this study. CD37 is expressed in mature B cells 
including malignantly transformed B-cells, but not in plasma cells, and is described to be involved in T-cell-B-cell interactions (Knobeloch et al., 2000; Schwartz-Albiez et al., 1988; Tarrant et al., 2003). The CD37 p-site identified in this study lies within an ITAM-like motif and therefore is thought to be phosphorylated by Src family kinases to recruit SYK (Lapalombella et al., 2012). Phosphorylation of this site has been described to drive survival by signaling through the PI3K pathway (Lapalombella et al., 2012). Indeed, the p-site was found to be dephosphorylated upon SYK inhibition, but was upregulated after BCR knockdown (supplementary table S10). Furthermore, SYK was found to be regulated after BCR stimulation in DG75 as described in 3.1.13. The involvement of this p-site was dependent on the genetic context as the phosphorylation behavior was different in the three distinct BL cell lines after BCR stimulation (3.1.13; Figure 3.14; supplementary table S9). Of note, CD37 has already been considered to be a promising target for immunotherapy in B-cell malignancies (Heider et al., 2011). As CD37 could be an additional driver of malignant tonic signaling, knockdown as described in this study for CD79A (2.2.1.3.2) and the CD37 interactome have to be further investigated to validate $\mathrm{CD} 37$ as therapeutic option.

\subsection{Conclusion and perspective}

In combination with other experimental approaches, the phosphoproteome analyses presented in this work under various experimental conditions and in different disease models allowed an in-depth characterization of signal transducing events. In particular, the analysis of distinct cell lines after BCR stimulation showed highly individual responses in cells that cannot be easily distinguished on the histological level. Therefore, MS-based signaling analysis could be a suitable tool for the characterization of patient-derived samples in clinical diagnostics to develop personalized therapeutic approaches. Furthermore, the targeted receptor ablation in combination with mass spectrometry-based phosphoproteomic analysis enabled the investigation of the contribution of the BCR alone on signaling processes. The influence of kinase ablation could be directly investigated on the phosphoproteomic level as well as the involvement of signaling adapters in receptor signaling. The generated datasets may provide a basis for further functional studies of single phosphorylation sites or proteins. The improvement of enrichment methods and the development of new mass spectrometers will in future lead to even higher identification rates and therefore will reach a yet unknown depth of analysis. Database knowledge will also grow and more sophisticated modeling tools will be developed that will altogether lead to the generation of a more detailed picture of 
systems biology. Taken together, this work provides a valuable basis for further functional studies of druggable protein targets by receptor knockdown, interactome analyses, inhibition of proteins with small-molecules, and p-site mutation. 


\section{Bibliography}

Abraham, R.T., and Weiss, A. (2004). Jurkat T cells and development of the T-cell receptor signalling paradigm. Nature Reviews Immunology 4, 301-308.

Albihn, A., Johnsen, J.I., and Henriksson, M.A. (2010). MYC in oncogenesis and as a target for cancer therapies. Advances in Cancer Research 107, 163-224.

Allam, A., Niiro, H., Clark, E.A., and Marshall, A.J. (2004). The adaptor protein Bam32 regulates Rac1 activation and actin remodeling through a phosphorylation-dependent mechanism. J Biol Chem 279, 39775-39782.

Allfrey, V.G., Faulkner, R., and Mirsky, A.E. (1964). ACETYLATION AND METHYLATION OF HISTONES AND THEIR POSSIBLE ROLE IN THE REGULATION OF RNA SYNTHESIS. Proc Natl Acad Sci USA 51, 786-794.

Anders, S., and Huber, W. (2010). Differential expression analysis for sequence count data. Genome Biol 11, R106.

Anderson, K.E., Lipp, P., Bootman, M., Ridley, S.H., Coadwell, J., Rönnstrand, L., Lennartsson, J., Holmes, A.B., Painter, G.F., Thuring, J., et al. (2000). DAPP1 undergoes a PI 3-kinase-dependent cycle of plasma-membrane recruitment and endocytosis upon cell stimulation. Curr Biol 10, 1403-1412.

Ang, X.L., and Harper, J.W. (2005). SCF-mediated protein degradation and cell cycle control. Oncogene 24, 28602870.

Antica, M., Dubravcic, K., Weber, I., Raic, L., Labar, B., and Batinic, D. (2007). A search for a mutation of the Aiolos phosphorylation domain in lymphocytes from patients with leukemia. Haematologica 92, 260-261.

Bahram, F., Lehr, von der, N., Cetinkaya, C., and Larsson, L.G. (2000). c-Myc hot spot mutations in lymphomas result in inefficient ubiquitination and decreased proteasome-mediated turnover. Blood 95, 2104-2110.

Balagopalan, L., Coussens, N.P., Sherman, E., Samelson, L.E., and Sommers, C.L. (2010). The LAT story: a tale of cooperativity, coordination, and choreography. Cold Spring Harbor Perspectives in Biology 2, a005512.

Banerjee, N., and Zhang, M.Q. (2002). Functional genomics as applied to mapping transcription regulatory networks. Current Opinion in Microbiology 5, 313-317.

Bantscheff, M., Eberhard, D., Abraham, Y., Bastuck, S., Boesche, M., Hobson, S., Mathieson, T., Perrin, J., Raida, M., Rau, C., et al. (2007). Quantitative chemical proteomics reveals mechanisms of action of clinical ABL kinase inhibitors. 25, 1035-1044.

Bantscheff, M., Lemeer, S., Savitski, M.M., and Kuster, B. (2012). Quantitative mass spectrometry in proteomics: critical review update from 2007 to the present. Analytical and Bioanalytical Chemistry 404, 939-965.

Bantscheff, M., Schirle, M., Sweetman, G., Rick, J., and Kuster, B. (2007). Quantitative mass spectrometry in proteomics: a critical review. Analytical and Bioanalytical Chemistry 389, 1017-1031.

Batth, T.S., Francavilla, C., and Olsen, J.V. (2014). Off-line high-pH reversed-phase fractionation for in-depth phosphoproteomics. Journal of Proteome Research 13, 6176-6186.

Beausoleil, S.A., Villén, J., Gerber, S.A., and Rush, J. (2006). A probability-based approach for high-throughput protein phosphorylation analysis and site localization. Nature.

Beausoleil, S.A., Jedrychowski, M., Schwartz, D., Elias, J.E., Villen, J., Li, J., Cohn, M.A., Cantley, L.C., and Gygi, S.P. (2004). Large-scale characterization of HeLa cell nuclear phosphoproteins. Proc Natl Acad Sci USA 101, 1213012135 .

Ben-Bassat, H., Polliack, A., Mitrani-Rosenbaum, S., Reichert, F., Froimovici, M., and Goldblum, N. (1977). A comparative study of human cell lines derived from patients with lymphoma, leukemia and infectious mononucleosis: membrane properties, ultrastructure, and surface morphology. Cancer 40, 1481-1491.

Bendall, S.C., Hughes, C., Stewart, M.H., Doble, B., Bhatia, M., and Lajoie, G.A. (2008). Prevention of amino acid conversion in SILAC experiments with embryonic stem cells. Mol Cell Proteomics 7, 1587-1597.

Benjamini, Y., and Hochberg, Y. (1995). Controlling the false discovery rate: a practical and powerful approach to multiple testing. Journal of the Royal Statistical Society. 
Berberich, S.J., and Cole, M.D. (1992). Casein kinase II inhibits the DNA-binding activity of Max homodimers but not Myc/Max heterodimers. Genes \& Development 6, 166-176.

Berg, T. (2010). Small-molecule modulators of c-Myc/Max and Max/Max interactions. Small-Molecule Inhibitors of Protein-Protein Interactions. Volume 348 of the series Current Topics in Microbiology and Immunology pp 139-149.

Bergsagel, P.L., Chesi, M., Nardini, E., Brents, L.A., Kirby, S.L., and Kuehl, W.M. (1996). Promiscuous translocations into immunoglobulin heavy chain switch regions in multiple myeloma. Proc Natl Acad Sci USA 93, 13931-13936.

Bertran-Vicente, J., Serwa, R.A., Schümann, M., Schmieder, P., Krause, E., and Hackenberger, C.P.R. (2014). Sitespecifically phosphorylated lysine peptides. Journal of the American Chemical Society 136, 13622-13628.

Blagoev, B., and Mann, M. (2006). Quantitative proteomics to study mitogen-activated protein kinases. Methods $40,243-250$.

Blum, K.A., Lozanski, G., and Byrd, J.C. (2004). Adult Burkitt leukemia and lymphoma. Blood 104, 3009-3020.

Boersema, P.J., Mohammed, S., and Heck, A.J.R. (2009). Phosphopeptide fragmentation and analysis by mass spectrometry. J Mass Spectrom 44, 861-878.

Bohnenberger, H., Oellerich, T., Engelke, M., Hsiao, H.-H., Urlaub, H., and Wienands, J. (2011). Complex phosphorylation dynamics control the composition of the Syk interactome in B cells. Eur J Immunol 41, 15501562.

Bondarenko, P.V., Chelius, D., and Shaler, T.A. (2002). Identification and Relative Quantitation of Protein Mixtures by Enzymatic Digestion Followed by Capillary Reversed-Phase Liquid Chromatography-Tandem Mass Spectrometry. Analytical Chemistry 74, 4741.

Bories, J.C., Willerford, D.M., Grévin, D., Davidson, L., Camus, A., Martin, P., Stéhelin, D., and Alt, F.W. (1995). Increased T-cell apoptosis and terminal B-cell differentiation induced by inactivation of the Ets-1 protooncogene. Nature 377, 635-638.

Borst, J., Jacobs, H., and Brouns, G. (1996). Composition and function of T-cell receptor and B-cell receptor complexes on precursor lymphocytes. Current Opinion in Immunology 8, 181-190.

Brack, C., Hirama, M., Lenhard-Schuller, R., and Tonegawa, S. (1978). A complete immunoglobulin gene is created by somatic recombination. Cell 15, 1-14.

Bramblett, G.T., Goedert, M., Jakes, R., Merrick, S.E., and Trojanowski, J.Q. (1993). Abnormal tau phosphorylation at Ser396 in alzheimer's disease recapitulates development and contributes to reduced microtubule binding. Neuron 10, 1089.

Brose, M.S., Volpe, P., Feldman, M., Kumar, M., Rishi, I., Gerrero, R., Einhorn, E., Herlyn, M., Minna, J., Nicholson, A., et al. (2002). BRAF and RAS mutations in human lung cancer and melanoma. Cancer Research 62, 6997-7000.

Brownridge, P., Xia, D., Mackay, K., Gonzalez-Galarza, F.F., Kenyani, J., Harman, V., Beynon, R.J., and Jones, A.R. (2012). A software toolkit and interface for performing stable isotope labeling and top3 quantification using Progenesis LC-MS. OMICS 16, 489-495.

Burack, W.R., and Sturgill, T.W. (1997). The activating dual phosphorylation of MAPK by MEK is nonprocessive. Biochemistry 36, 5929.

Burkitt, D. (1958). A sarcoma involving the jaws in African children. Br J Surg 46, 218-223.

Burkitt, D. (1962). Determining the climatic limitations of a children's cancer common in Africa. Br Med J 2, 10191023.

Burkitt, D.P. (1961). Observations on the geography of malignant lymphoma. East Afr Med J 38, 511-514.

Burnett, G., and Kennedy, E.P. (1954). The enzymatic phosphorylation of proteins. J Biol Chem 211, 969-980.

Byrd, J.C., Brown, J.R., O'Brien, S., Barrientos, J.C., Kay, N.E., Reddy, N.M., Coutre, S., Tam, C.S., Mulligan, S.P., Jaeger, U., et al. (2014). Ibrutinib versus ofatumumab in previously treated chronic lymphoid leukemia. N Engl J Med 371, 213-223.

Cai, Q., Medeiros, L.J., Xu, X., and Young, K.H. (2015). MYC-driven aggressive B-cell lymphomas: biology, entity, differential diagnosis and clinical management. Oncotarget 6, 38591-38616. 
Caldon, C.E., Lee, C.S.L., Sutherland, R.L., and Musgrove, E.A. (2007). Wilms' tumor protein 1: an early target of progestin regulation in T-47D breast cancer cells that modulates proliferation and differentiation. Oncogene 27, 126-138.

Campo, E., Swerdlow, S.H., Harris, N.L., Pileri, S., Stein, H., and Jaffe, E.S. (2011). The 2008 WHO classification of lymphoid neoplasms and beyond: evolving concepts and practical applications. Blood 117, 5019-5032.

Canagarajah, B.J., Khokhlatchev, A., Cobb, M.H., and Goldsmith, E.J. (1997). Activation mechanism of the MAP kinase ERK2 by dual phosphorylation. Cell 90, 859-869.

Cao, C., Li, Y., Leng, Y., Li, P., Ma, Q., and Kufe, D. (2005). Ubiquitination and degradation of the Arg tyrosine kinase is regulated by oxidative stress. Oncogene $24,2433-2440$.

Cao, L., Ding, Y., Hung, N., Yu, K., Ritz, A., Raphael, B.J., and Salomon, A.R. (2012). Quantitative phosphoproteomics reveals SLP-76 dependent regulation of PAG and Src family kinases in T cells. PLoS ONE 7, e46725.

Capdeville, R., Buchdunger, E., Zimmermann, J., and Matter, A. (2002). Glivec (STI571, imatinib), a rationally developed, targeted anticancer drug. Nature Reviews Drug Discovery 1, 493-502.

Carissimi, C., Saieva, L., Gabanella, F., and Pellizzoni, L. (2006). Gemin8 is required for the architecture and function of the survival motor neuron complex. J Biol Chem 281, 37009-37016.

Carter, R., and Fearon, D. (1992). CD19: lowering the threshold for antigen receptor stimulation of B lymphocytes. Science 256, 105.

Casteel, D.E., Zhuang, S., Gudi, T., Tang, J., Vuica, M., Desiderio, S., and Pilz, R.B. (2002). cGMP-dependent protein kinase I beta physically and functionally interacts with the transcriptional regulator TFII-I. J Biol Chem 277, 3200332014.

Castro, J.E., Prada, C.E., Loria, O., Kamal, A., Chen, L., Burrows, F.J., and Kipps, T.J. (2005). ZAP-70 is a novel conditional heat shock protein 90 (Hsp90) client: inhibition of Hsp90 leads to ZAP-70 degradation, apoptosis, and impaired signaling in chronic lymphocytic leukemia. Blood 106, 2506-2512.

Cerchietti, L.C., Lopes, E.C., Yang, S.N., Hatzi, K., Bunting, K.L., Tsikitas, L.A., Mallik, A., Robles, A.I., Walling, J., Varticovski, L., et al. (2009). A purine scaffold Hsp90 inhibitor destabilizes BCL-6 and has specific antitumor activity in BCL-6-dependent B cell lymphomas. Nature Medicine 15, 1369-1376.

Chakraborty, A.K., and Weiss, A. (2014). Insights into the initiation of TCR signaling. Nature Immunology 15, 798807.

Chang, L., and Karin, M. (2001). Mammalian MAP kinase signalling cascades. Nature 410, 37-40.

Chaudhuri, R., Sadrieh, A., Hoffman, N.J., Parker, B.L., Humphrey, S.J., Stöckli, J., Hill, A.P., James, D.E., and Yang, J.Y.H. (2015). PhosphOrtholog: a web-based tool for cross-species mapping of orthologous protein posttranslational modifications. BMC Genomics 16, 617.

Chen, L., Monti, S., Juszczynski, P., Daley, J., Chen, W., Witzig, T.E., Habermann, T.M., Kutok, J.L., and Shipp, M.A. (2007). SYK-dependent tonic B-cell receptor signaling is a rational treatment target in diffuse large B-cell lymphoma. Blood 111, 2230-2237.

Chen, S.-J., Shen, Y., and Chen, Z. (2013). A panoramic view of acute myeloid leukemia. Nature Genetics 45, 586587.

Cheung, M., Sharma, A., Madhunapantula, S.V., and Robertson, G.P. (2008). Akt3 and Mutant V600EB-Raf Cooperate to Promote Early Melanoma Development. Cancer Research 68, 3429.

Chong, H., Lee, J., and Guan, K.L. (2001). Positive and negative regulation of Raf kinase activity and function by phosphorylation. The EMBO Journal 20, 3716-3727.

Choudhary, C., Kumar, C., Gnad, F., Nielsen, M.L., Rehman, M., Walther, T.C., Olsen, J.V., and Mann, M. (2009). Lysine acetylation targets protein complexes and co-regulates major cellular functions. Science 325, 834-840.

Chylek, L.A., Akimov, V., Dengjel, J., Rigbolt, K.T.G., Hlavacek, W.S., and Blagoev, B. (2014). Phosphorylation site dynamics of early T-cell receptor signaling. PLoS ONE 9, e104240.

Cieśla, J., Frączyk, T., and Rode, W. (2011). Phosphorylation of basic amino acid residues in proteins: important but easily missed. Acta Biochimica Polonica. 
Cohen, M.S., Zhang, C., Shokat, K.M., and Taunton, J. (2005). Structural bioinformatics-based design of selective, irreversible kinase inhibitors. Science 308, 1318-1321.

Cohen, P. (2002). Protein kinases--the major drug targets of the twenty-first century? Nature Reviews Drug Discovery 1, 309-315.

Cohen, P., and Alessi, D.R. (2012). Kinase drug discovery - What's next in the field? ACS Chemical Biology 8, 96104.

Coon, J.J., Shabanowitz, J., Hunt, D.F., and Syka, J.E.P. (2005). Electron transfer dissociation of peptide anions. Journal of the American Society for Mass Spectrometry 16, 880-882.

Cox, J., and Mann, M. (2008). MaxQuant enables high peptide identification rates, individualized p.p.b.-range mass accuracies and proteome-wide protein quantification. Nature Biotechnology 26, 1367-1372.

Cox, J., Hein, M.Y., Luber, C.A., Paron, I., Nagaraj, N., and Mann, M. (2014). Accurate proteome-wide label-free quantification by delayed normalization and maximal peptide ratio extraction, termed MaxLFQ. Mol Cell Proteomics 13, 2513-2526.

Cox, J., Neuhauser, N., Michalski, A., Scheltema, R.A., Olsen, J.V., and Mann, M. (2011). Andromeda: a peptide search engine integrated into the MaxQuant environment. Journal of Proteome Research 10, 1794-1805.

Cozzi, M., Giorgi, F., Marcelli, E., Pentimalli, F., Forte, I.M., Schenone, S., D’Urso, V., De Falco, G., Botta, M., Giordano, A., et al. (2012). Antitumor activity of new pyrazolo[3,4- d ]pyrimidine SRC kinase inhibitors in Burkitt lymphoma cell lines and its enhancement by WEE1 inhibition. Cell Cycle 11, 1029.

Cozzone, A.J. (1988). Protein phosphorylation in prokaryotes. Annu Rev Microbiol 42, 97-125.

Czibere, A., Grall, F., and Aivado, M. (2006). Perspectives of proteomics in acute myeloid leukemia. Expert Review of Anticancer Therapy 6, 1663-1675.

Dalla-Favera, R., Bregni, M., Erikson, J., Patterson, D., Gallo, R.C., and Croce, C.M. (1982). Human c-myc onc gene is located on the region of chromosome 8 that is translocated in Burkitt lymphoma cells. Proc Natl Acad Sci USA 79, 7824-7827.

Dang, C.V. (2012). MYC on the path to cancer. Cell 149, 22-35.

Dave, S.S., Fu, K., Wright, G.W., Lam, L.T., Kluin, P., Boerma, E.-J., Greiner, T.C., Weisenburger, D.D., Rosenwald, A., Ott, G., et al. (2006). Molecular diagnosis of Burkitt's lymphoma. N Engl J Med 354, 2431-2442.

Davies, H., Bignell, G.R., Cox, C., Stephens, P., Edkins, S., Clegg, S., Teague, J., Woffendin, H., Garnett, M.J., Bottomley, W., et al. (2002). Mutations of the BRAF gene in human cancer. Nature 417, 949-954.

Davis, R.E., Ngo, V.N., Lenz, G., Tolar, P., Young, R.M., Romesser, P.B., Kohlhammer, H., Lamy, L., Zhao, H., Yang, Y., et al. (2010). Chronic active B-cell-receptor signalling in diffuse large B-cell lymphoma. Nature 463, 88-92.

Deckert, M., Elly, C., Altman, A., and Liu, Y.C. (1998). Coordinated regulation of the tyrosine phosphorylation of Cbl by Fyn and Syk tyrosine kinases. J Biol Chem 273, 8867-8874.

Delmore, J.E., Issa, G.C., Lemieux, M.E., Rahl, P.B., Shi, J., Jacobs, H.M., Kastritis, E., Gilpatrick, T., Paranal, R.M., Qi, J., et al. (2011). BET bromodomain inhibition as a therapeutic strategy to target c-Myc. Cell 146, 904-917.

Denépoux, S., Razanajaona, D., Blanchard, D., Meffre, G., Capra, J.D., Banchereau, J., and Lebecque, S. (1997). Induction of somatic mutation in a human B cell line in vitro. Immunity 6, 35-46.

Dephoure, N., and Gygi, S.P. (2012). Hyperplexing: a method for higher-order multiplexed quantitative proteomics provides a map of the dynamic response to rapamycin in yeast. Science Signaling 5, rs2.

Devkota, S., Joseph, R.E., Min, L., Fulton, D.B., and Andreotti, A.H. (2015). Scaffold Protein SLP-76 Primes PLC 1 for Activation by ITK-Mediated Phosphorylation. J Mol Biol 427, 2734-2747.

Dhillon, A.S., Hagan, S., Rath, O., and Kolch, W. (2007). MAP kinase signalling pathways in cancer. Oncogene 26, 3279-3290.

Di Bartolo, V., Montagne, B., Salek, M., Jungwirth, B., Carrette, F., Fourtane, J., Sol-Foulon, N., Michel, F., Schwartz, O., Lehmann, W.D., et al. (2007). A novel pathway down-modulating T cell activation involves HPK-1dependent recruitment of 14-3-3 proteins on SLP-76. J Exp Med 204, 681-691. 
Dole, M., Hines, R.L., Mack, L.L., Mobley, R.C., Ferguson, L.D., and Alice, M.B. (1968). Gas Phase Macroions. Macromolecules 1, 96.

Eckhart, W., Hutchinson, M.A., and Hunter, T. (1979). An activity phosphorylating tyrosine in polyoma T antigen immunoprecipitates. Cell 18, 925-933.

Egertson, J.D., Kuehn, A., Merrihew, G.E., Bateman, N.W., MacLean, B.X., Ting, Y.S., Canterbury, J.D., Marsh, D.M., Kellmann, M., Zabrouskov, V., et al. (2013). Multiplexed MS/MS for improved data-independent acquisition. Nature Methods 10, 744-746.

Elias, J.E., and Gygi, S.P. (2007). Target-decoy search strategy for increased confidence in large-scale protein identifications by mass spectrometry. Nature Methods 4, 207-214.

Elsholz, A.K.W., Turgay, K., Michalik, S., Hessling, B., Gronau, K., Oertel, D., Mäder, U., Bernhardt, J., Becher, D., Hecker, M., et al. (2012). Global impact of protein arginine phosphorylation on the physiology of Bacillus subtilis. Proc Natl Acad Sci USA 109, 7451-7456.

Engels, N., König, L.M., Heemann, C., Lutz, J., Tsubata, T., Griep, S., Schrader, V., and Wienands, J. (2009). Recruitment of the cytoplasmic adaptor Grb2 to surface IgG and IgE provides antigen receptor-intrinsic costimulation to class-switched B cells. Nature Immunology 10, 1018-1025.

Epstein, M.A., and Barr, Y.M. (1964). CULTIVATION IN VITRO OF HUMAN LYMPHOBLASTS FROM BURKITT'S MALIGNANT LYMPHOMA. The Lancet 283, 252.

Estey, E., and Döhner, H. (2006). Acute myeloid leukaemia. The Lancet.

Evan, G.I., Wyllie, A.H., Gilbert, C.S., Littlewood, T.D., Land, H., Brooks, M., Waters, C.M., Penn, L.Z., and Hancock, D.C. (1992). Induction of apoptosis in fibroblasts by c-myc protein. Cell 69, 119-128.

Feederle, R., Delecluse, H.-J., Rouault, J.-P., Schepers, A., and Hammerschmidt, W. (2004). Efficient somatic gene targeting in the lymphoid human cell line DG75. Gene 343, 91-97.

Felsher, D.W., and Bishop, J.M. (1999). Transient excess of MYC activity can elicit genomic instability and tumorigenesis. Proc Natl Acad Sci USA 96, 3940-3944.

Fenn, J., Mann, M., Meng, C., Wong, S., and Whitehouse, C. (1989). Electrospray ionization for mass spectrometry of large biomolecules. Science 246, 64.

Fenn, J.B., Mann, M., Meng, C.K., Wong, S.F., and Whitehouse, C.M. (1990). Electrospray ionization-principles and practice. Mass Spectrometry Reviews 9, 37.

Ferry, J.A. (2006). Burkitt's lymphoma: clinicopathologic features and differential diagnosis. The Oncologist 11, 375-383.

Ficarro, S.B., McCleland, M.L., Stukenberg, P.T., Burke, D.J., Ross, M.M., Shabanowitz, J., Hunt, D.F., and White, F.M. (2002). Phosphoproteome analysis by mass spectrometry and its application to Saccharomyces cerevisiae. Nature Biotechnology 20, 301-305.

Fiil, B.K., and Gyrd-Hansen, M. (2014). Met1-linked ubiquitination in immune signalling. FEBS J 281, 4337-4350.

Flaherty, K.T., Puzanov, I., Kim, K.B., Ribas, A., McArthur, G.A., Sosman, J.A., O'Dwyer, P.J., Lee, R.J., Grippo, J.F., Nolop, K., et al. (2010). Inhibition of mutated, activated BRAF in metastatic melanoma. N Engl J Med 363, 809819.

Frese, C.K., Altelaar, A.F.M., Hennrich, M.L., Nolting, D., Zeller, M., Griep-Raming, J., Heck, A.J.R., and Mohammed, S. (2011). Improved peptide identification by targeted fragmentation using CID, HCD and ETD on an LTQ-Orbitrap Velos. Journal of Proteome Research 10, 2377-2388.

Frese, C.K., Altelaar, A.F.M., van den Toorn, H., Nolting, D., Griep-Raming, J., Heck, A.J.R., and Mohammed, S. (2012). Toward full peptide sequence coverage by dual fragmentation combining electron-transfer and higherenergy collision dissociation tandem mass spectrometry. Analytical Chemistry 84, 9668-9673.

Frese, C.K., Zhou, H., Taus, T., Altelaar, A.F.M., Mechtler, K., Heck, A.J.R., and Mohammed, S. (2013). Unambiguous phosphosite localization using electron-transfer/higher-energy collision dissociation (EThcD). Journal of Proteome Research 12, 1520-1525.

Fu, C., Turck, C.W., Kurosaki, T., and Chan, A.C. (1998). BLNK: a central linker protein in B cell activation. Immunity 9, 93-103. 
Fujimoto, M., Fujimoto, Y., Poe, J.C., Jansen, P.J., Lowell, C.A., DeFranco, A.L., and Tedder, T.F. (2000). CD19 regulates Src family protein tyrosine kinase activation in B lymphocytes through processive amplification. Immunity 13, 47-57.

Fujita, S., Buziba, N., Kumatori, A., Senba, M., Yamaguchi, A., and Toriyama, K. (2004). Early stage of Epstein-Barr virus lytic infection leading to the "starry sky" pattern formation in endemic Burkitt lymphoma. Arch Pathol Lab Med 128, 549-552.

Fukuda, I., Hirabayashi-Ishioka, Y., Sakikawa, I., Ota, T., Yokoyama, M., Uchiumi, T., and Morita, A. (2013). Optimization of Enrichment Conditions on $\mathrm{TiO}_{2}$ Chromatography Using Glycerol As an Additive Reagent for Effective Phosphoproteomic Analysis. Journal of Proteome Research 12, 5587.

Gabay, C., Ben-Bassat, H., Schlesinger, M., and Laskov, R. (1999). Somatic mutations and intraclonal variations in the rearranged Vk genes of B-non-Hodgkin's lymphoma cell lines. European Journal of ... 63, 180.

Gao, F., Foat, B.C., and Bussemaker, H.J. (2004). Defining transcriptional networks through integrative modeling of mRNA expression and transcription factor binding data. BMC Bioinformatics 5, 31.

Garcia, K.C., and Adams, E.J. (2005). How the T cell receptor sees antigen--a structural view. Cell 122, 333-336.

Geiger, T., Cox, J., and Mann, M. (2010). Proteomics on an Orbitrap benchtop mass spectrometer using all-ion fragmentation. Mol Cell Proteomics 9, 2252-2261.

Gibson, A.W., Cheng, T., and Johnston, R.N. (1995). Apoptosis induced by c-myc overexpression is dependent on growth conditions. Experimental Cell Research 218, 351-358.

Gillig, K.J., Bluhm, B.K., and Russell, D.H. (1996). Ion motion in a Fourier transform ion cyclotron resonance wire ion guide cell. International Journal of Mass Spectrometry and Ion Processes 157-158, 129.

Glish, G.L., and Burinsky, D.J. (2007). Hybrid mass spectrometers for tandem mass spectrometry. Journal of the American Society for Mass Spectrometry 19, 161-172.

Goedert, M. (2015). Alzheimer's and Parkinson's diseases: The prion concept in relation to assembled $A \beta$, tau, and $\alpha$-synuclein. Science 349, 1255555.

Gray, D., and Skarvall, H. (1988). B-cell memory is short-lived in the absence of antigen. Nature 336, 70-73.

Grimsrud, P.A., Swaney, D.L., Wenger, C.D., Beauchene, N.A., and Coon, J.J. (2010). Phosphoproteomics for the masses. ACS Chemical Biology 5, 105-119.

Gross, J.H. (2011). Mass spectrometry-A Textbook, 2nd Edition (Springer).

Gruhler, A., Olsen, J.V., Mohammed, S., Mortensen, P., Faergeman, N.J., Mann, M., and Jensen, O.N. (2005). Quantitative phosphoproteomics applied to the yeast pheromone signaling pathway. Mol Cell Proteomics 4, 310-327.

Gu, W., and Roeder, R.G. (1997). Activation of p53 sequence-specific DNA binding by acetylation of the p53 Cterminal domain. Cell 90, 595-606.

Guan, K.L., Figueroa, C., Brtva, T.R., Zhu, T., Taylor, J., Barber, T.D., and Vojtek, A.B. (2000). Negative regulation of the serine/threonine kinase B-Raf by Akt. J Biol Chem 275, 27354-27359.

Guy, C.S., Vignali, K.M., Temirov, J., Bettini, M.L., Overacre, A.E., Smeltzer, M., Zhang, H., Huppa, J.B., Tsai, Y.-H., Lobry, C., et al. (2013). Distinct TCR signaling pathways drive proliferation and cytokine production in T cells. Nature Immunology 14, 262-270.

Haarberg, H.E., Paraiso, K.H.T., Wood, E., Rebecca, V.W., Sondak, V.K., Koomen, J.M., and Smalley, K.S.M. (2013). Inhibition of Wee1, AKT, and CDK4 underlies the efficacy of the HSP90 inhibitor XL888 in an in vivo model of NRAS-mutant melanoma. Mol Cancer Ther 12, 901-912.

Hahn, C.K., Berchuck, J.E., Ross, K.N., Kakoza, R.M., Clauser, K., Schinzel, A.C., Ross, L., Galinsky, I., Davis, T.N., Silver, S.J., et al. (2009). Proteomic and genetic approaches identify Syk as an AML target. Cancer Cell 16, 281294.

Hall, K.J., Jones, M.L., and Poole, A.W. (2007). Coincident regulation of PKCdelta in human platelets by phosphorylation of Tyr311 and Tyr565 and phospholipase C signalling. Biochem J 406, 501-509. 
Haluska, F.G., TsuJIMOTO, Y., and Croce, C.M. (1987). The $t(8 ; 14)$ chromosome translocation of the Burkitt lymphoma cell line Daudi occurred during immunoglobulin gene rearrangement and involved the heavy chain diversity region. Proc Natl Acad Sci USA 84, 6835-6839.

Hansen, K.D., Long, L., Gentleman, R., Falcon, S., Hahne, F., and Sarkar, D. (2010). Rgraphviz: Provides plotting capabilities for R graph objects.

Hari, S.B., Merritt, E.A., and Maly, D.J. (2013). Sequence determinants of a specific inactive protein kinase conformation. Chemistry \& Biology 20(6), 806-815.

Harwood, N.E., and Batista, F.D. (2011). The cytoskeleton coordinates the early events of B-cell activation. Cold Spring Harbor Perspectives in Biology 3, a002360.

Hatzivassiliou, G., Song, K., Yen, I., Brandhuber, B.J., Anderson, D.J., Alvarado, R., Ludlam, M.J.C., Stokoe, D., Gloor, S.L., Vigers, G., et al. (2010). RAF inhibitors prime wild-type RAF to activate the MAPK pathway and enhance growth. Nature 464, 431-435.

Hayakawa, F., Towatari, M., Kiyoi, H., Tanimoto, M., Kitamura, T., Saito, H., and Naoe, T. (2000). Tandemduplicated Flt3 constitutively activates STAT5 and MAP kinase and introduces autonomous cell growth in IL-3dependent cell lines. Oncogene 19, 624-631.

Heider, K.-H., Kiefer, K., Zenz, T., Volden, M., Stilgenbauer, S., Ostermann, E., Baum, A., Lamche, H., Küpcü, Z., Jacobi, A., et al. (2011). A novel Fc-engineered monoclonal antibody to CD37 with enhanced ADCC and high proapoptotic activity for treatment of B-cell malignancies. Blood 118, 4159-4168.

Hess, J.F., Bourret, R.B., and Simon, M.I. (1988). Histidine phosphorylation and phosphoryl group transfer in bacterial chemotaxis. Nature 336, 139-143.

Hicke, L., Hicke, L., Schubert, H.L., Hill, C.P., Schubert, H.L., and Hill, C.P. (2005). Ubiquitin-binding domains. Nature Reviews Molecular Cell Biology 6, 610-621.

Hitosugi, T., Zhou, L., Elf, S., Fan, J., Kang, H.-B., Seo, J.H., Shan, C., Dai, Q., Zhang, L., Xie, J., et al. (2012). Phosphoglycerate mutase 1 coordinates glycolysis and biosynthesis to promote tumor growth. Cancer Cell 22, 585-600.

Hoelzer, D., Walewski, J., Döhner, H., Viardot, A., Hiddemann, W., Spiekermann, K., Serve, H., Dührsen, U., Hüttmann, A., Thiel, E., et al. (2014). Improved outcome of adult Burkitt lymphoma/leukemia with rituximab and chemotherapy: report of a large prospective multicenter trial. Blood 124, 3870-3879.

Holmberg, C.I., Tran, S.E.F., Eriksson, J.E., and Sistonen, L. (2002). Multisite phosphorylation provides sophisticated regulation of transcription factors. Trends Biochem Sci 27, 619-627.

Hong, J., Hu, K., Yuan, Y., Sang, Y., Bu, Q., Chen, G., Yang, L., Li, B., Huang, P., Chen, D., et al. (2012). CHK1 targets spleen tyrosine kinase (L) for proteolysis in hepatocellular carcinoma. J Clin Invest 122, 2165-2175.

Huang, P.H., Mukasa, A., Bonavia, R., Flynn, R.A., Brewer, Z.E., Cavenee, W.K., Furnari, F.B., and White, F.M. (2007). Quantitative analysis of EGFRvIII cellular signaling networks reveals a combinatorial therapeutic strategy for glioblastoma. Proc Natl Acad Sci USA 104, 12867-12872.

Huang, Y.H., and Sauer, K. (2010). Lipid signaling in T-cell development and function. Cold Spring Harbor Perspectives in Biology 2, a002428.

Hunter, T. (1995). Protein kinases and phosphatases: the yin and yang of protein phosphorylation and signaling. Cell.

Hunter, T. (2007). The age of crosstalk: phosphorylation, ubiquitination, and beyond. Molecular Cell 28, 730-738.

Hunter, T. (2014). The genesis of tyrosine phosphorylation. Cold Spring Harbor Perspectives in Biology 6, a020644.

Hunter, T., and Karin, M. (1992). The regulation of transcription by phosphorylation. Cell 70, 375.

Hwang, C.-S., Shemorry, A., and Varshavsky, A. (2010). N-terminal acetylation of cellular proteins creates specific degradation signals. Science 327, 973-977.

Hwang, S., Palin, A.C., Li, L., Song, K.-D., Lee, J., Herz, J., Tubo, N., Chu, H., Pepper, M., Lesourne, R., et al. (2015). TCR ITAM multiplicity is required for the generation of follicular helper T-cells. Nat Commun 6, 6982. 
Iliuk, A.B., Martin, V.A., Alicie, B.M., Geahlen, R.L., and Tao, W.A. (2010). In-depth analyses of kinase-dependent tyrosine phosphoproteomes based on metal ion-functionalized soluble nanopolymers. Mol Cell Proteomics 9 , 2162-2172.

Iwai, K., and Tokunaga, F. (2009). Linear polyubiquitination: a new regulator of NF-kappaB activation. EMBO Reports 10, 706-713.

Jedrychowski, M.P., Huttlin, E.L., Haas, W., Sowa, M.E., Rad, R., and Gygi, S.P. (2011). Evaluation of HCD- and CIDtype fragmentation within their respective detection platforms for murine phosphoproteomics. Mol Cell Proteomics 10, M111.009910.

Jensen, L.J., Jensen, T.S., de Lichtenberg, U., Brunak, S., and Bork, P. (2006). Co-evolution of transcriptional and post-translational cell-cycle regulation. Nature 443, 594-597.

Jin, J., and Pawson, T. (2012). Modular evolution of phosphorylation-based signalling systems. Philos Trans R Soc Lond, B, Biol Sci 367, 2540-2555.

Johnson, D.S., Mortazavi, A., Myers, R.M., and Wold, B. (2007). Genome-wide mapping of in vivo protein-DNA interactions. Science 316, 1497-1502.

Johnson, K.D., Christensen, H.M., Zhao, B., and Bresnick, E.H. (2001). Distinct mechanisms control RNA polymerase II recruitment to a tissue-specific locus control region and a downstream promoter. Molecular Cell $8,465-471$.

Kalter, S.P., Riggs, S.A., Cabanillas, F., Butler, J.J., Hagemeister, F.B., Mansell, P.W., Newell, G.R., Velasquez, W.S., Salvador, P., and Barlogie, B. (1985). Aggressive non-Hodgkin's lymphomas in immunocompromised homosexual males. Blood 66, 655-659.

Kambayashi, T., Allenspach, E.J., Chang, J.T., Zou, T., Shoag, J.E., Reiner, S.L., Caton, A.J., and Koretzky, G.A. (2009). Inducible MHC class II expression by mast cells supports effector and regulatory T cell activation. J Immunol 182, 4686-4695.

Kanazawa, S., Soucek, L., Evan, G., Okamoto, T., and Peterlin, B.M. (2003). c-Myc recruits P-TEFb for transcription, cellular proliferation and apoptosis. Oncogene 22, 5707-5711.

Karas, M., Bahr, U., and Dülcks, T. (2000). Nano-electrospray ionization mass spectrometry: addressing analytical problems beyond routine. Fresenius' Journal of Analytical Chemistry 366, 669.

Kim, M.Y., Woo, E.M., Chong, Y.T.E., Homenko, D.R., and Kraus, W.L. (2006a). Acetylation of estrogen receptor alpha by 300 at lysines 266 and 268 enhances the deoxyribonucleic acid binding and transactivation activities of the receptor. Mol Endocrinol 20, 1479-1493.

Kim, M.-S., Pinto, S.M., Getnet, D., Nirujogi, R.S., Manda, S.S., Chaerkady, R., Madugundu, A.K., Kelkar, D.S., Isserlin, R., Jain, S., et al. (2014). A draft map of the human proteome. Nature 509, 575-581.

Kim, S.C., Sprung, R., Chen, Y., Xu, Y., Ball, H., Pei, J., Cheng, T., Kho, Y., Xiao, H., Xiao, L., et al. (2006b). Substrate and functional diversity of lysine acetylation revealed by a proteomics survey. Molecular Cell 23, 607-618.

Kirkpatrick, D.S., Denison, C., and Gygi, S.P. (2005a). Weighing in on ubiquitin: the expanding role of massspectrometry-based proteomics. Nature Cell Biology 7, 750-757.

Kirkpatrick, D.S., Weldon, S.F., Tsaprailis, G., Liebler, D.C., and Gandolfi, A.J. (2005b). Proteomic identification of ubiquitinated proteins from human cells expressing His-tagged ubiquitin. Proteomics 5, 2104-2111.

Kishimoto, T., Ralph, P., and Good, R.A. (1977). Stimulation of a human B-lymphocyte line by antiimmunoglobulin and its concanavalin A-induced suppression by a T-cell line. Clin Exp Immunol 30, 62-69.

Klein, E., Klein, G., Nadkarni, J.S., Nadkarni, J.J., Wigzell, H., and Clifford, P. (1968). Surface IgM-kappa specificity on a Burkitt lymphoma cell in vivo and in derived culture lines. Cancer Research 28, 1300-1310.

Klein, U., and Dalla-Favera, R. (2007). Germinal centres: role in B-cell physiology and malignancy. Nature Reviews Immunology 8, 22-33.

Knobeloch, K.P., Wright, M.D., Ochsenbein, A.F., Liesenfeld, O., Löhler, J., Zinkernagel, R.M., Horak, I., and Orinska, Z. (2000). Targeted inactivation of the tetraspanin CD37 impairs T-cell-dependent B-cell response under suboptimal costimulatory conditions. Mol Cell Biol 20, 5363-5369. 
Koeffler, H., and Golde, D. (1978). Acute myelogenous leukemia: a human cell line responsive to colonystimulating activity. Science 200, 1153.

Kolanczyk, M., Pech, M., Zemojtel, T., Yamamoto, H., Mikula, I., Calvaruso, M.-A., van den Brand, M., Richter, R., Fischer, B., Ritz, A., et al. (2010). NOA1 is an essential GTPase required for mitochondrial protein synthesis. Mol Biol Cell 22, 1-11.

Konermann, L., Ahadi, E., Rodriguez, A.D., and Vahidi, S. (2012). Unraveling the mechanism of electrospray ionization. Analytical Chemistry 85, 2-9.

Koretzky, G.A., Koretzky, G.A., Abtahian, F., Abtahian, F., Silverman, M.A., and Silverman, M.A. (2006). SLP76 and SLP65: complex regulation of signalling in lymphocytes and beyond. Nature Reviews Immunology 6, 67-78.

Koskinen, P.J., Västrik, I., Mäkelä, T.P., Eisenman, R.N., and Alitalo, K. (1994). Max activity is affected by phosphorylation at two NH2-terminal sites. Cell Growth \& Differentiation 5, 313-320.

Kraus, M., Alimzhanov, M.B., Rajewsky, N., and Rajewsky, K. (2004). Survival of resting mature B lymphocytes depends on BCR signaling via the Igalpha/beta heterodimer. Cell 117, 787-800.

Kubo, T., Uchida, Y., Watanabe, Y., Abe, M., Nakamura, A., Ono, M., Akira, S., and Takai, T. (2009). Augmented TLR9-induced Btk activation in PIR-B-deficient B-1 cells provokes excessive autoantibody production and autoimmunity. J Exp Med 206, 1971-1982.

Kulathu, Y., and Komander, D. (2012). Atypical ubiquitylation - the unexplored world of polyubiquitin beyond Lys48 and Lys63 linkages. Nature Reviews Molecular Cell Biology 13, 508-523.

Kumbrink, J., Gerlinger, M., and Johnson, J.P. (2005). Egr-1 induces the expression of its corepressor nab2 by activation of the nab2 promoter thereby establishing a negative feedback loop. J Biol Chem 280, 42785-42793.

Kurosaki, T., Shinohara, H., and Baba, Y. (2009). B cell signaling and fate decision. Annual Review of Immunology 28, 21-55.

Küppers, R. (2005). Mechanisms of B-cell lymphoma pathogenesis. Nature Reviews Cancer 5, 251-262.

Lam, K.P., Kühn, R., and Rajewsky, K. (1997). In vivo ablation of surface immunoglobulin on mature B cells by inducible gene targeting results in rapid cell death. Cell 90, 1073-1083.

Lapalombella, R., Yeh, Y.-Y., Wang, L., Ramanunni, A., Rafiq, S., Jha, S., Staubli, J., Lucas, D.M., Mani, R., Herman, S.E.M., et al. (2012). Tetraspanin CD37 directly mediates transduction of survival and apoptotic signals. Cancer Cell 21, 694-708.

Larsen, M.R., Trelle, M.B., Thingholm, T.E., and Jensen, O.N. (2006). Analysis of posttranslational modifications of proteins by tandem mass spectrometry. Biotechniques 40, 790-798.

Lee, T.I., and Young, R.A. (2013). Transcriptional regulation and its misregulation in disease. Cell 152, $1237-1251$.

L'Hernault, S.W., and Rosenbaum, J.L. (1985). Chlamydomonas alpha-tubulin is posttranslationally modified by acetylation on the epsilon-amino group of a lysine. Biochemistry $24,473-478$.

Larsen, M.R., Thingholm, T.E., Jensen, O.N., Roepstorff, P., and Jørgensen, T.J.D. (2005). Highly selective enrichment of phosphorylated peptides from peptide mixtures using titanium dioxide microcolumns. Mol Cell Proteomics 4, 873-886.

Lasserre, R., Cuche, C., Blecher-Gonen, R., Libman, E., Biquand, E., Danckaert, A., Yablonski, D., Alcover, A., and Di Bartolo, V. (2011). Release of serine/threonine-phosphorylated adaptors from signaling microclusters downregulates T cell activation. J Cell Biol 195, 839-853.

Le Bras, S., Foucault, I., Foussat, A., Brignone, C., Acuto, O., and Deckert, M. (2004). Recruitment of the actinbinding protein HIP-55 to the immunological synapse regulates T cell receptor signaling and endocytosis. J Biol Chem 279, 15550-15560.

Lekmine, F., Uddin, S., Sassano, A., Parmar, S., Brachmann, S.M., Majchrzak, B., Sonenberg, N., Hay, N., Fish, E.N., and Platanias, L.C. (2003). Activation of the p70 S6 kinase and phosphorylation of the 4E-BP1 repressor of mRNA translation by type I interferons. J Biol Chem 278, 27772-27780.

Lemmon, M.A., and Schlessinger, J. (2010). Cell signaling by receptor tyrosine kinases. Cell 141, 1117-1134. 
Leng, Y., Zhang, J., Badour, K., Arpaia, E., Freeman, S., Cheung, P., Siu, M., and Siminovitch, K. (2005). Abelsoninteractor-1 promotes WAVE2 membrane translocation and Abelson-mediated tyrosine phosphorylation required for WAVE2 activation. Proc Natl Acad Sci USA 102, 1098-1103.

Li, H., and Durbin, R. (2009). Fast and accurate short read alignment with Burrows-Wheeler transform. Bioinformatics 25, 1754-1760.

Li, Q.-R., Ning, Z.-B., Tang, J.-S., Nie, S., and Zeng, R. (2009). Effect of peptide-to-TiO 2 beads ratio on phosphopeptide enrichment selectivity. Journal of Proteome Research 8, 5375-5381.

Li, Z., Van Calcar, S., Qu, C., Cavenee, W.K., Zhang, M.Q., and Ren, B. (2003). A global transcriptional regulatory role for c-Myc in Burkitt's lymphoma cells. Proc Natl Acad Sci USA 100, 8164-8169.

Lim, W.A., and Pawson, T. (2010). Phosphotyrosine signaling: evolving a new cellular communication system. Cell 142, 661-667.

Lin, C.Y., Lovén, J., Rahl, P.B., Paranal, R.M., Burge, C.B., Bradner, J.E., Lee, T.I., and Young, R.A. (2012). Transcriptional amplification in tumor cells with elevated c-Myc. Cell 151, 56-67.

Liu, H., Sadygov, R.G., and Yates, J.R. (2004). A model for random sampling and estimation of relative protein abundance in shotgun proteomics. Analytical Chemistry 76, 4193-4201.

Love, C., Sun, Z., Jima, D., Li, G., Zhang, J., Miles, R., Richards, K.L., Dunphy, C.H., Choi, W.W.L., Srivastava, G., et al. (2012). The genetic landscape of mutations in Burkitt lymphoma. Nature Genetics 44, 1321-1325.

Lovén, J., Hoke, H.A., Lin, C.Y., Lau, A., Orlando, D.A., Vakoc, C.R., Bradner, J.E., Lee, T.I., and Young, R.A. (2013). Selective inhibition of tumor oncogenes by disruption of super-enhancers. Cell 153, 320-334.

Lowe, S.W., Cepero, E., and Evan, G. (2004). Intrinsic tumour suppression. Nature 432, 307-315.

Lugassy, J., Corso, J., Beach, D., Petrik, T., and Oellerich, T. (2015). Modulation of TCR responsiveness by the Grb2family adaptor, Gads. Cellular Signalling.

Lössner, C., Warnken, U., Pscherer, A., and Schnölzer, M. (2011). Preventing arginine-to-proline conversion in a cell-line-independent manner during cell cultivation under stable isotope labeling by amino acids in cell culture (SILAC) conditions. Analytical Biochemistry 412, 123-125.

MONTALVO, L., DOWLER, S., MONTALVO, L., CANTRELL, D., CANTRELL, D., Morrice, N., Morrice, N., Alessi, D.R., and Alessi, D.R. (2000). Phosphoinositide 3-kinase-dependent phosphorylation of the dual adaptor for phosphotyrosine and 3-phosphoinositides by the Src family of tyrosine kinase. Biochem J 349, 605-610.

MacNicol, M.C., Muslin, A.J., and MacNicol, A.M. (2000). Disruption of the 14-3-3 binding site within the B-Raf kinase domain uncouples catalytic activity from PC12 cell differentiation. J Biol Chem 275, 3803-3809.

Macek, B., Mann, M., and Olsen, J.V. (2008). Global and site-specific quantitative phosphoproteomics: principles and applications. Annu Rev Pharmacol Toxicol 49, 199-221.

Macian, F. (2005). NFAT proteins: key regulators of T-cell development and function. Nature Reviews Immunology 5, 472-484.

Makarov, A. (2000). Electrostatic axially harmonic orbital trapping: a high-performance technique of mass analysis. Analytical Chemistry 72, 1156-1162.

Makarov, A., Denisov, E., and Lange, O. (2009). Performance evaluation of a high-field Orbitrap mass analyzer. Journal of the American Society for Mass Spectrometry 20, 1391-1396.

Mallick, P., and Kuster, B. (2010). Proteomics: a pragmatic perspective. Nature Biotechnology 28, 695-709.

Mann, M., Ong, S.E., Grønborg, M., and Steen, H. (2002). Analysis of protein phosphorylation using mass spectrometry: deciphering the phosphoproteome. Trends in ....

Mann, M., and Jensen, O.N. (2003). Proteomic analysis of post-translational modifications. Nature Biotechnology $21,255$.

Manning, G., Whyte, D.B., Martinez, R., Hunter, T., and Sudarsanam, S. (2002). The protein kinase complement of the human genome. Science 298, 1912-1934. 
Marie-Cardine, A., Kirchgessner, H., Bruyns, E., Shevchenko, A., Mann, M., Autschbach, F., Ratnofsky, S., Meuer, S., and Schraven, B. (1999). SHP2-interacting transmembrane adaptor protein (SIT), a novel disulfide-linked dimer regulating human T cell activation. J Exp Med 189, 1181-1194.

Marshall, A.J., Niiro, H., Lerner, C.G., Yun, T.J., Thomas, S., Disteche, C.M., and Clark, E.A. (2000). A novel B lymphocyte-associated adaptor protein, Bam32, regulates antigen receptor signaling downstream of phosphatidylinositol 3-kinase. J Exp Med 191, 1319-1332.

Maruoka, M., Suzuki, J., Kawata, S., Yoshida, K., Hirao, N., Sato, S., Goff, S.P., Takeya, T., Tani, K., and Shishido, T. (2005). Identification of B cell adaptor for PI3-kinase (BCAP) as an Abl interactor 1-regulated substrate of Abl kinases. FEBS Letters 579, 2986-2990.

Marx, H., Marx, H., Lemeer, S., Lemeer, S., Schliep, J.E., Schliep, J.E., Matheron, L., Matheron, L., Mohammed, S., Mohammed, S., et al. (2013). A large synthetic peptide and phosphopeptide reference library for mass spectrometry-based proteomics. Nature Biotechnology 31, 557-564.

Matthews, H.R. (1995). Protein kinases and phosphatases that act on histidine, lysine, or arginine residues in eukaryotic proteins: a possible regulator of the mitogen-activated protein kinase cascade. Pharmacology \& Therapeutics 67, 323-350.

Matys, V., Kel-Margoulis, O.V., Fricke, E., Liebich, I., Land, S., Barre-Dirrie, A., Reuter, I., Chekmenev, D., Krull, M., Hornischer, K., et al. (2005). TRANSFAC and its module TRANSCompel: transcriptional gene regulation in eukaryotes. Nucleic Acids Res 34, D108-10.

Meins, M., Jenö, P., Müller, D., Richter, W.J., Rosenbusch, J.P., and Erni, B. (1993). Cysteine phosphorylation of the glucose transporter of Escherichia coli. J Biol Chem 268, 11604-11609.

Merienne, K., Jacquot, S., Zeniou, M., Pannetier, S., Sassone-Corsi, P., and Hanauer, A. (2000). Activation of RSK by UV-light: phosphorylation dynamics and involvement of the MAPK pathway. Oncogene 19, 4221-4229.

Mertins, P., Qiao, J.W., Patel, J., Udeshi, N.D., Clauser, K.R., Mani, D.R., Burgess, M.W., Gillette, M.A., Jaffe, J.D., and Carr, S.A. (2013). Integrated proteomic analysis of post-translational modifications by serial enrichment. Nature Methods 10, 634-637.

Michalski, A., Damoc, E., Hauschild, J.-P., Lange, O., Wieghaus, A., Makarov, A., Nagaraj, N., Cox, J., Mann, M., and Horning, S. (2011). Mass spectrometry-based proteomics using Q Exactive, a high-performance benchtop quadrupole Orbitrap mass spectrometer. Mol Cell Proteomics 10, M111.011015.

Michalski, A., Neuhauser, N., Cox, J., and Mann, M. (2012). A systematic investigation into the nature of tryptic HCD spectra. Journal of Proteome Research 11, 5479-5491.

Molyneux, E.M., Rochford, R., Griffin, B., Newton, R., Jackson, G., Menon, G., Harrison, C.J., Israels, T., and Bailey, S. (2012). Burkitt's lymphoma. Lancet 379, 1234-1244.

Monroe, J.G. (2006). ITAM-mediated tonic signalling through pre-BCR and BCR complexes. Nature Reviews Immunology 6, 283-294.

Murphy, K. (2011). Janeway's Immunobiology, 8th Edtion (Garland Science).

Muzio, M., Scielzo, C., Frenquelli, M., Bachi, A., De Palma, M., Alessio, M., Ghia, P., and Caligaris-Cappio, F. (2007). HS1 complexes with cytoskeleton adapters in normal and malignant chronic lymphocytic leukemia B cells. Leukemia 21, 2067-2070.

Myung, P.S., Derimanov, G.S., Jordan, M.S., Punt, J.A., and Liu, Q.H. (2001). Differential requirement for SLP-76 domains in T cell development and function. Immunity.

Mócsai, A., Ruland, J., and Tybulewicz, V.L.J. (2010). The SYK tyrosine kinase: a crucial player in diverse biological functions. Nature Reviews Immunology 10, 387-402.

NOSSAL, G.J., and LEDERBERG, J. (1958). Antibody production by single cells. Nature 181, 1419-1420.

Nagar, B., Bornmann, W.G., Pellicena, P., Schindler, T., Veach, D.R., Miller, W.T., Clarkson, B., and Kuriyan, J. (2002). Crystal structures of the kinase domain of c-Abl in complex with the small molecule inhibitors PD173955 and imatinib (STI-571). Cancer Research 62, 4236-4243.

Nagaraj, N., D'Souza, R.C.J., Cox, J., Olsen, J.V., and Mann, M. (2010). Feasibility of large-scale phosphoproteomics with higher energy collisional dissociation fragmentation. Journal of Proteome Research 9(12), 6786-6794. 
Nakamura, N., Nakamine, H., Tamaru, J.-I., Nakamura, S., Yoshino, T., Ohshima, K., and Abe, M. (2002). The distinction between Burkitt lymphoma and diffuse large B-Cell lymphoma with c-myc rearrangement. Mod Pathol $15,771-776$.

Nakao, M., Yokota, S., Iwai, T., Kaneko, H., Horiike, S., Kashima, K., Sonoda, Y., Fujimoto, T., and Misawa, S. (1996). Internal tandem duplication of the flt3 gene found in acute myeloid leukemia. Leukemia 10, 1911-1918.

Nakashima, T., Ishii, T., Tagaya, H., Seike, T., Nakagawa, H., Kanda, Y., Akinaga, S., Soga, S., and Shiotsu, Y. (2010). New molecular and biological mechanism of antitumor activities of KW-2478, a novel nonansamycin heat shock protein 90 inhibitor, in multiple myeloma cells. Clin Cancer Res 16, 2792-2802.

Neri, A., Barriga, F., Knowles, D.M., Magrath, I.T., and Dalla-Favera, R. (1988). Different regions of the immunoglobulin heavy-chain locus are involved in chromosomal translocations in distinct pathogenetic forms of Burkitt lymphoma. Proc Natl Acad Sci USA 85, 2748-2752.

The Cancer Genome Atlas Research Network (2013). Genomic and epigenomic landscapes of adult de novo acute myeloid leukemia. N Engl J Med 368, 2059-2074.

Neumann, K., Oellerich, T., Urlaub, H., and Wienands, J. (2009). The B-lymphoid Grb2 interaction code. Immunological Reviews 232, 135-149.

Nguyen, V., Cao, L., Lin, J.T., Hung, N., Ritz, A., Yu, K., Jianu, R., Ulin, S.P., Raphael, B.J., Laidlaw, D.H., et al. (2009). A new approach for quantitative phosphoproteomic dissection of signaling pathways applied to $T$ cell receptor activation. Mol Cell Proteomics 8, 2418-2431.

Nie, Z., Nie, Z., Hu, G., Hu, G., Wei, G., Wei, G., Cui, K., Cui, K., Yamane, A., Yamane, A., et al. (2012). c-Myc is a universal amplifier of expressed genes in lymphocytes and embryonic stem cells. Cell 151, 68-79.

Nielsen, M.L., Vermeulen, M., Bonaldi, T., Cox, J., Moroder, L., and Mann, M. (2008). lodoacetamide-induced artifact mimics ubiquitination in mass spectrometry. Nature Methods 5, 459-460.

Niiro, H., and Clark, E.A. (2002). Regulation of B-cell fate by antigen-receptor signals. Nature Reviews Immunology.

Nikolov, M., Schmidt, C., and Urlaub, H. (2012). Quantitative mass spectrometry-based proteomics: an overview. Methods Mol Biol 893, 85-100.

Nilsson, C.L. (2011). Advances in quantitative phosphoproteomics. Analytical Chemistry 84, 735-746.

Nilsson, J.A., and Cleveland, J.L. (2003). Myc pathways provoking cell suicide and cancer. Oncogene 22, 90079021.

Niu, H., Ye, B.H., and Dalla-Favera, R. (1998). Antigen receptor signaling induces MAP kinase-mediated phosphorylation and degradation of the BCL-6 transcription factor. Genes \& Development 12, 1953-1961.

Norton, A.J., and Isaacson, P.G. (1987). Detailed phenotypic analysis of B-cell lymphoma using a panel of antibodies reactive in routinely fixed wax-embedded tissue. The American Journal of Pathology 128, 225-240.

O'Conor, G.T., and DAVIES, J.N. (1960). Malignant tumors in African children. With special reference to malignant lymphoma. The Journal of Pediatrics 56, 526-535.

Oellerich, T., Oellerich, M.F., Engelke, M., and Münch, S. (2013). $\beta 2$ integrin-derived signals induce cell survival and proliferation of AML blasts by activating a Syk/STAT signaling axis. Blood.

Oellerich, T., Bremes, V., Neumann, K., Bohnenberger, H., Dittmann, K., Hsiao, H.-H., Engelke, M., Schnyder, T., Batista, F.D., Urlaub, H., et al. (2011). The B-cell antigen receptor signals through a preformed transducer module of SLP65 and CIN85. The EMBO Journal 30, 3620-3634.

Oellerich, T., Grønborg, M., Neumann, K., Hsiao, H.-H., Urlaub, H., and Wienands, J. (2009). SLP-65 phosphorylation dynamics reveals a functional basis for signal integration by receptor-proximal adaptor proteins. Mol Cell Proteomics 8, 1738-1750.

Oellerich, T., Mohr, S., Corso, J., Beck, J., Döbele, C., Braun, H., Cremer, A., Münch, S., Wicht, J., Oellerich, M.F., et al. (2015). FLT3-ITD and TLR9 use Bruton tyrosine kinase to activate distinct transcriptional programs mediating AML cell survival and proliferation. Blood 125, 1936-1947. 
Ohren, J.F., Chen, H., Pavlovsky, A., Whitehead, C., Zhang, E., Kuffa, P., Yan, C., McConnell, P., Spessard, C., Banotai, C., et al. (2004). Structures of human MAP kinase kinase 1 (MEK1) and MEK2 describe novel noncompetitive kinase inhibition. Nature structural \& molecular biology, 11, 1192-1197.

Olsen, J.V., and Mann, M. (2013). Status of large-scale analysis of post-translational modifications by mass spectrometry. Mol Cell Proteomics 12, 3444-3452.

Olsen, J.V., Blagoev, B., Gnad, F., Macek, B., Kumar, C., Mortensen, P., and Mann, M. (2006). Global, in vivo, and site-specific phosphorylation dynamics in signaling networks. Cell 127, 635-648.

Olsen, J.V., Macek, B., Lange, O., Makarov, A., Horning, S., and Mann, M. (2007). Higher-energy C-trap dissociation for peptide modification analysis. Nature Methods 4, 709-712.

Olsen, J.V., Ong, S.-E., and Mann, M. (2004). Trypsin cleaves exclusively C-terminal to arginine and lysine residues. Mol Cell Proteomics 3, 608-614.

Olsen, J.V., Schwartz, J.C., Griep-Raming, J., Nielsen, M.L., Damoc, E., Denisov, E., Lange, O., Remes, P., Taylor, D., Splendore, M., et al. (2009). A dual pressure linear ion trap Orbitrap instrument with very high sequencing speed. Mol Cell Proteomics 8, 2759-2769.

Olsen, J.V., Vermeulen, M., Santamaria, A., Kumar, C., Miller, M.L., Jensen, L.J., Gnad, F., Cox, J., Jensen, T.S., Nigg, E.A., et al. (2010). Quantitative phosphoproteomics reveals widespread full phosphorylation site occupancy during mitosis. Science Signaling 3, ra3.

Olsen, J.V., de Godoy, L.M.F., Li, G., Macek, B., Mortensen, P., Pesch, R., Makarov, A., Lange, O., Horning, S., and Mann, M. (2005). Parts per million mass accuracy on an Orbitrap mass spectrometer via lock mass injection into a C-trap. Mol Cell Proteomics 4, 2010-2021.

Ong, S.-E., and Mann, M. (2006). Mass spectrometry-based proteomics turns quantitative. Nature Chemical Biology 1, 252-262.

Ong, S.-E., and Mann, M. (2007). A practical recipe for stable isotope labeling by amino acids in cell culture (SILAC). Nature Protocols 1, 2650-2660.

Ong, S.-E., Blagoev, B., Kratchmarova, I., Kristensen, D.B., Steen, H., Pandey, A., and Mann, M. (2002). Stable isotope labeling by amino acids in cell culture, SILAC, as a simple and accurate approach to expression proteomics. Mol Cell Proteomics 1, 376-386.

Ong, S.-E., Kratchmarova, I., and Mann, M. (2003). Properties of 13C-substituted arginine in stable isotope labeling by amino acids in cell culture (SILAC). Journal of Proteome Research 2, 173-181.

Otero, D.C., Omori, S.A., and Rickert, R.C. (2000). Cd19-dependent activation of Akt kinase in B-lymphocytes. J Biol Chem 276, 1474-1478.

Pan, L., Wang, L., Hsu, C.-C., Zhang, J., lliuk, A., and Tao, W.A. (2015). Sensitive measurement of total protein phosphorylation level in complex protein samples. Analyst 140, 3390-3396.

Park, P.J. (2009). ChIP-seq: advantages and challenges of a maturing technology. Nature Reviews Genetics 10 , 669-680.

Park, S.K., Liao, L., Kim, J.Y., and Yates, J.R. (2009). A computational approach to correct arginine-to-proline conversion in quantitative proteomics. Nature Methods 6, 184-185.

Patrussi, L., Savino, M.T., Pellegrini, M., Paccani, S.R., Migliaccio, E., Plyte, S., Lanfrancone, L., Pelicci, P.G., and Baldari, C.T. (2005). Cooperation and selectivity of the two Grb2 binding sites of p52Shc in T-cell antigen receptor signaling to Ras family GTPases and Myc-dependent survival. Oncogene 24, 2218-2228.

Pellizzoni, L. (2007). Chaperoning ribonucleoprotein biogenesis in health and disease. EMBO Reports 8, 340-345.

Penas, E.M.M., Schilling, G., Behrmann, P., Klokow, M., Vettorazzi, E., Bokemeyer, C., and Dierlamm, J. (2014). Comprehensive cytogenetic and molecular cytogenetic analysis of 44 Burkitt lymphoma cell lines: secondary chromosomal changes characterization, karyotypic evolution, and comparison with primary samples. Genes Chromosomes Cancer 53, 497-515.

Peng, J., Schwartz, D., Elias, J.E., Thoreen, C.C., Cheng, D., Marsischky, G., Roelofs, J., Finley, D., and Gygi, S.P. (2003). A proteomics approach to understanding protein ubiquitination. Nature Biotechnology 21, 921-926. 
Polevoda, B., and Sherman, F. (2000). Na-terminal Acetylation of Eukaryotic Proteins. Journal of Biological Chemistry 275, 36479.

Ponader, S., Chen, S.-S., Buggy, J.J., Balakrishnan, K., Gandhi, V., Wierda, W.G., Keating, M.J., O'Brien, S., Chiorazzi, N., and Burger, J.A. (2011). The Bruton tyrosine kinase inhibitor PCI-32765 thwarts chronic lymphocytic leukemia cell survival and tissue homing in vitro and in vivo. Blood 119, 1182-1189.

Porras-Yakushi, T.R., Sweredoski, M.J., and Hess, S. (2015). ETD Outperforms CID and HCD in the Analysis of the Ubiquitylated Proteome. Journal of the American Society for Mass Spectrometry 26, 1580-1587.

Poulikakos, P.I., Zhang, C., Bollag, G., Shokat, K.M., and Rosen, N. (2010). RAF inhibitors transactivate RAF dimers and ERK signalling in cells with wild-type BRAF. Nature 464, 427-430.

Pruitt, K.D., Brown, G.R., Hiatt, S.M., Thibaud-Nissen, F., Astashyn, A., Ermolaeva, O., Farrell, C.M., Hart, J., Landrum, M.J., McGarvey, K.M., et al. (2013). RefSeq: an update on mammalian reference sequences. Nucleic Acids Res 42, D756-63.

Pucharcos, C., Estivill, X., and la Luna, de, S. (2000). Intersectin 2, a new multimodular protein involved in clathrinmediated endocytosis. FEBS Letters $478,43$.

Pérez-Benavente, B., García, J.L., Rodríguez, M.S., Pineda-Lucena, A., Piechaczyk, M., de Mora, J.F., and Farràs, R. (2012). GSK3-SCFFBXW7 targets JunB for degradation in G2 to preserve chromatid cohesion before anaphase. Oncogene 32, 2189.

Quentmeier, H., Reinhardt, J., Zaborski, M., and Drexler, H.G. (2003). FLT3 mutations in acute myeloid leukemia cell lines. Leukemia 17, 120-124.

R Core Team (2014). R: a language and environment for statistical computing. Vienna, Austria: R Foundation for Statistical Computing; 2012.

Raaijmakers, L.M., Giansanti, P., Possik, P.A., Mueller, J., Peeper, D.S., Heck, A.J.R., and Altelaar, A.F.M. (2015). PhosphoPath: Visualization of Phosphosite-centric Dynamics in Temporal Molecular Networks. Journal of Proteome Research 14, 4332-4341.

Rebollo, A., Ayllón, V., Fleischer, A., Martínez, C.A., and Zaballos, A. (2001). The association of Aiolos transcription factor and $\mathrm{BCl}-\mathrm{xL}$ is involved in the control of apoptosis. J Immunol 167, 6366-6373.

Refaeli, Y., Young, R.M., Turner, B.C., Duda, J., Field, K.A., and Bishop, J.M. (2008). The B cell antigen receptor and overexpression of MYC can cooperate in the genesis of B cell lymphomas. PLoS Biology 6, e152.

Reth, M. (1989). Antigen receptor tail clue. Nature 338, 383-384.

Reth, M., and Wienands, J. (1997). Initiation and processing of signals from the B cell antigen receptor. Annual Review of ... 15, 453-479.

Rickert, R.C. (2013). New insights into pre-BCR and BCR signalling with relevance to B cell malignancies. Nature Reviews Immunology 13, 578-591.

Rizzieri, D.A., Johnson, J.L., Byrd, J.C., Lozanski, G., Blum, K.A., Powell, B.L., Shea, T.C., Nattam, S., Hoke, E., Cheson, B.D., et al. (2014). Improved efficacy using rituximab and brief duration, high intensity chemotherapy with filgrastim support for Burkitt or aggressive lymphomas: cancer and Leukemia Group B study 10002 . Br J Haematol 165, 102-111.

Rizzieri, D.A., Johnson, J.L., Niedzwiecki, D., Lee, E.J., Vardiman, J.W., Powell, B.L., Barcos, M., Bloomfield, C.D., Schiffer, C.A., Peterson, B.A., et al. (2004). Intensive chemotherapy with and without cranial radiation for Burkitt leukemia and lymphoma. Cancer 100, 1438.

Roepstorff, P., and Fohlman, J. (1984). Proposal for a common nomenclature for sequence ions in mass spectra of peptides. Biomed Mass Spectrom 11, 601.

Rolland, D., Basrur, V., Conlon, K., Wolfe, T., Fermin, D., Nesvizhskii, A.I., Lim, M.S., and Elenitoba-Johnson, K.S.J. (2014). Global phosphoproteomic profiling reveals distinct signatures in B-cell non-Hodgkin lymphomas. The American Journal of Pathology 184, 1331-1342.

Romero, F., Martínez-A, C., Camonis, J., and Rebollo, A. (1999). Aiolos transcription factor controls cell death in T cells by regulating Bcl-2 expression and its cellular localization. The EMBO Journal 18, 3419-3430. 
Ross, P.L., Huang, Y.N., Marchese, J.N., Williamson, B., Parker, K., Hattan, S., Khainovski, N., Pillai, S., Dey, S., Daniels, S., et al. (2004). Multiplexed protein quantitation in Saccharomyces cerevisiae using amine-reactive isobaric tagging reagents. Mol Cell Proteomics 3, 1154-1169.

Rowland, S.L., Leahy, K.F., Halverson, R., Torres, R.M., and Pelanda, R. (2010). BAFF receptor signaling aids the differentiation of immature B cells into transitional B cells following tonic BCR signaling. J Immunol 185, 45704581.

Ruprecht, B., Zecha, J., Heinzlmeir, S., Médard, G., Lemeer, S., and Kuster, B. (2015). Evaluation of Kinase Activity Profiling Using Chemical Proteomics. ACS Chemical Biology 10, 2743-2752.

Rush, J., Moritz, A., Lee, K.A., Guo, A., Goss, V.L., Spek, E.J., Zhang, H., Zha, X.-M., Polakiewicz, R.D., and Comb, M.J. (2004). Immunoaffinity profiling of tyrosine phosphorylation in cancer cells. Nature Biotechnology 23, 94101.

Rushworth, S.A., Murray, M.Y., Zaitseva, L., Bowles, K.M., and MacEwan, D.J. (2013). Identification of Bruton's tyrosine kinase as a therapeutic target in acute myeloid leukemia. Blood 123, 1229-1238.

Sako, T., Tauber, A.I., Jeng, A.Y., Yuspa, S.H., and Blumberg, P.M. (1988). Contrasting actions of staurosporine, a protein kinase $C$ inhibitor, on human neutrophils and primary mouse epidermal cells. Cancer Research $48,4646-$ 4650 .

Samelson, L.E. (2011). Immunoreceptor signaling. Cold Spring Harbor Perspectives in Biology 3, a011510.

Santoli, D., Yang, Y.C., Clark, S.C., Kreider, B.L., Caracciolo, D., and Rovera, G. (1987). Synergistic and antagonistic effects of recombinant human interleukin (IL) 3, IL-1 alpha, granulocyte and macrophage colony-stimulating factors (G-CSF and M-CSF) on the growth of GM-CSF-dependent leukemic cell lines. J Immunol 139, 3348-3354.

Sargin, B., Choudhary, C., Crosetto, N., Schmidt, M.H.H., Grundler, R., Rensinghoff, M., Thiessen, C., Tickenbrock, L., Schwäble, J., Brandts, C., et al. (2007). Flt3-dependent transformation by inactivating c-Cbl mutations in AML. Blood 110, 1004-1012.

Satpathy, S., Wagner, S.A., Beli, P., Gupta, R., Kristiansen, T.A., Malinova, D., Francavilla, C., Tolar, P., Bishop, G.A., Hostager, B.S., et al. (2015). Systems-wide analysis of BCR signalosomes and downstream phosphorylation and ubiquitylation. Molecular Systems Biology 11, 810.

Savino, M., Annibali, D., Carucci, N., Favuzzi, E., and Cole, M.D. (2011). The action mechanism of the Myc inhibitor termed Omomyc may give clues on how to target Myc for cancer therapy. PLoS ONE.

Savitski, M.M., Lemeer, S., Boesche, M., Lang, M., Mathieson, T., Bantscheff, M., and Kuster, B. (2010). Confident phosphorylation site localization using the Mascot Delta Score. Mol Cell Proteomics 10, M110.003830.

Scheltema, R.A., Hauschild, J.-P., Lange, O., Hornburg, D., Denisov, E., Damoc, E., Kuehn, A., Makarov, A., and Mann, M. (2014). The Q Exactive HF, a Benchtop mass spectrometer with a pre-filter, high-performance quadrupole and an ultra-high-field Orbitrap analyzer. Mol Cell Proteomics 13, 3698-3708.

Schindler, T., Bornmann, W., Pellicena, P., Miller, W.T., Clarkson, B., and Kuriyan, J. (2000). Structural mechanism for STI-571 inhibition of abelson tyrosine kinase. Science 289, 1938-1942.

Schlessinger, J., and Lemmon, M.A. (2003). SH2 and PTB domains in tyrosine kinase signaling. Sci STKE 2003, RE12.

Schmidt, A., Karas, M., and Dülcks, T. (2003). Effect of different solution flow rates on analyte ion signals in nanoESI MS, or: when does ESI turn into nano-ESI? Journal of the American Society for Mass Spectrometry 14, 492.

Schmitz, R., Ceribelli, M., Pittaluga, S., Wright, G., and Staudt, L.M. (2014). Oncogenic mechanisms in Burkitt lymphoma. Cold Spring Harb Perspect Med 4, a014282.

Schmitz, R., Young, R.M., Ceribelli, M., Jhavar, S., Xiao, W., Zhang, M., Wright, G., Shaffer, A.L., Hodson, D.J., Buras, E., et al. (2012). Burkitt lymphoma pathogenesis and therapeutic targets from structural and functional genomics. Nature 490, 116-120.

Schroeder, M.J., Shabanowitz, J., and Schwartz, J.C. (2004). A neutral loss activation method for improved phosphopeptide sequence analysis by quadrupole ion trap mass spectrometry. Analytical Chemistry 76, 3590.

Schwanhäusser, B., Busse, D., Li, N., Dittmar, G., Schuchhardt, J., Wolf, J., Chen, W., and Selbach, M. (2011). Global quantification of mammalian gene expression control. Nature 473, 337-342. 
Schwartz, D., and Gygi, S.P. (2005). An iterative statistical approach to the identification of protein phosphorylation motifs from large-scale data sets. Nature Biotechnology 23, 1391-1398.

Schwartz, R.S., Lenz, G., and Staudt, L.M. (2010). Aggressive Lymphomas. New England Journal of Medicine 362, 1417.

Schwartz-Albiez, R., Dörken, B., Hofmann, W., and Moldenhauer, G. (1988). The B cell-associated CD37 antigen (gp40-52). Structure and subcellular expression of an extensively glycosylated glycoprotein. J Immunol 140, 905914.

Schweigel, H., Geiger, J., Beck, F., Buhs, S., Gerull, H., Walter, U., Sickmann, A., and Nollau, P. (2013). Deciphering of ADP-induced, phosphotyrosine-dependent signaling networks in human platelets by Src-homology 2 region (SH2)-profiling. Proteomics 13, 1016-1027.

Scigelova, M., and Makarov, A. (2006). Orbitrap mass analyzer--overview and applications in proteomics. Proteomics 6 Suppl 2, 16-21.

Sefton, B.M., Hunter, T., Beemon, K., and Eckhart, W. (1980). Evidence that the phosphorylation of tyrosine is essential for cellular transformation by Rous sarcoma virus. Cell 20, 807-816.

Sela, M., Bogin, Y., Beach, D., Oellerich, T., Lehne, J., Smith-Garvin, J.E., Okumura, M., Starosvetsky, E., Kosoff, R., Libman, E., et al. (2011). Sequential phosphorylation of SLP-76 at tyrosine 173 is required for activation of T and mast cells. The EMBO Journal 30, 3160-3172.

Seitz, V., Butzhammer, P., Hirsch, B., Hecht, J., Gütgemann, I., Ehlers, A., Lenze, D., Oker, E., Sommerfeld, A., Wall, von der, E., et al. (2011). Deep sequencing of MYC DNA-binding sites in Burkitt lymphoma. PLoS ONE 6, e26837.

Senko, M.W., Beu, S.C., and McLaffertycor, F.W. (1995). Determination of monoisotopic masses and ion populations for large biomolecules from resolved isotopic distributions. Journal of the American Society for Mass Spectrometry 6, 229.

Shaffer, A.L., and Staudt, L.M. (2012). The case of the missing C-Myc. Nature Immunology 13, 1029-1031.

Shaffer, A.L., Young, R.M., and Staudt, L.M. (2012). Pathogenesis of human B cell lymphomas. Annual Review of Immunology 30, 565-610.

Shalom-Barak, T., and Knaus, U.G. (2002). A p21-activated kinase-controlled metabolic switch up-regulates phagocyte NADPH oxidase. J Biol Chem 277, 40659-40665.

Shannon, P., Markiel, A., Ozier, O., Baliga, N.S., Wang, J.T., Ramage, D., Amin, N., Schwikowski, B., and Ideker, T. (2003). Cytoscape: a software environment for integrated models of biomolecular interaction networks. Genome Res 13, 2498-2504.

Shapiro, G.I., Kwak, E., Shapiro, G.I., Dezube, B.J., Yule, M., Ayrton, J., Kwak, E., Lyons, J., Dezube, B.J., Mahadevan, D., et al. (2014). First-in-human phase I dose escalation study of a second-generation non-ansamycin HSP90 inhibitor, AT13387, in patients with advanced solid tumors. Clin Cancer Res 21, 87-97.

Sharma, K., D'Souza, R.C.J., Tyanova, S., Schaab, C., Wiśniewski, J.R., Cox, J., and Mann, M. (2014). Ultradeep human phosphoproteome reveals a distinct regulatory nature of Tyr and Ser/Thr-based signaling. Cell Reports 8 , 1583-1594.

Shevchenko, A., Tomas, H., Havlis, J., Olsen, J.V., and Mann, M. (2007). In-gel digestion for mass spectrometric characterization of proteins and proteomes. Nature Protocols 1, 2856-2860.

Silver, K., and Cornall, R.J. (2003). Isotype control of B cell signaling. Sci STKE 2003, pe21.

Simon, J.A., and Kingston, R.E. (2013). Occupying chromatin: Polycomb mechanisms for getting to genomic targets, stopping transcriptional traffic, and staying put. Molecular Cell 49, 808-824.

Smith, J.L., Jones, D.B., Bell, A.J., and Wright, D.H. (1989). Correlation between histology and immunophenotype in a series of 322 cases of non-Hodgkin's lymphoma. Hematological ... 7, 37.

Soucek, L., Jucker, R., Panacchia, L., Ricordy, R., Tatò, F., and Nasi, S. (2002). Omomyc, a potential Myc dominant negative, enhances Myc-induced apoptosis. Cancer Research 62, 3507-3510.

Spender, L.C., and Inman, G.J. (2014). Developments in Burkitt's lymphoma: novel cooperations in oncogenic MYC signaling. Cancer Management and Research 6, 27-38. 
Spurgeon, S.E., Coffey, G., Fletcher, L.B., Burke, R., Tyner, J.W., Druker, B.J., Betz, A., DeGuzman, F., Pak, Y., Baker, D., et al. (2012). The selective SYK inhibitor P505-15 (PRT062607) inhibits B cell signaling and function in vitro and in vivo and augments the activity of fludarabine in chronic lymphocytic leukemia. J Pharmacol Exp Ther 344, 378-387.

Srinivasan, L., Sasaki, Y., Calado, D.P., Zhang, B., Paik, J.H., DePinho, R.A., Kutok, J.L., Kearney, J.F., Otipoby, K.L., and Rajewsky, K. (2009). PI3 kinase signals BCR-dependent mature B cell survival. Cell 139, 573-586.

Stadanlick, J.E., Kaileh, M., Karnell, F.G., Scholz, J.L., Miller, J.P., Quinn, W.J., Brezski, R.J., Treml, L.S., Jordan, K.A., Monroe, J.G., et al. (2008). Tonic B cell antigen receptor signals supply an NF-kappaB substrate for prosurvival BLyS signaling. Nature Immunology 9, 1379-1387.

Steen, H., Kuster, B., Fernandez, M., Pandey, A., and Mann, M. (2001). Detection of tyrosine phosphorylated peptides by precursor ion scanning quadrupole TOF mass spectrometry in positive ion mode. Analytical Chemistry 73, 1440-1448.

Steen, H., and Mann, M. (2004). The ABC's (and XYZ's) of peptide sequencing. Nature Reviews Molecular Cell Biology 5, 699-711.

Stradal, T., Courtney, K.D., Rottner, K., Hahne, P., Small, J.V., and Pendergast, A.M. (2001). The Abl interactor proteins localize to sites of actin polymerization at the tips of lamellipodia and filopodia. Curr Biol 11, 891-895.

Strahl, B.D., and Allis, C.D. (2000). The language of covalent histone modifications. Nature.

Sturm, M., Bertsch, A., Gröpl, C., Hildebrandt, A., Hussong, R., Lange, E., Pfeifer, N., Schulz-Trieglaff, O., Zerck, A., Reinert, K., et al. (2008). OpenMS - an open-source software framework for mass spectrometry. BMC Bioinformatics 9, 163.

Sun, L., Zhu, G., and Dovichi, N.J. (2012). Comparison of the LTQ-Orbitrap Velos and the Q-Exactive for proteomic analysis of 1-1000 ng RAW 264.7 cell lysate digests. Rapid Commun Mass Spectrom 27, 157-162.

Sun, L., and Chen, Z.J. (2004). The novel functions of ubiquitination in signaling. Current Opinion in Cell Biology $16,119-126$.

Suthers, A.N., and Young, L.J. (2014). Isoforms of the CD79 signal transduction component of the macropod Bcell receptor. Developmental \& Comparative Immunology 47, 185-190.

Swaney, D.L., Beltrao, P., Starita, L., Guo, A., Rush, J., Fields, S., Krogan, N.J., and Villen, J. (2013). Global analysis of phosphorylation and ubiquitylation cross-talk in protein degradation. Nature Methods 10, 676-682.

Swaney, D.L., Wenger, C.D., Thomson, J.A., and Coon, J.J. (2009). Human embryonic stem cell phosphoproteome revealed by electron transfer dissociation tandem mass spectrometry. Proc Natl Acad Sci USA 106, 995-1000.

Swanson, B.J., Jäck, H.M., and Lyons, G.E. (1998). Characterization of myocyte enhancer factor 2 (MEF2) expression in B and T cells: MEF2C is a B cell-restricted transcription factor in lymphocytes. Molecular Immunology 35, 445-458.

Szklarczyk, D., Franceschini, A., Wyder, S., Forslund, K., Heller, D., Huerta-Cepas, J., Szklarczyk, D., Franceschini, A., Wyder, S., Forslund, K., et al. (2014). STRING v10: protein-protein interaction networks, integrated over the tree of life. Nucleic Acids Res 43, D447-52.

Taldone, T., Gozman, A., Maharaj, R., and Chiosis, G. (2008). Targeting Hsp90: small-molecule inhibitors and their clinical development. Curr Opin Pharmacol 8, 370-374.

Tanaka, K., Waki, H., Ido, Y., Akita, S., Yoshida, Y., Yoshida, T., and Matsuo, T. (1988). Protein and polymer analyses up tom/z 100000 by laser ionization time-of-flight mass spectrometry. Rapid Communications in Mass Spectrometry 2, 151.

Tangye, S.G., Nichols, K.E., Hare, N.J., and van de Weerdt, B.C.M. (2003). Functional requirements for interactions between CD84 and Src homology 2 domain-containing proteins and their contribution to human $\mathrm{T}$ cell activation. J Immunol 171, 2485-2495.

Tanis, K.Q., Veach, D., Duewel, H.S., Bornmann, W.G., and Koleske, A.J. (2003). Two distinct phosphorylation pathways have additive effects on Abl family kinase activation. Mol Cell Biol 23, 3884-3896.

Tarrant, J.M., Robb, L., van Spriel, A.B., and Wright, M.D. (2003). Tetraspanins: molecular organisers of the leukocyte surface. Trends in Immunology 24, 610-617. 
Taub, R., Kirsch, I., Morton, C., Lenoir, G., Swan, D., Tronick, S., Aaronson, S., and Leder, P. (1982). Translocation of the c-myc gene into the immunoglobulin heavy chain locus in human Burkitt lymphoma and murine plasmacytoma cells. Proc Natl Acad Sci USA 79, 7837-7841.

Taus, T., Köcher, T., Pichler, P., Paschke, C., Schmidt, A., Henrich, C., and Mechtler, K. (2011). Universal and confident phosphorylation site localization using phosphoRS. Journal of Proteome Research 10, 5354-5362.

Teckchandani, A.M., Panetti, T.S., and Tsygankov, A.Y. (2005). c-Cbl regulates migration of v-Abl-transformed NIH 3 T3 fibroblasts via Rac1. Experimental Cell Research 307, 247-258.

Thingholm, T.E., Jørgensen, T.J.D., Jensen, O.N., and Larsen, M.R. (2007). Highly selective enrichment of phosphorylated peptides using titanium dioxide. Nature Protocols 1, 1929-1935.

Thomas, D.D.H., Frey, C.L., Messenger, S.W., August, B.K., and Groblewski, G.E. (2010). A role for tumor protein TPD52 phosphorylation in endo-membrane trafficking during cytokinesis. Biochem Biophys Res Commun 402, 583-587.

Thomas, D.D.H., Martin, C.L., Weng, N., Byrne, J.A., and Groblewski, G.E. (2009). Tumor protein D52 expression and $\mathrm{Ca2+-dependent} \mathrm{phosphorylation} \mathrm{modulates} \mathrm{lysosomal} \mathrm{membrane} \mathrm{protein} \mathrm{trafficking} \mathrm{to} \mathrm{the} \mathrm{plasma}$ membrane. Am J Physiol, Cell Physiol 298, C725-39.

Thomson, B.A., and Iribarne, J.V. (1979). Field induced ion evaporation from liquid surfaces at atmospheric pressure. The Journal of Chemical Physics 71, 4451.

Thrower, J.S., Hoffman, L., Rechsteiner, M., and Pickart, C.M. (2000). Recognition of the polyubiquitin proteolytic signal. The EMBO Journal 19, 94-102.

Tourrière, H., Gallouzi, I., and Chebli, K. (2001). RasGAP-associated endoribonuclease G3Bp: selective RNA degradation and phosphorylation-dependent localization. Molecular and Cellular Biology, 21(22), 7747-7760.

Tran, N.H., Wu, X., and Frost, J.A. (2005). B-Raf and Raf-1 are regulated by distinct autoregulatory mechanisms. J Biol Chem 280, 16244-16253.

Trepel, J., Mollapour, M., Giaccone, G., and Neckers, L. (2010). Targeting the dynamic HSP90 complex in cancer. Nature Reviews Cancer 10, 537-549.

Tsai, C.-M., Wu, H.-Y., Su, T.-H., Kuo, C.-W., Huang, H.-W., Chung, C.-H., Chen, C.-S., Khoo, K.-H., Chen, Y.-J., and Lin, K.-I. (2014). Phosphoproteomic analyses reveal that galectin-1 augments the dynamics of B-cell receptor signaling. Journal of Proteomics 103, 241-253.

Tsang, E., Giannetti, A.M., Shaw, D., Dinh, M., Tse, J.K.Y., Gandhi, S., Ho, H., Wang, S., Papp, E., and Bradshaw, J.M. (2008). Molecular mechanism of the Syk activation switch. J Biol Chem 283, 32650-32659.

Uy, R., and Wold, F. (1977). Posttranslational covalent modification of proteins. Science 198, 890.

Valouev, A., Johnson, D.S., Sundquist, A., Medina, C., Anton, E., Batzoglou, S., Myers, R.M., and Sidow, A. (2009). Genome-wide analysis of transcription factor binding sites based on ChIP-Seq data. Nature Methods 5, 829-834.

Van Hoof, D., Pinkse, M.W.H., Oostwaard, D.W.-V., Mummery, C.L., Heck, A.J.R., and Krijgsveld, J. (2007). An experimental correction for arginine-to-proline conversion artifacts in SILAC-based quantitative proteomics. Nature Methods 4, 677-678.

Vasilescu, J., Smith, J.C., Ethier, M., and Figeys, D. (2005). Proteomic analysis of ubiquitinated proteins from human MCF-7 breast cancer cells by immunoaffinity purification and mass spectrometry. Journal of Proteome Research 4, 2192-2200.

Vijayan, V., Baumgart-Vogt, E., Naidu, S., Qian, G., and Immenschuh, S. (2011). Bruton's tyrosine kinase is required for TLR-dependent heme oxygenase-1 gene activation via Nrf2 in macrophages. J Immunol 187, 817827.

Villar-Garea, A., and Imhof, A. (2006). The analysis of histone modifications. Biochimica Et Biophysica Acta (BBA) - Proteins and Proteomics 1764, 1932.

Wagner, S.A., Beli, P., Weinert, B.T., Nielsen, M.L., Cox, J., Mann, M., and Choudhary, C. (2011). A proteomewide, quantitative survey of in vivo ubiquitylation sites reveals widespread regulatory roles. Mol Cell Proteomics 10, M111.013284. 
Wakabayashi, C., Adachi, T., Wienands, J., and Tsubata, T. (2002). A distinct signaling pathway used by the IgGcontaining B cell antigen receptor. Science 298, 2392-2395.

Walsh, C.T., Garneau-Tsodikova, S., and Gatto, G.J. (2005). Protein posttranslational modifications: the chemistry of proteome diversifications. Angew Chem Int Ed Engl 44, 7342-7372.

Wang, J.H., Avitahl, N., Cariappa, A., Friedrich, C., Ikeda, T., Renold, A., Andrikopoulos, K., Liang, L., Pillai, S., Morgan, B.A., et al. (1998). Aiolos regulates B cell activation and maturation to effector state. Immunity 9, 543553.

Wang, M.L., Rule, S., Martin, P., Goy, A., Auer, R., Kahl, B.S., Jurczak, W., Advani, R.H., Romaguera, J.E., Williams, M.E., et al. (2013). Targeting BTK with ibrutinib in relapsed or refractory mantle-cell lymphoma. N Engl J Med $369,507-516$.

Wang, Q., Zhang, Y., Yang, C., Xiong, H., Lin, Y., Yao, J., Li, H., Xie, L., Zhao, W., Yao, Y., et al. (2010). Acetylation of metabolic enzymes coordinates carbon source utilization and metabolic flux. Science 327, 1004-1007.

Wang, X., Li, J.-P., Chiu, L.-L., Lan, J.-L., Chen, D.-Y., Boomer, J., and Tan, T.-H. (2012). Attenuation of T cell receptor signaling by serine phosphorylation-mediated lysine 30 ubiquitination of SLP-76 protein. J Biol Chem 287, 3409134100.

Warnes, G.R., Bolker, B., Bonebakker, L., Gentleman, R., Huber, W., Liaw, A., Lumley, T., Maechler, M., Magnusson, A., Moeller, S., et al. (2013). Gplots: various R programming tools for plotting data, v2.12.1.

Washburn, M.P., Wolters, D., and Yates, J.R. (2001). Large-scale analysis of the yeast proteome by multidimensional protein identification technology. Nature Biotechnology 19, 242-247.

Weinert, B.T., Wagner, S.A., Horn, H., Henriksen, P., Liu, W.R., Olsen, J.V., Jensen, L.J., and Choudhary, C. (2011). Proteome-wide mapping of the Drosophila acetylome demonstrates a high degree of conservation of lysine acetylation. Science Signaling 4, ra48.

Weinstein, I.B., Begemann, M., Zhou, P., Han, E.K., Sgambato, A., Doki, Y., Arber, N., Ciaparrone, M., and Yamamoto, H. (1999). Disorders in cell circuitry associated with multistage carcinogenesis: exploitable targets for cancer prevention and therapy. Clin Cancer Res 3, 2696-2702.

Weinstein, I.B., and Joe, A.K. (2006). Mechanisms of disease: Oncogene addiction--a rationale for molecular targeting in cancer therapy. Nature Clinical Practice Oncology 3, 448-457.

Welcker, M., Orian, A., Jin, J., Grim, J.E., Harper, J.W., Eisenman, R.N., and Clurman, B.E. (2004). The Fbw7 tumor suppressor regulates glycogen synthase kinase 3 phosphorylation-dependent c-Myc protein degradation. Proceedings of the National Academy of Sciences 101, 9085.

White, R.G. (1958). Antibody Production by Single Cells. Nature 182, 1383.

Whitesell, L., and Lindquist, S.L. (2005). HSP90 and the chaperoning of cancer. Nature Reviews Cancer 5, 761772.

Whitfield, T.W., Wang, J., Collins, P.J., Partridge, E.C., Aldred, S.F., Trinklein, N.D., Myers, R.M., and Weng, Z. (2012). Functional analysis of transcription factor binding sites in human promoters. Genome Biol 13, R50.

Whitmarsh, A.J., and Davis, R.J. (2000). Regulation of transcription factor function by phosphorylation. Cellular and Molecular Life Sciences CMLS 57, 1172-1183.

Wienands, J., Schweikert, J., Wollscheid, B., Jumaa, H., Nielsen, P.J., and Reth, M. (1998). SLP-65: a new signaling component in $B$ lymphocytes which requires expression of the antigen receptor for phosphorylation. J Exp Med 188, 791-795.

Wilhelm, M., Schlegl, J., Hahne, H., and Gholami, A.M. (2014). Mass-spectrometry-based draft of the human proteome. Nature.

Wilkins, M.R., Sanchez, J.C., Gooley, A.A., Appel, R.D., Humphery-Smith, I., Hochstrasser, D.F., and Williams, K.L. (1996a). Progress with proteome projects: why all proteins expressed by a genome should be identified and how to do it. Biotechnol Genet Eng Rev 13, 19-50.

Wilkins, M.R., Pasquali, C., Appel, R.D., Ou, K., Golaz, O., Sanchez, J.-C., Yan, J.X., Gooley, A.A., Hughes, G., Humphery-Smith, I., et al. (1996b). From Proteins to Proteomes: Large Scale Protein Identification by TwoDimensional Electrophoresis and Arnino Acid Analysis. Bio/Technology 14, 61. 
Williams, T.E., Subramanian, S., Verhagen, J., McBride, C.M., Costales, A., Sung, L., Antonios-McCrea, W., McKenna, M., Louie, A.K., Ramurthy, S., et al. (2015). Discovery of RAF265: A Potent mut-B-RAF Inhibitor for the Treatment of Metastatic Melanoma. ACS Med Chem Lett 6, 961-965.

Witze, E.S., Old, W.M., Resing, K.A., and Ahn, N.G. (2007). Mapping protein post-translational modifications with mass spectrometry. Nature Methods 4, 798-806.

Wu, C.F., Liu, S., Lee, Y.C., Wang, R., Sun, S., Yin, F., Bornmann, W.G., Yu-Lee, L.-Y., Gallick, G.E., Zhang, W., et al. (2013). RSK promotes G2/M transition through activating phosphorylation of Cdc25A and Cdc25B. Oncogene 33, 2385-2394.

Xu, G., Paige, J.S., and Jaffrey, S.R. (2010). Global analysis of lysine ubiquitination by ubiquitin remnant immunoaffinity profiling. Nature Biotechnology $28,868-873$.

Yablonski, D., Kuhne, M.R., Kadlecek, T., and Weiss, A. (1998). Uncoupling of nonreceptor tyrosine kinases from PLC-gamma1 in an SLP-76-deficient T cell. Science 281, 413-416.

Yada, M., Hatakeyama, S., Kamura, T., Nishiyama, M., Tsunematsu, R., Imaki, H., Ishida, N., Okumura, F., Nakayama, K., and Nakayama, K.I. (2004). Phosphorylation-dependent degradation of c-Myc is mediated by the F-box protein Fbw7. The EMBO Journal 23, 2116-2125.

Yaffe, M.B., and Elia, A.E. (2001). Phosphoserine/threonine-binding domains. Current Opinion in Cell Biology 13, 131-138.

Yaffe, M.B. (2002). Phosphotyrosine-binding domains in signal transduction. Nature Reviews Molecular Cell Biology 3, 177-186.

Ye, Y., and Rape, M. (2009). Building ubiquitin chains: E2 enzymes at work. Nature Reviews Molecular Cell Biology $10,755-764$.

Young, R.M., and Staudt, L.M. (2013). Targeting pathological B cell receptor signalling in lymphoid malignancies. Nature Reviews Drug Discovery 12, 229-243.

Zaitseva, L., Murray, M.Y., Shafat, M.S., Lawes, M.J., MacEwan, D.J., Bowles, K.M., and Rushworth, S.A. (2014). Ibrutinib inhibits SDF1/CXCR4 mediated migration in AML. Oncotarget 5, 9930.

Zarei, M., Sprenger, A., Metzger, F., Gretzmeier, C., and Dengjel, J. (2011). Comparison of ERLIC-TiO2 , HILICTiO2, and SCX-TiO2 for Global Phosphoproteomics Approaches. Journal of Proteome Research 10, 3474.

Zeng, L., and Zhou, M.M. (2002). Bromodomain: an acetyl-lysine binding domain. FEBS Letters 513, 124-128.

Zhang, B.-H., and Guan, K.-L. (2001). REGULATION OF THE Raf KINASE BY PHOSPHORYLATION. Experimental Lung Research 27, 269.

Zhang, J., Yang, P.L., and Gray, N.S. (2008a). Targeting cancer with small molecule kinase inhibitors. Nature Reviews Cancer 9, 28-39.

Zhang, M.-Y., Pace, N., Kerns, E.H., Kleintop, T., Kagan, N., and Sakuma, T. (2005). Hybrid triple quadrupole-linear ion trap mass spectrometry in fragmentation mechanism studies: application to structure elucidation of buspirone and one of its metabolites. J Mass Spectrom 40, 1017-1029.

Zhang, Y., Wang, Z., Li, X., and Magnuson, N.S. (2008). Pim kinase-dependent inhibition of c-Myc degradation. Oncogene 27, 4809-4819.

Zhang, Y., Fonslow, B.R., Shan, B., Baek, M.-C., and Yates, J.R. (2013). Protein analysis by shotgun/bottom-up proteomics. Chemical Reviews 113, 2343-2394.

Zhao, S., Xu, W., Jiang, W., Yu, W., Lin, Y., Zhang, T., Yao, J., Zhou, L., Zeng, Y., Li, H., et al. (2010). Regulation of cellular metabolism by protein lysine acetylation. Science 327, 1000-1004.

Zhao, Y., and Jensen, O.N. (2009). Modification-specific proteomics: strategies for characterization of posttranslational modifications using enrichment techniques. Proteomics 9, 4632-4641.

Zhao, Z., Tan, Z., Diltz, C.D., You, M., and Fischer, E.H. (1996). Activation of mitogen-activated protein (MAP) kinase pathway by pervanadate, a potent inhibitor of tyrosine phosphatases. J Biol Chem 271, 22251-22255.

Zimmermann, S., and Moelling, K. (1999). Phosphorylation and regulation of Raf by Akt (protein kinase B). Science 286, 1741-1744. 
Zippo, A., De Robertis, A., Serafini, R., and Oliviero, S. (2007). PIM1-dependent phosphorylation of histone H3 at serine 10 is required for MYC-dependent transcriptional activation and oncogenic transformation. Nature Cell Biology 9, 932-944.

de Rooij, M.F.M., Kuil, A., Geest, C.R., Eldering, E., Chang, B.Y., Buggy, J.J., Pals, S.T., and Spaargaren, M. (2012). The clinically active BTK inhibitor PCl-32765 targets B-cell receptor-and chemokine-controlled adhesion and migration in chronic lymphocytic leukemia. Blood 119, 2590-2594.

la Fuente, de, M.A., Pizcueta, P., Nadal, M., Bosch, J., and Engel, P. (1997). CD84 leukocyte antigen is a new member of the Ig superfamily. Blood 90, 2398-2405. 


\section{Acknowledgements}

First, I would like to thank Henning Urlaub for his support, guidance and mentoring advice throughout this project. I thank Thomas Oellerich for providing endless samples, his scientific support and in general for the successful collaboration over the years. I would like to thank all the people from the Department of Internal Medicine, Hematology/Oncology at the University Hospital in Frankfurt am Main for providing samples, data, and input: Carmen Doebele, Silvia Münch, Roland Walter, Sebastian Mohr, and Hubert Serve. I would like to thank Kuan-Ting Pan for his contribution to this project, helpful scientific discussions and technical guidance. I thank Christof Lenz for having always on open ear for major and minor scientific problems and his contribution to this project. Moreover, I appreciate Christof Lenz and Kuan-Ting Pan for sharing their endless knowledge about mass spectrometry with me. I thank my thesis committee members Bernhard Küster and Reinhard Jahn for their time, their helpful input and advice. An additional 'Thank you' goes to Bernhard Küster as he had to travel from Munich to Göttingen for each meeting. Furthermore, I thank Simone Lemeer, Susan Kläger and Bernhard Küster for their contribution to this project with the kinobead assay. I thank Simone Lemeer for her support and advice in the early phase of this project. I would like to thank Debbie Yablonski from the 'Immune Cell Signaling Lab' at the Rappaport Faculty of Medicine, Technion, Israel Institute of Technology in Haifa, Israel and her group members Dvora Beach and Jennie Lugassy for providing samples, and helpful and interesting discussions. I thank Ekkehard Schütz, Julia Beck, Astrid Wachter, and Tim Beißbarth for their contribution to this project with RNA seq and the following data analysis. Especially, I would like to thank Astrid Wachter for her expertise and her bioinformatic consulting. I thank Hanibal Bohnenberger and Michael Engelke for their help with manual protein annotation. I thank Monika Raabe, Uwe Pleßmann, Annika Kühn, Lisa Neuenroth, and Irene Öchsner for their expert technical guidance and assistance. I thank Juliane Moses for organizational support. I thank all the people from the downstairs office for their friendship and support: Samir Karaca, Aleksandar Chernev, Alexandra Stützer, Christin Kappert, Kundan Sharma, and Sunit Mandad. I also thank the former lab members for their support in the early phase of this PhD project: Romina Hoefele, Katharina Kramer, Miroslav Nikolov, and He-Hsian Hsiao. I thank the Media Service, the IT department, and the administration of the MPIbpc for their excellent support. I would like to thank Magdalena Wienken for sharing her formatting skills and especially the friendship throughout the joint doctorate journey. I thank Marion Silies for her excellent proofreading 
skills. I thank my girls for their friendship, patience, and their understanding throughout all these years. I thank my family for their unconditional support that made all the achievements I made until now possible. Last but not least, I especially want to thank André Effe for accompanying me through the doctorate time while always being supportive, understanding and encouraging. 


\section{Appendices}

A Supplementary tables: All supplementary tables are stored on a CD that can be found attached at the back of the hard copy of this thesis.

Supplementary table S1: Time-resolved protein expression levels after BCR stimulation $(2,5$, 10, and $20 \mathrm{~min}$ ) of the Burkitt's lymphoma cell line DG75

Supplementary table S2: Time-resolved global phosphoproteome ( $\mathrm{SCX} / \mathrm{TiO}_{2}$ enrichment) dynamics after BCR stimulation (2, 5, 10, and $20 \mathrm{~min}$ ) of the Burkitt's lymphoma cell line DG75

Supplementary table S3: Time-resolved pYome (antibody-based phosphotyrosine enrichment) dynamics after BCR stimulation (2, 5, 10, and $20 \mathrm{~min}$ ) of the Burkitt's lymphoma cell line DG75

Supplementary table S4: Time-resolved BCR-dependent transcriptional changes, transcription factor activation and transcription factor $p$-site dynamics after BCR stimulation (10 min, 20 min, $1 \mathrm{~h}$, and $2 \mathrm{~h}$ ) of the Burkitt's lymphoma cell line DG75

Supplementary table S5: Time-resolved kinase affinity binding to kinobeads complemented with p-site mapping of time-resolved BCR stimulation $(2,5,10$, and $20 \mathrm{~min})$ of the Burkitt's lymphoma cell line DG75

Supplementary table S6: Protein expression values of the BRAF interactome analysis and $p$ sites of mapped BRAF interactors after BCR stimulation $(2,5,10$, and $20 \mathrm{~min})$ of the Burkitt's lymphoma cell line DG75

Supplementary table S7: Quantified and regulated $p$-sites after 5 min of BCR stimulation in combination with BRAF inhibition compared to 5 min of BCR stimulation of the Burkitt's lymphoma cell line DG75

Supplementary table S8: Quantified lysine acetylation and ubiquitination sites after 2 min of BCR stimulation of the Burkitt's lymphoma cell line DG75

Supplementary table S9: Commonly quantified and differential $p$-sites identified after timeresolved BCR stimulation in three different Burkitt's lymphoma cell models (DG75, Daudi and FFM_BL1) after pYome analysis (antibody-based phosphotyrosine enrichment)

Supplementary table S10: Quantified and differentially regulated p-sites in antigen-dependent and tonic BCR signaling identified from the Burkitt's lymphoma cell line DG75 after pYome analysis (antibody-based phosphotyrosine enrichment)

Supplementary table S11: Quantified and regulated $p$-sites identified from two AML cell lines, KG1 and MV4-11, after BTK inhibition from the pYome analysis (antibody-based phosphotyrosine enrichment)

Supplementary table S12: Regulated p-sites identified from two AML cell lines, KG1 and MV411 , after BTK inhibition from global phosphoproteomic analysis $\left(\mathrm{SCX} / \mathrm{TiO}_{2}\right.$ enrichment)

Supplementary table S13: Time-resolved $p$-site dynamics after TCR stimulation of the signaling adapters SLP76/LCP2 and GADS/GRAP2 


\section{B Mass spectrometric settings}

In the following detailed LC-MS/MS instrumental settings are listed for the instruments used for this work: LTQ Orbitrap Velos, Q Exactive, Q Exactive HF, and Orbitrap Fusion. The following abbreviations are used: BR: biological replicate; GP: global phosphoproteome analysis i.e. SCX fractionation in combination with $\mathrm{TiO}_{2}$ phosphopeptide enrichment; pYome: antibody-based phosphotyrosine enrichment; PE: protein expression analysis; LC: liquid chromatography; TopN: Maximum number of MS2 scans that can be performed after one intact peptide precursor scan; AGC: automated gain control; IT: injection time; Th: Thomson, unit for the mass-to-charge ratio; MS1: intact peptide precursor scan, MS2: product ion scan of selected precursor; NCE: normalized collision energy; CID: collision induced dissociation ; HCD: higher energy collisional dissociation; DG75, Daudi and FFM_BL1: Burkitt's lymphoma cell lines; KG1 and MV4-11: acute myeloid leukemia cell lines.

\section{B.1 LTQ Orbitrap Velos}

Table 13: LTQ Orbitrap Velos settings

\begin{tabular}{l|l|l} 
Project & DG75 BCR stimulation time course & TCR signaling adapters \\
Experiment & GP/pY & BR $1 / 2 / 3$ \\
\hline Spray voltage [kV] & 1.8 & 1.8 \\
LC method length [min] & 118 & 50 \\
LC gradient length [min] & 101 & 33 \\
TopN & 10 & 10 \\
MS1 Resolution & 30000 & 30000 \\
MS1 AGC target & $1.00 \mathrm{E}+06$ & $1.00 \mathrm{E}+06$ \\
MS1 Maximum IT [ms] & 500 & 500 \\
Intensity threshold & $1.00 \mathrm{E}+04$ & $1.00 \mathrm{E}+03$ \\
Fragmentation mode & $\mathrm{CID}$ & $\mathrm{CID}$ \\
Isolation window [Th] & 2 & 2 \\
NCE [\%] & 35 & 35 \\
MS2 AGC target & $5.00 \mathrm{E}+04$ & $1.00 \mathrm{E}+04$ \\
MS2 Maximum IT [ms] & 100 & 100 \\
Dynamic exlusion [s] & 60 & 60 \\
Charge state inclusion [z] & $2,3,4+$ & $2,3,4+$ \\
Linear gradient [\%B] & $4-34 \%$ & $4-34 \%$
\end{tabular}


Appendices

\section{B.2 Q Exactive}

Table 14: Q Exactive settings

\begin{tabular}{|c|c|c|c|c|c|c|c|}
\hline \multirow{2}{*}{$\begin{array}{l}\text { Project } \\
\text { Experiment }\end{array}$} & \multicolumn{2}{|c|}{ DG75 BCR stimulation time course } & \multicolumn{2}{|c|}{ DG75 BRAF inhibition } & \multirow{2}{*}{$\begin{array}{l}\text { DG75 BRAF } \\
\text { interactome } \\
\text { PE }\end{array}$} & \multicolumn{2}{|c|}{ AML KG1/MV4-11 BTK inhibition } \\
\hline & $\mathrm{pY}$ & PE & PE & GP & & $P E / G P$ & KG1/MV11 pY \\
\hline Spray voltage [kV] & 2.3 & 1.8 & 1.8 & 1.8 & 1.8 & 1.8 & 1.8 \\
\hline LC method length [min] & 90 & 90 & 60 & 90 & 60 & 90 & 120 \\
\hline LC gradient length [min] & 80 & 82 & 52 & 81 & 52 & 81 & 110 \\
\hline TopN & 15 & 15 & 15 & 12 & 15 & 12 & 12 \\
\hline MS1 Resolution & 70000 & 70000 & 70000 & 70000 & 70000 & 70000 & 70000 \\
\hline MS1 AGC target & $1.00 \mathrm{E}+06$ & $1.00 \mathrm{E}+06$ & $1.00 \mathrm{E}+06$ & $1.00 \mathrm{E}+06$ & $1.00 \mathrm{E}+06$ & $1.00 \mathrm{E}+06$ & $1.00 \mathrm{E}+06$ \\
\hline MS1 Maximum IT [ms] & $60 \mathrm{~ms}$ & $60 \mathrm{~ms}$ & $60 \mathrm{~ms}$ & $60 \mathrm{~ms}$ & $60 \mathrm{~ms}$ & $60 \mathrm{~ms}$ & $60 \mathrm{~ms}$ \\
\hline Intensity threshold & $4.00 \mathrm{E}+04$ & $5.00 \mathrm{E}+04$ & $4.80 \mathrm{E}+04$ & $2.40 \mathrm{E}+04$ & $4.80 \mathrm{E}+04$ & $2.00 \mathrm{E}+04$ & $2.00 \mathrm{E}+04$ \\
\hline Fragmentation mode & $\mathrm{HCD}$ & $\mathrm{HCD}$ & $\mathrm{HCD}$ & $\mathrm{HCD}$ & $\mathrm{HCD}$ & $\mathrm{HCD}$ & $\mathrm{HCD}$ \\
\hline Isolation window [Th] & 2 & 2 & 2 & 2 & 2 & 2 & 2 \\
\hline NCE [\%] & 28 & 28 & 28 & 28 & 28 & 28 & 28 \\
\hline MS2 Resolution & 17500 & 17500 & 17500 & 35000 & 17500 & 35000 & 17500 \\
\hline MS2 AGC target & $2.00 \mathrm{E}+05$ & $2.00 \mathrm{E}+05$ & $2.00 \mathrm{E}+05$ & $1.00 \mathrm{E}+05$ & $2.00 \mathrm{E}+05$ & $1.00 \mathrm{E}+05$ & $1.00 \mathrm{E}+05$ \\
\hline MS2 Maximum IT [ms] & $60 \mathrm{~ms}$ & $60 \mathrm{~ms}$ & $50 \mathrm{~ms}$ & $120 \mathrm{~ms}$ & $50 \mathrm{~ms}$ & $120 \mathrm{~ms}$ & $120 \mathrm{~ms}$ \\
\hline Underfill ratio [\%] & 1.2 & 1.5 & 1.2 & 2.4 & 1.2 & 2.4 & 2.4 \\
\hline Dynamic exlusion [s] & $15 s$ & $18 \mathrm{~s}$ & $20 \mathrm{~s}$ & $20 \mathrm{~s}$ & $20 \mathrm{~s}$ & 20 & 20 \\
\hline Charge state inclusion [z] & $2,3,4$ & $2,3,4$ & $2,3,4$ & $2,3,4,5,6,7$ & $2,3,4$ & $2,3,4,5,6,7$ & $2,3,4,5,6,7$ \\
\hline Peptide match & preferred & preferred & preferred & preferred & preferred & preferred & preferred \\
\hline Linear gradient [\%B] & $4-34 \%$ & $4-32 \%$ & $4-36 \%$ & $4-37 \%$ & $4-36 \%$ & $4-37 \%$ & $4-37 \%$ \\
\hline
\end{tabular}




\section{B.3 Q Exactive HF}

Table 15: Q Exactive HF settings

\begin{tabular}{|c|c|c|c|c|}
\hline Project & $\begin{array}{l}\text { DG75 BCR } \\
\text { kd/Syk } \\
\text { inhibition }\end{array}$ & $\begin{array}{l}\text { Daudi BCR } \\
\text { stimulation time } \\
\text { course }\end{array}$ & $\begin{array}{l}\text { FFM_BL1 BCR } \\
\text { stimulation time } \\
\text { course }\end{array}$ & $\begin{array}{l}\text { TCR signaling } \\
\text { adapters }\end{array}$ \\
\hline Experiment & $\mathrm{GP} / \mathrm{pY}$ & $\mathrm{pY}$ & $\mathrm{pY}$ & 4. BR \\
\hline Spray voltage [kV] & 2.2 & 2.2 & 2.5 & 2.3 \\
\hline LC method length [min] & 90 & 90 & 120 & 90 \\
\hline LC gradient length [min] & 74 & 74 & 103 & 74 \\
\hline TopN & 20 & 20 & 30 & 15 \\
\hline MS1 Resolution & 120000 & 120000 & $120 \mathrm{~K}$ & $120 \mathrm{~K}$ \\
\hline MS1 AGC target & $1.00 \mathrm{E}+06$ & $1.00 \mathrm{E}+06$ & $1.00 \mathrm{E}+06$ & $1.00 \mathrm{E}+06$ \\
\hline MS1 Maximum IT [ms] & 40 & 40 & 50 & 80 \\
\hline Intensity threshold & $6.30 E+04$ & $6.30 \mathrm{E}+04$ & $3.00 E+04$ & $1.90 \mathrm{E}+04$ \\
\hline Fragmentation mode & HCD & HCD & $\mathrm{HCD}$ & HCD \\
\hline Isolation window [Th] & 1.4 & 1.4 & 2 & 1.4 \\
\hline NCE [\%] & 28 & 28 & 28 & 28 \\
\hline MS2 Resolution & 30000 & 30000 & 30000 & 30000 \\
\hline MS2 AGC target & $1.00 \mathrm{E}+05$ & $1.00 \mathrm{E}+05$ & $1.00 \mathrm{E}+05$ & $1.00 \mathrm{E}+05$ \\
\hline MS2 Maximum IT [ms] & 64 & 64 & 50 & 50 \\
\hline Underfill ratio [\%] & 4 & 4 & 1.5 & \\
\hline Dynamic exlusion [s] & 30 & 30 & 30 & 30 \\
\hline Charge state inclusion [z] & $2,3,4,5,6$ & $2,3,4,5,6$ & $2,3,4,5,6$ & $2,3,4,5,4$ \\
\hline Peptide match & preferred & preferred & preferred & Preferred \\
\hline Linear gradient [\%B] & $1-40-60 \%$ & $1-40-60 \%$ & $2-40-60 \%$ & $2-40-60 \%$ \\
\hline
\end{tabular}

\section{B.4 Orbitrap Fusion}

Table 16: Orbitrap Fusion settings

\begin{tabular}{l|l} 
Project & DG75 BCR stimulation time course \\
Experiment & Antibody-based acetyl- and GG-lysine enrichment \\
\hline Spray voltage [kV] & 2.3 \\
LC method length [min] & $90 \mathrm{~min}$ \\
LC gradient length [min] & $74 \mathrm{~min}$ \\
TopN & Top speed \\
MS1 Resolution & $120 \mathrm{~K}$ \\
MS1 AGC target & 50000 \\
MS1 Maximum IT [ms] & 50 \\
Intensity threshold & 50000 \\
Fragmentation mode & HCD \\
Isolation window [Th] & 1.6 \\
NCE [\%] & 30 \\
MS2 Resolution & $30 \mathrm{~K}$ \\
MS2 AGC target & $1.00 \mathrm{E}+04$ \\
MS2 Maximum IT [ms] & 128 \\
Fragmentation mode & $\mathrm{HCD}$
\end{tabular}


Appendices

Underfill ratio

Dynamic exlusion

$20 \mathrm{~s}$

Charge state inclusion

$2,3,4,5$

Peptide match

Linear gradient [\%B]

$2-44 \%$ 


\section{List of abbreviations}

$A B C \quad$ Ammonium bicarbonate

Ac Acetylation

ACN Acetonitrile

AGC Automated gain control

AML Acute myeloid leukemia

BCR B-cell receptor

BL Burkitt's lymphoma

bp Base pair

BSA Bovine serum albumine

cDNA Complementary DNA

CID Collision induced dissociation

Ctrl Control

Da Dalton

DDA Data-dependent acquisition

DHB Dihydrobenzoic acid

DIA Data-indendent acquisition

DMEM Dulbecco's modified Eagle medium

DMSO Dimethylsulfoxide

DNA Deoxyribonucleic acid

DTT Dithiothreitol

DUB Deubiquitinase

ETD Electron transfer dissociation

EtOH Ethanol

FCS Fetal calf serum

FDR False discovery rate

FTMS Fourier transform mass

spectrometry

GP Global phosphoproteome

HCD Higher energy collisional ethanesulfonic acid

Reversed-phase high

RP-HPLC performance liquid chromatography

IAA lodoacetamide

ID Inner diameter or identifier

IP Immunoprecipitation

K Lysine

kd Knock-down

KDAC Lysine deacetylase

LC Liquid chromatography

M Molar

max. IT Maximum injection time

mRNA Messenger RNA

$\begin{array}{ll}\text { MS/MS } & \text { Tandem mass spectrometry } \\ \text { MS1 } & \text { Peptide precursor spectrum } \\ \text { MS2 or } & \text { Product ion spectrum of a } \\ \text { MS/MS } & \text { selected precursor } \\ \text { NP-40 } & \text { Nonidet P-40 } \\ \text { P } & \text { Phosphorylation } \\ \text { PBS } & \text { Phosphate buffered saline } \\ \text { PE } & \text { Protein expression } \\ \text { ppm } & \text { Parts per million } \\ \text { pS } & \text { Phosphorylated serine } \\ \text { PSP } & \text { PhosphoSitePlus, protein } \\ \text { pT } & \text { serine/threonine phosphatase } \\ \text { PTM } & \text { Phosphorylated threonine } \\ \text { PTP } & \text { Post-translational modification } \\ \text { pY } & \text { Protein tyrosine phosphatase } \\ \text { R } & \text { Phosphorylated tyrosine } \\ \text { RNA } & \text { Arginine } \\ \text { RNAseq } & \text { Ribonucleic acid } \\ \text { rpm } & \text { Ribonucleic acid sequencing } \\ \text { RT } & \text { Rounds per minute } \\ \text { SCX } & \text { Room temperature } \\ & \text { Strong cation exchange } \\ \text { SDS-PAGE } & \text { Sodium dodecyl sulfate } \\ & \text { polyacrylamide gel } \\ \text { shRNA } & \text { Small hairpin ribonucleic acid } \\ \text { siRNA } & \text { Small interfering ribonucleic acid } \\ \text { SILAC } & \begin{array}{l}\text { Stable isotope labeling by amino } \\ \text { Ycids in cell culture }\end{array} \\ \text { StageTips } & \text { STop And Go Extraction Tips } \\ \text { TCR } & \text { T-cell receptor } \\ \text { TFA } & \text { Trifluoroacetic acid } \\ \text { TiO } & \text { Titanium dioxide } \\ \text { Tris } & \text { Trisamine } \\ \text { U } & \text { Unit } \\ \text { Ub } & \text { Ubiquitination } \\ \text { WB } & \text { Western Blot } \\ \text { WT } & \text { Wild type } \\ \text { Times g-force } \\ \text { Tyrosine } \\ \text { Pg }\end{array}$

